\title{
POLTTÉCNICA
}

UNIVERSIDAD POLITÉCNICA DE MADRID

E.T.S.I. AGRONÓMICA, ALIMENTARIA Y DE BIOSISTEMAS

\section{Estudio Geométrico del Caos en Sistemas Hamiltonianos con dos o más grados de Libertad.}

\section{Tesis Doctoral}

\section{Alberto Vergel Otero}

Licenciado en Ciencias Matemáticas

Madrid, 2020 



\title{
POLITÉCNICA
}

UNIVERSIDAD POLITÉCNICA DE MADRID

E.T.S.I. AGRONÓMICA, ALIMENTARIA Y DE BIOSISTEMAS DEPARTAMENTO DE INGENIERÍA AGROFORESTAL,

\section{Estudio Geométrico del Caos en Sistemas Hamiltonianos con dos o más grados de libertad.}

\author{
Tesis Doctoral \\ Alberto Vergel Otero \\ Licenciado en Ciencias Matemáticas
}

Directores

Rosa María Benito Zafrilla Juan Carlos Losada González

Doctora en Ciencias Químicas Doctor en Ciencias Físicas

Madrid, 2020 

A mi familia. 

' No intentes cambiar un sistema, construye uno nuevo que haga que el anterior se vuelva obsoleto'

R. Buckminster 'BuCKY' Fuller (1895-1983) 



\section{Agradecimientos}

Son muchas cosas, las circunstancias, las personas más próximas y los proyectos más lejanos que otros construyeron lo que nos empuja a crecer, citarlos a todos sería poco menos que imposible.

Lo primero, dar gracias por las condiciones y circunstancias que me han permitido dedicar tiempo a este trabajo, sin ellas mi esfuerzo hubiera sido en vano.

Dar gracias por los errores, que me han enseñado que disfrazados de fracasos escondían grandes oportunidades y me han obligado a cambiar, marcando así las líneas generales de este trabajo.

Por otro lado decir que sólamentente nos construimos desde dentro y a través de los demás, crecemos empujados por aquellas personas que de alguna manera llevamos dentro de nuestro corazón, mi agradecimiento a estas personas.

A mi familia, por todo el apoyo y el amor demostrado durante todo este tiempo de trabajo.

A mis directores de tesis, Rosa y Juan Carlos por el tiempo que hemos compartido juntos en el departamento, por dejarme desarrollar el trabajo que realmente me apetecía y por darme la llave del departamento al tercer día de conocernos en un ejercicio de confianza que me dejó claro que quería trabajar con ellos.

A Tino por las conversaciones en el ICMAT y por sus comentarios que siempre he tenido en consideración.

A Pedro por las escasas pero fructíferas charlas de matemáticos que mantuvimos en el departamento.

A los que se ilusionan.

A la vida.

A.M. 



\section{Resumen}

En esta tesis hemos implementado el formalismo geometrodinámico con el fin de estudiar desde esta perspectiva la estructura del espacio de fases de diversos sistemas dinámicos hamiltonianos realistas. En particular aplicamos dicho formalismo al sistema de isomerización LiNC-LiCN bidimensional y al sistema Hénon-Heiles, también hemos encontrado un criterio novedoso de suficiencia para la integrabilidad de sistemas dinámicos tridimensionales.

Siguiendo la metodología geometrodinámica hemos definido una variedad riemanniana (variedad mecánica) dotada con la métrica de Jacobi integrando numéricamente las ecuaciones geodésicas para obtener las trayectorias clásicas.

En dos grados de libertad el sistema de isomerización LiNC-LiCN está configurado por un enlace triple $\mathrm{C} \equiv \mathrm{N}$, por lo que en nuestro modelo mantenemos constante la distancia entre el $\mathrm{C}$ y el $\mathrm{N}$ en su valor de equilibrio. La molécula tiene dos isómeros estables correspondiéndose con los dos mínimos de la función de energía potencial realista obtenida por métodos ab initio que son dos puntos elípticos, separados por una barrera energética relativamente baja.

Para estudiar la sensibilidad a condiciones iniciales del sistema, analizaremos la evolución del vector separación geodésica definida por la ecuación de Jacobi-Levi-Civita integrándola numéricamente a lo largo de trayectorias geodésicas para diferentes condiciones iniciales, permitiendo así estudiar la estructura del espacio de fases de este sistema.

Observamos que las trayectorias en su recorrido siempre se acercan a la frontera de la región accesible de su espacio de configuraciones (región de Hill). Utilizando la misma metodología, estudiamos la influencia que la curvatura de esta frontera alrededor del punto de retorno tiene en la naturaleza regular o caótica de la trayectoria en cuestión.

Comparamos nuestro sistema dinámico con el problema de billar clásico y comprobamos el papel de la curvatura escalar en el interior de la región de Hill para estabilizar la trayectoria independientemente de la curvatura del punto de retorno.

Apoyándonos en la posibilidad de plantear el problema del arco catenario y su estabilidad desde la formulación geometrodinámica extendemos esta idea a sistemas dinámicos identificando cuerdas flexibles con trajectorias del sistema. Proponemos un novedoso indicador de caos válido para sistemas de dos y tres 
dimensiones, aplicándolo al sistema dinámico Hénon-Heiles y encontrando que la trayectoria más estable cumple lo que denominamos condición catenaria generando regiones de estabilidad local.

Midiendo cuan alejada se encuentra la trayectoria de estudio de ser trayectoria catenaria definimos un indicador de caos para una trayectoria cualquiera en sistemas dinámicos de dos y tres dimensiones.

Observamos que la regularidad de estas curvas catenarias es consecuencia de la existencia de una simetría local o campo Killing a lo largo de ellas.

Finalmente extendiendo esta idea a sistemas de más de dos grados de libertad, proponemos un criterio suficiente de integrabilidad para sistemas hamiltonianos tridimensionales. Dicho criterio afirma que en aquellos sistemas con matriz de masa unidad en los que exista una foliación del espacio de configuraciones por superficies formadas por curvas catenarias podremos asegurar la integrabilidad del sistema.

Aplicamos este criterio a diferentes sistemas dinámicos tridimensionales. 


\section{Abstract}

In this work we have worked with the geometrodynamic formalism estudying from this point of view the phase space structure for different realistic Hamiltonian systems. With this method we have analized the two-dimension isomerization system LiNC-LiCN as well as the Hénon-Heiles system, finding as well a new sufficiency criterion for integrability applied to three-dimension dynamical systems.

From this point of view we have defined a Riemmanian manifold (mechanical manifold) dowed with the Jacobi metric integrating numerically the geodesic equations resulting the classical trajectories.

In the two-dimension isomerization model LiNC-LiCN, because of the triple bond $\mathrm{C} \equiv \mathrm{N}$, we consider the distance between the two atoms $\mathrm{C}$ and $\mathrm{N}$ frozen at the equilibrium value. The realistic potential energy function is calculated by ab initio method, two stable isomers or elliptic points corresponding to the minima are determined separated by a low energetic barrier.

In order to study the sensibility to initial conditions, we analyze the evolution for the geodesic separation vector field controlled by the Jacobi-Levi-Civita equation, integrating numerically this equations with the geodesics for different initial conditions and analyzing the phase space structure of the system.

We observe that trajectories allways bounce the accesible region boundary (Hill boundary). We study with the same method the influence of the curvature of the boundary at the turning points for the regularity or chaoticity of the trajectory.

Analizing the analogy with the classical billard and studying the influence of the scalar curvature inside the Hill region as stabilization mechanism for trajectories independently of the curvature at the turning points.

Supported with the possibility of studying the catenary arch and its stability from the geometrodynamic formulation, we extends this idea to dynamical systems identifying flexible strings with trajectories of the dynamical system. We propose a new indicator of chaos for two and three-dimension systems, studying the Hénon-Heiles system showing that the most stable trajectories fulfilling the catenary condition generate local stability regions.

Measuring how far the trajectory separates from a catenary we define an chaos indicator for trajectories in two and three-dimension dynamical systems. 
Regularity of these trajectories is deduced from the existence of a local symmetry or Killing vector field along the curve.

Finally, we propose to extend this idea to dynamical systems with more than two degrees of freedom, proposing a new sufficiency criterion of integrability for three-dimension Hamiltonian systems. The criterion stays that those systems with unit mass matrix where surfaces generated by catenaries curves are folliating the whole accesible space are integrable.

We apply this criterium to different three-dimension dynamical systems. 


\section{Índice general}

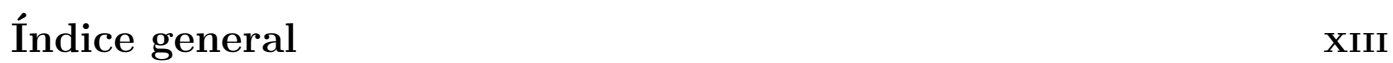

\begin{tabular}{ll}
\hline 1. Introducción & 1
\end{tabular}

\begin{tabular}{lr}
\hline 2. Formulación clásica & $\mathbf{7}$
\end{tabular}

2.1. Formulación lagrangiana. Ecuaciones de Euler-Lagrange. . . . . 7

2.2. Formulación hamiltoniana . . . . . . . . . . . . . . . . . 9

2.3. Formulación simpléctica . . . . . . . . . . . . . . . . . 11

2.4. Transformaciones canónicas. Función

generatriz . . . . . . . . . . . . . 16

2.5. Ecuación de Hamilton-Jacobi . . . . . . . . . . . . . . . . 18

2.6. Variables acción-ángulo . . . . . . . . . . . . . . . . . . . . . . . 19

2.6.1. Movimiento multidimensional . . . . . . . . . . . 20

\begin{tabular}{|lr}
\hline 3. Formalismo geometrodinámico & 23
\end{tabular}

3.1. Proceso de geometrización y Métrica de Jacobi. . . . . . . . . . 23

3.1.1. Ecuaciones geodésicas con parámetro físico (t) . . . . . 25

3.2. Métrica Jacobi y óptica geométrica . . . . . . . . . . . . . . . . 27

3.3. Métrica Jacobi y Principio de Maupertuis . . . . . . . . . . . . 28

3.4. La métrica Eisenhart . . . . . . . . . . . . . . . . . . . . . . . . 29

3.5. Ejemplos . . . . . . . . . . . . . . . . . . . . . . . 32

3.5.1. Ejemplo 1 . . . . . . . . . . . . . . . . 33

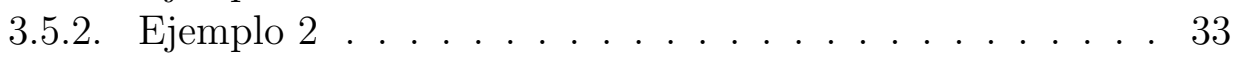

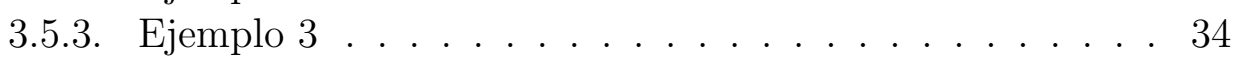

3.5.4. Versión geometrodinámica . . . . . . . . . . . . . . 35

\begin{tabular}{ll}
\hline 4. Estabilidad y Caos & 37
\end{tabular}

4.1. Indicadores clásicos . . . . . . . . . . . . . . . . . . . . . . . . . 38

4.1.1. Mapa y Sección de Poincaré . . . . . . . . . . . . . . . . 39

4.1.2. Exponente de Lyapunov . . . . . . . . . . . . . . . . . . 40

4.1.3. $\quad$ Espectro de frecuencias. Transformada de Fourier . . . . 44

4.2. Ecuación JLC e indicador SGI . . . . . . . . . . . . . . . . . . . 45

4.2.1. Dimensión dos: . . . . . . . . . . . . . . . . . . . . . . 48 
4.2.2. $\quad$ Parametrización por tiempo físico $t$. . . . . . . . 52

4.2.3. Dimensión tres . . . . . . . . . . . . . . . . 53

4.3. Puntos conjugados. . . . . . . . . . . . . . . 54

4.3.1. Puntos conjugados . . . . . . . . . . . . . . 55

$4.3 .2 . \quad$ Ejemplos . . . . . . . . . . . . . . . . 56

5. Modelo de isomerización LiNC-LiCN 65

5.1. Descripción del modelo . . . . . . . . . . . . . . . . . . 65

5.2. Formulación geometrodinámica del modelo LiNC-LiCN . . . . . 68

5.2.1. Las ecuaciones JLC . . . . . . . . . . . . . . . . . . . 69

5.3. Aplicación del indicador SGI al modelo LiNC- LiCN . . . . . . . 70

5.3.1. Trayectorias clásicas para LiNC-LiCN]. . . . . . . . . . 70

5.3.2. Método geométrico aplicado a trayectorias LiNC y LiCN 71

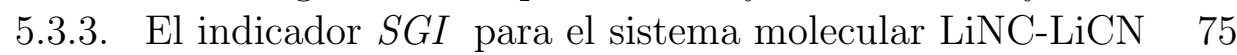

5.3.4. Conclusiones de la sección . . . . . . . . . . . 76

5.4. Convexidad de la frontera de la región de Hill e inestabilidad . . 77

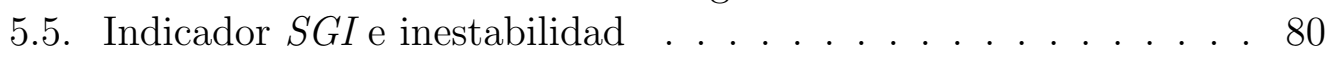

5.5.1. Conclusiones de la sección . . . . . . . . . . . . . 83

6. Condición catenaria: indicador de caos 85

6.1. Fundamento teórico . . . . . . . . . . . . . . 85

6.2. Principio de Trabajos Virtuales

Geometrodinámico .................. 88

6.3. La curva catenaria: paradigma de

estabilidad . . . . . . . . . . . . . . 88

6.3.1. La hipótesis . . . . . . . . . . . . . . . . . . . . . 91

6.4. Geometrodinámica y generalización del

Teorema de Trabajos Virtuales . . . . . . . . . . . . . 92

6.5. Aplicación al sistema Hénon-Heiles 2D . . . . . . . . . . . . . . 94

6.5.1. GVWP aplicado al sistema Hénon-Heiles 2D . . . . . . . 95

6.6. Definiendo el indicador de caos . . . . . . . . . . . . . . . . 96

6.6.1. Análisis de trayectorias . . . . . . . . . . . . . . 98

6.7. Resultados numéricos . . . . . . . . . . . . . . . . . . . 98

\begin{tabular}{ll}
\hline 7. Simetría e integrabilidad & 101
\end{tabular}

7.1. Simetría: vectores y campos Killing (KVF) . . . . . . . . . . . . 102

7.1.1. Problema de Kepler 2D en coordenadas polares . . . . . 104

7.1.2. Problema de Kepler 2D en coordenadas cartesianas . . . 105

7.2. Campos tensoriales Killing . . . . . . . . . . . . . . 107

7.2.1. Buscando campos vectoriales Killing . . . . . . . . . . . 108

7.3. Condiciones de integrabilidad para sistemas 3D . . . . . . . . . 112

7.3.1. Condición de integrabilidad . . . . . . . . . . . . . 114

7.4. Aplicaciones . . . . . . . . . . . . . . . . 115 


\section{APÉNDICES}

A.1. Métrica de Jacobi y regla de cuantización de Einstein . . . . . . 137 A.1.1. Cuantización del momento angular . . . . . . . . . . 138

A.2. Aplicación: el átomo hidrogenoide . . . . . . . . . . . . . . . . 138 A.2.1. Estudio de estabilidad. Estado fundamental . . . . . . . 141 A.3. Cuantización topológica: aplicación al oscilador armónico . . . . 142

A.4. Ecuación de Schrödinger y métrica Jacobil. . . . . . . . . . . . . . . . . . . . . . . . . . . . 143

B. Geometría diferencial. Tensores

B.1. Álgebra vectorial . . . . . . . . . . . . . . . . . . . 152

C. Principio de mínima curvatura de Hertz 


\section{Capítulo 1}

\section{Introducción}

Una gran mayoría de problemas en Física implican ecuaciones no lineales, lo que supone la existencia de trayectorias caóticas donde la predicción de la evolución futura del sistema es imposible debido a la sensibilidad a condiciones iniciales, esto es, dos trayectorias inicialmente próximas acaban separándose exponencialmente en el tiempo, característica principal de la dinámica caótica.

En nuestro trabajo estudiaremos el denominado caos determinista surgido de ciertos sistemas dinámicos conservativos, donde las trayectorias serán soluciones de ecuaciones de movimiento deterministas (Ecuaciones de Hamilton) y la energía es una constante de movimiento.

Utilizaremos para ello un enfoque geometrodinámico, es decir, reformularemos el problema dinámico en términos geométricos describiendo el espaciotiempo y los fenómenos físicos en términos de geometría diferencial; así, por ejemplo, las trayectorias dinámicas se identificarán con geodésicas de una cierta variedad Riemanniana y el tiempo será interpretado como el parámetro longitud de arco de estas curvas.

Definiremos un indicador de caos novedoso con el que analizaremos un sistema hamiltoniano realista de tipo molecular, el modelo de Isomerización LiNC-LiCN de dos grados de libertad, estudiando su comportamiento en régimen de regularidad y encontrando el umbral de energía para la aparición de regiones caóticas bien definidas estudiando posteriormente su estructura.

Igualmente y tomando como modelo de estabilidad la curva catenaria, generalizaremos el Principio de trabajos virtuales definiendo a partir aquí un nuevo indicador de caos con el que analizamos otros sistemas dinámicos de interés como el modelo Hénon-Heiles desde la metodología geometrodinámica, encontrando finalmente una novedosa condición de suficiencia para la integrabilidad de sistemas dinámicos hamiltonianos tridimensionales con tensor de 
masa identidad.

Nuestro objetivo será aportar nuevas herramientas para profundizar en el estudio de las zonas de regularidad y caos de estos sistemas dinámicos e incluso para la determinación de su posible integrabilidad siempre utilizando el enfoque geometrodinámico, identificando el origen geométrico de la inestabilidad de ciertas trayectorias y del posible caos.

A continuación detallamos las razones por las que esta reformulación geométrica del espaciotiempo y sus procesos es tan fructífera, y cómo se fue forjando a base de superar las diferentes dificultades que surgían de las teorías físicas de cada momento, siempre con la idea de generalizar un marco teórico en el que el hecho físico sea el protagonista y su descripción no dependa del observador, las predicciones se ajusten a los resultados experimentales y la escala no sea una limitación de la teoría.

Identificar elementos de la Naturaleza con objetos geométricos, está en el corazón por el afán de hacer del Universo un lugar comprensible.

En esta línea si hay un hecho que yo destacaría como origen de esta revolución intelectual es la invención/descubrimiento de la geometría axiomática griega, trasladándola del mundo de la construcción al mundo de la lógica y como consecuencia empujando a la Naturaleza al mundo de lo inteligible.

Platón es el primero que hace una geometrización sistemática de la Naturaleza asociando a cada elemento básico de ésta un poliedro regular, un sólido platónico. Pero incluso más allá de este logro, construye la primera teoría de la cuantización asociando a cada elemento básico natural un número discreto, bien sea de caras, aristas o vértices del poliedro correspondiente.

Pero Platón no puede hacer una geometrodinámica en cuanto su geometría tiene únicamente carácter descriptivo, estático, no dinámico. La predicción a partir de esta representación geométrica de los sucesos físicos no es posible.

Avancemos en el tiempo con la idea de geometrizar el mundo...

Cualquiera que haya leído los Philosophiae Naturalis Principia mathematica de Sir Isaac Newton Newton, 1686 se ha encontrado con un texto que, excepto en las definiciones iniciales y los pocos axiomas posteriores, está escrito estrictamente en términos geométricos. Este método se muestra aquí como una vía para descubrir el mundo cambiante a escalas muy diversas, con una capacidad predictiva sin precedentes.

A pesar de esto, para la física newtoniana la geometría del espacio es euclídea y el hecho físico se desarrolla por así decirlo superpuesto sobre esta estructura geométrica siendo ambas independientes, sin comunicarse y sin alterarse mutuamente. Por otro lado el tiempo, que juega un papel de parámetro en la teoría y transcurre de igual forma para todo observador, podríamos decir que es único y universal.

Si bien la geometrización realizada hasta ese momento había obtenido resultados incomparables con ninguna otra teoría anterior, aún había ciertas 
cuestiones abiertas:

En el estudio de las órbitas planetarias en el Sistema Solar la teoría newtoniana funciona perfectamente excepto cuando se estudian órbitas próximas al Sol, como la de Mercurio donde la precesión de su perihelio no puede ser descrita y mucho menos comprendida utilizando la física newtoniana.

Además, las ecuaciones de Newton son simétricas respecto del tiempo y por tanto todo suceso mecánico sería en principio reversible pero la termodinámica vendrá a contradecir esta afirmación.

Igualmente no podía dar respuesta a los fenómenos a escala atómica que ya a principios de siglo habían sido propuestos por M. Plack en 1900 y al efecto fotoeléctrico que describió Einstein en 1905 [Einstein, 1905].

Desde el punto de vista teórico la física newtoniana planteaba dos dificultades atendiendo a dos principios fundamentales:

Principio de relatividad de carácter fundamental al afirmar que si tomamos distintos sistemas de referencia admisibles, todos los fenómenos físicos transcurren del mismo modo, por lo que no podemos determinar en cuál de estos sistemas nos encontramos.

Principio de covarianza donde las leyes de la Física deben tomar la misma forma en todos sistemas de referencia.

En el caso de las ecuaciones de Newton, el Principio de relatividad solamente acepta como sistemas de referencia admisibles los inerciales y las transformaciones de Galileo. En cuanto el movimiento del sistema de referencia es acelerado, por ejemplo en el movimiento circular, aparecen términos nuevos en las ecuaciones y, por tanto, su expresión deja de ser invariante, contradiciendo el Principio de covarianza.

Las transformaciones de Galileo dejan forminvariante la métrica euclidea por lo que deducimos que el espacio es euclideo, homogéneo e isótropo, pero esta limitación de sistemas admisibles ponía en tela de juicio la validez general de las ecuaciones de Newton.

En 1873 J. C. Maxwell [Maxwell, 1873], a partir de los trabajos de A. Ampére, M. Faraday y H. Lenz describe las leyes del campo electromagnético, un sistema de ecuaciones de origen experimental que, a diferencia de las leyes de Newton, no eran invariantes respecto a las transformaciones de Galileo.

Más adelante, en 1881, Michelson Michelson, 1881 muestra que la velocidad de un haz luminoso detectado por dos observadores diferentes en movimientos relativos perpendiculares es la misma, tirando por tierra el Principio de relatividad de Galileo e insinuando el carácter invariante de la velocidad luz.

Curiosamente son las Transformaciones de Lorentz las que dejan forminvariante las ecuaciones de Maxwell, siendo éstas transformaciones lineales en las que el tiempo aparece como una variable nueva dejando de ser simplemente un parámetro además la velocidad de la luz aparece como una constante universal. 
A la vez, y durante todo el siglo XIX, N. Lobachevsky (Rusia) en 1829 Lobachevski, 1829, J. Bolyai (Rumanía) en 1832 Bolyai, 1832, C.F. Gauss (Alemania) Gauss, 1843] , B. Riemann (Alemania) 1854 [Riemann], E. Beltrami (Italia) en 1868 [Beltrami] y F. Klein (Alemania) en 1872 Klein, 1872 plantean la posibilidad de una nueva geometría en la que no todos los postulados de Euclides se cumplan, en particular el quinto. Como resultado de este esfuerzo colectivo en 1908 H. Minkowki (Alemania) Minkowki, 1908 propone concebir el mundo como un continuo espacio-tiempo.

Esta idea influyó mucho en A. Einstein que, junto con el concepto de variedad riemanniana y el Principio de equivalencia, dio como resultado la Teoría de la Relatividad General (TRG) en 1915 Einstein, 1905.

Desde entonces el mundo se podrá pensar como una variedad riemanniana cuatri-dimensional caracterizada por un tensor métrico (producto escalar) pseudo-riemanniano (no definido positivo) y determinando en cada punto una curvatura relacionada directamente con la densidad de energía acumulada en ese punto.

En este nuevo enfoque la energía en todas sus formas y la materia quedan unificadas en una nueva entidad geométrica denominada tensor de energíamomento que informa a la geometría acerca de cómo curvarse y ésta última le dice al sistema cómo evolucionar en el espacio y en el tiempo, mostrándose la unidad entre masa-energía y geometría del espacio-tiempo.

Este nuevo enfoque se ha mostrado muy fructífero y sus predicciones se ajustan perfectamente a los datos experimentales en relación por ejemplo al problema citado del perihelio de Mercurio, las lentes gravitatorias, la existencia de agujeros negros y la física de las estrellas de neutrones.

Además esta geometrodinámica relativista ha demostrado la naturaleza geométrica de la acción gravitatoria desechando la acción a distancia presente en toda la teoría gravitatoria Newtoniana.

En nuestro trabajo haremos un tipo especial de geometrodinámica basada en el principio que P. L. Maupertuis expuso entre 1740 y 1748 [Maupertuis, 1940-, afirmando que en todos los fenómenos naturales una cierta cantidad llamada acción tiende a ser minimizada, en su caso el producto de la masa por la velocidad y la distancia recorrida.

Esta idea, que está en los fundamentos del Cálculo Variacional y, en particular, del Principio de Acción Estacionaria junto con los desarrollos de L. Euler, J. L. Lagrange y W. R. Hamilton, nosotros la reformularemos en términos geométricos reescribiendo el funcional de acción de Maupertuis como la diferencial de longitud en una variedad Riemanniana dotada con cierta métrica denominada métrica de Jacobi.

En esta variedad riemanniana también llamada variedad mecánica la métrica será definida positiva (a diferencia de la semidefinida en la TRG) pero identificaremos igualmente magnitudes dinámicas y geométricas como hemos 
citado previamente.

Esta geometrización de la dinámica a través de la métrica de Jacobi, desarrollada en los capítulos 2 y 3, nos permite describir la evolución de un sistema clásico hamiltoniano con tensor de masa no dependiente de las velocidades a través del Principio de Hertz de mínima curvatura donde una partícula no sometida a fuerzas exteriores se moverá a lo largo de trayectorias de mínima curvatura.

Con la métrica de Jacobi podemos reducir la dinámica a cinemática, donde la fuente de la dinámica, en nuestro caso la energía potencial, se incorpora a la propia métrica del espacio curvando el espacio vacío.

En el capítulo 4 aplicaremos este formalismo para estudiar las regiones de estabilidad del espacio de fases del sistema a través de la construcción de varios indicadores de caos basados principalmente en la ecuación de Jacobi-LeviCivita, indicadores de la sensibilidad a condiciones iniciales de las trayectorias.

Los indicadores de caos más utilizados en la literatura Fast Lyapunov Indicator (FLI), Mean Exponential Growth factor of Nearby Orbits (MEGNO), Generalized Alignment Indice (GALI), Smaller Alignment Indice (SALI), exponentes Lyapunov, análisis del mapa de frecuencias, Secciones de Poincaré para dos grados de libertad, etc.. están definidos en el espacio de fases. Por el contrario, nuestro caso nuestro indicador se define sobre los flujos geodésicos definidos en el espacio de configuración.

En el capítulo 5 aplicaremos la teoría geometrodinámica al estudio de sistemas moleculares realistas, en particular al sistema de isomerización LiNC-LiCN en dos dimensiones, y definiremos de manera novedosa un indicador de caos con el que estudiaremos principalmente las regiones de estabilidad y caos de este sistema demostrando que la resonancia paramétrica es el origen geométrico del caos para este sistema.

Igualmente, demostraremos cómo este sistema no es reducible a un problema de billar clásico, descubriendo que la convexidad de la frontera de la región accesible al sistema denominada región de Hill influye pero no determina la naturaleza regular o caótica de las trayectorias del sistema, desvelando de manera novedosa el papel tan importante de la curvatura escalar para la estabilidad de la trayectoria.

En el capítulo 6 utilizando el formalismo anterior a través de la métrica de Jacobi construimos un indicador de estabilidad y caos igualmente novedoso basándonos únicamente en criterios de estática fijándonos en la estabilidad del árco catenario y la conocida proposición de David Gregory extraída del ámbito de la mecánica de estructuras. Aplicaremos dicho indicador al modelo de Hénon-Heiles en dos dimensiones para estudiar las regiones de estabilidad a diferentes energías.

En el capítulo 7, a partir de los desarrollos mostrados en el capítulo 6, utilizaremos el concepto de simetrías Killing con sus cantidades conservadas 
asociadas para, generalizando este concepto definir un criterio novedoso de suficiencia para la determinación de la Integrabilidad de un sistema dinámico en tres dimensiones.

En el capítulo 8 enumeramos las conclusiones obtenidas en esta tesis y a continuación se presenta la bibliografía.

Por último incluimos unos Apéndices. En el primero mostramos la validez de la aplicación de la métrica de Jacobi para la cuantización de un sistema dinámico observando que la regla de cuantización de Einstein no es más que un cálculo básico de la longitud de la trayectoria en esta métrica obteniendo la ecuación de Schrödinger directamente de esta aplicación y aplicándo el formalismo al estudio de la cuantización del átomo de Hidrógeno. Igualmente el formalismo geometrodinámico se muestra efectivo a la hora de establecer la cuantización topológica aplicando el Teorema de Gauss-Bonnet aplicándolo a la cuantización del oscilador armónico.

En los siguientes anexox se desarrollan los conceptos geométricos básicos para seguir el desarrollo matemático: cálculo tensorial como lenguaje básico en el que expresar las ecuaciones de manera forminvariante (Fok) y principio de mínima curvatura de Hertz, conceptos básicos para la comprensión del trabajo. 


\section{Capítulo 2}

\section{Formulación clásica}

Para el estudio de la dinámica de un sistema físico se ha mostrado exitoso el planteamiento variacional, al poder encontrar sus ecuaciones de evolución independientemente del sistema de coordenadas utilizado.

Este enfoque se basa en el uso del Principio de acción estacionaria que aplicado a una determinada integral de acción permite deducir las ecuaciones de evolución del sistema en el tiempo.

Este método se aplica en muchas parcelas de la Física bien sea clásica, cuántica o relativista; cada una de ellas se puede estudiar a partir de una determinada acción y la aplicación de este principio a su integral de acción.

\subsection{Formulación lagrangiana. Ecuaciones de Euler-Lagrange}

Sea la función lagrangiana

$$
L=L(\mathbf{q}(t), \dot{\mathbf{q}}(t), t) \quad \text { con } \quad \dot{\mathbf{q}}(t)=\frac{d \mathbf{q}}{d t},
$$

definida en el espacio de configuración $(\mathbf{q}(t), \dot{\mathbf{q}}(t))$. Si construimos la integral de la función lagrangiana $L$ a lo largo de la curva $\gamma$ de representación paramétrica $\mathbf{q}(t)$ y vector tangente $\dot{\mathbf{q}}(t)$ como

$$
S[\gamma]=\int_{t_{1}}^{t_{2}} L(\mathbf{q}(t), \dot{\mathbf{q}}(t), t) d t
$$

podemos encontrar las trayectorias solución al problema dinámico aplicando el Principio de acción estacionaria sobre la integral de acción $S$, obteniendo 
las siguientes ecuaciones diferenciales de segundo orden (ecuaciones de EulerLagrange):

$$
E_{l}(L)=\frac{d}{d t}\left(\frac{\partial L}{\partial \dot{q}^{l}}\right)-\frac{\partial L}{\partial q^{l}}=0,
$$

donde $E_{l}(L)=$ es la l-ésima componente del vector de Euler-Lagrange de L y es un covector, esto es, un tensor covariante de orden 1 que representa la generalización del gradiente de $L$. Por ser (2.3) igualdad tensorial ésta no dependerá del sistema coordenado utilizado (Fok).

De aquí en adelante utilizaremos notación Einstein $a^{i} b_{i}=a^{1} b_{1}+\cdots+a^{n} b_{n}$ además de indistintamente

$$
\frac{\partial f}{\partial q^{l}}=\partial_{l} f \quad, \quad \frac{d q^{i}}{d t}=\dot{q}^{i}, a_{i j}(\mathbf{q})=a_{i j} .
$$

Si la función lagrangiana tiene la forma

$$
L(\mathbf{q}, \dot{\mathbf{q}}, t)=\frac{1}{2} a_{i j}(\mathbf{q}) \frac{d q^{i}}{d t} \frac{d q^{j}}{d t}-V(\mathbf{q})=\underbrace{T\left(q^{i}, \frac{d q^{i}}{d t}\right)}_{\text {E.Cinética }}-\underbrace{V\left(q^{i}\right)}_{\text {E.Potencial }}
$$

entonces

$$
\begin{gathered}
\frac{\partial L}{\partial q^{l}}=-\frac{\partial V}{\partial q^{l}}+\frac{1}{2} \frac{\partial a_{i j}}{\partial q^{l}} \dot{q}^{i} \dot{q}^{j} \\
\frac{\partial L}{\partial \dot{q}^{l}}=a_{l k} \dot{q}^{k} \\
E_{l}(L)=\frac{d}{d t}\left(\frac{\partial L}{\partial \dot{q}^{l}}\right)-\frac{\partial L}{\partial q^{l}}=\partial_{j} a_{l k} \dot{q}^{j} \dot{q}^{k}+a_{l k} \ddot{q}^{k}-\frac{1}{2} \partial_{l} a_{i j} \dot{q}^{i} \dot{q}^{j}+\partial_{l} V=0
\end{gathered}
$$

expresamos esta igualdad tensorial en forma contravariante multiplicando por $a^{i l}$, esto es, aplicamos la operación de subir índices, así,

$$
\begin{array}{r}
a^{i l} \partial_{j} a_{l k} \dot{q}^{j} \dot{q}^{k}+\underbrace{a^{i l} a_{l k}}_{\delta_{k}^{i}} \ddot{q}^{k}-\frac{1}{2} a^{i l} \partial_{l} a_{i j} \dot{q}^{i} \dot{q}^{j}+a^{i l} \partial_{l} V=0 \Rightarrow \\
\ddot{q}^{i}+\frac{1}{2} a^{i l}\left[\partial_{j} a_{l k} \dot{q}^{j} \dot{q}^{k}+\partial_{j} a_{l k} \dot{q}^{j} \dot{q}^{k}-\partial_{l} a_{i j} \dot{q}^{i} \dot{q}^{j}\right]+a^{i l} \partial_{l} V=0 \Rightarrow
\end{array}
$$

haciendo el cambio de índices $i \longleftrightarrow j$ y utilizando la simetría $a_{i j}=a_{j i}$ podemos escribir

$$
\ddot{q}^{i}+\frac{1}{2} a^{i l}\left[\partial_{j} a_{k l}+\partial_{k} a_{l j}-\partial_{l} a_{j k}\right] \dot{q}^{j} \dot{q}^{k}=-a^{i l} \partial_{l} V,
$$

que es la expresión para las ecuaciones de Euler-Lagrange (2.3) expresada en términos de la matriz de masa $a_{i j}$. 
Observación:

Si la matriz de masa $a_{i j}=\delta_{i j}$ (matriz identidad) obtenemos las ecuaciones de Newton

$$
\ddot{q}^{i}=-a^{i l} \partial_{l} V
$$

\subsection{Formulación hamiltoniana}

Podemos considerar otro enfoque algo más sofisticado conceptualmente.

En principio vamos a considerar una variedad $(2 n+1)$-dimensional donde el tiempo será considerado además de un parámetro una coordenada, así las coordenadas de esta variedad serán $\left(t, x^{j}\right)$.

Sea la función lagrangiana

$$
L=L(\mathbf{q}(t), \dot{\mathbf{q}}(t), t), \quad \text { con } \dot{\mathbf{q}}(t)=\frac{d \mathbf{q}}{d t},
$$

sigamos el siguiente proceso:

1.- Identificamos

$$
p_{k} \longleftrightarrow a_{k l} \frac{d q^{l}}{d t}=\frac{\partial L(t, \mathbf{q}, \dot{\mathbf{q}})}{\partial \dot{q}^{k}}
$$

siendo una biyección bien definida al ser la matriz de masa $a_{i j}$ definida positiva.

2.- Definimos la función de Hamilton como sigue (via transformada de Legendre):

$$
H\left(t, q^{h}, p_{h}\right)=-L\left[t, q^{h}, \phi^{h}\left(t, q^{l}, p_{l}\right)\right]+p_{j} \phi^{j}\left(t, q^{h}, p_{h}\right)
$$

con $\dot{q}^{j}=\phi^{j}\left(t, q^{h}, p_{h}\right)=a^{j l}(\mathbf{q}) p_{l}$.

Tenemos la siguiente identificación

(Tensor/vector covariante) $p_{j} \longleftrightarrow \dot{q}^{j}$ (Tensor/vector contravariante)

Espacio de fases $\left(q^{j}, p_{j}\right) \longleftrightarrow\left(q^{j}, \dot{q}^{j}\right)$ Espacio de configuración

Por tanto trabajaremos en el fibrado cotangente $T^{*} Q=\bigcup_{p \in Q}\{p\} \times T_{p}^{*} Q$. 
Si tenemos una función lagrangiana de la forma (2.5), entonces el correspondiente hamiltoniano será

$$
H=H(\mathbf{q}, \mathbf{p})=\frac{1}{2} m a^{i j}(\mathbf{q}) p_{i} p_{j}+V(\mathbf{q}) .
$$

Pueden comprobarse fácilmente las siguientes identidades

$$
\begin{gathered}
\frac{d q_{k}}{d t}=\frac{\partial H}{\partial p_{k}}, \\
\frac{\partial H}{\partial q^{j}}=-\frac{\partial L}{\partial q^{j}} .
\end{gathered}
$$

Reescribiendo las ecuaciones de Euler-Lagrange (2.3) tenemos

$$
E_{l}(L)=\frac{d}{d t}\left(\frac{\partial L}{\partial \dot{q}^{l}}\right)-\frac{\partial L}{\partial q^{l}}=\frac{d p_{l}}{d t}+\frac{\partial H}{\partial q^{l}}=0
$$

Por tanto, las trayectorias (su evolución temporal) en el espacio de fases $(\mathbf{q}(t), \mathbf{p}(t))$ serán las soluciones del siguiente sistema de ecuaciones diferenciales ordinarias de primer orden (ecuaciones de Hamilton)

$$
\begin{aligned}
\frac{d p_{i}}{d t} \equiv \dot{p}_{i} & =-\frac{\partial H}{\partial q^{i}} \quad(i=1, \ldots, n), \\
\frac{q^{i}}{d t} \equiv \dot{q}^{i} & =\frac{\partial H}{\partial p_{i}}
\end{aligned}
$$

con $q^{i}=$ coordenadas generalizadas y $p_{i}=$ momentos conjugados.

Los pares de variables $\left(q^{i}, p_{i}\right)$ se llaman pares canónicamente conjugados y las ecuaciones 2.19) ecuaciones canónicas.

Puede que la función de Hamilton dependa del tiempo, es decir, que tenga la forma

$$
H=H(q, p, t) \text {. }
$$

En este caso podemos expandir el espacio de fases introduciendo dos nuevas coordenadas canónicas

$$
\begin{array}{rlr}
q^{0} & = & t \\
p_{0} & = & -H
\end{array}
$$

con el nuevo hamiltoniano

$$
H^{\prime}=H\left(q, p, q^{0}\right)+p_{0}
$$


Las ecuaciones de movimiento en el espacio ampliado serán entonces

$$
\begin{aligned}
\frac{d p_{i}}{d t} & =-\frac{\partial H^{\prime}}{\partial q^{i}} \\
\frac{d q^{i}}{d t} & =\frac{\partial H^{\prime}}{\partial p_{i}} \quad(i=0, \ldots, n)
\end{aligned}
$$

tendríamos que

$$
\begin{aligned}
& \dot{p}_{0}=-\frac{\partial H^{\prime}\left(q, p, q^{0}\right)}{\partial q^{0}}=\frac{\partial H(q, p, t)}{\partial t} \\
& \dot{q}^{0}=\frac{\partial H^{\prime}\left(q, p, q^{0}\right)}{\partial p_{0}}=1
\end{aligned}
$$

La primera ecuación implica que si el hamiltoniano $H$ no depende del tiempo, entonces $H=$ cte.. La segunda ecuación refleja que el tiempo es una coordenada y que su evolución es lineal. Por tanto en función de cómo hemos definido las nuevas coordenadas se cumple que $p_{0}=-H=$ cte. y no contiene información por lo que podemos describir las propiedades del sistema dinámico expresándolo únicamente en las coordenadas canónicas

$$
\left(q^{0}=t, q^{1}, \ldots, q^{n}, p_{1}, \ldots, p_{n}\right)
$$

De alguna forma podemos considerar el sistema como un Hamiltoniano $H(p, q, t)$ con $n+\frac{1}{2}$ grados de libertad.

Podríamos definir un elemento diferencial de volumen como

$$
d V=d \mathbf{p} d \mathbf{q}=d p_{1} \ldots d p_{n} d q^{1} \ldots d q^{n}
$$

así el volumen para el hipervolumen $S$ sería

$$
V=\int_{\partial S} d V
$$

Un conocido resultado debido a Liouville afirma que el volumen definido en el espacio de fases es conservado por la dinámica del sistema para dos momentos arbitrarios de tiempo. El flujo cuyas líneas de corriente fueran sus trayectorias es incompresible. Esta conservación de volumen excluye la posibilidad de puntos asintóticamente estables, esto es, no puede haber atractores en los sistemas Hamiltonianos.

\subsection{Formulación simpléctica}

Definición Una aplicación bilineal $\omega \in L^{2}(V, \mathbb{R})(\omega: V \times V \longrightarrow \mathbb{R})$ es no degenerada si $\omega(\mathbf{v}, \mathbf{w})=0$ para todo $\mathbf{w} \in V$ entonces $\mathbf{v}=0$. 
Definición Si $V$ es espacio vectorial real de dimensión finita y $\omega$ una aplicación bilineal antisimétrica en $V$, entonces $\omega$ es no degenerada si y sólo si $V$ tiene dimensión par y $\omega^{n}=\omega \wedge \cdots \wedge \omega$ es un volumen en $V$.

Por tanto si nuestro espacio vectorial $V$ tiene dimensión $2 n$ puedo elegir $\mathrm{n}$ elementos de la base de $V$, digamos $B=\left\{\mathbf{e}_{\mathbf{1}}, \ldots, \mathbf{e}_{\mathbf{n}}\right\}$ y otros n elementos su base dual canónica, digamos $B^{*}=\left\{\mathbf{e}^{\mathbf{n}+\mathbf{1}}, \ldots, \mathbf{e}^{\mathbf{2 n}}\right\}$ tales que

$$
\omega=\mathbf{e}^{1} \wedge \mathbf{e}^{\mathrm{n}+1}+\cdots+\mathrm{e}^{\mathrm{n}} \wedge \mathrm{e}^{2 \mathrm{n}},
$$

de tal manera que $\omega$ tendrá por matriz

$$
\left(\begin{array}{cc}
0 & I_{n} \\
-I_{n} & 0
\end{array}\right)
$$

Proposición Sea $(M, \omega)$ una variedad simpléctica. Entonces se cumple:

(i) $\operatorname{dim} M=2 n$

(ii) es orientable y podemos definir el volumen

$$
V=\frac{(-1)^{\frac{n(n+1)}{2}}}{n !} \omega^{n}
$$

(iii) Si $f: M \rightarrow M$ es una aplicación simpléctica entonces $f$ conserva el volumen.

En muchos problemas de mecánica el espacio de fases es el fibrado cotangente $T^{*} Q=\bigcup_{p \in Q}\{p\} \times T_{p}^{*} Q$ de un cierto espacio de configuración $Q$.

Si el espacio de configuración $Q$ tiene estructura de variedad diferenciable ndimensional podemos determinar un sistema de coordenadas locales, digamos $\left\{q^{1}, \ldots, q^{n}\right\}$.

A continuación, de manera natural, podemos determinar en un punto $q \in Q$ una base del espacio cotangente (dual) $T_{q}^{*} Q$ como $d q^{1}, \ldots, d q^{n}$. De esta manera cualquier 1-forma $\alpha$ definida en este espacio cotangente en $\mathbf{q}$, esto es, en $T_{q}^{*} Q$ se puede escribir como la combinación lineal $\alpha=p_{i} d q^{i}$ por lo que podemos poner coordenadas locales al fibrado cotangente $T^{*} Q$ de la forma $\left\{q^{1}, \ldots, q^{n}, p_{1}, \ldots, p_{n}\right\}$. Sobre esta variedad 2 n-dimensional sobre la que definiremos una 2-forma antisimétrica no degenerada, es decir, la estructura simpléctica

$$
\omega=d p_{i} \wedge d q^{i}
$$

con

$$
\omega(\mathbf{q}, \mathbf{p})\left(T_{(\mathbf{q}, \mathbf{p})}\left(T^{*} Q\right), T_{(\mathbf{q}, \mathbf{p})}\left(T^{*} Q\right)\right) .
$$


Una vez fijada la estructura, debemos establecer las transformaciones que preservan esta estructura, por así decirlo las transformaciones permitidas en mi problema mecánico, que, evidentemente, serán aquellas que preserven la estructura simpléctica llamadas simplectomorfismos, esto es transformaciones canónicas sobre $T^{*} Q$.

Hay una manera de crear transformaciones canónicas en $T^{*} Q$ a partir de difeomorfismos del espacion de configuración $f: Q \rightarrow Q$. Este proceso se denomina elevación de modo, que la definimos de la siguiente manera

$$
T^{*} f: T^{*} Q \rightarrow T^{*} Q
$$

definida como

$$
\left[\left(T^{*} f\right)\left(\alpha_{q}\right)\right](\mathbf{v})=\alpha_{q}(T f(v))
$$

donde $\alpha_{q}=T_{q}^{*} Q$ y $v \in T_{f^{-1}(q)} Q$ asegurándonos que $T^{*} f$ es simpléctica,

A partir de la identificación anterior el momento conjugado queda definido el espacio de fases de un sistema mecánico con coordenadas locales $\left(q^{i}, p_{i}\right)$, siendo este una variedad simpléctica donde las trayectorias o flujos de la evolución temporal de un sistema dinámico conservativo representado por las ecuaciones de Hamilton es una familia uniparamétrica de difeomorfismos simplécticos.

El espacio de fases es el fibrado cotangente $T^{*} Q$ del espacio de configuración $Q$.

Si asumimos que $Q$ es una variedad n-dimensional con coordenadas locales $\left\{q^{1}, \ldots, q^{n}\right\}$ entonces de manera natural una base del dual a su espacio tangente en $q$ denotado por $T_{q}^{*} Q$ será $\left\{d q^{1}, \ldots, d q^{n}\right\}$ por lo que cualquier 1-forma $\alpha \in T_{q}^{*} Q$ puede expresarse como $\alpha=p_{i} d q^{i}$, por tanto podemos definir las coordenadas locales s $T^{*} Q$ como $\left\{q^{1}, \ldots, q^{n}, p_{1}, \ldots, p_{n}\right\}$.

Así podemos determinar localmente coordenadas llamadas canónicas o simplécticas en el que la forma simpléctica toma la forma $\omega=d p_{i} \wedge d q^{i}$ donde $\omega$ es localmente constante, por tanto $d \omega=0$.

Definición Llamamos Sistema mecánico Hamiltoniano a la terna $(M, \omega, H)$ donde $(M, \omega)$ es una variedad simpléctica y $H \in C^{\infty}(M, \mathbb{R})$ una función real definida en $M$ llamada función energía.

Llamaremos campo vectorial Hamiltoniano $X_{H}$ a aquel que cumple

$$
i_{X_{H}} \omega+d H=0 .
$$


Proposición Sean $\left\{q^{1}, \ldots, q^{n}, p_{1}, \ldots, p_{n}\right\}$ coordenadas canónicas de $(M, \omega)$ y por tanto, $\omega=d p_{i} \wedge d q_{i}$. Entonces en estas coordenadas el campo vectorial $X_{H}$ que cumple la condición 2.35 es

$$
X_{H}=\sum_{i=1}^{n} \frac{\partial H}{\partial p_{i}} \frac{\partial}{\partial q^{i}}-\frac{\partial H}{\partial q^{i}} \frac{\partial}{\partial p_{i}}
$$

Proposición La curva integral $\left(q^{i}(t), p_{i}(t)\right)$ del campo vectorial $X_{H}$ debe cumplir

$$
\begin{aligned}
\dot{q}^{i} & =\frac{\partial H}{\partial p_{i}} \\
\dot{p}_{i} & =-\frac{\partial H}{\partial q^{i}} \quad i=1,2, \ldots, n
\end{aligned}
$$

denominadas ecuaciones de Hamilton.

Proposición Sea $(M, \omega, H)$ un sistema mecánico Hamiltoniano y $c(t)$ la curva integral de $X_{H}$ entonces $H(c(t))=$ constante a lo largo de la curva, esto es, si $\phi_{t}$ es el flujo de $X_{H}$, entonces $H \circ \phi_{t}=H$.

Proposición Sea $(M, \omega, H)$ un sistema mecánico hamiltoniano y $\phi_{t}$ el flujo de $X_{H}$. Entonces para cada $t, \phi_{t}^{*} \omega=\omega$, por tanto, $\phi_{t}$ es simpléctica y por tanto conserva el volumen $V_{\omega}$.

A la hora de generalizar el concepto, supongamos tenemos coordenadas locales $\left\{z^{1}, \ldots, z^{2 n}\right\}$ en un espacio de fases $2 n$-dimensional.

Sean $f, g \in C^{\infty}(M, \mathbb{R})$. Definimos

$$
\{f, g\}=g_{i k} \frac{\partial f}{\partial z^{i}} \frac{\partial g}{\partial z^{k}}
$$

donde $g_{i j\left(z^{i}\right)}$ tiene naturaleza tensorial.

\section{Propiedades}

a) Bilinealidad

$\{a f(z)+b g(z), h(z)\}=a\{f(z), h(z)\}+b\{g(z), h(z)\}$ con $a, b=$ constantes.

b) Antisimetría

$\{f(z), h(z)\}=-\{h(z), f(z)\}$. 
c) Igualdad de Leibniz

$\{f(z) g(z), h(z)\}=g(z)\{f(z), h(z)\}+f(z)\{g(z), h(z)\}$.

d) Igualdad de jacobi

$$
\{f(z),\{g(z), h(z)\}\}+\{h(z),\{f(z), g(z)\}\}+\{g(z),\{h(z), f(z)\}\}=0 .
$$

Si el sistema se describe a partir de la función $H(z)$, se denomina sistema Hamiltoniano generalizado y sus ecuaciones de movimiento se escriben como

$$
\dot{z}^{i}=\left\{z^{i}, H\right\}(i=1, \ldots, 2 n)
$$

Podemos escribir la variación de una propiedad $A(z)$ con el tiempo como

$$
\frac{d A}{d t}=\frac{\partial A}{\partial z^{i}} \dot{z}^{i}=\frac{\partial A}{\partial z^{i}}\left\{z^{i}, H\right\}=\{A, H\}
$$

En particular si

$$
g_{i j}=\left(\begin{array}{cc}
0 & I_{n} \\
-I_{n} & 0
\end{array}\right)
$$

con

$\left\{z^{1}, \ldots, z^{n}\right\}=\left\{q^{1}, \ldots, q^{n}\right\}$

$\left\{z^{n+1}, \ldots, z^{2 n}\right\}=\left\{p_{1}, \ldots, p_{n}\right\}$

obteniendo las mismas ecuaciones que (2.37).

Definición Sea $(M, \omega)$ una variedad simpléctica y $f, g \in C^{\infty}(M, \mathbb{R})$. El corchete de Poisson de $f$ y $g$ se define como

$$
\{f, g\}_{\omega}=-\omega\left(X_{f}, X_{g}\right)
$$

Proposición Sea $(M, \omega)$ una variedad simpléctica. En coordenadas canónicas $q^{1}, \ldots, q^{n}, p_{1}, \ldots, p_{n}$ se cumple

$$
\{f, g\}_{\omega}=\left(\frac{\partial f}{\partial q^{i}} \frac{\partial g}{\partial p_{i}}-\frac{\partial f}{\partial p_{i}} \frac{\partial g}{\partial q^{i}}\right) .
$$

Definición Sea $(M, \omega, H)$ un sistema mecánico hamiltoniano. Una función $f \in C^{\infty}(M, \mathbb{R})$ se dice constante de movimiento o integral si cumple

$$
\{f, H\}_{\omega}=0 .
$$

Sea $(M, \omega, H)$ un sistema mecánico Hamiltoniano. Sea $e \in \mathbb{R}$ un valor regular de $H$, esto es $d H(z) \neq 0$ si $x \in H^{-1}(e)$, por tanto $H^{-1}(e)$ es una subvaiedad 
de $M$ de codimensión 1.

Si escribimos $\Sigma_{e}$ la componente conexa de $H^{-1}(e)$, sabemos que $\Sigma_{e}$ es una superficie de energía regular.

Las curvas integrales de $X_{H}$ con condiciones iniciales en $\Sigma_{e}$ permanece en $\Sigma_{e}$, esto es, $X_{H}$ es tangente a $\Sigma_{e}$.

\subsection{Transformaciones canónicas. Función generatriz}

Supongamos que tenemos las variables canónicas $\left(q^{i}, p_{i}\right)$ con las ecuaciones de movimiento de Hamilton

$$
\begin{aligned}
& \dot{q}^{i}=\frac{\partial H}{\partial p_{i}} \\
& \dot{p}_{i}=-\frac{\partial H}{\partial q^{i}},
\end{aligned}
$$

y queremos hacer la transformación canónica

$$
\begin{aligned}
Q^{i} & =Q^{i}(q, p, t) \\
P_{i} & =P_{i}(q, p, t),
\end{aligned}
$$

de tal manera que exista una función de Hamilton $K\left(Q_{i}, P_{i}, t\right)$ tal que las ecuaciones de movimiento de Hamilton en estas nuevas variables sean

$$
\begin{array}{llr}
\dot{Q}^{i} & =\frac{\partial K}{\partial P_{i}} \\
\dot{P}_{i} & = & -\frac{\partial K}{\partial Q^{i}}
\end{array}
$$

Queremos que se cumpla el nuevo problema variacional

$$
\delta \int_{t_{1}}^{t_{2}} L d t=0 \Leftrightarrow \delta \int_{t_{1}}^{t_{2}}\left(\dot{Q}_{i} \cdot P_{i}-K\left(Q^{i}, P_{i}, t\right)\right) d t=0
$$

El anterior problema variacional en las coordenadas originales era

$$
\delta \int_{t_{1}}^{t_{2}} L d t=0 \Leftrightarrow \delta \int_{t_{1}}^{t_{2}}\left(\dot{q}_{i} \cdot p_{i}-H\left(q^{i}, p_{i}, t\right)\right) d t=0
$$

Como en los extremos la variación debe de ser nula, los integrandos deben estar relacionados como 


$$
\lambda\left(\dot{q}^{i} p_{i}-H\right)=\dot{Q}^{i} P_{i}-K+\frac{d F}{d t}
$$

La función $F$ de denomina función generatriz de la transformación canónica.

Con $\lambda=$ cte., en el caso $\lambda=1$ se dice transformación canónica, es importante $F$ para obtener la expresión de la transformación canónica tan sólo cuando expreso $F$ en términos de la mitad de las coordenadas nuevas y la mitad de las antiguas.

Supongamos $F=F_{1}(q, Q, t)$. Entonces la relación (2.45) se expresa como

$\dot{q}^{i} p_{i}-H=\dot{Q}^{i} P_{i}-K+\frac{\partial F_{1}}{\partial q^{i}} \dot{q}^{i}+\frac{\partial F_{1}}{\partial Q^{i}} \dot{Q}^{i}+\frac{\partial F_{1}}{\partial t}$.

Como coordenadas antiguas y nuevas son linealmente independientes, se cumple

$$
\left(p_{i}-\frac{\partial F}{\partial q^{i}}\right) \dot{q}^{i}-H+K+\frac{\partial F_{1}}{\partial t}-\left(P_{i}+\frac{\partial F_{1}}{\partial Q^{i}}\right) \dot{Q}^{i}=0
$$

resultando

$$
\begin{aligned}
p_{i} & =\frac{\partial F_{1}}{\partial q^{i}} \\
P_{i} & =-\frac{\partial F_{1}}{\partial Q^{i}} \\
K & =H+\frac{\partial F_{1}}{\partial t}
\end{aligned}
$$

relaciones que definirán $p_{i}=p_{i}\left(q^{i}, Q^{i}, t\right)$.

También podríamos buscar una función generatriz de tipo

$$
F=F_{2}(q, Q, t)-Q^{i} P_{i}
$$

obteniendo las relaciones

$$
\begin{aligned}
p_{i} & =\frac{\partial F_{2}}{\partial q^{i}} \\
Q^{i} & =\frac{\partial F_{2}}{\partial P_{i}} \\
K & =H+\frac{\partial F_{2}}{\partial t} .
\end{aligned}
$$

Igualmente con

$$
\begin{gathered}
F=F_{3}(p, Q, t)+q^{i} p_{i} \\
F=F_{4}(p, P, t)-Q^{i} P_{i}+q^{i} p_{i}
\end{gathered}
$$




\subsection{Ecuación de Hamilton-Jacobi}

Cuando hacemos una transformación canónica $\left(q^{i}, p_{i}\right) \longrightarrow\left(\mathrm{Q}^{i}, P_{i}\right)$, podríamos asegurar que las nuevas coordenadas fueran constantes de movimientoas. Las ecuaciones de movimiento serán

$$
\begin{aligned}
& \frac{\partial K}{\partial P_{i}}=\dot{Q}^{i}=0 \\
& \frac{\partial K}{\partial Q^{i}}=-\dot{P}_{i}=0
\end{aligned}
$$

además la relación entre ambos hamiltonianos, antiguo $H$ y nuevo $K$ y la función generatriz, tendrá la forma

$$
K=H+\frac{\partial F}{\partial t}
$$

y, por tanto,

$$
H(q, p, t)+\frac{\partial F}{\partial t}=0 .
$$

Si consideramos la función generatriz $F_{2}=F_{2}(q, P, t)$ se cumpliría que $p_{i}=\frac{\partial F_{2}}{\partial q^{i}}$. Así obtenemos

$$
H\left(q^{1}, q^{2}, \ldots, q^{n} ; \frac{\partial F_{2}}{\partial q^{1}}, \ldots, \frac{\partial F_{2}}{\partial q^{n}} ; t\right)+\frac{\partial F_{2}}{\partial t} .
$$

Esta ecuación se llama ecuación de Hamilton-Jacobi, que es una ecuación en derivadas parciales para la función generatriz $F_{2}$. La solución de (2.55) recibe el nombre de función principal de Hamilton.

Supongamos que exista una solución de 2.55 de la forma

$$
F_{2} S=S\left(q^{1}, q^{2}, \ldots, q^{n} ; \alpha_{1}, \ldots, \alpha_{n} ; t\right) .
$$

Podríamos tomar para las $n$ constantes de integración las nuevas coordenadas de movimiento, es decir, $P_{i}=\alpha_{i}$.

Así, a partir de las propiedades de la función generatriz $F_{2}$, sabemos que

$$
\begin{array}{ccc}
p_{i} & =\frac{\partial S(q, \alpha, t)}{\partial q^{i}} \\
Q^{i}=\beta_{i} & =\frac{\partial S(q, \alpha, t)}{\partial \alpha_{i}} .
\end{array}
$$

Podemos invertir estas ecuaciones para obtener

$$
\begin{gathered}
q_{j}=q_{j}(\alpha, \beta, t) \\
p_{i}=p_{i}(\alpha, \beta, t),
\end{gathered}
$$


que serán las soluciones a las ecuaciones de Hamilton.

\subsection{Variables acción-ángulo}

Supongamos un sistema conservativo de un grado de libertad con hamiltoniano

$$
H(q, p)=E,
$$

de donde podríamos despejar el momento conjugado $p=p(q, E)$.

Podemos introducir una nueva variable de acción I como

$$
I=\oint p d q
$$

con la integración extendida a todo el periodo de libración o rotación. Se puede deducir de 2.60 que $I=I(E)$ y, por tanto,

$$
E \equiv H=H(I)
$$

Se puede demostrar que cualquier Hamiltoniano integrable es sólo función de la coordenada de acción siempre y cuando las variables $(q, p)$ sean canónicamente conjugadas. Definimos

$$
S(q, I)=\oint p(q, H(I)) d q
$$

$\mathrm{S}$ es una función generatriz para la transformación canónica de tipo $F_{2}$ cumpliendo

$$
\theta=\frac{\partial S(q, I)}{\partial I}
$$

Por tanto hemos conseguido el par de coordenadas canónicamente conjugado $(I, \theta)$ que cumplirán las ecuaciones de Hamilton

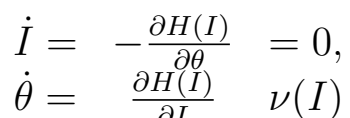

siendo $\nu(I)$ la frecuencia del movimiento periódico, por tanto

$$
\begin{gathered}
I=c t e \\
\theta=\nu(I) t+\theta_{0}
\end{gathered}
$$




\subsubsection{Movimiento multidimensional}

Consideremos un sistema integrable multidimensional dado por el hamiltoniano

$$
h=H(q, p)=H\left(q^{1}, \ldots, q^{n}, p_{1}, \ldots, p_{n}\right)
$$

Supongamos que este sistema hamiltoniano tienen integrales primeras $F_{1}=$ $H, F_{2}, \ldots, F_{n}$ tales que

$$
\begin{aligned}
& F_{i}=F_{i}(q, p), \quad i=1, \ldots, n, \\
& \left\{F_{i}, H\right\}_{\omega}=0, \quad i=1, \ldots, n,
\end{aligned}
$$

conmutan entre sí, (en involución), es decir,

$$
\left\{F_{i}, F_{i}\right\}_{\omega}=0, \quad i=1, \ldots, n
$$

y

$$
\left\{d F_{1}, \ldots, d F_{n}\right\}
$$

son linealmente independientes.

Entonces se cumple que:

1. Las trayectorias del sistema se encuentran sobre la superficie de un toro 2n-dimensional.

2. El movimiento es periódico y se caracteriza por las $n$ frecuencias

$$
\nu_{i}=\nu_{i}\left(F_{1}, \ldots, F_{n}\right) \quad i=1, \ldots, n
$$

3. La variables angulares $\theta_{i}=\nu_{i} t+\theta_{i 0}, \operatorname{con} i=1, \ldots, n$ y $\theta_{i 0}$ constante de integración.

Durante el movimiento, la trayectoria no abandona la superficie de toro.

Para $n=2$ los toros correspondientes a diferentes valores de las integrales $\left(F_{1}, F_{2}\right)$ están anidados, unos sobre otros, sin cortarse dividiendo el espacio. El movimiento en la superficie de los toros de dos dimensiones se caracteriza por dos frecuencias $\nu_{1}$ y $\nu_{2}$. En general las frecuencias son inconmensurables entre sí, es decir, $\frac{\nu_{1}}{\nu_{2}} \neq \frac{m_{1}}{m_{2}}$. Por tanto, la trayectoria no es periódica y no vuelve nunca al mismo punto del que partió pero cubre densamente el toro, por tanto, se acercará al punto inicial tanto como queramos asintóticamente. 
Pero si la relación de frecuencias es un número racional, es decir,

$$
\frac{\nu_{1}}{\nu_{2}}=\frac{m_{1}}{m_{2}}, \text { con } m_{1} \text { y } m_{2} \text { enteros }
$$

se dice que estamos en una resonancia.

La trayectoria vuelve al punto inicial tras un número finito de vueltas alrededor del toro.

La condición de resonancia viene dada por la relación

$$
\sum_{i=1}^{n} m_{i} \nu_{i}=0
$$

con $m_{i} \in \mathbb{Z}$ distintos de cero.

Nos gustaría definir $n$ variables de acción-ángulo, para definir las variables de acción dado que el movimiento se realiza sobre un toro n-dimensional $\mathbb{T}^{n}$, en el caso $n=2$ podemos elegir dos contornos $C_{j}$ de $\mathbb{T}^{2}$ que no puedan ser transformados entre sí ni reducidos a un punto, que podrán ser las circunferencias cuyo producto directo es el toro $\mathbb{T}^{2}$.

En el caso de dimensión $n$ podemos definir n variables de acción como

$$
I_{j}=C_{j} \sum_{i=1}^{n} p_{i} d q^{i}, i=1, \ldots, n,
$$

que son integrales de movimiento.

Podemos expresar las antiguas $F_{i}$ en término de las acciones $I_{j}$ como

$$
F_{i}=F_{i}\left(I_{1}, \ldots, I_{n}\right) i=1, \ldots, n
$$

Igualmente el hamiltoniano se puede expresar en términos únicamente de las variables de acción

$$
H=H\left(I_{1}, \ldots, I_{n}\right) \text {. }
$$

Las ecuaciones de Hamilton tendrán la forma

$$
\begin{aligned}
\dot{I}_{j} & =-\frac{\partial H}{\partial \theta_{i}} \\
\dot{\theta}_{j} & =\frac{\partial H}{\partial I_{i}}
\end{aligned}
$$


donde

$$
\begin{gathered}
I_{j}=c t e . \\
\theta_{j}=\nu_{j} t+c t e . \\
\nu_{i}=\nu_{i}\left(I_{1}, \ldots, I_{n}\right)=\frac{\partial H}{\partial I_{n}} i=1, \ldots, n
\end{gathered}
$$

Si el sistema es no degenerado, es decir, si cumple

$$
\left\|\frac{\partial \nu_{i}(I)}{\partial I_{j}}\right\|=\left\|\frac{\partial^{2} H(I)}{\partial I_{j} I_{i}}\right\| \neq 0
$$

entonces los toros invariantes tienen la forma

$$
(I)=\left(I_{1}, \ldots, I_{n}\right)=\text { cte. }
$$

Podemos analizar las propiedades espectrales de las trayectorias del sistema. Para ello, necesitamos conocer

$$
\begin{gathered}
q=q(I, \theta) \\
p=p(I, \theta) .
\end{gathered}
$$

Podemos desarrollar en serie de Fourier cada variable

$$
\begin{gathered}
q=\sum_{m=-\infty}^{\infty} q_{m}(I) e^{i \boldsymbol{m} \boldsymbol{\theta}} \\
p=\sum_{m=-\infty}^{\infty} p_{m}(I) e^{i \boldsymbol{m} \boldsymbol{\theta},}
\end{gathered}
$$

siendo

$$
\begin{aligned}
\boldsymbol{m} & =\left(m_{1}, \ldots, m_{n}\right) \\
\boldsymbol{\theta} & =\left(\theta_{1}, \ldots, \theta_{n}\right),
\end{aligned}
$$

$\operatorname{con} m_{i} \in \mathbb{Z}$.

se puede escribir en términos de $(I, \nu)$

$$
\begin{gathered}
q=\sum_{m=-\infty}^{\infty} \widetilde{q}_{m}(I) e^{i m \nu t} \\
p=\sum_{m=-\infty}^{\infty} \widetilde{p}_{m}(I) e^{i m \nu t}
\end{gathered}
$$

$\operatorname{con} \boldsymbol{\nu}=\left(\nu_{1}, \ldots, \nu_{n}\right)$.

Si se satisface la condición (2.80), la trayectoria (2.85) describe un movimiento $n$-frecuencial, si cumplen $\sum_{i=1}^{n} m_{i} \nu_{i}=0$ el movimiento será periódico. 


\section{Capítulo 3}

\section{Formalismo geometrodinámico}

\subsection{Proceso de geometrización y Métrica de Jacobi}

En los desarrollos anteriores no hubo ninguna referencia a conceptos métricos, esto es, las soluciones de las ecuaciones de Euler-Lagrange son meras trayectorias del sistema dinámico definido en el espacio de configuración o fibrado tangente obtenidas a partir del Principio de acción estacionaria definido para una determinada función Lagrangiana.

Por otro lado, las trayectorias solución de las ecuaciones de Hamilton son igualmente trayectorias del sistema definidas en el espacio de fases o fibrado cotangente. En este espacio hay definida de manera natural una estructura simpléctica $\omega=d p_{i} \wedge d q^{i}$ que es preservada por el flujo obtenido por estas trayectorias preservando así el volumen en el espacio de fases.

En adelante, asignaremos a la matriz de masa significado geométrico, entendiendo que ésta representa las componentes de cierto tensor métrico $a_{i j}(\mathbf{q})$. Este tensor, definido en el espacio de configuración, que representa la manera de medir la longitud del vector velocidad independientemente del potencial $V$. De esta forma, describe la cinemática del problema dinámico, por lo que tiene carácter meramente descriptivo. Al depender esta matriz de masa del punto $q^{i}$, puede originar que esté representando una métrica no euclídea, en particular, elíptica.

El planteamiento geométrico anterior permite aplicar el Principio de acción 
estacionaria sobre la siguiente integral de acción

$$
S[\gamma]=\int_{t_{1}}^{t_{2}}\left(\frac{1}{2}\|\dot{\gamma}\|^{2}-V(\gamma(t))\right) d t,
$$

por lo que

$$
\delta S=0 \Rightarrow \frac{D \dot{\gamma}}{d t}=-\operatorname{grad} V
$$

con $\frac{D}{d t} \equiv$ derivada covariante, que es la proyección sobre el espacio tangente de la segunda derivada del vector velocidad (aceleración).

Entendiendo el problema dinámico de esta forma, podemos calcular los Símbolos de Christoffel para esta métrica $a_{i j}(\mathbf{q})$, cuyo resultado coincide con el segundo término del primer miembro de (2.11)

$$
\Gamma_{j k}^{i}=\frac{1}{2} a^{i l}\left[\partial_{j} a_{k l}+\partial_{k} a_{l j}-\partial_{l} a_{j k}\right] .
$$

De esta forma, las ecuaciones de Euler-Lagrange pueden escribirse como

$$
\ddot{q}^{i}+\Gamma_{j k}^{i} \dot{q}^{j} \dot{q}^{k}=-a^{i l} \partial_{l} V
$$

Si al espacio métrico anterior, le aplicamos una transformación conforme (preserva ángulos, no distancias) especial, podemos obtener otra variedad riemanniana con un tensor métrico (métrica de Jacobi) que en coordenadas se define como

$$
\mathbf{g}=2(E-V(\mathbf{q})) a_{i j} d q^{i} \otimes d q^{i}, \text { de componentes } g_{i j}=2(E-V(\mathbf{q})) a_{i j}(\mathbf{q})
$$

con $E$ 三energía total (constante) y, $V(\mathbf{q}) \equiv$ energía potencial.

Calculamos las geodésicas (generalización de las líneas rectas en el espacio euclídeo) en este espacio, que deben cumplir

$$
\nabla_{\dot{\gamma}} \dot{\gamma}=0, \text { con } \nabla \equiv \text { conexión, }
$$

que en coordenadas adopta la forma

$$
\nabla_{\dot{\gamma}} \dot{\gamma}=\frac{d^{2} q^{i}}{d s^{2}}+\tilde{\Gamma}_{j k}^{i} \frac{q^{j}}{d s} \frac{q^{k}}{d s}=0
$$

donde $\tilde{\Gamma}_{j k}^{i}$ son los símbolos de Christoffel dados por 3.3 de la métrica $g_{i j}$ y $s \equiv$ parámetro longitud de arco. 


\subsubsection{Ecuaciones geodésicas con parámetro físico $(\mathrm{t})$}

La métrica Jacobi tiene la forma

$$
g_{i j}=2(E-V(q)) a_{i j}(q)
$$

Escribamos la diferencial de longitud en términos de diferenciales de tiempo físico

$$
d s=2(E-V(q)) d t \Rightarrow \frac{d t}{d s}=\frac{1}{2(E-V(q))}=\frac{1}{2 W}, \quad \text { con } W \equiv E-V(q) .
$$

Se tiene entonces

$$
\begin{gathered}
\frac{d q^{i}}{d s}=\frac{d q^{i}}{d t} \frac{d t}{d s}=\frac{d q^{i}}{d t} \frac{1}{2(E-V(q))} \\
\frac{d^{2} q^{i}}{d s^{2}}=\frac{d^{2} q^{i}}{d t^{2}} \frac{1}{(2 W)^{2}}+\frac{d q^{i}}{d t} \frac{1}{(2 W)^{2}}\left(\frac{\partial V}{\partial q^{l}} \frac{d q^{l}}{d t}\right) \frac{1}{W} .
\end{gathered}
$$

Sustituyendo en (3.7) obtenemos

$$
\frac{d^{2} q^{i}}{d t^{2}}+\frac{1}{W}\left(\frac{\partial V}{\partial q^{l}} \frac{d q^{l}}{d t}\right) \frac{d q^{i}}{d t}+\tilde{\Gamma}_{j k}^{i} \frac{d q^{j}}{d t} \frac{d q^{k}}{d t}=0
$$

Para poder comparar con las expresiones anteriores necesitamos expresar $\tilde{\Gamma}_{j k}^{i}$ (Símbolos de Christoffel de la métrica Jacobi $g_{i j}$ ) con $\Gamma_{j k}^{i}$ (Símbolos de Christoffel de la métrica $a_{i j}$ ). Para ello, apliquemos la expresión

$$
\begin{gathered}
\tilde{\Gamma}_{j k}^{i}=\frac{1}{2} g^{i l}\left[\partial_{j} g_{k l}+\partial_{k} g_{l j}-\partial_{l} g_{j k}\right] \\
=-\frac{1}{2 W}\left[\delta_{k}^{i} \partial_{j} V+\delta_{j}^{i} \partial_{k} V-\partial_{l} V a^{l i} a_{j k}\right]+\underbrace{\frac{1}{2} a^{i l}\left[\partial_{j} a_{k l}+\partial_{k} a_{l j}-\partial_{l} a_{j k}\right]}_{\Gamma_{j k}^{i}}
\end{gathered}
$$

de lo que se deduce que

$$
\begin{gathered}
\ddot{q}^{i}+\Gamma_{j k}^{i} \dot{q}^{j} \dot{q}^{k}=\frac{1}{2 W}\left[2 \partial_{j} V \dot{q}^{j} \dot{q}^{i}-\partial_{l} V a^{l i} 2 W\right]-\frac{1}{W} \partial_{l} V \dot{q}^{l} \dot{q}^{i} \Rightarrow \\
\ddot{q}^{i}+\Gamma_{j k}^{i} \dot{q}^{j} \dot{q}^{k}=-\partial_{l} V a^{i l},
\end{gathered}
$$

Que coincide con las ecuaciones de Euler-Lagrange (2.11), lo que indica es que estas soluciones no son meras trayectorias, sino que son las líneas rectas de la variedad riemanniana con métrica de Jacobi. 
Tabla 3.1: Equivalencia entre algunas magnitudes relevantes de la Dinámica y geometrodinámica de la Mecánica Clásica.

\begin{tabular}{ll}
\hline \hline Dinámica & Geometría \\
\hline Tiempo, $t$ & Longitud de arco, $s$ \\
Potencial, $V$ & Métrica, $g_{i j}$ \\
Fuerza, $-\partial V$ & Símbolos de Christoffel, $-\Gamma_{j k}^{i}$ \\
Trayectorias & Geodésicas \\
Cantidades conservadas & Simetrías \\
Estabilidad & Curvatura \\
\hline
\end{tabular}

Por tanto, dado que las trayectorias aparecen como las líneas más sencillas en esta variedad riemanniana, debemos entender que ésta, dotada con la métrica de Jacobi, es el dominio adecuado para estudiar el problema dinámico y la geometría diferencial intrínseca parece ser el lenguaje adecuado para dicho estudio.

El proceso de geometrización llevado a cabo se puede resumir como que la información acerca de la fuerza actuando sobre la partícula (descrita por $V(\mathbf{q})$ ) ha sido codificada plenamente en la definición de la métrica Jacobi (3,8) de una variedad riemanniana abstracta denominada variedad mecánica.

Básicamente, la energía se ha invertido en curvar el espacio euclídeo anterior de forma conforme para así generar una variedad riemanniana adecuada para que las geodésicas (líneas rectas) de este último espacio sean justamente las trayectorias evolución del sistema en el espacio de configuración.

Describiendo así no tanto la evolución temporal del sistema sino los puntos del espacio de configuración que constituyen dichas trayectorias.

Por tanto la trayectoria seguida por una partícula sometida a un potencial $V(\mathbf{q})$ en el espacio euclídeo equivale al movimiento de una partícula libre en la variedad mecánica.

Así, las trayectorias solución de las ecuaciones de Euler-Lagrange 2.3 son las geodésicas de la variedad riemanniana con la métrica de Jacobi (3.8). 


\subsection{Métrica Jacobi y óptica geométrica}

En este apartado mostramos la conexión entre un Principio óptico (Principio de Fermat) y otro mecánico (Principio de Maupertuis).

El Principio de Fermat afirma que la trayectoria de un rayo de luz será aquella que minimice el tiempo invertido en su recorrido.

Con el Principio de Hamilton nunca se podría obtener un resultado como el anterior ya que el tiempo permanece constante al variar las trayectorias. En cambio con el Principio de acción estacionaria hemos introducido a la vez la variación del tiempo.

Así el tiempo total de recorrido $t$ será

$$
\begin{gathered}
t=\int_{A}^{B} \frac{1}{v} d s=\underbrace{\int_{A, \gamma(t)}^{B} \frac{1}{v} \sqrt{\delta_{a b} \dot{q}^{a} \dot{q}^{b}} d t}_{\text {sobre trayectorias asíncronas }}= \\
=\int_{A, \gamma(t)}^{B} \sqrt{n^{2} \delta_{a b} \dot{q}^{a} \dot{q}^{b}} d t=\int_{A, \gamma(t)}^{B} \sqrt{g_{a b} \dot{q}^{a} \dot{q}^{b}} d t .
\end{gathered}
$$

Por tanto, encontrar la curva de tiempo mínimo entre los puntos $A$ y $B$ equivale a encontrar la curva que minimiza la distancia entre estos puntos en un espacio dotado de la métrica $g_{a b}=n^{2} \delta_{a b}$.

En el caso de un rayo de luz el índice de refracción será $n=\frac{1}{v}$. Sabemos que una partícula en un campo conservativo con energía $E$ y potencial $V(q)$ se mueve siguiendo geodésicas del espacio con métrica $g_{a b}=\frac{2}{m c^{2}}(E-V(q)) \delta_{a b}$, con $c=$ constante arbitraria con unidades de velocidad.

El movimiento de una partícula en un potencial es equivalente a la propagación de rayos de luz en un medio con índice de refracción

$$
n=\sqrt{\frac{2(E-V(q))}{m c^{2}}} .
$$

Esta analogía permite utilizar reflexión, refracción, interferencia,etc.

Si $m=c=1$ obtenemos la métrica de Jacobi

$$
g_{a b}=2(E-V(q)) \delta_{a b}=v^{2} \delta_{a b}
$$


Tabla 3.2: Comparativa entre métrica de Jacobi para la mecánica y la óptica.

\begin{tabular}{|c|c|c|}
\hline Tipo & Métrica & Índice de refracción \\
\hline Partículas & $g_{a b}=n^{2} \delta_{a b}=v^{2} \delta_{a b}$ & $n=v$ \\
\hline Luz & $g_{a b}=n^{2} \delta_{a b}=\frac{\delta_{a b}}{v^{2}}$ & $n=\frac{1}{v}$ \\
\hline
\end{tabular}

Trayectorias de partículas/rayos de luz serán equivalentes si definimos el índice de refracción $n$ y minimizamos la integral

$$
\int_{A, \gamma(t)}^{B} n d s
$$

Para una partícula con energía $E$ en un campo conservativo, $v=\sqrt{2(E-V(q))}$ Para un rayo de luz $n=\frac{1}{v}=$ índice de refracción del medio.

\subsection{Métrica Jacobi y Principio de Maupertuis}

La justificación teórica de estos hechos tiene su fundamento en el Principio de Maupertuis de acción estacionaria que afirma que los movimientos naturales serán aquellas trayectorias que minimicen la integral de acción

$$
\mathcal{A}=\int_{\gamma(t)} p_{i} d q^{i}=\int_{\gamma(t)} \frac{\partial L}{\partial \dot{q}^{i}} \dot{q}^{i} d t
$$

Llamando $W=\frac{\partial L}{\partial \dot{q}^{i}} \dot{q}^{i}$ a la energía cinética, la variación de 3.22

$$
\begin{aligned}
& \delta \mathcal{A}=\delta \int_{\gamma(t)} 2 W d t=\delta \int_{\gamma(t)} \sqrt{2 W} \sqrt{2 W} d t=\delta \int_{\gamma(t)} \sqrt{2(E-V(\mathbf{q}))} \sqrt{a_{i j} \dot{q}^{i} \dot{q}^{j}} d t= \\
& \delta \int_{\gamma(t)} \sqrt{2(E-V(\mathbf{q})) a_{i j} \dot{q}^{i} \dot{q}^{j}} d t=\int_{\gamma(s)} d s
\end{aligned}
$$

con $d s \equiv$ diferencial de longitud. Por tanto los movimientos naturales serán las geodésicas de la variedad riemanniana dotada con la métrica de Jacobi. 
Resumiendo la variedad mecánica consiste en una variedad riemanniana $\left(M, g_{J}\right)$ con $\mathbf{q}=\left(q^{1}, q^{2}, \ldots, q^{N}\right) \in M$ y $E=$ constante. Dotada con una métrica, tal que, en coordenados toma la forma

$$
g_{i j}=2(E-V(\mathbf{q})) a_{i j}(\mathbf{q})
$$

con $\left(a_{i j}\right)(\mathbf{q})$ la matriz de masa. Así la longitud de arco $s$ se expresa en términos del tiempo físico $(t)$ como

$$
s=\int 2(E-V(\mathbf{q})) d t \Rightarrow d s=2(E-V(\mathbf{q})) d t .
$$

Observación:

La métrica Jacobi es una transformación conforme de la métrica de espacio vacío $a_{i j}(q)$ de la forma

$$
g_{i j}=2(E-V(\mathbf{q})) a_{i j}(\mathbf{q})
$$

de tal manera que toda afirmación hecha en el espacio vacío en la que únicamente aparezca información angular, esto es, sin campo físico, será válida también añadiendo el campo físico $V(\mathbf{q})$.

\subsection{La métrica Eisenhart}

La variedad riemanniana $\left(M \times \mathbb{R}^{2}, g_{E}\right)$ con $\mathbf{q}=\left(q^{0}, q^{1}, q^{2}, \ldots, q^{N}, q^{N+1}\right) \in$ $M \times \mathbb{R}^{2}$ with $q^{0} \equiv t, q^{N+1} \in \mathbb{R}$

$$
\begin{gathered}
\left.d s^{2}=-2 V(\mathbf{q})\left(d q^{0}\right)^{2}+a_{i j}(\mathbf{q}) d q^{i} d q^{j}+2 d q^{0} d q^{N+1}, \quad \begin{array}{cccccc}
-2 V(\mathbf{q}) & 0 & 0 & \ldots & 0 & 2 \\
0 & a_{11} & a_{12} & \ldots & a_{1 N} & 0 \\
0 & a_{21} & a_{22} & \ldots & a_{2 N} & 0 \\
\vdots & \vdots & \vdots & & \vdots & 0 \\
0 & \left.a_{N 1}\right) & a_{N 2} & \ldots & a_{N N} & 0 \\
2 & 0 & 0 & \ldots & 0 & 0
\end{array}\right),(3,2 .,
\end{gathered}
$$


con $a_{i j}(\mathbf{q})$ la matriz de masa.

De entre todas las geodésicas generadas solamente consideramos aquellas para las que la longitud de arco es definida positiva, esto es,

$$
d s^{2}=2 C^{2} d t^{2}
$$

con $C=$ constante, podemos fijar el valor $C=\frac{1}{2}$.

Los símbolos de Christoffel para $N=2$ tienen la forma

$$
\begin{aligned}
& \Gamma_{00}^{1}=\frac{1}{a_{11}} \frac{\partial V}{\partial q^{1}}, \\
& \Gamma_{11}^{1}=\frac{1}{2 a_{11}} \frac{\partial a_{11}}{\partial q^{1}}, \\
& \Gamma_{12}^{1}=\frac{1}{2 a_{11}} \frac{\partial a_{11}}{\partial q^{2}}=\Gamma_{21}^{1}, \\
& \Gamma_{22}^{1}=-\frac{1}{2 a_{11}} \frac{\partial a_{22}}{\partial q^{1}}, \\
& \Gamma_{00}^{2}=\frac{1}{a_{22}} \frac{\partial V}{\partial q^{2}}, \\
& \Gamma_{11}^{2}=-\frac{1}{2 a_{22}} \frac{\partial a_{22}}{\partial q^{1}}, \\
& \Gamma_{12}^{2}=\frac{1}{2 a_{22}} \frac{\partial 22}{\partial q^{1}}=\Gamma_{21}^{2}, \\
& \Gamma_{22}^{2}=\frac{1}{2 a_{22}} \frac{\partial a_{22}}{\partial q^{2}} \\
& \Gamma_{01}^{3}=-\frac{\partial V}{\partial q^{1}}, \\
& \Gamma_{02}^{3}=-\frac{\partial V}{\partial q^{2}} .
\end{aligned}
$$

En particular si $a_{i j}=\delta_{i j}$ los símbolos de Christoffel no nulos tienen la forma

$$
\begin{aligned}
& \Gamma_{00}^{1}=\frac{\partial V}{\partial q^{1}}, \\
& \Gamma_{00}^{2}=\frac{\partial V}{\partial q^{2}}, \\
& \Gamma_{01}^{3}=-\frac{\partial V}{\partial q^{1}}, \\
& \Gamma_{02}^{3}=-\frac{\partial V}{\partial q^{2}} . \\
& \left(g^{i j}\right)=\left(\begin{array}{cccccc}
0 & 0 & 0 & \ldots & 0 & \frac{1}{2} \\
0 & 1 & 0 & \ldots & 0 & 0 \\
0 & 0 & 1 & \ldots & 0 & 0 \\
\vdots & \vdots & \vdots & & \vdots & 0 \\
0 & 0 & 0 & \ldots & 1 & 0 \\
\frac{1}{2} & 0 & 0 & \ldots & 0 & \frac{V(\mathbf{q})}{2}
\end{array}\right)
\end{aligned}
$$

Es sencillo comprobar que las ecuaciones de las geodésicas (3.7) con esta matriz de masa $\delta_{i j}$ dan como resultado la ecuaciones de Newton

$$
\frac{d^{2} q^{i}}{d t^{2}}=-\frac{\partial V}{\partial q^{i}}, \quad i=1,2, \ldots, N
$$


Calcularemos el tensor de curvatura y la curvatura escalar de esta métrica aplicando la ecuación (B.69) con los símbolos de Christoffel (3.30), que en coordenadas viene dado por

$$
\begin{aligned}
& R_{k l m}^{i}=\frac{\partial \Gamma_{k m}^{i}}{\partial x^{l}}-\frac{\partial \Gamma_{k l}^{i}}{\partial x^{m}}+\Gamma_{l s}^{i} \Gamma_{k m}^{s}-\Gamma_{m s}^{i} \Gamma_{k l}^{s}, \\
& R_{010}^{1}=\frac{1}{2 \cdot a_{11}^{2} \cdot a_{22}}\left[-a_{22} \frac{\partial a_{11}}{\partial q^{1}} \frac{\partial V}{\partial q^{1}}+a_{11}\left(\frac{\partial a_{11}}{\partial q^{2}} \frac{\partial V}{\partial q^{2}}+2 \cdot a_{22} \frac{\partial^{2} V}{\partial\left(q^{1}\right)^{2}}\right)\right], \\
& R_{020}^{1}=\frac{1}{2 \cdot a_{11}^{2}}\left[-\frac{\partial a_{11}}{\partial q^{2}} \frac{\partial V}{\partial q^{1}}+a_{11}\left(-\frac{1}{a_{22}} \frac{\partial V}{\partial q^{2}} \frac{\partial a_{22}}{\partial q^{1}}+2 \frac{\partial^{2} V}{\partial q^{1} \partial q^{2}}\right)\right], \\
& R_{221}^{1}=\frac{-1}{2 \cdot a_{11}^{2} \cdot a_{22}}\left[a _ { 1 1 } \left(\frac{\partial a_{11}}{\partial q^{2}} \frac{\partial a_{22}}{\partial q^{2}}+\right.\right. \\
& \left.\left.+\left(\frac{\partial a_{22}}{\partial q^{1}}\right)^{2}\right)+a_{22}\left(\left(\frac{\partial a_{11}}{\partial q^{2}}\right)^{2}+\frac{\partial a_{11}}{\partial q^{1}} \frac{\partial a_{22}}{\partial q^{1}}-2 a_{11}\left(\frac{\partial^{2} a_{11}}{\partial\left(q^{2}\right)^{2}}+\frac{\partial^{2} a_{22}}{\partial\left(q^{1}\right)^{2}}\right)\right)\right], \\
& R_{010}^{2}=\frac{1}{2 a_{22}^{2}}\left(-\frac{\partial V}{\partial q^{2}} \frac{\partial a_{22}}{\partial q^{1}}+a_{22}\left(\frac{-1}{a_{11}} \frac{\partial a_{11}}{\partial q^{2}} \frac{\partial V}{\partial q^{1}}+2 \frac{\partial^{2} V}{\partial q^{2} \partial q^{1}}\right)\right), \\
& R_{020}^{2}=\frac{1}{2 a_{22}^{2} a_{11}}\left[a_{11}\left(-\frac{\partial a_{22}}{\partial q^{2}} \frac{\partial V}{\partial q^{2}}+2 a_{22} \frac{\partial^{2} V}{\partial\left(q^{2}\right)^{2}}\right)+a_{22} \frac{\partial a_{22}}{\partial q^{1}} \frac{\partial V}{\partial q^{1}}\right], \\
& R_{110}^{3}=\frac{1}{2}\left[\frac{1}{a_{22}} \frac{-\partial a_{11}}{\partial q^{2}} \frac{\partial V}{\partial q^{2}}+\frac{1}{a_{11}} \frac{\partial a_{11}}{\partial q^{1}} \frac{\partial V}{\partial q^{1}}-2 \frac{\partial^{2} V}{\partial\left(q^{1}\right)^{2}}\right] \text {, } \\
& R_{120}^{3}=\frac{1}{2}\left[\frac{1}{a_{22}} \frac{\partial a_{22}}{\partial q^{1}} \frac{\partial V}{\partial q^{2}}+\frac{1}{a_{11}} \frac{\partial a_{11}}{\partial q^{2}} \frac{\partial V}{\partial q^{1}}-2 \frac{\partial^{2} V}{\partial q^{1} \partial q^{2}}\right], \\
& R_{220}^{3}=\frac{1}{2}\left[\frac{1}{a_{22}} \frac{\partial a_{22}}{\partial q^{2}} \frac{\partial V}{\partial q^{2}}-2 \frac{\partial^{2} V}{\partial\left(q^{2}\right)^{2}}-\frac{1}{a_{11}} \frac{\partial a_{22}}{\partial q^{1}} \frac{\partial V}{\partial q^{1}}\right] \text {. }
\end{aligned}
$$

Sabiendo que podemos subir o bajar índices de la siguiente forma $R_{j k l}^{i}=g^{i m} R_{m j k l}$ о $R_{m j k l}=g_{i m} R_{j k l}^{i}$.

Se define el tensor de Ricci $R_{l h}$ por contracción del tensor de curvatura $R_{l p h j}$ como

$$
R_{l h}=g^{p j} R_{l p h j}
$$

Hay un caso particular de espacios Riemannianos llamados espacio de Einstein que cumplen $R_{h l}=\lambda g_{h l}$ en particular $R_{h l}=\frac{R}{2} g_{h l}$ y de nuevo otra contracción define un invariante (excepto para $n=2$ ) llamado curvatura escalar

$$
R=R_{j}^{j}=g^{l j} R_{l j},
$$

donde se cumple que $\left.R\right|_{h}=0$, se puede demostrar que esta afirmación es cierta para todo espacio de Einstein de dimensión $n \neq 2$

$$
\begin{aligned}
& R_{11}=R_{22}=R_{33}=0, \\
& R=g^{l j} R_{l j}=R_{l}^{j}=g^{00} R_{00}=0 .
\end{aligned}
$$


Por tanto, cuando la matriz de masa $a_{i j}=\delta_{i j}$ el espacio es de curvatura escalar nula.

Calculamos la expresión de la curvatura escalar para esta métrica con matriz de masa $a_{i j}$ definida positiva, resultando

$$
\begin{gathered}
R=\frac{1}{2 \cdot a_{11}^{2} \cdot a_{22}^{2}}\left[a_{11}\left(\frac{\partial a_{11}}{\partial q^{2}} \frac{\partial a_{22}}{\partial q^{2}}+\left(\frac{\partial a_{22}}{\partial q^{1}}\right)^{2}\right)+\right. \\
a_{22}\left(\left(\frac{\partial a_{11}}{\partial q^{2}}\right)^{2}+\frac{\partial a_{11}}{\partial q^{1}} \frac{\partial a_{22}}{\partial q^{1}}-2 a_{11}\left\{\frac{\partial^{2} a_{11}}{\partial\left(q^{2}\right)^{2}}+\frac{\partial^{2} a_{22}}{\partial\left(q^{1}\right)^{2}}\right\}\right) .
\end{gathered}
$$

Es importante observar que la expresión para la curvatura escalar no depende del potencial, por tanto solamente refleja información del espacio con métrica identificada con la matriz de masa del problema dinámico.

Esta métrica es bastante utilizada en la bibliografía científica aunque es de poco interés matemático pues define una variedad riemanniana plana, esto es, de curvatura $R=0$.

\subsection{Ejemplos}

Hemos dicho que las ecuaciones de Euler-Lagrange 2.3 puedan formularse de una manera más geométrica de la forma (2.11)

$$
\frac{d^{2} q^{i}}{d t^{2}}+\Gamma_{j k}^{i} \frac{d q^{j}}{d t} \frac{d q^{k}}{d t}=-a^{i l} \frac{\partial V}{\partial q^{l}}
$$

con $\Gamma_{j k}^{i}$ los símbolos de Christoffel de la métrica $a_{i j}$, siendo $a^{i l} \frac{\partial V}{\partial q^{l}}$ las componentes contravariantes del gradiente del potencial $V$

Grosso modo la expresión anterior puede resumirse como

Términos cinemáticos (descriptivos) = Términos dinámicos (físicos/reales)

Observamos como en la parte descriptiva de la igualdad, los Símbolos de Christoffel corrigen los términos de la aceleración newtoniana incorporando de esa manera los efectos del uso de sistemas de referencia no inerciales. Cuando estos términos se trasponen al segundo miembro con intención de que la ecuación adopte la forma de ecuaciones de Newton, a las fuerzas físicas se les añade una 
corrección, las bien conocidas fuerzas ficticias.

El término de aceleración $\frac{d^{2} q^{i}}{d t^{2}}$ no es un tensor objetivo para una transformación arbitraria de coordenadas (no se transforma según las reglas de transformación tensorial) de ahí que haya de corregirlo adecuadamente.

El motivo de dicha necesidad es claro, al no estar las ecuaciones de Newton expresadas en forma tensorial, no son forminvariantes ante cualquier transformación que no sea de Galileo, de ahí que ante una transformación a sistemas no inerciales el hecho físico se manifieste de forma diferente a como Newton predice.

De esta forma, se observa claramente como la formulación lagrangiana del problema físico mejora la formulación newtoniana en cuanto que no depende del sistema de referencia utilizado. El uso de sistemas de referencia no inerciales genera de forma natural la aparición de símbolos de Christoffel no nulos.

De ahí que desde esta formulación la presencia artificial de fuerzas ficticias sea innecesaria y la expresión (3.39) sea forminvariante.

\subsubsection{Ejemplo 1}

Para la métrica euclídea en coordenadas cartesianas, esto es, con tensor métrico representado por la matriz identidad $\delta_{j}^{i}$ entonces $\Gamma_{j k}^{i}=0$ y las ecuaciones anteriores no son más que las ecuaciones de Newton

$$
\ddot{q}^{i}=-a^{i l} \frac{\partial V}{\partial q^{l}}
$$

\subsubsection{Ejemplo 2}

Para la métrica euclídea en coordenadas polares, esto es, con tensor métrico representado por la matriz $\left(a_{i j}\right)=\left(\begin{array}{cc}1 & 0 \\ 0 & r^{2}\end{array}\right)$ entonces $\Gamma_{\theta \theta}^{r}=-r, \Gamma_{r \theta}^{\theta}=$ $\Gamma_{\theta r}^{\theta}=\frac{1}{r}$ y las ecuaciones anteriores son de nuevo las ecuaciones de Newton en coordenadas polares para una partícula de masa unidad

$$
\begin{aligned}
& \frac{d^{2} r}{d t^{2}}-r\left(\frac{d \theta}{d t}\right)^{2}=-\frac{\partial V}{\partial r}=F_{r}, \\
& \frac{d^{2} \theta}{d t^{2}}+\frac{2}{r}\left(\frac{d \theta}{d t}\right)\left(\frac{d r}{d t}\right)=-r^{-2} \frac{\partial V}{\partial r}=\frac{F_{\theta}}{r} .
\end{aligned}
$$

En ambos casos puede comprobarse que la curvatura escalar de la variedad di- 
ferenciable determinada por la métrica $\left(a_{i j}\right)$ es nula, esto es, $R=0$ por lo que ambas métricas hacen referencia a una variedad diferenciable plana escrita, en cada caso, en diferentes sistemas coordenados.

El hecho de que en el ejemplo 2 los símbolos de Christoffel sean diferentes de cero únicamente indica el uso de un sistema coordenado móvil.

Toda experiencia física se lleva a cabo sobre una estructura espacio-temporal, dicho background geométrico puede estudiarse anulando el campo físico.

Así en los casos anteriores si anulamos el potencial $(V=0)$ obtendremos las ecuaciones de las trayectorias de una partícula libre en el espacio vacío, o en su equivalente geométrico, las geodésicas del espacio

$$
\begin{aligned}
& \frac{d^{2} r}{d t^{2}}-r\left(\frac{d \theta}{d t}\right)^{2}=0, \\
& r \frac{d^{2} \theta}{d t^{2}}+2\left(\frac{d \theta}{d t}\right)\left(\frac{d r}{d t}\right)=0 .
\end{aligned}
$$

\subsubsection{Ejemplo 3} como

El lagrangiano para el potencial central en coordenadas polares se define $L=T-V=\frac{1}{2} m\left[\left(\frac{d r}{d t}\right)^{2}+r^{2}\left(\frac{d \theta}{d t}\right)^{2}\right]-V(r)=\frac{1}{2} m a_{i j}(r, \theta) \frac{d x^{i}}{d t} \frac{d x^{j}}{d t}-V(r)$.

La matriz $a_{i j}(\mathbf{q})$ y su inversa $a^{i j}(\mathbf{q})\left(a_{i j} a^{j k}=\delta_{i}^{k}\right)$ serán

$$
a_{i j}=\left(\begin{array}{cc}
1 & 0 \\
0 & r^{2}
\end{array}\right), \quad a^{i j}=\left(\begin{array}{cc}
1 & 0 \\
0 & \frac{1}{r^{2}}
\end{array}\right)
$$

Por tanto la función hamiltoniana tendrá la forma

$$
H=T+V=\frac{1}{2} m\left[p_{r}^{2}+\frac{1}{r^{2}} p_{\theta}^{2}\right]+V(r)=\frac{1}{2} m a^{i j}(r, \theta) p_{i} p_{j}+V(r)
$$




\subsubsection{Versión geometrodinámica}

Las trayectorias solución de las ecuaciones de Hamilton de esta función hamiltoniana $H$ o a las ecuaciones de Euler-Lagrange para la función lagrangiana $L$ son las geodésicas de la variedad mecánica $\left(M_{E}, g_{i j}\right)$, esto es, aquella variedad Riemanniana dotada con la métrica Jacobi

$$
g_{i j}=2(E-V(r)) a_{i j}(r, \theta)=2(E-V(r))\left(\begin{array}{cc}
1 & 0 \\
0 & r^{2}
\end{array}\right) .
$$

Calculamos los símbolos de Christoffel

$$
\begin{aligned}
& \Gamma_{11}^{1}=\frac{V^{\prime}(r)}{-2 E+2 V(r)}, \\
& \Gamma_{22}^{1}=\frac{r\left(-2 E+2 V(r)+r V^{\prime}(r)\right)}{2(E-V(r))}, \\
& \Gamma_{12}^{2}=\frac{2 E-2 V(r)-r V^{\prime}(r)}{2 E r-2 r V(r)}=\Gamma_{21}^{2} .
\end{aligned}
$$

Las ecuaciones de las geodésicas (trayectorias) pueden calcularse, en general, como

$$
\frac{d^{2} x^{i}}{d s^{2}}+\Gamma_{j k}^{i} \frac{d x^{j}}{d s} \frac{d x^{k}}{d s}=0, \quad \text { con } s \equiv \text { longitud de arco. }
$$

En nuestro caso $x^{1}=r$ and $x^{2}=\theta$ por tanto las ecuaciones serán

$$
\begin{aligned}
\frac{d^{2} r}{d s^{2}}+\Gamma_{11}^{1}\left(\frac{d r}{d s}\right)^{2}+\Gamma_{22}^{1}\left(\frac{d \theta}{d s}\right)^{2} & =0 \\
\frac{d^{2} \theta}{d s^{2}}+2 \Gamma_{12}^{2} \frac{d r}{d s} \frac{d \theta}{d s} & =0
\end{aligned}
$$

Sustituyendo

$$
\begin{aligned}
\frac{d^{2} r}{d s^{2}}+\left(\frac{V^{\prime}(r)}{-2 E+2 V(r)}\right)\left(\frac{d r}{d s}\right)^{2}+\frac{r\left(-2 E+2 V(r)+r V^{\prime}(r)\right)}{2(E-V(r))}\left(\frac{d \theta}{d s}\right)^{2} & =0 \\
\frac{d^{2} \theta}{d s^{2}}+2\left(\frac{2 E-2 V(r)-r V^{\prime}(r)}{2 E r-2 r V(r)}\right) \frac{d r}{d s} \frac{d \theta}{d s} & =0 .
\end{aligned}
$$

Si queremos ver la evolución de las trayectorias en el tiempo debemos transformar el parámetro longitud de arco (s) a parámetro tiempo físico ( $t$ ) mediante el cambio $d s=2(E-V(r)) d t$ obteniendo así las bien conocidas ecuaciones 
de Newton para potenciales radiales, esto es,

$$
\begin{aligned}
& \frac{d^{2} r}{d t^{2}}-r\left(\frac{d \theta}{d t}\right)^{2}=\underbrace{-V^{\prime}(r)}_{F_{r}} \\
& r \frac{d^{2} \theta}{d t^{2}}+2 \frac{d r}{d t} \frac{d \theta}{d t}=0 .
\end{aligned}
$$




\section{Capítulo 4}

\section{Estabilidad y Caos}

Un gran cantidad de trabajo se ha publicado tratando muchos aspectos del caos en sistemas hamiltonianos. La búsqueda de indicadores de caos para trayectorias que sean eficientes como herramientas para analizar la estructura del espacio de fases asociado es una tarea muy interesante en este contexto.

Con este propósito muchos indicadores han sido propuestos en la literatura. La mayoría de ellos, como el máximo exponente de Lyapunov Skkokos, 2010, el Small Alignment Index (SALI) [Skokos et al., 2004, Dai et Field, 1995], Fast Lyapunov indicator (FLI) Lega, 2003, Barrio, 2005], Mean Exponential Growth factor of Nearby Orbits (MEGNO) Cincotta et Simó, 2000, Cincotta et al., 2003, o el analysis del mapa de frecuencias Dumas et Laskar, 1993, Losada et al., 1998, están definidos sobre el espacio de fases.

Otra alternativa expuesta en las secciones anteriores es el uso del formalismo geometrodinámico, definido en el espacio de configuración. Como hemos comentado previamente las trayectorias son identificadas con geodésicas de una cierta variedad Riemanniana llamada variedad mecánica.

Diferentes métricas pueden ser usadas en este formalismo, por ejemplo la métrica de Eisenhart [Eisenhart, 1929], Finsler [Cipriani et Bari, 1998a, y la métrica de Jacobi Casetti et al., 1996, Bari et Cipriani, 1998, Casetti et al., 2000, Cerruti-Sola et Pettini, 1995, Cerruti-Sola et Pettini, 1996

Di Cairano et al., 2019] u otras basadas principalmente en transformaciones conformes Horwitz et al., 2007

Zion et Horwitz, 2008.

En este dominio, la inestabilidad y el caos pueden ser analizados estudiando in- 
dicadores derivados de la ecuación de Jacobi-Levi-Civita (JLC) do Carmo, 1992 que describe la evolución del vector de desviación geodésico.

En esta tesis construiremos un nuevo indicador de estabilidad utilizando dicha ecuación JLC al que denominaremos SGI (stability geometric indicator) propuesto por nosotros en las Refs. Vergel et al., 2014a, Vergel et al., 2014b.

En este contexto, el caos para algunos sistemas tiene como origen la curvatura negativa de la frontera de la región de Hill |Vergel et al., 2014a, Saa, 2004, Joy et Sabir, 1993, o por la curvatura generada por la matriz de masa do Carmo, 1976, entre otras razones.

En cambio, parece haber un consenso general en relación a que el origen del caos depende de la dinámica, y puede detectarse por la fluctuación de algunos observables geométricos adecuados, como la curvatura escalar de la variedad a lo largo de geodésicas de referencia |Vergel et al., 2014a, Vergel et al., 2014b].

De hecho, la evolución del vector de desviación geodésico gobernado por las ecuaciones JLC en sistemas dos dimensionales se describe por la ecuación del oscilador armónico donde la frecuencia se reemplaza por la curvatura escalar, produciendo en ocasiones resonancia paramétrica, similarmente a lo sucedido, por ejemplo, en la ecuación de Mathieu, que ocasiona que las trayectorias se comporten de forma caótica.

Antes de hacer el estudio geometrodinámico del caos hablaremos acerca de los indicadores más conocidos definidos principalmente en el espacio de fases.

\subsection{Indicadores clásicos}

Una característica principal al régimen caótico es la extrema sensibilidad a las condiciones iniciales, esto es, trayectorias con condiciones próximas tienen evoluciones muy diferentes.

Esta idea va ligada a la de separación exponencial de trayectoria, que puede caracterizarse con los exponentes de Liapunov y al de pérdida de información relacionado con la entropía de Kolmogorov.

Otra característica propia del caos es la idea de mezcla, esto es, las órbitas se acercarán y separarán de forma impredecible. De hecho la órbita visitará a casi todos los puntos del espacio de fases. 
Definición Un sistema dinámico se dice caótico si verifica las siguientes propiedades:

1. Sensibilidad a las condiciones iniciales

2. Tiene propiedad de mezcla.

3. Las órbitas o trayectorias periódicas del sistema dinámico son densas en el espacio de fases.

Los métodos de estudio utilizados frecuentemente para el estudio de la estabilidad de los sistemas hamiltonianos son:

a) Mapa de Poincaré (dimensión 2).

b) Exponentes de Lyapunov.

c) Entropía de Kolmogorov.

d) Espectro de frecuencia. Transformada de Fourier.

Igualmente el mapa de Poincaré junto con el análisis de frecuencia nos puede dar información acerca de la estructura del espacio de fases.

\subsubsection{Mapa y Sección de Poincaré}

Una sección de Poincaré es un espacio de dimensión inferior a la del espacio de fases que debe ser transversal al flujo del sistema. En otras palabras, las trayectorias solución de las ecuaciones de movimiento deben atravesar esta sección de tal manera que aquellass trayectorias que comienzan en la sección no deben evolucionar de forma paralela a la misma con la intención de que atraviese frecuentemente dicha sección y poder obtener información dinámica interesante. Las intersecciones de la trayectoria con la sección se realizarán en instantes de tiempo diferentes.

Podemos definir mapa de Poincaré como un sistema dinámico discreto para una sucesión de instantes de tiempo $\left(t_{0}, t_{1}, \ldots\right)$ de tal manera que en cada instante obtendremos los valores $\left(q_{i}, p_{i}\right)=\left(q\left(t_{i}\right), p\left(t_{i}\right)\right)$ pudiendo definir el mapeo de un espacio de dimensión inferior a la del espacio de fases en sí mismo equivalente a las ecuaciones de movimiento en forma diferencial como

$$
\left(q_{n+1}, p_{n+1}\right)=T\left(q_{n}, p_{n}\right) \Rightarrow\left(q_{n+1}, p_{n+1}\right)=T^{n}\left(q_{0}, p_{0}\right)
$$


obteniendo una sucesión discreta de puntos:

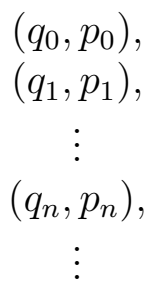

Dichas intersecciones entre trayectoria del sistema y sección de Poincaré definirá una aplicación de Poincaré que lleva un punto de la sección de Poincaré a otro de la misma sección resultado de dicha intersección sucesivamente.

Este método suele utilizarse en dimensión 2.

Suele emplearse para analizar las características del sistema original, por ejemplo:

- Una trayectoria periódica dentro de un toro invariante se convierte en una cantidad discreta y finita de puntos en la sección.

- Una trayectoria cuasi-periódica en un toro invariante se representa como una curva cerrada.

- Una trayectoria caótica se representará como una nube de puntos distribuidos aleatoriamente por la sección.

Es importante estudiar dónde se situará la sección de Poincaré ya que debe de ser un lugar muy frecuentado por las trayectorias de interés para que las intersecciones con ellas sean frecuentes y capture el máximo de información.

\subsubsection{Exponente de Lyapunov}

El exponente de Lyapunov miden la sensibilidad extrema a las condiciones iniciales calculando la tasa de divergencia exponencial de órbitas que inicialmente estaban próximas.

Para definirlo podemos pensar en un sistema discreto de una dimensión, tal que

$$
x_{n+1}=f\left(x_{n}\right)=f\left(f\left(x_{n-1}\right)\right)=f^{n}\left(x_{0}\right)
$$


siendo $x_{0}$ la condición inicial.

Imaginemos dos puntos próximos $x_{0}$ y $x_{0}+\epsilon$ que tomamos como condiciones iniciales para dos trayectorias de nuestro sistema.

Tras $n$ iteraciones, se habrán transformado en $f^{n}\left(x_{0}\right)$ y $f^{n}\left(x_{0}+\epsilon\right)$ respectivamente.

Si expresamos la separación de dichas órbitas en forma exponencial, tendremos

$$
\left\|f^{n}\left(x_{0}+\epsilon\right)-f^{n}\left(x_{0}\right)\right\|=\epsilon e^{n \lambda\left(x_{0}\right)}
$$

El exponente de Lyapunov viene dado por el parámetro $\lambda\left(x_{0}\right)$ en el límite en el que dos condiciones iniciales infinitamente próximas $(\epsilon \rightarrow 0)$ se iteran un tiempo infinito $(n \rightarrow \infty)$.

$$
\begin{gathered}
\lambda\left(x_{0}\right)=\lim _{n \rightarrow \infty} \lim _{\epsilon \rightarrow 0} \frac{1}{n} \log \left|\frac{f^{n}\left(x_{0}+\epsilon\right)-f^{n}\left(x_{0}\right)}{\epsilon}\right|= \\
\lim _{n \rightarrow} \frac{1}{n} \log \left|\frac{d f^{n}\left(x_{0}\right)}{d x_{0}}\right|,
\end{gathered}
$$

siendo $\frac{d f^{n}\left(x_{0}\right)}{d x_{0}}$ la derivada de $f^{n}(x)$ en $x=x_{0}$. Como puede deducirse de 4.3, el exponente de Lyapunov depende del punto $x_{0}$.

Aplicando la regla de la cadena a la derivada de la n-ésima iteración de f obtenemos

$$
\begin{gathered}
\lambda\left(x_{0}\right)=\lim _{n \rightarrow \infty} \frac{1}{n} \log \left|\prod_{i=0}^{n-1} f^{\prime}\left(x_{i}\right)\right|= \\
\lim _{n \rightarrow \infty} \frac{1}{n} \sum_{i=0}^{n-1} \log \left|f^{\prime}\left(x_{i}\right)\right| .
\end{gathered}
$$

Que $\lambda\left(x_{0}\right)>0$ es condición necesaria para que la trayectoria de condición inicial $x_{0}$ sea caótica. En los casos en los que el movimiento esté acotado en el espacio de fases esta condición es además suficiente, pues las trayectorias se mezclarán de forma impredecible.

Cuando la dimensión es mayor que uno tenemos que los puntos de las órbitas o trayectoria tienen la forma vectorial $\boldsymbol{x}_{n+1}=\boldsymbol{f}\left(\boldsymbol{x}_{\boldsymbol{n}}\right)$ con $\boldsymbol{x}_{\boldsymbol{n}}=\left(\boldsymbol{x}_{\boldsymbol{n}}^{\mathbf{1}}, \boldsymbol{x}_{\boldsymbol{n}}^{\mathbf{2}}, \ldots, \boldsymbol{x}_{\boldsymbol{n}}^{\boldsymbol{N}}\right)$, teniendo entonces $N$ exponentes de Lyapunov.

Si conociéramos la función $f$, el jacobiano de la función vectorial $\boldsymbol{f}$ tiene la forma 


$$
D f^{n+1}\left(\boldsymbol{x}_{\boldsymbol{n}}\right)=\left(\begin{array}{cccc}
\frac{\partial f^{1}}{\partial x^{1}}\left(\boldsymbol{x}_{\boldsymbol{n}}\right), & \frac{\partial f^{1}}{\partial x^{2}}\left(\boldsymbol{x}_{\boldsymbol{n}}\right) & \ldots & \frac{\partial f^{1}}{\partial x^{n}}\left(\boldsymbol{x}_{\boldsymbol{n}}\right) \\
\frac{\partial f^{2}}{\partial x^{1}}\left(\boldsymbol{x}_{\boldsymbol{n}}\right), & \frac{\partial f^{2}}{\partial x^{2}}\left(\boldsymbol{x}_{\boldsymbol{n}}\right) & \ldots & \frac{\partial f^{2}}{\partial x^{n}}\left(\boldsymbol{x}_{\boldsymbol{n}}\right) \\
\vdots & \vdots & \vdots & \vdots \\
\frac{\partial f^{N}}{\partial x^{1}}\left(\boldsymbol{x}_{\boldsymbol{n}}\right), & \frac{\partial f^{N}}{\partial x^{2}}\left(\boldsymbol{x}_{\boldsymbol{n}}\right) & \ldots & \frac{\partial f^{N}}{\partial x^{n}}\left(\boldsymbol{x}_{\boldsymbol{n}}\right)
\end{array}\right)
$$

Definimos el primer exponente de Lyapunov como

$$
\lambda_{1}\left(x_{0}\right)=\lim _{n \rightarrow \infty} \frac{1}{n} \log \left|D f^{n}\left(x_{0}\right)\right| .
$$

En general si el sistema dinámico es $N$-dimensional, tendrá $N$ exponentes de Lyapunov $\lambda_{1} \geq \lambda_{2} \geq \cdots \geq \lambda_{N}$.

Por ejemplo para $N=3$

- $e^{\lambda_{1}\left(\boldsymbol{x}_{0}\right)}$ es el máximo factor que amplifica el error $\epsilon$.

- $e^{\lambda_{1}\left(\boldsymbol{x}_{\mathbf{0}}\right)+\lambda_{2}\left(\boldsymbol{x}_{\mathbf{0}}\right)}$ es el máximo factor que amplifica el área.

- $e^{\lambda_{1}\left(\boldsymbol{x}_{\mathbf{0}}\right)+\lambda_{2}\left(\boldsymbol{x}_{\mathbf{0}}\right)+\lambda_{3}\left(\boldsymbol{x}_{\mathbf{0}}\right)}$ es el máximo factor que amplifica el volumen.

En general no se conoce la forma analítica de $f$, por lo que en este caso podemos calcular numéricamente de forma sencilla $\lambda_{1}\left(\boldsymbol{x}_{\mathbf{0}}\right)$ calculando la distancia entre dos trayectorias una digamos de referencia ý otra test que inicialmente estén próximas y comparando su distancia asintóticamente en el tiempo.

Para órbitas caóticas la distancia entre la órbita de referencia y la test crece excesivamente, generando un problema numérico. Para evitar este problema se suele renormalizar la separación entre órbitas periódicamente.

Cuando la distancia de separación entre órbitas supera cierta cantidad establecida de antemano (threshold), normalizamos el vector separación manteniendo la orientación relativa entre las dos órbitas.

Si definimos $\boldsymbol{d}_{n}=\boldsymbol{f}^{n}\left(\boldsymbol{x}_{\mathbf{0}}+\boldsymbol{\epsilon}\right)-\boldsymbol{f}^{n}\left(\boldsymbol{x}_{\mathbf{0}}\right)$ el vector de separación entre órbitas inicialmente próximas.

Supongamos que hemos fijado el threshold en $D$ y que en la iteración $n_{1}$ se 
cumple $\left|\boldsymbol{d}_{n 1}\right|>D$ entonces renormalizamos el vector separación $\boldsymbol{d}_{n}$

$$
\begin{gathered}
\boldsymbol{d}_{n}=\boldsymbol{f}^{n}\left(\boldsymbol{x}_{\mathbf{0}}\right)+\frac{\boldsymbol{f}^{n}\left(\boldsymbol{x}_{\mathbf{0}}+\boldsymbol{\epsilon}\right)-\boldsymbol{f}^{n}\left(\boldsymbol{x}_{\mathbf{0}}\right)}{\left|\boldsymbol{d}_{n}\right|} \cdot \epsilon= \\
\boldsymbol{f}^{n}\left(\boldsymbol{x}_{\mathbf{0}}\right)+\frac{\boldsymbol{f}^{n}\left(\boldsymbol{x}_{\mathbf{0}}+\boldsymbol{\epsilon}\right)-\boldsymbol{f}^{n}\left(\boldsymbol{x}_{\mathbf{0}}\right)}{\left|\boldsymbol{f}^{n}\left(\boldsymbol{x}_{\mathbf{0}}+\boldsymbol{\epsilon}\right)-\boldsymbol{f}^{n}\right|} \cdot \epsilon
\end{gathered}
$$

Nuestro objetivo será calcular

$$
\lambda(t)=\frac{1}{t-t_{0}} \log \left(\frac{d(t)}{d_{0}}\right)
$$

donde $d(t)=\left|\boldsymbol{d}_{t}\right|$ Para concretar el cálculo del exponente de Lyapunov en un proceso discreto de tiempos definimos la evolución de las distancias entre ambas órbitas $d_{t_{n}}$ en el momento $t_{n}$, los valores de $t_{n}$ se corresponden con los momentos en que las distancias $d(t)$ son mayores que el threshold $D$ fijado previamente, así

1. Para $t=t_{1}$ obtenemos $d_{t_{1}}=d_{t_{0}} \cdot e^{\lambda_{1} t_{1}}$ y definimos $\alpha_{1} \equiv \frac{d_{t_{1}}}{d_{t_{0}}}$.

2. Renormalizamos $d_{t_{1}}$ haciendo $d_{t_{1}}=\frac{d_{t_{1}}}{\alpha_{1}}=\frac{d_{t_{0}} \cdot e^{\lambda_{1} t_{1}}}{\alpha_{1}}$.

3. Para $t=t_{2}$ obtenemos $d_{t_{2}}=d_{t_{1}} \cdot e^{\lambda_{2}\left(t_{2}-t_{1}\right)}$ y definimos $\alpha_{2} \equiv \frac{d_{t_{2}}}{d_{t_{0}}}$, sustituyendo la expresión anterior en $d_{t_{1}}$ obtenemos $d_{t_{2}}=\left(\frac{d_{t_{0}} e^{\lambda_{1} t_{1}}}{\alpha_{1}}\right) \cdot e^{\lambda_{2}\left(t_{2}-t_{1}\right)}=\frac{d_{t_{0}}}{\alpha_{1}} e^{\lambda_{2} t_{2}}$.

4. En la última igualdad hemos utilizado la aproximación $\lambda_{1} \approx \lambda_{2}$ por ser los intervalos de tiempo $t_{n}$ pequeños.

5. Renormalizamos $d_{t_{2}}$ haciendo $d_{t_{2}}=\frac{d_{t_{2}}}{\alpha_{2}}=\frac{d_{t_{0}} \cdot e^{\lambda_{1} t_{1}}}{\alpha_{1} \cdot \alpha_{2}}$.

6. Para $t=t_{3}$ obtenemos $d_{t_{3}}=d_{t_{2}} \cdot e^{\lambda_{3}\left(t_{3}-t_{2}\right)}$ y definimos $\alpha_{3} \equiv \frac{d_{t_{3}}}{d_{t_{0}}}$.

Sustituyendo la expresión anterior en $d_{t_{2}}$ obtenemos

$$
\begin{aligned}
& d_{t_{3}}=\left(\frac{d_{t_{0}} e^{\lambda_{2} t_{2}}}{\alpha_{1} \alpha_{2}}\right) \cdot e^{\lambda_{3}\left(t_{3}-t_{2}\right)}=\frac{d_{t_{0}}}{\alpha_{1} \cdot \alpha_{2}} e^{\lambda_{3} t_{3}} \Rightarrow \\
& d_{t_{3}}=\frac{d_{t_{0}}}{\alpha_{1} \cdot \alpha 2} e^{\lambda_{3} t_{3}} \Rightarrow \frac{d_{t_{3}}}{d_{t_{0}}}=\frac{1}{\alpha_{1} \cdot \alpha_{2}} e^{\lambda_{3} t_{3}} \Rightarrow \\
& \alpha_{1} \cdot \alpha_{2} \cdot \alpha_{3}=e^{\lambda_{3} t_{3}} \Rightarrow \lambda_{3}=\frac{1}{t_{3}-t_{0}} \ln \left(\alpha_{1} \cdot \alpha_{2} \cdot \alpha_{3}\right)=\frac{1}{t_{3}-t_{0}} \sum_{i=1}^{3} \ln \alpha_{i} .
\end{aligned}
$$


Entonces para $t=t_{n}$ con $t_{0}=0$ obtenemos la expresión

$$
\lambda_{n}=\frac{1}{t_{n}} \sum_{i=1}^{n} \log \alpha_{i},
$$

$\operatorname{con} \alpha_{i}=\frac{d_{t_{i}}}{d_{0}}$.

\subsubsection{Espectro de frecuencias. Transformada de Fou- rier}

La representación espectral de series temporales o de un proceso nos permite mostrar las componentes periódicas.

La idea es expresar el proceso (representado a través de una serie temporal) como combinaciones lineales de funciones trigonométricas o exponenciales complejas e identificar las frecuencias más relevantes para la evolución de nuestro sistema.

En un sistema hamiltoniano una trayectoria se puede representar por una resolución numérica discreta, digamos $x(0), x(\Delta t), x(2 \Delta t), \ldots, x(n \Delta t)$. En este caso utilizaremos la Transformada dicreta de Fourier. Así, sea la secuencia $x_{1}, x_{2}, \ldots, x_{n}$ su transformada de Fourier será la secuencia $\hat{x}_{1}, \hat{x}_{2}, \ldots, \hat{x}_{n}$ definida como

$$
\hat{x}_{k}=f\left(\frac{k}{n \Delta t}\right)=\frac{1}{\sqrt{n \Delta t}} \sum_{j=1}^{n} x_{j} \cdot e^{-i \frac{2 \pi j k}{n \Delta t}} .
$$

Dada la serie temporal finita $x_{1}, x_{2}, \ldots, x_{T}$ puede escribirse como combinación lineal de funciones trigonométricas de la siguiente forma

$$
\hat{x}_{k}=\sum_{k=0}^{n}\left(a_{k} \cdot \cos \omega_{k} t+b_{k} \cdot \sin \omega_{k} t\right),
$$

donde $\omega_{k}=\frac{2 \pi k}{T}$ son las naturales, y $a_{k}$ y $b_{k}$ las amplitudes de las funciones trigonométricas.

Los coeficientes $a_{k}$ y $b_{k}$ se pueden obtener como

$$
a_{k}=\frac{2}{T} \sum_{t=1}^{T} x_{t} \cdot \cos \omega_{k} t, \quad b_{k}=\frac{2}{T} \sum_{t=1}^{T} x_{t} \cdot \sin \omega_{k} t
$$


Así la serie temporal se puede representar de dos maneras diferentes, como conjunto de pares, bien sea en el dominio de tiempo $\left(t, x_{t}\right)$ o en el dominio de frecuencias $\left(\omega_{k},\left|\hat{x}_{k}\right|\right)$, donde $E_{k}=\left|\hat{x}_{k}\right|=a_{k}^{2}+b_{k}^{2}$ la llamamos energía de la frecuencia $\omega_{k}$.

Si la señal es periódica de periodo $T$ se obtiene un solo valor de $E_{k}$, si son funciones sinusoidales correspondientes a su frecuencia $\nu=\frac{2 \pi}{T}$ o un número finito de valores de $E_{k}$ correspondientes a sus múltiplos $\nu, 2 \nu, 3 \nu, \ldots$ en el caso de funciones no sinusoidales.

Si la señal es cuasiperiódica, esto es, una función de $N$ frecuencias $\nu_{k}$, el espectro de Fourier debe incluir contribuciones de todas ellas, aunque sólo sean relevantes unas pocas.

Si la señal es caótica, su espectro es continuo. En este último caso, no hay máximos definidos que muestren las frecuencias características.

\subsection{Ecuación JLC e indicador SGI}

La curvatura seccional $K(p, \sigma)$ en un punto $p \in M$ y plano tangente $\sigma \subset T_{p} M$ en la variedad Riemanniana $M$ en ese punto determina el ritmo de dispersión de geodésicas comenzando en el punto $p$ siendo inicialmente tangentes a $M$. ( $K$ se corresponde con la curvatura gaussiana en el caso bidimensional).

La evolución de esta desviación geodésica, $\mathbf{J}(s)$, a lo largo de la trayectoria de referencia $\gamma(s)$ viene descrita por la ecuación de Jacobi-Levi-Civita (JLC).

$$
\frac{D^{2} \mathbf{J}}{d s^{2}}+R[\dot{\gamma}(s), \mathbf{J}(s)] \dot{\gamma}(s)=0,
$$

en coordenadas locales $\frac{D^{2} J^{i}}{d s^{2}}=-R_{j k l}^{i} \dot{\gamma}^{j} \dot{\gamma}^{l} J^{k}=-S_{j}^{i} J^{j}$ donde $S_{j}^{i}$ también se denomina tensor de estabilidad y con $\gamma: s \in[0, a] \rightarrow M$ geodésica en $M$, $\dot{\gamma}(s)=d \mathbf{q} / d s$, y siendo $R(\mathbf{X}, \mathbf{Y}): T_{p} M \longrightarrow T_{p} M$ dado por $R(X, Y) Z=$ $\nabla_{X} \nabla_{Y} Z-\nabla_{Y} \nabla_{X} Z+\nabla_{[X, Y]} Z$ con $X, Y, Z \in T_{p} M$ el llamado tensor de curvatura de Riemann-Christoffel.

En una carta local podemos escribir $R\left(\mathbf{e}_{i}, \mathbf{e}_{j}\right) \mathbf{e}_{k}=R_{i j k}^{l} \mathbf{e}_{l}$ donde $R_{i j k}^{l}=\Gamma_{i k}^{s} \Gamma_{j s}^{l}-$ $\Gamma_{j k}^{s} \Gamma_{i s}^{l}+\frac{\partial}{\partial x_{j}} \Gamma_{i k}^{l}-\frac{\partial}{\partial x_{i}} \Gamma_{j k}^{l}$. 


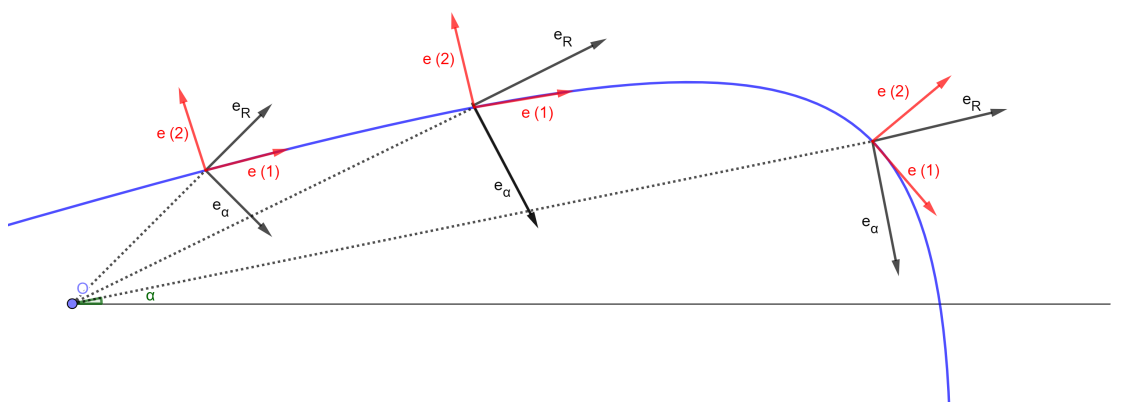

Figura 4.1: Base canónica (negro) y base transportada paralelamente (rojo) en tres puntos de una geodésica (azul).

La ecuación (4.13) puede ser expresada de una forma conveniente utilizando un sistema de referencia móvil formado por el conjunto de vectores ortonormales $\left\{\mathbf{e}_{(1)}, \mathbf{e}_{(2)}\right\}$ transportados paralelamente a lo largo de la trayectoria $\gamma(s)$

$$
\mathbf{e}_{(i)}=\mathbf{e}_{(i)}^{j} \mathbf{e}_{j}, \quad i=1,2,
$$

donde $\nabla \mathbf{e}_{(i)} / d s \equiv D \mathbf{e}_{(i)} / d s=0$, en coordenadas locales equivalente a

$$
\left(\frac{d \mathbf{e}_{(i)}^{\alpha}}{d t}+\mathbf{e}_{(i)}^{\mu} \Gamma_{\mu \beta}^{\alpha} \frac{d q^{\beta}}{d t}\right) \mathbf{e}_{\alpha}=0
$$

Las componentes $\mathbf{e}_{(i)}^{\alpha}$ de los vectores transportados paralelamente $\mathbf{e}_{(i)}$ pueden ser calculadas utilizando el Teorema de Taylor,

$$
\left.\mathbf{e}_{(i)}^{\alpha}\right|_{t}=\left.\mathbf{e}_{(i)}^{\alpha}\right|_{t_{0}}-\left.\mathbf{e}_{(i)}^{\mu} \Gamma_{\mu \beta}^{\alpha} \frac{d q^{\beta}}{d t}\right|_{t_{0}}\left(t-t_{0}\right)+o\left(t-t_{0}\right)^{2}
$$

fijando el vector tangente a la trayectoria de referencia, $\mathbf{e}_{(1)}(s)=\dot{\gamma}(s)$, como primer vector de la base móvil,

Se ilustra la construcción de la base paralelamente transportada en la figura 4.1

$$
g_{i j}(\mathbf{q}) \mathbf{e}_{(a)}^{i} \mathbf{e}_{(b)}^{j}=\delta_{a b}
$$

Así, en cualquier punto de la trayectoria de referencia se obtiene el nuevo sistema de referencia en términos de las coordenadas en la carta local para $M$

$$
\begin{aligned}
& \mathbf{e}_{(1)}(s)=\dot{\gamma}^{1}(s) \mathbf{e}_{1}(s)+\dot{\gamma}^{2}(s) \mathbf{e}_{2}(s), \\
& \mathbf{e}_{(2)}(s)=\sqrt{a_{22} / a_{11}} \dot{\gamma}^{2} \mathbf{e}_{1}(s)-\sqrt{a_{22} / a_{11}} \dot{\gamma}^{1} \mathbf{e}_{2}(s) .
\end{aligned}
$$




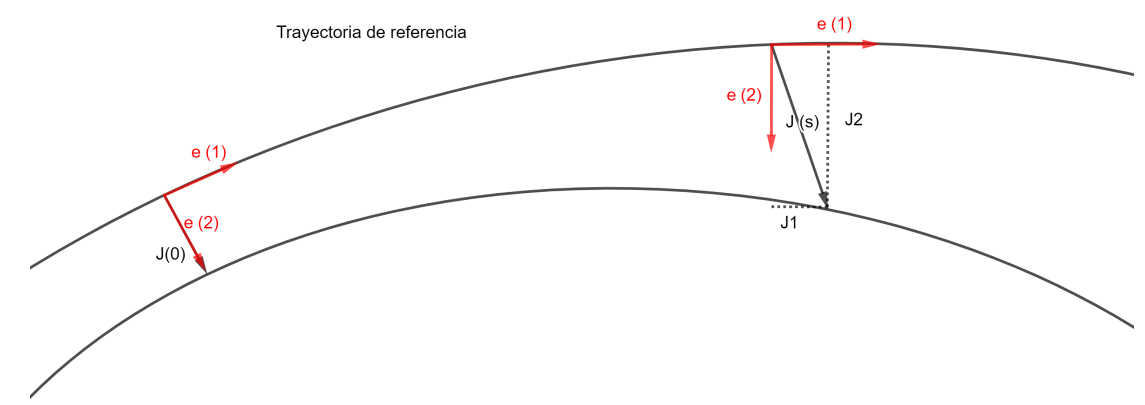

Figura 4.2: Evolución del vector de separación geodésico $\mathbf{J}$ a lo largo de una geodésica.

Observamos que estas últimas condiciones sólo dependen del vector tangente y del punto en cuestión; esto no será igual en mayores dimensiones.

En el nuevo sistema de referencia, $\left\{\mathbf{e}_{(1)}, \mathbf{e}_{(2)}\right\}$, el campo vectorial $\mathbf{J}(s)$ se expresa como $\mathbf{J}(s)=J^{i}(s) \mathbf{e}_{(i)}(s), i=1,2$ (ver figura 4.2 y entonces 4.13) se transforma en

$$
\left(\begin{array}{c}
\frac{d^{2} J^{1}}{d s^{2}} \\
\frac{d^{2} J^{2}}{d s^{2}}
\end{array}\right)+\left(\begin{array}{cc}
\left\langle R\left(\mathbf{v}, \mathbf{e}_{(1)}\right) \mathbf{v}, \mathbf{e}_{(1)}\right\rangle & \left\langle R\left(\mathbf{v}, \mathbf{e}_{(2)}\right) \mathbf{v}, \mathbf{e}_{(1)}\right\rangle \\
\left\langle R\left(\mathbf{v}, \mathbf{e}_{(1)}\right) \mathbf{v}, \mathbf{e}_{(2)}\right\rangle & \left\langle R\left(\mathbf{v}, \mathbf{e}_{(2)}\right) \mathbf{v}, \mathbf{e}_{(2)}\right\rangle
\end{array}\right)\left(\begin{array}{c}
J^{1} \\
J^{2}
\end{array}\right)=0
$$

donde $\langle$,$\rangle es el producto escalar, y \left\langle\mathbf{e}_{i}, \mathbf{e}_{j}\right\rangle=g_{i j}$ son los coeficientes métricos (primera forma fundamental).

Ahora, si $\mathbf{v}=\mathbf{e}_{(1)}=\dot{\gamma}$ y considerando las simetrías del tensor de curvatura, es decir, $\left\langle R\left(\mathbf{e}_{i}, \mathbf{e}_{j}\right) \mathbf{e}_{k}, \mathbf{e}_{l}\right\rangle=R_{i j k l}=R_{k l i j}=-R_{j i k l}=-R_{i j l k}$, 4.19 se puede reducir a

$$
\left(\begin{array}{c}
\frac{d^{2} J^{1}}{d s^{2}} \\
\frac{d^{2} J^{2}}{d s^{2}}
\end{array}\right)+\left(\begin{array}{cc}
0 & 0 \\
0 & \left\langle R\left(\mathbf{e}_{(1)}, \mathbf{e}_{(2)}\right) \mathbf{e}_{(1)}, \mathbf{e}_{(2)}\right\rangle
\end{array}\right)\left(\begin{array}{c}
J^{1} \\
J^{2}
\end{array}\right)=0
$$

Teniendo ahora en cuenta que

$$
\left|\mathbf{e}_{(1)} \wedge \mathbf{e}_{(2)}\right|=\sqrt{\left|\mathbf{e}_{(2)}\right|\left|\mathbf{e}_{(2)}\right|-\left\langle\mathbf{e}_{(1)}, \mathbf{e}_{(2)}\right\rangle}=1,
$$

donde $\wedge$ denota el producto vectorial, podemos escribir que

$$
\begin{aligned}
\left\langle R\left(\mathbf{e}_{(1)}, \mathbf{e}_{(2)}\right) \mathbf{e}_{(1)}, \mathbf{e}_{(2)}\right\rangle & =\frac{\left\langle R\left(\mathbf{e}_{(1)}, \mathbf{e}_{(2)}\right) \mathbf{e}_{(1)}, \mathbf{e}_{(2)}\right\rangle}{\left|\mathbf{e}_{(1)} \wedge \mathbf{e}_{(2)}\right|^{2}} \\
& =K\left(\mathbf{e}_{(1)}, \mathbf{e}_{(2)}\right) \equiv K(\sigma),
\end{aligned}
$$


con $K(\sigma)$ la curvatura seccional para el plano generado por $\mathbf{e}_{(1)}$ y $\mathbf{e}_{(2)}$ en $\gamma(s)$, que utilizando (B.72) también puede ser expresado como

$$
\begin{aligned}
K(\sigma) & =K\left(\mathbf{e}_{(1)}, \mathbf{e}_{(2)}\right)=\frac{R_{l j h k} e_{(1)}^{l} e_{(1)}^{h} e_{(2)}^{j} e_{(2)}^{k}}{\left(g_{l h} g_{j k}-g_{l k} g_{j h}\right) e_{(1)}^{l} e_{(1)}^{h} e_{(2)}^{j} e_{(2)}^{k}} \\
& =\frac{1}{\left|g_{i j}\right|} R_{1212}=\frac{\hat{R}}{2}
\end{aligned}
$$

con $\hat{R}$ la curvatura escalar. Podemos escribir 4.20 como

$$
\begin{aligned}
& \frac{d^{2} J^{1}}{d s^{2}}=0, \\
& \frac{d^{2} J^{2}}{d s^{2}}=-\frac{R_{1212}}{\left|g_{i j}\right|} J^{2}=-\frac{\hat{R}(s)}{2} J^{2} .
\end{aligned}
$$

La evolución de $J^{1}$ es como mucho lineal y la estabilidad queda determinada por el escalar $J^{2}$ (referido como $J$ ) a lo largo de la trayectoria de referencia. Se puede observar que el tensor de estabilidad depende únicamente del punto y no depende de la evolución de los propios vectores de la base, cosa que no sucede en mayores dimensiones.

\subsubsection{Dimensión dos:}

Elegimos como base astuta una base ortonormal de vectores paralelamente transportados

$$
\left\{\mathbf{e}_{(1)}, \mathbf{e}_{(2)}\right\} \operatorname{con} \frac{\nabla \mathbf{e}_{(i)}}{d s} \equiv \frac{D \mathbf{e}_{(i)}}{d s}=0 \forall i
$$

La ecuación JLC tiene la forma

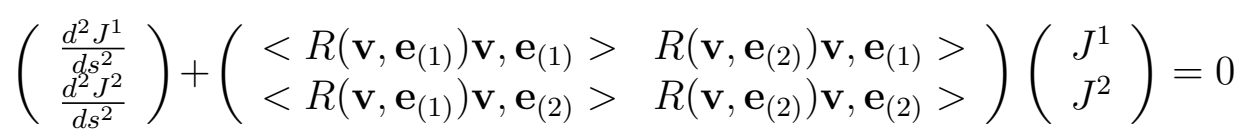

con $\mathbf{v}=\mathbf{e}_{(1)}$ then $<R\left(\mathbf{v}, \mathbf{e}_{(j)}\right) \mathbf{v}, \mathbf{e}_{(i)}>=<R\left(\mathbf{e}_{(1)}, \mathbf{e}_{(j)}\right) \mathbf{e}_{(1)}, \mathbf{e}_{(i)}>$ y aplicando las simetrías de $R_{i j k l}$

$$
\left(\begin{array}{c}
\frac{d^{2} J^{1}}{d s^{2}} \\
\frac{d^{2} J^{2}}{d s^{2}}
\end{array}\right)+\left(\begin{array}{cc}
0 & 0 \\
0 & K\left(\mathbf{e}_{(1)}, \mathbf{e}_{(2)}\right)
\end{array}\right)\left(\begin{array}{l}
J^{1} \\
J^{2}
\end{array}\right)=0
$$

expresamos la base astuta $\left\{\mathbf{e}_{(1)}, \mathbf{e}_{(2)}\right\}$ en términos de la base canónica $\left\{\mathbf{e}_{1}, \mathbf{e}_{2}\right\}$ 
como

$$
\begin{aligned}
& \mathbf{e}_{(1)}=e_{(1)}^{1} \mathbf{e}_{1}+e_{(1)}^{2} \mathbf{e}_{2} \\
& \mathbf{e}_{(2)}=e_{(2)}^{1} \mathbf{e}_{1}+e_{(2)}^{2} \mathbf{e}_{2}
\end{aligned}
$$

por tanto el vector $\mathbf{J}$ puede ser expresado como

$$
\mathbf{J}(s)=J^{i} \mathbf{e}_{(i)}(s), \quad i=1,2
$$

Aplicando la expresión ( B.72 del Apéndice expresamos la curvatura escalar como

$$
K(\sigma)=K\left(\mathbf{e}_{(1)}, \mathbf{e}_{(2)}\right)=\frac{R_{l j h k} e_{(1)}^{l} e_{(1)}^{h} e_{(2)}^{j} e_{(2)}^{k}}{\left(g_{l h} g_{j k}-g_{l k} g_{j h}\right) e_{(1)}^{l} e_{(1)}^{h} e_{(2)}^{j} e_{(2)}^{k}}=\frac{R_{l j h k} e_{(1)}^{l} e_{(1)}^{h} e_{(2)}^{j} e_{(2)}^{k}}{\left\|\mathbf{e}_{(1)} \times \mathbf{e}_{(2)}\right\|^{2}}
$$

tomando $\mathbf{v}=\mathbf{e}_{(1)}$

$$
R\left(\mathbf{e}_{(1)}, \mathbf{J}\right) \mathbf{e}_{(1)}=\sum_{j=1}^{2}<R\left(\mathbf{e}_{(1)}, J^{i} \mathbf{e}_{i}\right) \mathbf{e}_{(1)}, \mathbf{e}_{(j)}>\mathbf{e}_{(j)}
$$

$$
R\left(\mathbf{e}_{(1)}, \mathbf{J}\right) \mathbf{e}_{(1)}=J^{2}<R\left(\sum_{i=1}^{2} e_{(1)}^{i} \mathbf{e}_{i}, \sum_{j=1}^{2} e_{(2)}^{j} \mathbf{e}_{j}\right)\left(\sum_{k=1}^{2} e_{(1)}^{k} \mathbf{e}_{(k)}\right),\left(\sum_{l=1}^{2} e_{(2)}^{l} \mathbf{e}_{l}\right)>\mathbf{e}_{(2)}
$$

$$
\begin{array}{r}
R\left(\mathbf{e}_{(1)}, \mathbf{J}\right) \mathbf{e}_{(1)}= \\
J^{2}\left(\sum_{i, j, k, l=1}^{2} e_{(1)}^{i} e_{(2)}^{j} e_{(2)}^{k} e_{(2)}^{l}<R\left(\mathbf{e}_{i}, \mathbf{e}_{j}\right) \mathbf{e}_{k}, \mathbf{e}_{l}>\right) \mathbf{e}_{(2)} \\
R\left(\mathbf{e}_{(1)}, \mathbf{J}\right) \mathbf{e}_{(1)}=J^{2}\left\{\begin{array}{l}
e_{(1)}^{1} e_{(2)}^{2} e_{(1)}^{1} e_{(2)}^{2}<R\left(\mathbf{e}_{1}, \mathbf{e}_{2}\right) \mathbf{e}_{1}, \mathbf{e}_{2}>+ \\
\\
e_{(1)}^{1} e_{(2)}^{2} e_{(1)}^{2} e_{(2)}^{1}<R\left(\mathbf{e}_{1}, \mathbf{e}_{2}\right) \mathbf{e}_{2}, \mathbf{e}_{1}>+ \\
\\
e_{(1)}^{2} e_{(2)}^{1} e_{(1)}^{1} e_{(2)}^{2}<R\left(\mathbf{e}_{2}, \mathbf{e}_{1}\right) \mathbf{e}_{1}, \mathbf{e}_{2}>+ \\
\\
\left.e_{(1)}^{2} e_{(2)}^{1} e_{(1)}^{2} e_{(2)}^{1}<R\left(\mathbf{e}_{2}, \mathbf{e}_{1}\right) \mathbf{e}_{2}, \mathbf{e}_{1}>\right\} \mathbf{e}_{2}
\end{array}\right.
\end{array}
$$

$$
\begin{aligned}
R\left(\mathbf{e}_{(1)}, \mathbf{J}\right) \mathbf{e}_{(1)}=J^{2}\{ & e_{(1)}^{1} e_{(2)}^{2} e_{(1)}^{1} e_{(2)}^{2} R_{1212}- \\
& e_{(1)}^{1} e_{(2)}^{2} e_{(1)}^{2} e_{(2)}^{1} R_{1212}- \\
& e_{(1)}^{2} e_{(2)}^{1} e_{(1)}^{1} e_{(2)}^{2} R_{1212}+ \\
& \left.e_{(1)}^{2} e_{(2)}^{1} e_{(1)}^{2} e_{(2)}^{1} R_{1212}\right\} \mathbf{e}_{2}
\end{aligned}
$$




$$
\begin{gathered}
R\left(\mathbf{e}_{(1)}, \mathbf{J}\right) \mathbf{e}_{(1)}=J^{2}\left\{\left[e_{(1)}^{1} e_{(2)}^{2}\right]^{2}-2 e_{(1)}^{1} e_{(1)}^{2} e_{(2)}^{1} e_{(2)}^{2}+\left[e_{(1)}^{2} e_{(2)}^{1}\right]^{2}\right\} R_{1212} \mathbf{e}_{(2)} \\
R\left(\mathbf{e}_{(1)}, \mathbf{J}\right) \mathbf{e}_{(1)}=J^{2}\left\{\left[e_{(1)}^{1} e_{(2)}^{2}-e_{(1)}^{2} e_{(2)}^{1}\right]^{2}\right\} R_{1212} \mathbf{e}_{(2)}
\end{gathered}
$$

observando que

$$
\begin{aligned}
& 1=\left\|\mathbf{e}_{1} \times \mathbf{e}_{2}\right\|=\left(g_{l h} g_{j k}-g_{l k} g_{j h}\right) e_{(1)}^{l} e_{(1)}^{h} e_{(2)}^{j} e_{(2)}^{k}= \\
& g_{11} g_{22}\left[e_{(1)}^{1} e_{(2)}^{2}-e_{(1)}^{2} e_{(2)}^{1}\right]^{2}=\left|g_{i j}\right|\left[e_{(1)}^{1} e_{(2)}^{2}-e_{(1)}^{2} e_{(2)}^{1}\right]^{2}
\end{aligned}
$$

entonces

$$
\begin{aligned}
& R\left(\mathbf{e}_{(1)}, \mathbf{J}\right) \mathbf{e}_{(1)}=J^{2}\left\{\left[e_{(1)}^{1} e_{(2)}^{2}-e_{(1)}^{2} e_{(2)}^{1}\right]^{2}\right\} R_{1212} \mathbf{e}_{(2)}= \\
& J^{2} \frac{1}{\left|g_{i j}\right|} R_{1212} \mathbf{e}_{(2)}=J^{2} \frac{R}{2} \mathbf{e}_{(2)}
\end{aligned}
$$

con $R=$ curvatura escalar

$$
\begin{aligned}
\frac{d^{2} J^{1}}{d s^{2}} & = \\
\frac{d^{2} J^{2}}{d s^{2}} & =-\underbrace{\frac{R_{1212}}{\left|g_{i j}\right|}}_{\frac{R}{2}} J^{2}
\end{aligned}
$$

El mismo análisis se puede realizar en coordenadas locales partiendo de la expresión geométrica del tensor de curvatura, dado que se cumple

$$
R(\mathbf{v}, \mathbf{J}) \mathbf{v}=\sum_{j=1}^{2}<R(\mathbf{v}, \mathbf{J}) \mathbf{v}, \mathbf{e}_{j}>\mathbf{e}_{j}
$$

En nuestro caso, $\dot{\gamma}(s)=\frac{\mathbf{d q}}{d s}=\mathbf{v}$ y $\mathbf{J}(s)$ el campo desviación geodésica a lo largo de la trayectoria de referencia $\gamma(s)$, entonces

$$
\frac{D^{2} J^{i}}{d s^{2}}=-R_{j k l}^{i} \dot{\gamma}^{j} \dot{\gamma}^{l} J^{k}=-S_{k}^{i} J^{k}
$$


con $S^{i}{ }_{k}$ el tensor de estabilidad, en una carta local $R\left(\mathbf{e}_{i}, \mathbf{e}_{j}\right) \mathbf{e}_{k}=R^{l}{ }_{i j k} \mathbf{e}_{l} \mathrm{y}$ $R_{i j k}^{l}=\Gamma_{i k}^{s} \Gamma_{j s}^{l}-\Gamma_{j k}^{s} \Gamma_{i s}^{l}+\frac{\partial \Gamma_{i k}^{l}}{\partial q^{j}}-\frac{\partial \Gamma_{j k}^{l}}{\partial q^{i}}$

$$
\left(\begin{array}{c}
\frac{D^{2} J^{1}}{d s^{2}} \\
\frac{D^{2} J^{2}}{d s^{2}}
\end{array}\right)=-\underbrace{\left(\begin{array}{cc}
R_{212}^{1}\left(\dot{\gamma}^{2}\right)^{2} & -R_{212}^{1} \dot{\gamma}^{1} \dot{\gamma}^{2} \\
-R_{212}^{1} \dot{\gamma}^{1} \dot{\gamma}^{2} & R^{1}{ }_{212}\left(\dot{\gamma}^{1}\right)^{2}
\end{array}\right)}_{S_{k}^{i}}\left(\begin{array}{c}
J^{1} \\
J^{2}
\end{array}\right)
$$

la matriz $\left(S_{k}^{i}\right)$ es simétrica y define una aplicación autoadjunta $A_{\dot{\gamma}}$ relativa a $g_{i j}$ como $A_{\dot{\gamma}}: T_{p} M \rightarrow T_{p} M$ donde $A_{\dot{\gamma}}(\mathbf{u})=R(\dot{\gamma}, \mathbf{u}) \dot{\gamma}$, en una carta local $\dot{\gamma}=\dot{\gamma}^{i} \mathbf{e}_{i}$ y $\mathbf{u}=u^{j} \mathbf{e}_{j}$ puede ser expresado como $A_{\dot{\gamma}}(\mathbf{u})=\dot{\gamma}^{j} \dot{\gamma}^{k} u^{l} R_{j l k}^{m} \mathbf{e}_{m}$ y la forma cuadrática asociada $Q: T_{p} M \rightarrow \mathbb{R}$

$$
Q(\mathbf{u})=<A_{\dot{\gamma}}(\mathbf{u}), \mathbf{u}>=<R(\dot{\gamma}, \mathbf{u}) \dot{\gamma}, \mathbf{u}>=\underbrace{\dot{\gamma}^{j} \dot{\gamma}^{k} R_{j l k}^{m} g_{m n}}_{S_{n l}} u^{l} u^{n}
$$

con $<,>$ el producto escalar, puede ser diagonalizada resolviendo

$$
\left|S_{n l}-\lambda g_{n l}\right|=0 \quad \lambda \in \mathbb{R}
$$

con autovalores $\lambda_{1}=0$ y $\lambda_{2}=\frac{R_{1212}}{|g|}$ y los correspondientes autovectores $\mathbf{u}_{1}=\left(\frac{\dot{\gamma}^{1}}{\dot{\gamma}^{2}}, 1\right)$ y $\mathbf{u}_{2}=\left(\frac{\dot{\gamma}^{1} \dot{\gamma}^{2}}{\left(\dot{\gamma}^{2}\right)^{2}-\frac{g_{11}}{|g|}}, 1\right)$

Estos autovectores una vez normalizados definen una base adecuada $\left\{\mathbf{e}_{(1)}, \mathbf{e}_{(2)}\right\}=$ $\left\{\frac{\mathbf{u}_{1}}{\left\|\mathbf{u}_{1}\right\|}, \frac{\mathbf{u}_{2}}{\left\|\mathbf{u}_{2}\right\|}\right\}$ obteniendo la nueva base astuta móvil en términos de la carta canónica local de $M$ de la siguiente forma

$$
\begin{array}{r}
\mathbf{e}_{(1)}(s)= \\
\dot{\gamma}^{1}(s) \mathbf{e}_{1}(s)+\frac{\dot{\gamma}^{2}(s) \mathbf{e}_{2}(s)}{\mathbf{e}_{(2)}(s)}=\sqrt{\frac{a_{22}(\mathbf{q})}{a_{11}(\mathbf{q})}} \dot{\gamma}^{2} \mathbf{e}_{1}(s)-\sqrt{\frac{a_{11}(\mathbf{q})}{a_{22}(\mathbf{q})}} \dot{\gamma}^{1} \mathbf{e}_{2}(s)
\end{array}
$$

Las ecuaciones (4.42) pueden ser fácilmente expresadas en esta base siendo transportada paralelamente a lo largo de la trayectoria $\gamma(s)$ resolviendo la ecuación dinámica

$$
\frac{\nabla \mathbf{e}_{(i)}}{d s} \equiv \frac{D \mathbf{e}_{(i)}}{d s}=0, \quad i=1,2
$$

con $\mathbf{e}_{(i)}=\mathbf{e}_{(i)}^{j} \mathbf{e}_{j}$ resolvemos 4.46 usando la serie de Taylor

$$
\left.\mathbf{e}_{(i)}^{\alpha}\right|_{t}=\left.\mathbf{e}_{(i)}^{\alpha}\right|_{t_{0}}-\left.\mathbf{e}_{(i)}^{\mu} \Gamma_{\mu \beta}^{\alpha}\left(\frac{d q^{\beta}}{d t}\right)\right|_{t_{0}}\left(t-t_{0}\right)+o\left(t-t_{0}\right)^{2}
$$


Si el campo vectorial $\mathbf{J}(s)$ es expresado como $\mathbf{J}(s)=J^{i}(s) \mathbf{e}_{(i)}(s)$, podemos escribir 4.42 como

$$
\begin{aligned}
& \frac{d^{2} J^{1}}{d s^{2}}=0 \\
& \frac{d^{2} J^{2}}{d s^{2}}=-\frac{R_{1212}}{\left|g_{i j}\right|} J^{2}=-\frac{\hat{R}(s)}{2} J^{2},
\end{aligned}
$$

con $\hat{R}$ la curvatura escalar para la métrica $g_{i j}$.

Fijada una longitud constante $s$ para la trayectoria, podemos definir un indicador geométrico que hemos denominado SGI (Stability Geometric Indicator) como

$$
S G I\left(s, \mathbf{q}_{\mathbf{0}}\right)=\max _{0 \leq s^{\prime} \leq s}\left(J\left(s^{\prime}, \mathbf{q}_{\mathbf{0}}\right)\right),
$$

con condiciones iniciales $\mathbf{q}_{0}$.

Utilizaremos este indicador para comparar estabilidades relativas para diferentes trayectorias.

\subsubsection{Parametrización por tiempo físico $t$}

Igualmente la ecuación 4.48 podemos referirla en lugar de a su parámetro natural al parámetro tiempo físico $(t)$ de la siguiente manera

$$
\frac{d^{2} \xi}{d t^{2}}-\frac{1}{W} \frac{d W}{d t} \frac{d \xi}{d t}+2 W^{2} \mathbf{Q} \xi=0 .
$$

Podemos sustituir

$$
\mathbf{Y} \equiv \frac{\xi(t)}{\sqrt{W}}
$$

obteniendo

$$
\begin{gathered}
\frac{d^{2} \mathbf{Y}}{d t^{2}}+\mathbf{\Omega}(t) \mathbf{Y}=0, \\
\boldsymbol{\Omega}(t)=\frac{1}{2}\left[\frac{d^{2} W}{d t^{2}}-\frac{3}{2 W}\left(\frac{d W}{d t}\right)^{2}\right] \mathbf{I}+2 W^{2} \mathbf{Q}, \\
\operatorname{con} Q_{i j}=<R\left(\mathbf{v}, \mathbf{e}_{(i)}\right) \mathbf{v}, \mathbf{e}_{(j)},>=R_{k l m n} v^{k} e_{(i)}^{l} v^{m} e_{(j)}^{n}, \quad k, l, m, n=1,2, \ldots, N
\end{gathered}
$$




\subsubsection{Dimensión tres}

Extendemos el cálculo anterior a dimensión tres

$$
\begin{aligned}
& <R\left(\mathbf{e}_{(1)}, \mathbf{e}_{(m)}\right) \mathbf{e}_{(1)}, \mathbf{e}_{(n)}>=R_{l j h k} \mathbf{e}_{(1)}^{l} \mathbf{e}_{(m)}^{j} \mathbf{e}_{(1)}^{h} \mathbf{e}_{(n)}^{k}=
\end{aligned}
$$

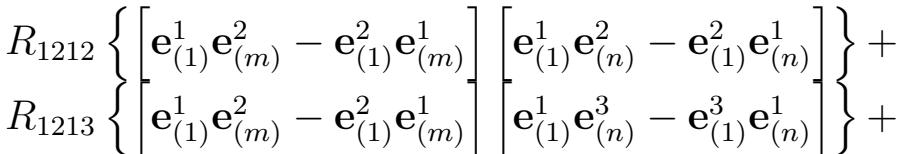

$$
\begin{aligned}
& R_{1213}\left\{\left[\mathbf{e}_{(1)}^{1} \mathbf{e}_{(n)}^{2}-\mathbf{e}_{(1)}^{2} \mathbf{e}_{(n)}^{1}\right]\left[\mathbf{e}_{(1)}^{1} \mathbf{e}_{(m)}^{3}-\mathbf{e}_{(1)}^{3} \mathbf{e}_{(m)}^{1}\right]\right\}+ \\
& R_{1223}\left\{\left[\mathbf{e}_{(1)}^{1} \mathbf{e}_{(m)}^{2}-\mathbf{e}_{(1)}^{2} \mathbf{e}_{(m)}^{1}\right]\left[\mathbf{e}_{(1)}^{2} \mathbf{e}_{(n)}^{3}-\mathbf{e}_{(1)}^{3} \mathbf{e}_{(n)}^{2}\right]\right\}+ \\
& R_{1223}\left\{\left[\mathbf{e}_{(1)}^{1} \mathbf{e}_{(n)}^{2}-\mathbf{e}_{(1)}^{2} \mathbf{e}_{(n)}^{1}\right]\left[\mathbf{e}_{(1)}^{2} \mathbf{e}_{(m)}^{3}-\mathbf{e}_{(1)}^{3} \mathbf{e}_{(m)}^{2}\right]\right\}+ \\
& R_{1313}\left\{\left[\mathbf{e}_{(1)}^{1} \mathbf{e}_{(m)}^{2}-\mathbf{e}_{(1)}^{2} \mathbf{e}_{(m)}^{1}\right]\left[\mathbf{e}_{(1)}^{2} \mathbf{e}_{(n)}^{3}-\mathbf{e}_{(1)}^{3} \mathbf{e}_{(n)}^{2}\right]\right\}+ \\
& R_{1323}\left\{\left[\mathbf{e}_{(1)}^{1} \mathbf{e}_{(m)}^{3}-\mathbf{e}_{(1)}^{3} \mathbf{e}_{(m)}^{1}\right]\left[\mathbf{e}_{(1)}^{1} \mathbf{e}_{(n)}^{3}-\mathbf{e}_{(1)}^{3} \mathbf{e}_{(n)}^{2}\right]\right\}+ \\
& R_{1323}\left\{\left[\mathbf{e}_{(1)}^{1} \mathbf{e}_{(n)}^{3}-\mathbf{e}_{(1)}^{3} \mathbf{e}_{(n)}^{1}\right]\left[\mathbf{e}_{(1)}^{2} \mathbf{e}_{(m)}^{3}-\mathbf{e}_{(1)}^{3} \mathbf{e}_{(m)}^{2}\right]\right\}+ \\
& R_{2323}\left\{\left[\mathbf{e}_{(1)}^{2} \mathbf{e}_{(m)}^{3}-\mathbf{e}_{(1)}^{3} \mathbf{e}_{(m)}^{2}\right]\left[\mathbf{e}_{(1)}^{2} \mathbf{e}_{(n)}^{3}-\mathbf{e}_{(1)}^{3} \mathbf{e}_{(n)}^{2}\right]\right\}
\end{aligned}
$$

$$
\begin{aligned}
& \left(\begin{array}{c}
\frac{d^{2} J^{1}}{d s^{2}} \\
\frac{d^{2} J^{2}}{d s^{2}} \\
\frac{d^{2} J^{3}}{d s^{2}}
\end{array}\right)+ \\
& \left(\begin{array}{ccc}
<R\left(\mathbf{v}, \mathbf{e}_{(1)}\right) \mathbf{v}, \mathbf{e}_{(1)}> & <R\left(\mathbf{v}, \mathbf{e}_{(2)}\right) \mathbf{v}, \mathbf{e}_{(1)}> & <R\left(\mathbf{v}, \mathbf{e}_{(3)}\right) \mathbf{v}, \mathbf{e}_{(1)}> \\
<R\left(\mathbf{v}, \mathbf{e}_{(1)}\right) \mathbf{v}, \mathbf{e}_{(2)}> & <R\left(\mathbf{v}, \mathbf{e}_{(2)}\right) \mathbf{v}, \mathbf{e}_{(2)}> & <R\left(\mathbf{v}, \mathbf{e}_{(3)}\right) \mathbf{v}, \mathbf{e}_{(2)}> \\
<R\left(\mathbf{v}, \mathbf{e}_{(1)}\right) \mathbf{v}, \mathbf{e}_{(3)}> & <R\left(\mathbf{v}, \mathbf{e}_{(2)}\right) \mathbf{v}, \mathbf{e}_{(3)}> & <R\left(\mathbf{v}, \mathbf{e}_{(3)}\right) \mathbf{v}, \mathbf{e}_{(3)}>
\end{array}\right)\left(\begin{array}{c}
J^{1} \\
J^{2} \\
J^{3}
\end{array}\right)=0
\end{aligned}
$$

tomando $\mathbf{v}=\mathbf{e}_{(1)}$

$$
\left(\begin{array}{c}
\frac{d^{2} J^{1}}{d s^{2}} \\
\frac{d^{d} J^{2}}{d s^{2}} \\
\frac{d^{d} J^{3}}{d s^{2}}
\end{array}\right)+\left(\begin{array}{ccc}
0 & 0 & 0 \\
0 & <R\left(\mathbf{e}_{(1)}, \mathbf{e}_{(2)}\right) \mathbf{e}_{(1)}, \mathbf{e}_{(2)}> & <R\left(\mathbf{e}_{(1)}, \mathbf{e}_{(3)}\right) \mathbf{e}_{(1)}, \mathbf{e}_{(2)}> \\
0 & <R\left(\mathbf{e}_{(1)}, \mathbf{e}_{(2)}\right) \mathbf{e}_{(1)}, \mathbf{e}_{(3)}> & <R\left(\mathbf{e}_{(1)}, \mathbf{e}_{(3)}\right) \mathbf{e}_{(1)}, \mathbf{e}_{(3)}>
\end{array}\right)\left(\begin{array}{c}
J^{1} \\
J^{2} \\
J^{3}
\end{array}\right)=0
$$




\subsection{Puntos conjugados.}

En esta sección estudiaremos la evolución del módulo del vector separación geodésico, es decir, $\|\mathbf{J}\|$ utilizando igualmente la ecuación de $J L C$

$$
\begin{gathered}
\frac{D}{d s} \frac{D}{d s} \mathbf{J}+R(\mathbf{v}, \mathbf{J}) \mathbf{v}=0 \\
\|\mathbf{J}\|^{2}=<\mathbf{J}, \mathbf{J}>=g_{i j} J^{i} J^{j} \\
\frac{d}{d s}\|\mathbf{J}\|^{2}=\frac{d}{d s}<\mathbf{J}, \mathbf{J}>=2<\frac{D}{d s} \mathbf{J}, \mathbf{J}>\Rightarrow \\
\frac{d d^{2}}{d s^{2}}\|\mathbf{J}\|^{2}=\frac{d}{d s}\left(2<\frac{D}{d s} \mathbf{J}, \mathbf{J}>\right)=2<\underbrace{\frac{D}{d s} \frac{D}{d s} \mathbf{J}, \mathbf{J}>+2<\frac{D}{d s} \mathbf{J}, \mathbf{J}>\Rightarrow}_{-R(\mathbf{v}, \mathbf{J}) \mathbf{v}} \\
\frac{d^{2}}{d s^{2}}\|\mathbf{J}\|^{2}=2\left(<-R(\mathbf{v}, \mathbf{J}) \mathbf{v}, \mathbf{J}>+\left\|\frac{D}{d s} \mathbf{J}\right\|^{2}\right)
\end{gathered}
$$

tomando como base móvil $\left\{\mathbf{e}_{T}, \mathbf{e}_{J}\right\}$, es decir, $\frac{D}{d s} \mathbf{e}_{T}=0, \frac{D}{d s} \mathbf{e}_{J}=0$,

y $\mathbf{J}=\|\mathbf{J}\| \mathbf{e}_{J}$ con $\left\|\mathbf{e}_{J}\right\|=1$, entonces

$$
\begin{gathered}
\frac{d^{2}}{d s^{2}}\|\mathbf{J}\|=\left(<-R\left(\mathbf{v}, \mathbf{e}_{J}\right) \mathbf{v}, \mathbf{e}_{J}>+\left\|\frac{D}{d s} \mathbf{e}_{J}\right\|^{2}\right) \\
\frac{d^{2}}{d s^{2}}\|\mathbf{J}\|=-K\left(\mathbf{v}, \mathbf{e}_{J}\right)\|\mathbf{J}\|
\end{gathered}
$$

$K\left(\mathbf{v}, \mathbf{e}_{J}\right)=<R\left(\mathbf{v}, \mathbf{e}_{J}\right) \mathbf{v}, \mathbf{e}_{J}>\equiv$ curvatura seccional

$$
\frac{d^{2}}{d s^{2}}\|\mathbf{J}\|=-\underbrace{K\left(\mathbf{v}, \mathbf{e}_{J}\right)}_{K(s)}\|\mathbf{J}\|
$$




$$
K\left(\mathbf{v}, \mathbf{e}_{J}\right)=<R\left(\mathbf{v}, \mathbf{e}_{J}\right) \mathbf{v}, \mathbf{e}_{J}>=<R\left(\mathbf{v}, \frac{\mathbf{J}}{\|\mathbf{J}\|}\right) \mathbf{v}, \frac{\mathbf{J}}{\|\mathbf{J}\|}>
$$

su expresión en coordenadas será

$$
\begin{gathered}
K\left(\mathbf{v}, \mathbf{e}_{J}\right)=<R\left(\mathbf{v}, \frac{\mathbf{J}}{\|\mathbf{J}\|}\right) \mathbf{v}, \frac{\mathbf{J}}{\|\mathbf{J}\|}>=R_{i j k l} \frac{J^{i}}{\|\mathbf{J}\|} \frac{d q^{j}}{d s} \frac{J^{k}}{\|\mathbf{J}\|} \frac{d q^{l}}{d s} \\
\frac{d^{2}\|\mathbf{J}\|}{d s^{2}}=-\underbrace{R_{i j k l} \frac{J^{i}}{\|\mathbf{J}\|} \frac{d q^{j}}{d s} \frac{J^{k}}{\|\mathbf{J}\|} \frac{d q^{l}}{d s}}_{K(s)}\|\mathbf{J}\|,
\end{gathered}
$$

obtenemos la Ecuación de Hill generalizada

$$
\frac{d^{2}\|\mathbf{J}\|}{d s^{2}}+K(s)\|\mathbf{J}\|=0
$$

\subsubsection{Puntos conjugados}

\section{Definición y ejemplos}

Sea la curva $\gamma:(0, a) \longmapsto M$ una geodésica.

El punto $q=\gamma\left(t_{0}\right)$ con $t_{0} \in(0, a]$ es conjugado con $p$ a lo largo de $\gamma$ si existe un campo de Jacobi $\mathbf{J}$ a lo largo de $\gamma$, no idénticamente cero, con $\mathbf{J}(0)=0=\mathbf{J}\left(t_{0}\right)$.

El máximo número de estos campos linealmente independientes se llama multiplicidad del punto conjugado $\gamma\left(t_{0}\right)$.

Ejemplo:

En la esfera $\mathbb{S}^{n}=\left\{x \in \mathbb{R}^{n+1} /|x|=1\right\}$ el campo de Jacobi en $\mathbb{S}^{n}$ será

$$
J(t)=(\sin t)(w(t))
$$

cumple $J(0)=J(\pi)=0$ por tanto el punto antipodal $\gamma(\pi)$ de $\gamma(0)$ es conjugado a $\gamma(0)$.

El conjunto de los primeros puntos conjugados al punto $p \in M$ para todas 
las geodésicas que comienzan en $p$ se denomina lugar geométrico (locus) de $p$ y se denota por $C(p)$.

\subsubsection{Ejemplos}

En la esfera $\mathbb{S}^{n} C(p)=-p, \quad \forall p$.

En el elipsoide $C(p)$ será una curva con cuatro puntos singulares

Como vemos en la ecuación de Hill generalizada 4.67) los puntos conjugados suponen posibles puntos de singularidad para la estabilidad de la trayectoria ya que $|J|=0$. El número de puntos conjugados a lo largo de una geodésica/trayectoria regular es proporcional a la longitud de la curva Cerruti-Sola et Pettini, 1996. De donde se puede deducir un indicador de caos basado en este criterio de puntos conjugados.

En los puntos conjugados no se cumple el principio de acción estacionaria, en particular para geodésicas, el hecho de traspasar un punto conjugado supone que la distancia más corta entre dos puntos ya no está garantizada por la geodésica, implicando irreversibilidad y por tanto una posible transición al caos.

\section{Espacios de curvatura constante}

Supongamos que tenemos una variedad Riemanniana de curvatura seccional constante $K$. Entonces $\frac{R_{1212}}{\left|g_{i j}\right|}=\frac{R}{2}=K$ y el sistema que debemos resolver será

$$
\begin{aligned}
& \frac{d^{2} \xi^{1}}{d s^{2}}=0 \\
& \frac{d^{2} \xi^{2}}{d s^{2}}+\underbrace{\frac{R_{1212}}{|g|}}_{\frac{R}{2}} \xi^{2}=\frac{d^{2} \xi^{2}}{d s^{2}}+K \xi^{2}=0 .
\end{aligned}
$$

Si $\mathbf{w}\left(\right.$ s) es un campo paralelo a lo largo de la geodésica $\gamma(s)$ con $<\gamma^{\prime}(s)$, w $(s)>=$ $0 \mathrm{y}\|\mathbf{w}(s)\|=1$ se puede comprobar que 


$$
\mathbf{J}(s)= \begin{cases}\frac{\sin (s \sqrt{K})}{\sqrt{K}} \mathbf{w}(s), & \text { si } K>0 \\ s \mathbf{w}(s), & \text { si } K=0 \\ \frac{\sinh (s \sqrt{-K})}{\sqrt{-K}} \mathbf{w}(s), & \text { si } K<0\end{cases}
$$

y las condiciones iniciales $\mathbf{J}(0)=0$ and $\mathbf{J}^{\prime}(0)=\mathbf{w}(0)$

\section{Problema de Kepler 2D}

Para el potencial gravitacional, es decir, $V(r)=-G \frac{M}{r}$ tenemos

$$
\begin{aligned}
& \Gamma_{11}^{1}=\frac{-G M}{2\left(G M r+E r^{2}\right)}, \\
& \Gamma_{22}^{1}=\frac{-r(G M+2 E r)}{2(G M+E r)}, \\
& \Gamma_{12}^{2}=\frac{(G M+2 E r)}{2\left(G M r+E r^{2}\right)}=\Gamma_{21}^{2} .
\end{aligned}
$$

Las ecuaciones para las geodésicas tienen la forma

$$
\begin{aligned}
\frac{d^{2} r}{d s^{2}}+\frac{-G M}{2\left(G M r+E r^{2}\right)}\left(\frac{d r}{d s}\right)^{2}+\frac{-r(G M+2 E r)}{2(G M+E r)}\left(\frac{d \theta}{d s}\right)^{2} & =0, \\
\frac{d^{2} \theta}{d s^{2}}+2 \frac{(G M+2 E r)}{2\left(G M r+E r^{2}\right)} \frac{d r}{d s} \frac{d \theta}{d s} & =0 .
\end{aligned}
$$

Transformando el parámetro longitud de arco (s) en parámetro tiempo físico (t) obtenemos la ecuaciones de Newton para el potencial radial, esto es,

$$
\begin{array}{lcc}
\frac{d^{2} r}{d t^{2}}-r\left(\frac{d \theta}{d t}\right)^{2} & = & -\frac{G M}{r^{2}} \\
r \frac{d^{2} \theta}{d t^{2}}+2 \frac{d r}{d t} \frac{d \theta}{d t} & = & 0
\end{array}
$$

la solución para este sistema será la siguiente ecuación para la cónica

$$
\begin{gathered}
r=\frac{\frac{l^{2}}{G M}}{1+e \cos \theta}, \\
r^{2} \frac{d \theta}{d t}=l,
\end{gathered}
$$

donde $e=$ excentricidad con $0<e<1$ y $l=$ momento angular (constante) 
Para que $r(s)=R$ (órbita circular de radio $R$ ) sea geodésica es necesario

$$
\begin{aligned}
\Gamma_{22}^{1}\left(\frac{d \theta}{d s}\right)^{2}=\frac{-r(G M+2 E r)}{2(G M+E r)}\left(\frac{d \theta}{d s}\right)^{2} & =0 \\
\frac{d^{2} \theta}{d s^{2}} & =0 .
\end{aligned}
$$

la primera expresión se cumplirá si $R=-\frac{G M}{2 E}$ o $E=-\frac{G M}{2 R}=\frac{1}{2} V(R)$.

Podemos calcular la curvatura escalar

$$
\frac{R_{1212}}{\left|g_{i j}\right|}=\frac{R}{2}=K_{G}=-\frac{E G M}{4(r E+G M)^{3}}
$$

con $R=$ curvatura escalar y $K_{G}=$ curvatura gaussiana

$$
\left\{\begin{array}{l}
\frac{d^{2} \xi^{1}}{d s^{2}}=0, \\
\frac{d^{2} \xi^{2}}{d s^{2}}+\underbrace{\frac{R_{1212}}{|g|}}_{\frac{R}{2}} \xi^{\theta}=\frac{d^{2} \xi^{2}}{d s^{2}}-\frac{E G M}{4(r E+G M)^{3}} \xi^{2}=0 .
\end{array}\right.
$$

Estudiemos la estabilidad de la geodésica $r(s)=R$ (órbita circular de radio $R$ ).

Para esta órbita $E=\frac{1}{2} V(R)=-\frac{G M}{2 R}$ tal que $\frac{R}{2}=K_{G}=\frac{1}{G M R} \mathrm{y}$

$$
\begin{aligned}
& \frac{d^{2} \xi^{1}}{d s^{2}}=0, \\
& \frac{d^{2} \xi^{2}}{d s^{2}}+\frac{1}{G M R} \quad \xi^{2}=0 .
\end{aligned}
$$

Entonces $\frac{1}{G M R}$ representa la frecuencia en la métrica Jacobi, cambiando el parámetro de la curva de longitud de arco $s$ a tiempo físico newtoniano $t$ recuperamos la frecuencia orbital kepleriana $\omega_{K}$

$$
\begin{aligned}
& d s=2(E-V(R)) d t=\frac{G M}{R} d t \\
& \left\{\begin{array}{l}
\frac{d^{2} \xi^{1}}{d t^{2}}=0 \\
\frac{d^{2} \xi^{2}}{d t^{2}}+\frac{G M}{R^{3}} \xi^{2}=0,
\end{array}\right.
\end{aligned}
$$




$$
\begin{aligned}
& \xi^{2}(t)=k e^{m t} \operatorname{con} m=\sqrt{\frac{G M}{R^{3}}} i \text { y } k \in \mathbb{R} \\
& R e\left(\xi^{2}(t)\right)=c_{1}\left(\frac{e^{m t}+\overline{e^{m t}}}{2}\right)=c_{1} \cos \left(\sqrt{\frac{G M}{R^{3}}} t\right)
\end{aligned}
$$

$\operatorname{Im}\left(\xi^{2}(t)\right)=c_{2}\left(\frac{e^{m t}-\overline{e^{m t}}}{2 i}\right)=c_{2} \sin \left(\sqrt{\frac{G M}{R^{3}}} t\right)(4.81)$

por lo que la solución general será

$$
\xi^{2}(t)=c_{1} \cos \left(\sqrt{\frac{G M}{R^{3}}} t\right)+c_{2} \sin \left(\sqrt{\frac{G M}{R^{3}}} t\right) .
$$

Las condiciones iniciales establecen $c_{1}=0$ and $c_{2}=\sqrt{G M R}$, por lo que la solución será

$$
\xi^{2}(t)=\sqrt{G M R} \sin \left(\sqrt{\frac{G M}{R^{3}}} t\right) .
$$

Buscamos el período $T$

$$
\begin{gathered}
\sin \left(\sqrt{\frac{G M}{R^{3}}} t\right)=\sin \left(\sqrt{\frac{G M}{R^{3}}} t+2 \Pi\right)=\sin \left(\sqrt{\frac{G M}{R^{3}}}(t+\underbrace{\frac{2 \Pi \sqrt{R^{3}}}{\sqrt{G M}}}_{T})\right) \\
T=\frac{2 \pi \sqrt{R^{3}}}{\sqrt{G M}} \Rightarrow T^{2}=\frac{4 \pi^{2}}{G M} R^{3}
\end{gathered}
$$

(La tercera ley de Kepler)

Así la frecuencia orbital y la frecuencia de desviación son las mismas, de ahí que afirmemos que $r(s)=R$ sea una órbita estable.

Si entendemos $R$ como la distancia media, entonces podemos observar que el período de la perturbación es el mismo para trayectorias planetarias cuya 
distancia media sea la misma, la curva descrita por la perturbación será una tautócrona.

A partir del problema de calcular la curva de descenso más rápido entre dos puntos dados A y B que realiza un objeto material movido exclusivamente por la fuerza de gravedad y sin rozamiento se obtiene la curva cicloide

$$
\begin{aligned}
& x=R(t-\sin t) \\
& y=R(1-\cos t)
\end{aligned}
$$

La velocidad a lo largo de la curva es

$$
\frac{d s}{d t}=2 R \sin \frac{t}{2} .
$$

El tiempo que tarda la bola en llegar al punto medio $\mathrm{C}$ se puede calcular

$$
T=\pi \sqrt{\frac{R}{g}},
$$

pero si sustituimos el valor de $g$ por

$$
g=G \frac{M}{R^{2}} \rightarrow T^{2}=\frac{4 \pi^{2}}{G M} R^{3}
$$

que es el mismo resultado que para el problema de Kepler anterior.

\section{Dimension tres}

Estudiamos la ecuación de $J L C$ tridimensional

$$
\begin{aligned}
& \left(\begin{array}{c}
\frac{d^{2} \xi^{1}}{d s^{2}} \\
\frac{d^{2} \xi^{2}}{d s^{2}} \\
\frac{d^{2} \xi^{3}}{d s^{2}}
\end{array}\right)+
\end{aligned}
$$

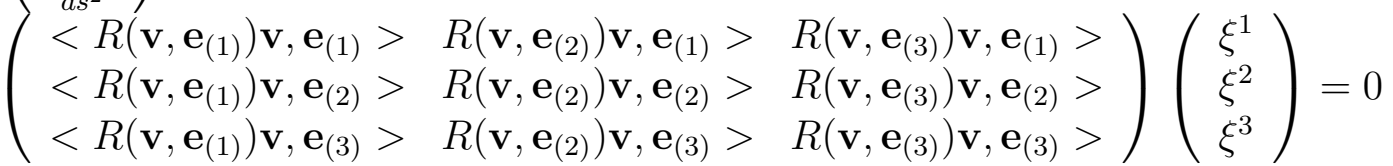

with $\mathbf{v}=\mathbf{e}_{(1)}$ then $<R\left(\mathbf{v}, \mathbf{e}_{(j)}\right) \mathbf{v}, \mathbf{e}_{(i)}>=<R\left(\mathbf{e}_{(1)}, \mathbf{e}_{(j)}\right) \mathbf{e}_{(1)}, \mathbf{e}_{(i)}>=R_{l m n p} e_{(1)}^{l} e_{(j)}^{m} e_{(1)}^{n} e_{(i)}^{p}$

$$
\left(\begin{array}{c}
\frac{d^{2} \xi^{1}}{d s^{2}} \\
\frac{d^{2} \xi^{2}}{d s^{2}} \\
\frac{d^{2} \xi^{3}}{d s^{2}}
\end{array}\right)+\left(\begin{array}{ccc}
0 & 0 & 0 \\
0 & K\left(\mathbf{e}_{(1)}, \mathbf{e}_{(2)}\right) & <R\left(\mathbf{e}_{(1)}, \mathbf{e}_{(3)}\right) \mathbf{e}_{(1)}, \mathbf{e}_{(2)}> \\
0 & <R\left(\mathbf{e}_{(1)}, \mathbf{e}_{(3)}\right) \mathbf{e}_{(1)}, \mathbf{e}_{(2)}> & K\left(\mathbf{e}_{(1)}, \mathbf{e}_{(3)}\right)
\end{array}\right)\left(\begin{array}{l}
\xi^{1} \\
\xi^{2} \\
\xi^{3}
\end{array}\right)=0
$$




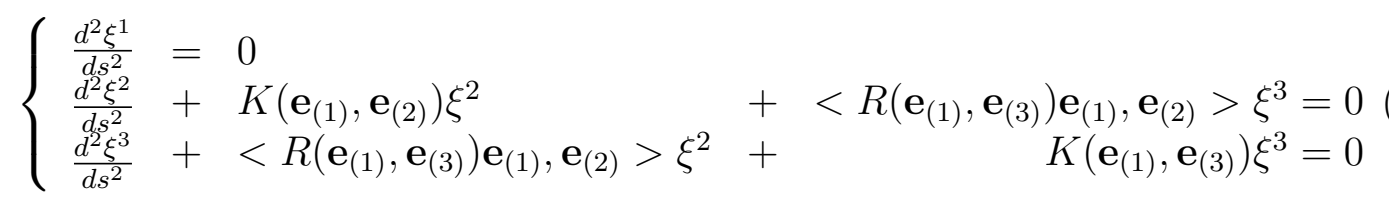

\section{Problema de Kepler 3D}

Consideremos las coordenadas esféricas $\{r, \phi, \theta\}$, que se relacionan con las coordenadas cartesianas como

$$
\left\{\begin{array}{l}
x=r \sin \phi \cos \theta \\
y=r \sin \phi \sin \theta \\
z=r \cos \phi
\end{array}\right.
$$

La base canónica para el espacio tangente será $\left\{\mathbf{e}_{r}, \mathbf{e}_{\phi}, \mathbf{e}_{\theta}\right\}$ y el diferencial de longitud es

$$
d s^{2}=d r^{2}+r^{2} d \phi^{2}+r^{2} \sin ^{2} \phi d \theta^{2}
$$

La métrica Jacobi para la (variedad mecánica) tiene la forma

$$
g_{i j}=2(E-V(r)) a_{i j}(r, \theta)=2(E-V(r))\left(\begin{array}{ccc}
1 & 0 & 0 \\
0 & r^{2} & 0 \\
0 & 0 & r^{2} \sin ^{2} \phi
\end{array}\right) .
$$

La ecuación de Jacobi-Levi-Civita (en la nueva base astuta $\left\{\mathbf{e}_{(1)}, \mathbf{e}_{(2)}, \mathbf{e}_{(3)}\right\}$ ) será

$$
\begin{array}{rlrl}
\frac{d^{2} \xi^{1}}{d s^{2}}= & 0 & & \\
\frac{d^{2} \xi^{2}}{d s^{2}}+K\left(\mathbf{e}_{(1)}, \mathbf{e}_{(2)}\right) \xi^{2} & + & \\
\frac{d^{2} \xi^{3}}{d s^{2}}+ & +<R\left(\mathbf{e}_{(1)}, \mathbf{e}_{(3)}\right) \mathbf{e}_{(1)}, \mathbf{e}_{(2)}>\xi^{2} & + & K\left(\mathbf{e}_{(1)}, \mathbf{e}_{(3)}\right) \mathbf{e}_{(1)}, \mathbf{e}_{(2)}>\xi^{3}=0, \\
& &
\end{array}
$$

Recordamos la expresión para la curvatura seccional en el punto $P$ para el subespacio dos dimensional $\sigma \subset T_{p} M$ donde $\sigma$ puede ser generado, por ejemplo, por $\mathbf{e}_{(m)}$ y $\mathbf{e}_{(n)}$ (es decir, una base de este subespacio)

$$
K(\sigma)=K\left(\mathbf{e}_{(m)}, \mathbf{e}_{(n)}\right)=\frac{R_{l j h k} e_{(m)}^{l} e_{(m)}^{h} e_{(n)}^{j} e_{(n)}^{k}}{\left(g_{l h} g_{j k}-g_{l k} g_{j h}\right) e_{(m)}^{l} e_{(m)}^{h} e_{(n)}^{j} e_{(n)}^{k}}=\frac{R_{l j h k} e_{(m)}^{l} e_{(m)}^{h} e_{(n)}^{j} e_{(n)}^{k}}{\left\|\mathbf{e}_{(m)} \times \mathbf{e}_{(n)}\right\|^{2}}
$$

donde 
$g_{i j} \equiv$ componentes ij del tensor métrico,

$R_{l j h k} \equiv$ tensor de curvatura en las coordenadas $(r, \phi, \theta)$, i.e., $R_{l j h k}=<R\left(\mathbf{e}_{l}, \mathbf{e}_{j}\right) \mathbf{e}_{h}, \mathbf{e}_{k}>$.

Por ejemplo, identificando $(1,2,3) \longleftrightarrow(r, \phi, \theta)$ tenemos

$R_{r \theta \phi r}=R_{1321}=<R\left(\mathbf{e}_{1}, \mathbf{e}_{3}\right) \mathbf{e}_{2}, \mathbf{e}_{1}>=<R\left(\mathbf{e}_{r}, \mathbf{e}_{\theta}\right) \mathbf{e}_{\phi}, \mathbf{e}_{r}>$,

$e_{(m)}^{i} \equiv$ i-ésimacomponentedelvector $\mathbf{e}_{(m)}$ de la base astuta $\left\{\mathbf{e}_{(1)}, \mathbf{e}_{(2)}, \ldots \mathbf{e}_{(n)}\right\}$

En nuestro caso con coordenadas esféricas tenemos

$$
\begin{array}{ccccc}
\mathbf{e}_{(1)}= & e_{(1)}^{1} \mathbf{e}_{r}+e_{(1)}^{2} \mathbf{e}_{\phi}+e_{(1)}^{3} \mathbf{e}_{\theta} \\
\mathbf{e}_{(2)} & =e_{(2)}^{1} \mathbf{e}_{r}+e_{(2)}^{2} \mathbf{e}_{\phi}+e_{(2)}^{3} \mathbf{e}_{\theta} \\
\vdots & \vdots & \vdots & \vdots
\end{array}
$$

tal que $e_{(i)}^{j}=<\mathbf{e}_{(i)}, \mathbf{e}_{j}>$, por ejemplo, $e_{(2)}^{3}=<\mathbf{e}_{(2)}, \mathbf{e}_{3}>=<\mathbf{e}_{(2)}, \mathbf{e}_{\theta}>$

Estudiemos la órbita circular con

$$
\left\{\begin{array}{l}
r(s)=R \\
\phi(s)=k_{1} s \quad k_{1} \in \mathbb{R}(4.99) \\
\theta(s)=\theta_{0} \quad \theta_{0} \in \mathbb{R}
\end{array}\right.
$$

y $E=\frac{1}{2} V(R)=\frac{-G M}{2 R}$.

Para simplificar todas las anteriores expresiones consideraremos la siguiente base astuta

$$
\begin{aligned}
& \mathbf{e}_{(1)}=\lambda_{1} \mathbf{e}_{\phi} \\
& \mathbf{e}_{(2)}=\lambda_{2} \mathbf{e}_{r} \\
& \mathbf{e}_{(3)}=\frac{\lambda_{3}}{\sin \phi} \mathbf{e}_{\theta}
\end{aligned}
$$

$\operatorname{con} \lambda_{1}, \lambda_{2}, \lambda_{3} \in \mathbb{R}$

Sabemos que esta base astuta $\left\{\mathbf{e}_{(1)}, \mathbf{e}_{(2)}, \mathbf{e}_{(3)}\right\}$ es una base paralelamente trasportada a lo largo de la geodésica, es decir,

$$
\frac{D \mathbf{e}_{(i)}}{d s}=0
$$


De hecho se puede comprobar que

$$
\begin{aligned}
& \frac{D \mathbf{e}_{(1)}}{d s}=\left(\frac{d e_{(1)}^{1}}{d s}+\Gamma_{i j}^{2} e_{(1)}^{j} \frac{d x^{i}}{d s}\right) \mathbf{e}_{2}=0, \\
& \frac{D \mathbf{e}_{(2)}}{d s}=\left(\frac{d e_{(2)}^{1}}{d s}+\Gamma_{i j}^{1} e_{(2)}^{j} \frac{d x^{i}}{d s}\right) \mathbf{e}_{1}=0, \\
& \frac{D \mathbf{e}_{(3)}}{d s}=\left(\frac{d e_{(3)}^{1}}{d s}+\Gamma_{i j}^{1} e_{(3)}^{j} \frac{d x^{i}}{d s}\right) \mathbf{e}_{3}=0 .
\end{aligned}
$$

Si queremos una base ortonormal es necesario

$$
\left\|\mathbf{e}_{(i)}\right\|=\sqrt{<\mathbf{e}_{(i)}, \mathbf{e}_{(i)}>}=1 \text { and }<\mathbf{e}_{(i)}, \mathbf{e}_{(j)}>=0 \text { if } i \neq j .
$$

Por tanto la base será

$$
\begin{aligned}
& \mathbf{e}_{(1)}=\lambda_{1} \mathbf{e}_{\phi}=\frac{1}{\sqrt{G M R}} \mathbf{e}_{\phi} \\
& \mathbf{e}_{(2)}=\lambda_{2} \mathbf{e}_{r}=\sqrt{\frac{R}{G M}} \mathbf{e}_{r} \\
& \mathbf{e}_{(3)}=\frac{\lambda_{3}}{\sin \phi} \mathbf{e}_{\theta}=\frac{1}{\sin \phi \sqrt{G M R}} \mathbf{e}_{\theta}, \\
& K\left(\mathbf{e}_{(1)}, \mathbf{e}_{(2)}\right)=\frac{R_{1212}}{g_{22} g_{11}-g_{12}^{2}}=\frac{1}{G M R}, \\
& K\left(\mathbf{e}_{(1)}, \mathbf{e}_{(3)}\right)=\frac{R_{2323}}{g_{22} g_{33}-g_{23}^{2}}=\frac{1}{G M R} \\
& <R\left(\mathbf{e}_{(1)}, \mathbf{e}_{(2)}\right) \mathbf{e}_{(1)}, \mathbf{e}_{(3)}>=0 \text {. } \\
& \left\{\begin{array}{l}
\frac{d^{2} \xi^{1}}{d s^{2}}=0 \\
\frac{d^{2} \xi^{2}}{d s^{2}}+\frac{1}{G M R} \xi^{2}=0 \\
\frac{d^{2} \xi^{3}}{d s^{2}}+\frac{1}{G M R} \xi^{3}=0
\end{array}\right.
\end{aligned}
$$

Entonces $\frac{1}{G M R}$ representa la frecuencia en la métrica Jacobi para $\xi^{2}$ y $\xi^{3}$, cambiando el parámetro de la curva a tiempo newtoniano $t$ recuperamos la 
frecuencia orbital Kepleriana $\omega_{K}$

$$
\begin{gathered}
d s=2(E-V(R)) d t=\frac{G M}{R} d t, \\
\left\{\begin{array}{l}
\frac{d^{2} \xi^{1}}{d t^{2}}=0 \\
\frac{d^{2} \xi^{2}}{d t^{2}}+\frac{G M}{R^{3}} \xi^{2}=0(4.107) \\
\frac{d^{2} \xi^{3}}{d t^{2}}+\frac{G M}{R^{3}} \xi^{3}=0
\end{array}\right. \\
\xi^{2}(t)=\xi^{3}(t)=k e^{m t} \operatorname{con} m=\sqrt{\frac{G M}{R^{3}} i \mathrm{y}} \\
\operatorname{Re}\left(\xi^{2}(t)\right)=\operatorname{Re}\left(\xi^{3}(t)\right)=\frac{k e^{m t}+\overline{k e^{m t}}}{2}=\cos (m t)=\cos \left(\sqrt{\frac{G M}{R^{3}}} t\right)
\end{gathered}
$$

buscamos el período $T$

$$
\cos \left(\sqrt{\frac{G M}{R^{3}}} t\right)=\cos \left(\sqrt{\frac{G M}{R^{3}}} t+2 \Pi\right)=\cos \left(\sqrt{\frac{G M}{R^{3}}}(t+\underbrace{\frac{2 \Pi \sqrt{R^{3}}}{\sqrt{G M}}}_{T})\right)
$$

por tanto

$$
T_{\xi^{2}}=T_{\xi^{3}}=\frac{2 \Pi \sqrt{R^{3}}}{\sqrt{G M}} \Rightarrow T_{\xi^{2}}^{2}=T_{\xi^{3}}^{2}=\frac{4 \Pi}{G M} R^{3} \Rightarrow T_{\xi^{2}}^{2}=T_{\xi^{3}}^{3} \propto R^{3} .
$$

Por tanto, la frecuencia orbital y la frecuencia de desviación son la misma, por tanto $r(s)=R$ es una órbita estable. 


\section{Capítulo 5}

\section{Modelo de isomerización LiNC-LiCN}

\subsection{Descripción del modelo}

El sistema que hemos elegido para ilustrar nuestro método es el sistema de isomerización LiNC-LiCN.

Esta molécula presenta dos isómeros estables ambos con configuración lineal, $\mathrm{Li}-\mathrm{N}-\mathrm{C}$ y $\mathrm{Li}-\mathrm{C}-\mathrm{N}$, separados por una barrera energética pequeña de solamente 0.0157376 a.u.

El átomo de litio puede explorar fácilmente amplias regiones anarmónicas de la energía potencial, incluso rotando alrededor de la estructura CN. De esta forma, el caos surge a muy baja energía de la energía de excitación. Es más, la frecuencia de vibración del CN es muy alta, por lo que este modo está prácticamente desacoplado del resto de vibraciones de la molécula. Como resultado, las vibraciones de este sistema molecular pueden ser estudiados adecuademante a través de un modelo de dos grados de libertad con la distancia CN congelada en su valor de equilibrio $\left(r_{e}=2,186\right.$ a.u.). La configuración geométrica se detalla en Fig. (5.1).

El correspondiente $(J=0)$ Hamiltonian clásico está dado por

$$
H=\frac{P_{R}^{2}}{2 \mu_{1}}+\frac{1}{2}\left(\frac{1}{\mu_{1} R^{2}}+\frac{1}{\mu_{2} r_{e}^{2}}\right) P_{\theta}^{2}+V(R, \theta),
$$

donde $R$ es la distancia desde el centro de masa de $\mathrm{CN}$ al átomo de li, $r_{e}$ es la distancia de equilibrio $\mathrm{CN}$, y $\theta$ es el ángulo definido por los vectores correspondientes. También, $P_{R}$ y $P_{\theta}$ son los momentos conjugados asociados, 


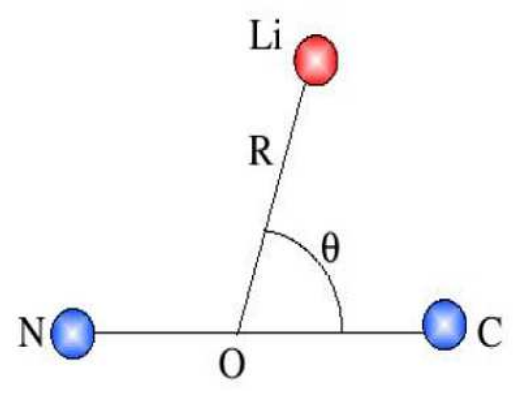

Figura 5.1: Configuración geométrica del sistema molecular LiNC-LiCN
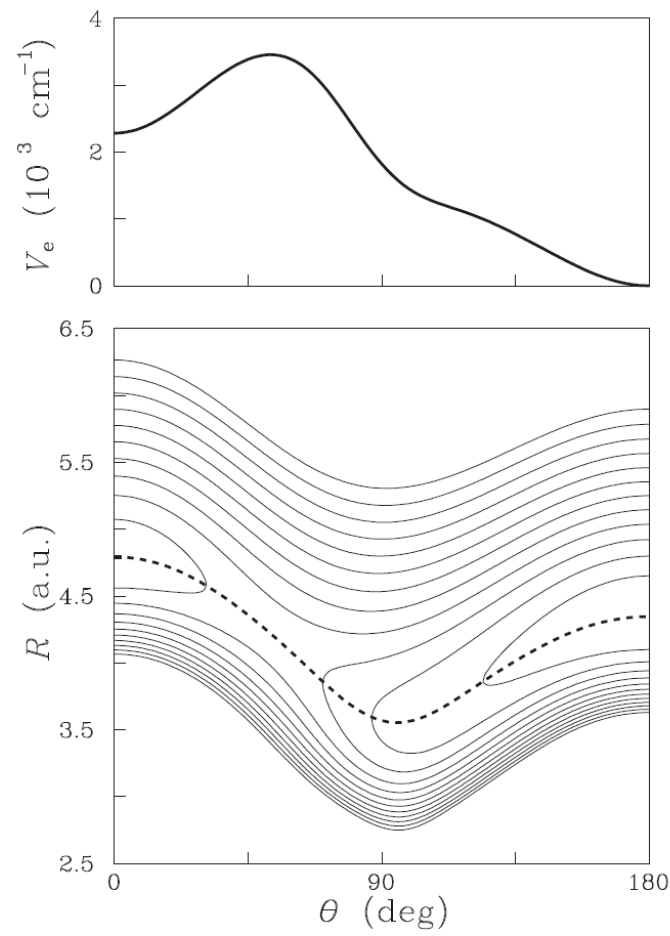

Figura 5.2: (Imagen inferior) Superficie de energía potencial para el sistema de isomerización LiNC-LiCN. Las líneas de nivel han sido representados cada $1000 \mathrm{~cm}^{-1}$. El camino de mínima energía conectando los dos isómeros se muestra como línea discontinua, (Imagen superior) Perfil de energía a lo largo del camino de mínima energía.

y $\left(\mu_{1}\right)^{-1}=m_{\mathrm{Li}}^{-1}+m_{\mathrm{CN}}^{-1} \mathrm{y}\left(\mu_{2}\right)^{-1}=m_{\mathrm{C}}^{-1}+m_{\mathrm{N}}^{-1}$ representan las masas reducidas de $\mathrm{Li}-\mathrm{CN}$ y $\mathrm{C}-\mathrm{N}$, respectivamente. La superficie de energía potencial, $V(R, \theta)$, consiste en la siguiente expresión analítica en términos de polinomios de Legendre,

$$
V(R, \theta)=\sum_{\lambda=0}^{9} v_{\lambda}(R) P_{\lambda}(\cos \theta),
$$

con coeficientes encontrados a través de cálculos mecano-cuánticos ab initio, 
Tabla 5.1: Valores numéricos de los coeficientes en la serie de Fourier para el camino de mínima energía de la Ecuación (5.3).

\begin{tabular}{rr}
\hline \hline$\lambda$ & \multicolumn{1}{c}{$b_{\lambda}$} \\
\hline 0 & 4.132893 \\
1 & 0.234366 \\
2 & 0.485224 \\
3 & -0.0163719 \\
4 & -0.0587970 \\
5 & 0.00622071 \\
6 & 0.0151456 \\
7 & -0.00178066 \\
8 & -0.00431250 \\
9 & 0.000255183 \\
\hline
\end{tabular}

y tomados de la literatura Esser et al., 1982, y se muestra en Fig. 5.2 en forma de representación de líneas de nivel. Como puede observarse, presenta dos mínimos en $\theta=0$ y $180^{\circ}$, correspondiendo a los dos isómeros lineales LiCN and LiNC, respectivamente. El camino de mínima energía, $R_{e}(\theta)$, (minimun energy path (MEP)) que conecta estos dos isómeros viene dada por la expresión

$$
R_{e}(\theta)=\sum_{\lambda=0}^{9} b_{\lambda} \cos (\lambda \theta)
$$

donde los valores numéricos para los coeficientes se indican en la Tabla 5.1 , también.

\section{Calculo de trayectorias clásicas}

Para ayudar en la discusión y con el fin de comparar utilizaremos algunos cálculos de trayectorias standard. Estos cálculos los realizaremos utilizando el algoritmo de Runge-Kutta de cuarto orden. El movimiento en la coordenada angular, $\theta$, es muy variado y por tanto el caos surge a unos niveles de energía muy bajos.

Esta dinámica se visualiza muy bien utilizando la superficie de sección de Poincaré (PSS), calculada tomando el camino de mínima energía, $R_{e}(\theta)$, como plano seccional Dai et Field, 1995]. Esta PSS define una aplicación que preserva el área definiendo un nuevo conjunto de variables canónicas.

$$
\begin{array}{ll}
\rho=R-R_{e}(\theta), & P_{\rho}=P_{R}, \\
\psi=\theta, & P_{\psi}=P_{\theta}-\left(\frac{d R_{e}}{d \theta}\right)_{\theta=\psi} P_{\rho} .
\end{array}
$$

La PSS se define ahora imponiendo la condición $\rho=0$, con $P_{\rho}$ predeterminada de antemano (en nuestros cálculos por valores negativos) de la ecuación de 
segundo orden para $P_{\rho}$ que surge de la conservación de la energía a través del hamiltoniano $H\left(\rho, \psi, P_{\rho}, P_{\psi}\right)=E$.

Finalmente, todos los puntos de la PSS son doblados en el intervalo $\psi \in$ $\left(0,180^{\circ}\right)$ para tener en cuenta la simetría del sistema molecular Dai et Field, 1995.

\subsection{Formulación geometrodinámica del mode- lo LiNC-LiCN}

Para nuestro hamiltoniano (5.1) la inversa de la matriz de masa es

$$
\left(a^{i j}\right)=\left(\begin{array}{cc}
a^{R R} & 0 \\
0 & a^{\theta \theta}
\end{array}\right)
$$

donde $a^{R R}=\mu_{1}^{-1}$ y $a^{\theta \theta}=\left(\mu_{1} R^{2}\right)^{-1}+\left(\mu_{2} r_{e}^{2}\right)^{-1}$, la variedad Riemanniana, $\left(M, g_{i j}\right)$, se define como: $M=\left\{(R, \theta) \in \mathbb{R}^{2} / V(R, \theta)<E\right\}$ y

$g_{i j}(R, \theta)=2 W a_{i j}(R, \theta)$ con $W=2(E-V(R, \theta))$, y los símbolos de Christoffel para esta métrica son

$$
\begin{aligned}
\Gamma_{R R}^{R} & =-\frac{1}{2 W} \frac{\partial V}{\partial R} \\
\Gamma_{R \theta}^{R} & =\Gamma_{\theta R}^{R}=-\frac{1}{2 W} \frac{\partial V}{\partial \theta} \\
\Gamma_{\theta \theta}^{R} & =\frac{\mu_{2} R r_{e}^{2}\left[-2 \mu_{2} r_{e}^{2} W+R\left(\mu_{1} R^{2}+\mu_{2} r_{e}^{2}\right) \frac{\partial V}{\partial R}\right]}{2\left(\mu_{1} R^{2}+\mu_{2} r_{e}^{2}\right)^{2} W} \\
\Gamma_{R R}^{\theta} & =\frac{\left(\mu_{1} R^{2}+\mu_{2} r_{e}^{2}\right) \frac{\partial V}{\partial \theta}}{2 \mu_{2} R^{2} r_{e}^{2} W} \\
\Gamma_{R \theta}^{\theta} & =\Gamma_{\theta R}^{\theta}=\frac{2 \mu_{2} r_{e}^{2} W-R\left(\mu_{1} R^{2}+\mu_{2} r_{e}^{2}\right) \frac{\partial V}{\partial R}}{2 R\left(\mu_{1} R^{2}+\mu_{2} r_{e}^{2}\right) W} \\
\Gamma_{\theta \theta}^{\theta} & =-\frac{1}{2 W} \frac{\partial V}{\partial \theta}
\end{aligned}
$$

Calculamos las trajectorias parametrizadas por tiempo físico $t$ integrando las ecuaciones

$$
\begin{array}{r}
\frac{d^{2} R}{d t^{2}}+\frac{1}{W}\left(\frac{\partial V}{\partial R} \frac{d R}{d t}+\frac{\partial V}{\partial \theta} \frac{d \theta}{d t}\right) \frac{d R}{d t}+ \\
+\Gamma_{R R}^{R}\left(\frac{d R}{d t}\right)^{2}+2 \Gamma_{R \theta}^{R} \frac{d R}{d t} \frac{d \theta}{d t}+\Gamma_{\theta \theta}^{R}\left(\frac{d \theta}{d t}\right)^{2}=0 \\
\frac{d^{2} \theta}{d t^{2}}+\frac{1}{W}\left(\frac{\partial V}{\partial R} \frac{d R}{d t}+\frac{\partial V}{\partial \theta} \frac{d \theta}{d t}\right) \frac{d \theta}{d t}+ \\
+\Gamma_{R R}^{\theta}\left(\frac{d R}{d t}\right)^{2}+2 \Gamma_{R \theta}^{\theta} \frac{d R}{d t} \frac{d \theta}{d t}+\Gamma_{\theta \theta}^{\theta}\left(\frac{d \theta}{d t}\right)^{2}=0
\end{array}
$$




\subsubsection{Las ecuaciones JLC}

Nos centraremos en el estudio del campo de separación geodésica, $\mathbf{J}(s)=$ $J^{1}(s) \mathbf{e}_{(1)}(s)+J^{2}(s) \mathbf{e}_{(2)}(s)$, a lo largo de una trayectoria de referencia $\gamma(s)$, obteniendo para el escalar $J$ la siguiente ecuación JLC en términos de tiempo físico $t$

$$
\frac{d^{2} J}{d t^{2}}-\frac{1}{W} \frac{d W}{d t} \frac{d J}{d t}+2 W^{2} \hat{R} J=0
$$

donde la curvatura escalar, $\hat{R}$, en cualquier punto de la variedad determina la inestabilidad local de las geodésicas alrededor de la trayectoria de referencia, y viene dada por

$$
\hat{R}=\frac{1}{2 W} \tilde{\mathcal{R}}+\frac{(N-1)}{8 W^{3}}\left\{4 W \nabla^{2} V+(6-N)|\nabla V|^{2}\right\},
$$

siendo $N$ la dimensión del sistema ( $N=2$ en nuestro caso), $\tilde{\mathcal{R}}=6 r_{e}^{2} \mu_{2} /\left(r_{e}^{2} \mu_{2}+\right.$ $\left.\mu_{1} R^{2}\right)^{2}$ la curvatura escalar para el espacio con métrica $a_{i j}(R, \theta),|\nabla V|^{2}=$ $a^{i j} \partial_{i} V \partial_{j} V$, and $\nabla^{2}$ el operador de Laplacian-Beltrami

$$
\nabla^{2} V=a^{i j} \nabla_{i} \nabla_{j} V=a^{i j}\left(\frac{\partial^{2} V}{\partial x^{i} \partial x^{j}}-\tilde{\Gamma}_{j i}^{k} \frac{\partial V}{\partial x^{k}}\right)
$$

con $\tilde{\Gamma}_{j i}^{k}$ los símbolos de Christoffel para la métrica $a_{i j}(R, \theta)$

$$
\tilde{\Gamma}_{\theta \theta}^{R}=\frac{-r_{e}^{4} \mu_{2}^{2} R}{\left(r_{e}^{2} \mu_{2}+\mu_{1} R^{2}\right)^{2}}, \quad \tilde{\Gamma}_{R \theta}^{\theta}=\tilde{\Gamma}_{\theta R}^{\theta}=\frac{r_{e}^{2} \mu_{2}}{\left(r_{e}^{2} \mu_{2}+\mu_{1} R^{2}\right) R}
$$

obteniéndose el Laplaciano

$$
\nabla^{2} V=a^{R R} \frac{\partial^{2} V}{\partial R^{2}}+a^{\theta \theta} \frac{\partial^{2} V}{\partial \theta^{2}}+\frac{r_{e}^{2} \mu_{2}}{\left(r_{e}^{2} \mu_{2}+\mu_{1} R^{2}\right) \mu_{1} R} \frac{\partial V}{\partial R}
$$

Sustituyendo todas estas expresiones en $(5.9)$ se obtiene

$$
\begin{aligned}
\hat{R}= & \frac{1}{2 W} \frac{6 r_{e}^{2} \mu_{2}}{\left(r_{e}^{2} \mu_{2}+\mu_{1} R^{2}\right)^{2}}+\frac{1}{2 W^{2}}\left\{a^{R R} \frac{\partial^{2} V}{\partial R^{2}}+a^{\theta \theta} \frac{\partial^{2} V}{\partial \theta^{2}}+\right. \\
+\frac{r_{e}^{2} \mu_{2}}{\left(r_{e}^{2} \mu_{2}+\mu_{1} R^{2}\right) \mu_{1} R} \frac{\partial V}{\partial R} & +\frac{1}{2 W^{3}}\left\{a^{R R}\left(\frac{\partial V}{\partial R}\right)^{2}+a^{\theta \theta}\left(\frac{\partial V}{\partial \theta}\right)^{2}\right\} .
\end{aligned}
$$

Las ecuaciones diferenciales que necesitamos integrar simultaneamente en nuestro estudio geométrico son (5.7) y (5.8). Esto lo hacemos numéricamente utilizando el método Runge-Kutta de cuarto orden escrito en Fortran Numerical Recipes, 1996. 

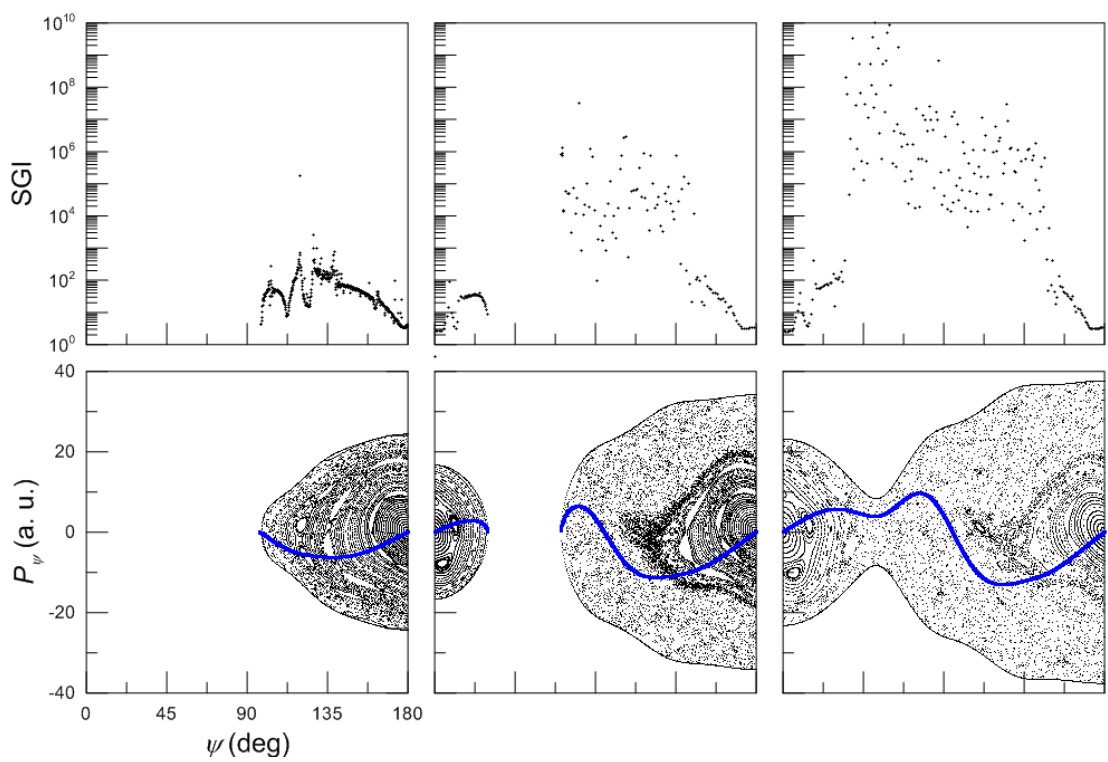

Figura 5.3: (Imagen inferior) Composición de superficies de sección de Poincaré para la dinámica vibracional para tres valores de la energía: (a) $E=1510,5$, (b) 2964.7, y (c) $3595.06 \mathrm{~cm}^{-1}$, respectivamente.

(Imagen superior) Valores numéricos del indicador SGI a las mismas energías para diferentes trayectorias comenzando en las superficies de sección inferior a lo largo de la linea $\dot{\theta}=0$ (azul).

\subsection{Aplicación del indicador SGI al modelo LiNC- LiCN}

\subsubsection{Trayectorias clásicas para LiNC-LiCN}

Consideraremos la dinámica vibración del sistema LiNC-LiCN para tres valores diferentes de energía de excitación. Los resultados se muestran en la parte inferior de la Fig. 5.3. Como se puede ver, a menor nivel de energía considerada, $E=1510,5 \mathrm{~cm}^{-1}$, (ver panel de izquierda) la mayoría de las trayectorias son regulares alrededor del $\operatorname{LiNC}\left(\theta=180^{\circ}\right)$, confinado a toros invariantes de cadenas de islas con estrechas bandas de caos. Cuando la energía aumenta a $2964.7 \mathrm{~cm}^{-1}$, (ver panel central) el movimiento en otro pozo (LiCN at $\theta=0$ ) es posible, siendo muy regular.

En lo que toca al isómero LiNC una gran zona (región exterior) del espacio de fases disponible es caótico, y se observan una notable acumulación de puntos alrededor de la región regular (interna); siendo esto una indicación de la existencia de un cantoro o barrera parcial para el flujo de trayectorias.

Finalmente, para el mayor valor de energía (panel derecho), los dos pozos están conectados por trayectorias isomerizantes, el cantoro aparece más destruido, y el caos está más distribuido, en general. 


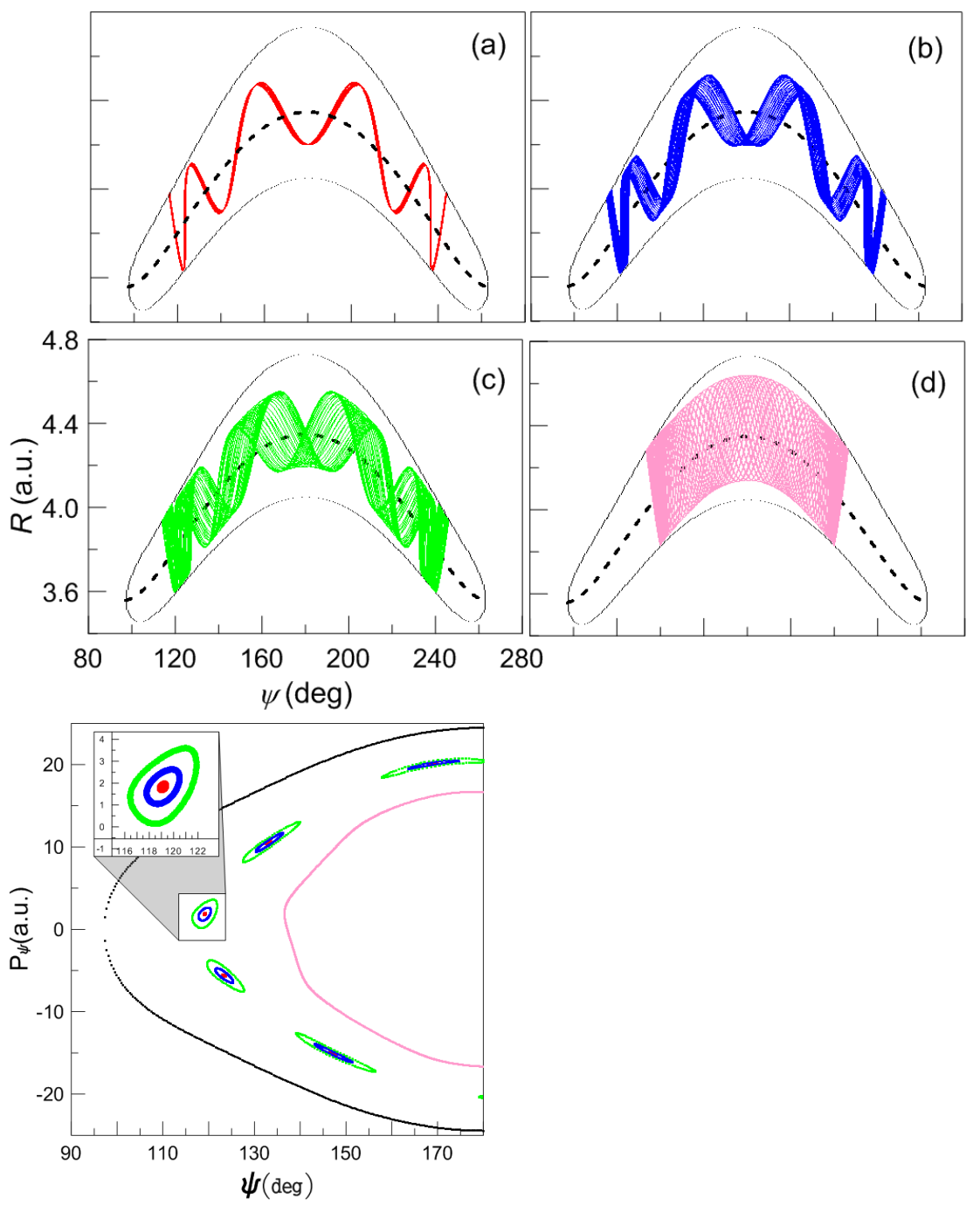

Figura 5.4: Cuatro trayectorias representativas del modelo LiNC-LiCN, y sus correspondientes superficies de sección de Poincaré para una energía $E=1510,5 \mathrm{~cm}^{-1}$.

\subsubsection{Método geométrico aplicado a trayectorias LiNC y LiCN}

En esta subsección, estudiamos algunas trayectorias representativas del sistema de isomerización LiNC-LiCN, que son analizadas utilizando el método geométrico descrito previamente. En particular, consideraremos la evolución del vector de desviación.

Empezamos con cuatro trayectorias calculadas a energía $E=1510,5 \mathrm{~cm}^{-1}$; las correspondientes órbitas [panels (a)-(d)] y la PSS [panel (e)] se muestran en Fig. 5.4. Como se ve, las primeras tres están centradas en la cadena de islas asociadas a la resonancia $\nu_{R}: \nu_{\theta}=1: 10$, estando progresivamente [de rojo a 


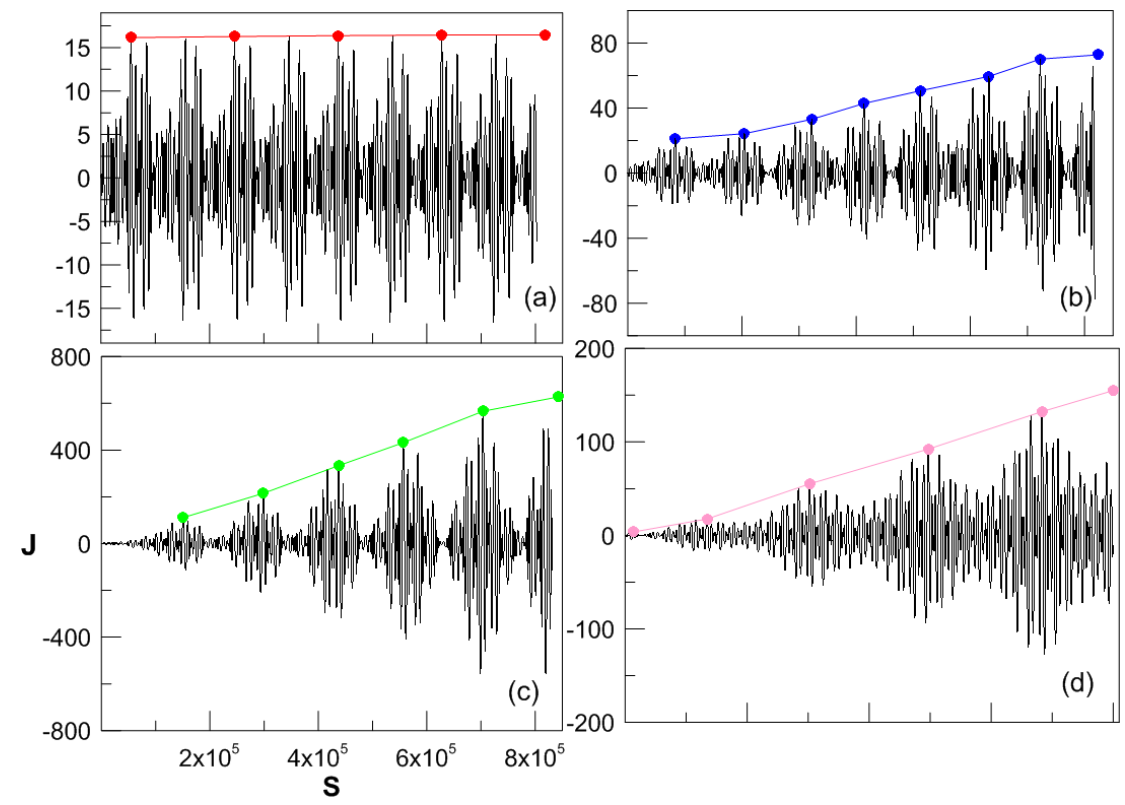

Figura 5.5: Evolución del campo vectorial de desviación $J$ con $s$ para las cuatro trayectorias LiNC-LiCN en Fig. 6.3. El comportamiento general medido con los máximos en las oscilaciones de baja frecuencia de $J$ se presenta en el panel (e).

azul a verde, y del panel (a) a (c)] más lejos desde su centro.

La última, mostrada en el panel (d), es cuasiperiódica y está confinada en el toro representado por una línea rosa en la PSS. Las evoluciones correspondientes a $J$ (la componente $J^{2}$ del vector $\mathbf{J}$ ), obteniendo el campo vectorial de desviación para las cuatro trayectorias previas, se muestran en los cuatro paneles superiores de Fig. 5.5. Como se ve esta magnitud oscila bastante con $s$, siendo la amplitud modulada por dos frecuencias principales de magnitudes muy diferentes.

Para seguir el comportamiento general, tomamos de estas representaciones previas los puntos correspondientes a los máximos en las oscilaciones de baja frecuencia. Como ayuda hemos marcado estos puntos con los mismos colores usados en Fig. 5.4 y los hemos unido a través de una poligonal.

El correspondiente polígono, mostrando el comportamiento general de $J$ con $s$, se presenentan juntos para poder comparar en la parte inferior del panel de la figura. Puede verse como la desviación de la trayectoria de referencia es aproximadamente constante para la trayectoria (a), que es prácticamente una trayectoria periódica, mientras que crece linealmente con $s$ para el resto de trayectorias resonantes y cuasiperiódicas.

Este resultado es razonable, ya que tales trayectorias están más próximas a la banda de caos asociada con la resonancia, mientras que el resto están están todas incluidas en regiones de movimiento regular.

El caso extremo es la trayectoria (a), que está casi en el centro de la isla 

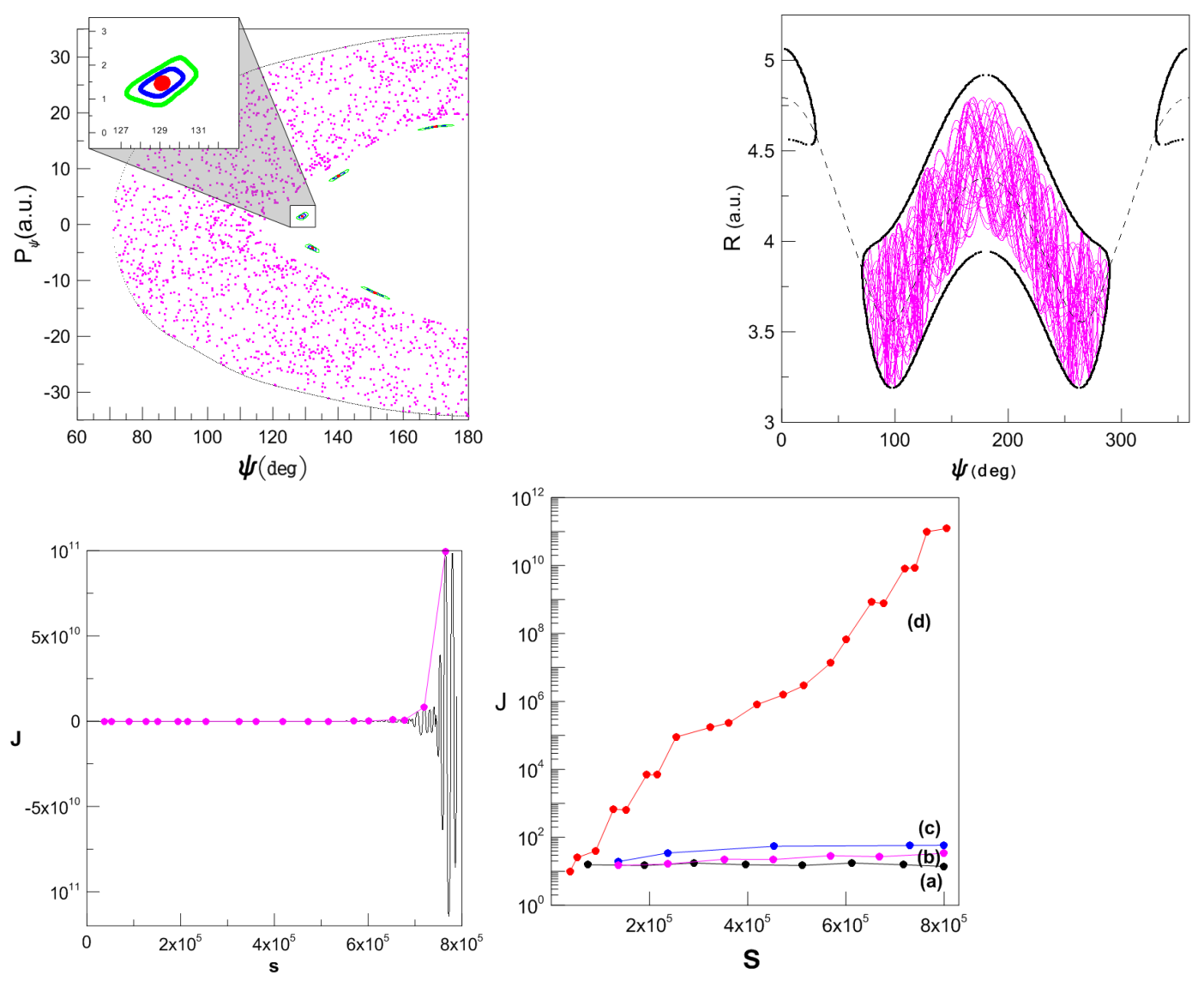

Figura 5.6: Color igual que en Figs. 5.4 y 5.5 para $E=2964,7 \mathrm{~cm}^{-1}$. Las superficies de sección de Poincaré se muestran en el panel (a), la trayectoria y $J$ vs. $s$ (a.u.) para la órbita caótica se muestran en los paneles (b) y (c), respectivamente, y el comportamiento general de $J$ para las cuatro trayectorias se puede ver en la figura (d).

resonante. Observamos también que la pendiente para la trayectoria cuasiperiódica (d) es notablemente menor que para la (c), una prueba más de la capacidad de $J$ como indicador de regularidad y caos.

Seguidamente consideramos cuatro trayectorias representativas del modelo dinámico LiNC-LiCN con $E=2964,7 \mathrm{~cm}^{-1}$. Tres de ellas son regulares, y de nuevo en la cadena de islas de la resonancia 1:10 considerada antes, mientras que la cuarta es caótica.

Las correspondientes PSS se muestran en el panel superior izquierdo de la Fig. 5.6. Además, la órbita y el vector desviación $J$ para la trayectoria caótica (rosa o figura (d)) también se muestran en los paneles superior derecho e inferior izquierdo de la figura, respectivamente. Como se puede observar, $J$ crece exponencialmente en este caso, haciendo que $J$ alcance un valor elevado para $s \gtrsim 800000$ a.u. (el valor al cual hemos parado nuestras trayectorias), a diferencia de lo que pasa en el caso regular, donde el crecimiento es solamente lineal. 
Finalmente el último panel de la figura muestra, en escala semilogarítmica, el comportamiento global de $J$ para las cuatro trayectorias consideradas.

Podemos comentar lo mismo para la energía $E=1510,5 \mathrm{~cm}^{-1}$ en relación a las trayectorias regulares. Así, el crecimiento para la trayectoria caótica es, como esperábamos, mucho mayor y exponencial (opuesto al crecimiento lineal observado para el regimen regular) que en el resto de casos.

Si examinamos más en detalle el comportamiento de $J$ para la trayectoria caótica observamos un comportamiento muy interesante, mostrando la existencia de tres regiones diferentes con diferentes valores para la pendiente. Hasta el valor del parámetro $\gtrsim 2,5 \times 10^{5}$ a.u. la trayectoria comienza con un valor definido de la pendiente. Tras este valor del parámetro la pendiente disminuye y posteriormente al valor $5,5 \times 10^{5}$ a.u. la pendiente aumenta recuperando su valor original. Este importante cambio indica la existencia de algún tipo de peculiaridad en la dinámica de esta trayetoria caótica, que parece evolucionar desde un regimen caótico a uno menos caótico de manera alternada.

Para probar esta conjetura, hemos seguido la evolución de la trayectoria en el espacio de fases en los tres periodos con el mismo valor de la pendiente descrita anteriormente. Los resultados se muestran en la Fig. 5.7 en la que se observa claramente que en diferentes periodos la trayectoria está realmente confinada en regiones diferentes del espacio de fases. Durante el primer y tercer periodos, correspondientes a $2,5 \times 10^{5}$ a.u. y $5,5 \times 10^{5}$ a.u. respectivamente la trayectoria está confinada en la parte exterior de la región caótica del espacio de fases (ver panel superior derecho de Fig. 5.6). Sin embargo en el periodo intermedio entre ellos, esto es, $2,5 \times 10^{5}<s<5,5 \times 10^{5}$ a.u. la trayectoria está confinada en una región estrecha e interior más próxima a la parte regular del espacio de fases, esto justifica porqué el valor de la pendiente de la evolución de $J$ es significativamente menor.

Obviamente la frontera entre estas dos regiones es el cantoro que actúa como una barrera dinámica parcial en el espacio de fases. Estas estructuras del espacio de fases son muy importantes en las vibraciones de pequeñas moléculas porque juegan el papel de controlar la energía de relajación itermolecular que suele darse en la reactividad química. Realmente son reponsable de los muchos efectos no estadísticos que deben tenerse en cuenta en la teoría estadística, como la de Rice-Ramsperger-Kassel-Marcus Rice and Ramsperger, 1927, Kassel, 1928, Marcus, 1953. La presencia de cantoros implica la existencia de correlaciones y relajaciones lentas de la dinámica en régimen caótico. Esto causa que algunas trayectorias se queden temporalmente atrapadas dentro o fuera de estas barreras durante muchos periodos de vibración. Este lapso temporal suele ser largo en comparación al descrito por el exponente de Lyapunov de separación de trayectorias. 


\subsubsection{El indicador $S G I$ para el sistema molecular LiNC- LiCN}

Para examinar el comportamiento del indicador SGI, calcularemos este indicador para trayectorias para diferentes valores de la energía vibracional y condiciones iniciales del sistema molecular LiNC-LiCN. Analizaremos un conjunto de trayectorias extraídas de aquellas utilizadas para construir la PSS en el panel inferior de la Fig. 5.3. En particular tomaremos órbitas en el MEP de la energía potencial LiNC-LiCN (en línea discontinua de la Fig. 5.2) $(\rho=0)$ y $\dot{\theta}=0$ (este valor tomado por simplicidad).

Esta elección es equivalente a

$$
P_{\psi}=-\sqrt{2\left[E-V\left(R_{e}(\theta), \theta\right)\right] a_{R R}} \frac{d R_{e}(\theta)}{d \theta} .
$$

Los correspondientes valores se muestran como función de $\psi$ (líneas azules) en los paneles inferiores de Fig. 5.3. Los valores del SGI se calculan después de que cada trayectoria haya recorrido una longitud total de $s=8 \times 10^{8}$ a.u. Así obtenemos una representación global de la estabilidad del sistema, que podemos comparar con los resultados de las correspondientes PSOSs. Los resultados se muestran en los paneles superiores de la Fig. 5.3.

Discutimos ahora el panel izquierdo de la Fig. 5.3 que se corresponde con el menor nivel de energía considerada $E=1510,5 \mathrm{~cm}^{-1}$, donde la dinámica es casi toda regular, como se observa en la PSOS. Este hecho se observa en los valores pequeños para el SGI. Es más, el indicador $S G I$ es continuo, observándose también una relativa variación cuave con la variable $\psi$. La excepción ocurre in los entornos de la resonancia, donde el valor en el centro es menor (mínimo

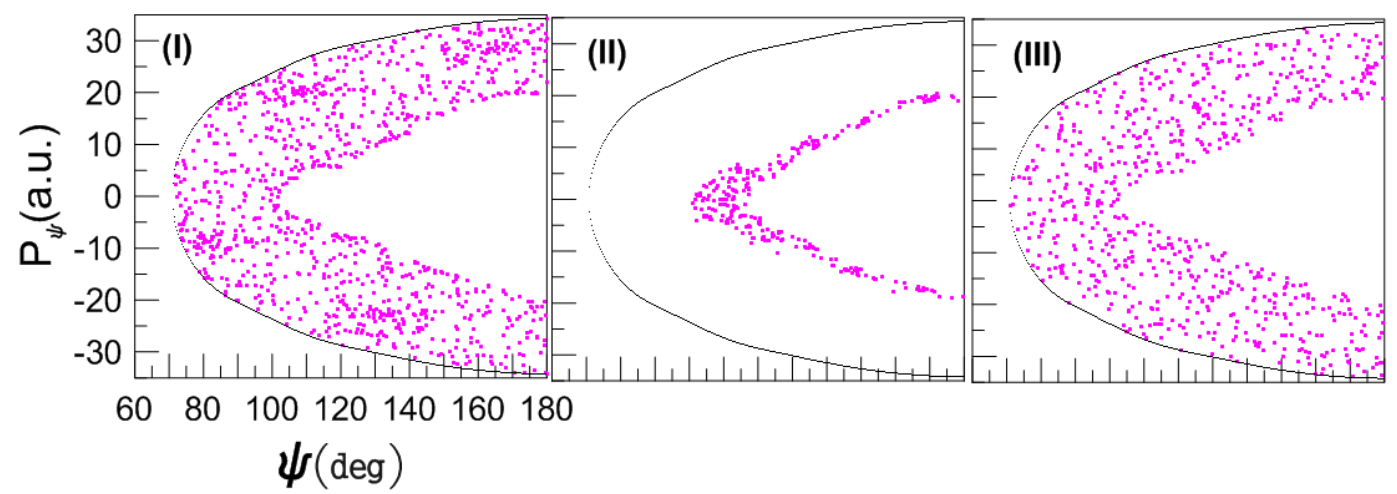

Figura 5.7: Influencia del cantoro existiendo a $E=2964,7 \mathrm{~cm}^{-1}$, que actúa como una barrera parcial para el flujo de trayectorias en el espacio de fases, esto implica que una trayectoria visita de forma alternada las regiones exteriores e interiores con diferentes características dinámicas. Este efecto es responsable de los cambios de pendiente encontrados en la correspondiente evolución $J$ v.s. $s$ representado en Fig. 5.6 
local) y aumenta suavemente en los laterales (discutido en secciones previas) formando una especie de figura en M irregular. Este comportamiento es más importante en la mitad y parte izquierda de la curva, y podría entenderse como un método para localizar las correspondientes órbitas periódicas. Si consideramos el panel intermedio correspondiente al nivel de energía $E=2964,5 \mathrm{~cm}^{-1}$ tenemos el mismo comportamiento en las regiones regulares, esto es, en el extremo derecho de la curva correspondiente al isómero LiNC y también la parte nueva del extremo izquierdo correspondiente al isómero LiCN. En este último la forma de M correspondiente a la resonancia $1: 4$ es claramente visible. Sin embargo, a esta energía tenemos una región grande de comportamiento caótico, donde el $S G I$ es mucho mayor y aparece de forma discontinua debido a las grandes oscilaciones.

Finalmente en el último panel de la derecha consideramos la mayor energía $E=3595,6 \mathrm{~cm}^{-1}$, que se corresponde con la mayor región caótica observada, incluyendo la región asociada al punto hiperbólico en la superficie de energía potencial separando los dos isómeros. Los dos pozos de potencial están conectados a esta energía, lo que supone la aparición de trayectorias isomerizantes. Como resultado, la dinámica es mucho más caótica, particularmente en esta última región, así los valores del $S G I$ son mayores. Realmente se pueden distinguir dos partes en la región caótica, con diferentes valores del indicador. En la parte izquierda localizada más allá del punto de silla de la superficie de energía potencial, donde la dinámica es más irregular debido a la presencia del correspondiente punto fijo inestable, el $S G I$ es mayor que en la parte de la derecha, más próximo al isómero LiNC, que tiene una dinámica regular.

\subsubsection{Conclusiones de la sección}

En esta sección, hemos estudiado la dinámica vibracional del sistema de isomerización $\mathrm{LiNC} / \mathrm{LiCN}$ descrito por un modelo realista 2D dimensional desde la perspectiva geometrodinámica, estudiando la estabilidad de ciertas trayectorias representativas del sistema para diferentes valores de la energía de excitación.

Igualmente hemos definido un indicador geométrico de estabilidad SGI que nos ha permitido diferenciar claramente entre órbitas regulares y caóticas. En las primeras, el $S G I$ es continuo y acotado, mientras que para las últimas los valores para el $S G I$ es discontinuo y no acotado.

Este indicador $S G I$ puede ser usado para calcular eficientemente órbitas periódicas, ya que presenta mínimos locales alrededor de las cadenas de islas 
asociadas.

Finalmente, remarcamos que el formalismo geometrodinámico aquí presentado es totalmente intrínseco, siendo además original y adecuado para el estudio de sistemas dinámicos realistas. Es más, se puede extender fácilmente y de forma natural al estudio de otros sistemas realistas similares de más de dos dimensiones, campo que permanece inexplorado a pesar de su interés e importancia.

\subsection{Convexidad de la frontera de la región de Hill e inestabilidad}

Para caracterizar la dinámica del sistema es importante las condiciones iniciales a la hora de integrar las trayectorias.

Todas las trayectorias que hemos integrado alcanzan aproximadamente la frontera de la región de Hill en algún momento. Este hecho motiva el estudio de la dinámica de nuestro sistema molecular a través de trayectorias con condiciones iniciales en dicha frontera.

Para ello hemos calculado el valor del $S G I$ para tiempos cortos de trayectorias con condiciones iniciales $\left(\theta_{0}, R_{0}\right)$ a lo largo de la frontera $V\left(\theta_{0}, R_{0}\right)=E$, es decir, $p_{R}=0$ y $p_{\theta}=0$ (energía cinética nula) con un valor para la energía de $E=1500,5 \mathrm{~cm}^{-1}$.

La curvatura es singular en estos puntos de modo que los cálculos han sido realizados para una energía ligeramente mayor $\hat{E}$ de valor $\hat{E}=E \times\left(1+10^{-13}\right)$ obteniendo los resultados representados en la Figura 5.8.

Las trayectorias estables y caóticas para el intervalo $1,7<\theta<2,1$ rad para la región superior de la frontera (color rojo en el gráfico de la parte superior izquierda en la Fig. 5.8) son representadas en la Fig. 5.9 desde (1) hasta (8).

Las trayectorias periódicas estables se corresponden con mínimos locales del $S G I$, en particular los puntos (1), (2), (3), (5) y (7), y las trayectorias caóticas con (4) and (8).

La trayectoria representada por (7) es la trayectoria más estable del sistema delacionada con el toro KAM con la relación de frecuencias más irracional, siendo este toro el último roto en la transición de orden a caos. 
Las trayectorias correspondientes en $(\theta, R)$ están representadas en los gráficos superiores de la Fig. 5.9.

Las cadenas de islas se representan como aquellas formando valles en el diagrama global de SGI, la estabilidad de la órbita periódica resonante en el mínimo se indica a través de la anchura del valle como se ilustra en las Secciones de Poincaré en la Fig. 5.10 .

Para localizar una órbita caótica del diagrama de $S G I$ global buscamos puntos hiperbólicos localizados entre las cadenas de islas (Teorema de Poincaré Birkchoff) de modo que consideramos condiciones iniciales en los extremos de los valles previos indicados por mínimos locales por (4) y (8) en la Figura 5.9.

Las Secciones de Poincaré de todas estas trayectorias se ilustran en la Figura 5.10 .

Calculamos el indicador $S G I$ a lo largo de trayectorias con condiciones ini-

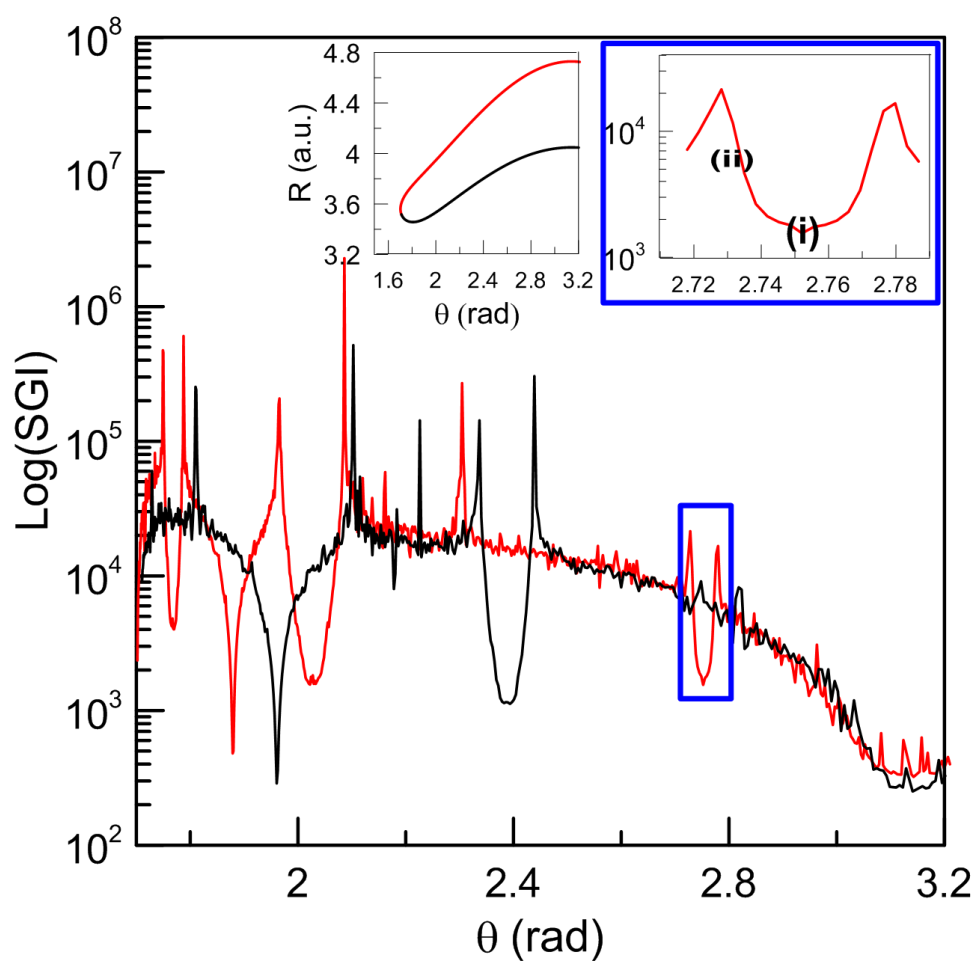

Figura 5.8: Valores de $S G I$ para diferentes trayectorias con condiciones iniciales a lo largo de la frontera de la región de Hill (gráfico superior izquierdo) para la energía, $E=1500,5 \mathrm{~cm}^{-1}$, el intervalos $2,71<\theta<2,79$ ) rad de la parte superior (rojo) es ampliada en los lugares donde una trayectoria periódica estable (i) y otra de la cadena de islas (ii) han sido señaladas.

ciales en la frontera para energía $E=500 \mathrm{~cm}^{-1}$ localizando una trayectoria 

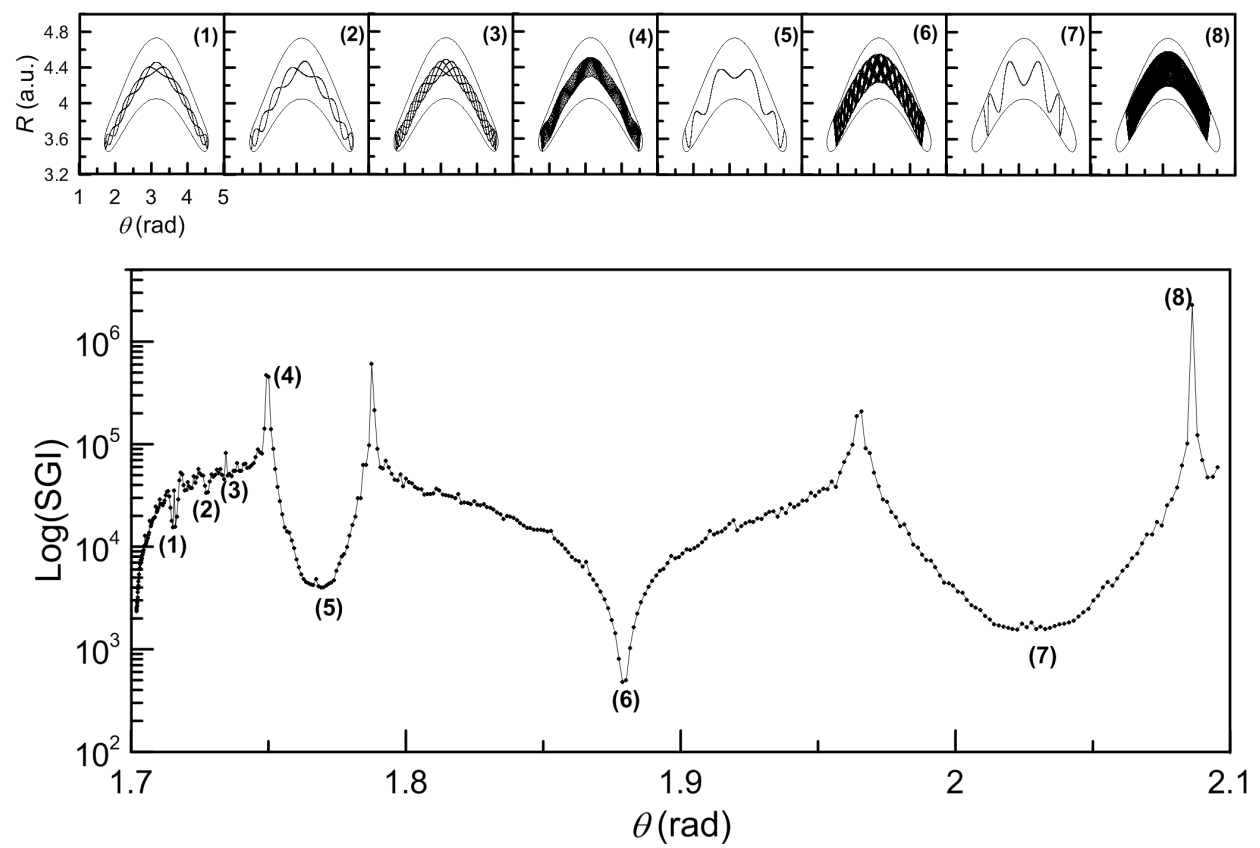

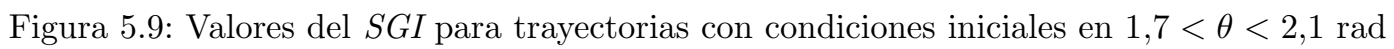
para la parte superior de la frontera (color rojo en el gráfico superior izquierdo en la Fig. (5.8), a la energía $E=1500,5 \mathrm{~cm}^{-1}$. Trayectorias periódicas estables están indicadas como (1), (2), (3), (5), (7) y trayectorias caóticas como (4) and (8). La trayectoria cuasiperiódica (7) es la más estable del sistema. Las trayectorias correspondientes en $(\theta, R)$ son representadas in los gráficos superiores.

caótica y una periódica representadass por los puntos máximo y mńimo en el gráfico para SGI en la Figura 5.11. Las Secciones de Poincaré están representadas en la Figura 5.12 . 


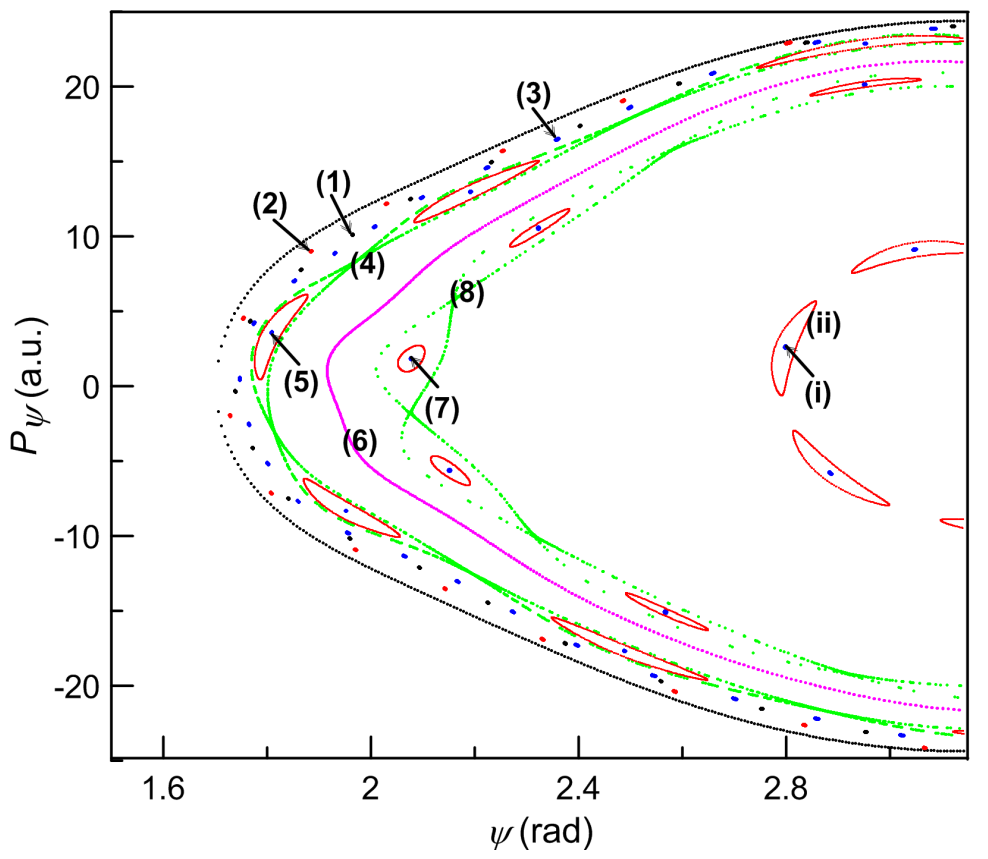

Figura 5.10: Secciones de Poincaré para todas las trayectorias representadas en las Fig. 5.8 y 5.9 para energía $E=1500,5 \mathrm{~cm}^{-1}$. La curva negra representa la frontera de la región accesible en el espacio de fases.

\subsection{Indicador $S G I$ e inestabilidad}

La frontera de la región de Hill y particularmente el signo de su curvatura está directamente relacionado con la estabilidad de trayectorias que alcanzan dichos puntos (rebotan en ellos).

En sistemas con curvatura Gaussiana positiva en todos los puntos dentro de la región de Hill es bastante natural considerar las regiones no convexas (curvatura negativa) de la frontera como fuente de inestabilidades para trayectorias que rebotan en ellas.

En este sentido podríamos decir que las trayectorias regulares deben rebotar o tocar solamente regiones convexas (curvatura positiva) de la frontera de la región de Hill así como las trayectorias caóticas deberían alcanzar zonas no convexas al menos una vez Saa, 2004.

Nosotros mostramos que esta afirmación no es cierta en general encontrando una trayectoria periódica estable que rebota en una región no covexa (curvatura negativa) de la frontera y otra trayectoria caótica restringida a regiones convexas de esta misma frontera. 


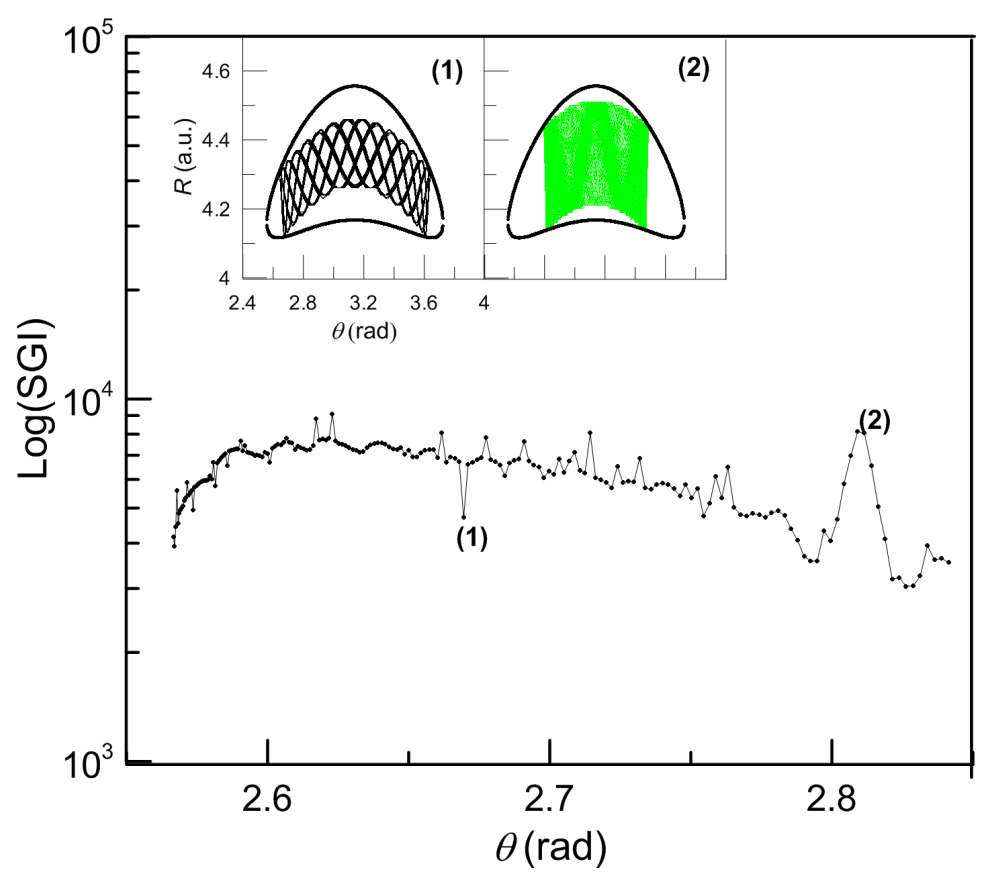

Figura 5.11: Valores de SGI para trayectorias con condiciones iniciales en $2,5<\theta<2,9$ rad a la energía $E=500 \mathrm{~cm}^{-1}$. Marcamos una trayectoria periódica estable por (1) y una caótica por (2). Las correspondientes trayectorias en $(\theta, R)$ se representan en los gráficos superiores.

La curvatura $\kappa$ de la frontera de la región de Hill considerada como el conjunto de nivel $\{(R, \theta) / V(R, \theta)=E\}$ se puede calcular mediante

$$
\kappa=-\frac{\left(\frac{\partial V}{\partial \theta}\right)^{2} \frac{\partial^{2} V}{\partial R^{2}}-2 \frac{\partial^{2} V}{\partial R \partial \theta} \frac{\partial V}{\partial R} \frac{\partial V}{\partial \theta}+\frac{\partial^{2} V}{\partial \theta^{2}}\left(\frac{\partial V}{\partial R}\right)^{2}}{\left(\left(\frac{\partial V}{\partial R}\right)^{2}+\left(\frac{\partial V}{\partial \theta}\right)^{2}\right)^{\frac{3}{2}}}
$$

Estudiamos la convexidad para diferentes conjuntos de nivel $\left\{V(R, \theta)=E / E \in(0,1510) \mathrm{cm}^{-1}\right\}$ y dibujamos los puntos de curvatura cero (cambio de convexidad) como la línea roja en la Fig. 5.13, los puntos no convexos en la región inferior de la frontera para la energía $E=1500,5 \mathrm{~cm}^{-1}$ están en el intervalo $2,16<\theta<4,13 \mathrm{rad}$.

Las dos trayectorias estudiadas con esta energía se indican como (i) (regular) y (4) (caótica) en la Fig. 5.10 donde se representan sus correspondientes PSS.

En la Fig. 5.13 representamos estas dos trayectorias in $(\theta, R)$ y la curvatura cero para el correspondiente conjunto de nivel.

La trayectoria caótica solamente toca la región convexa de la frontera mientras 


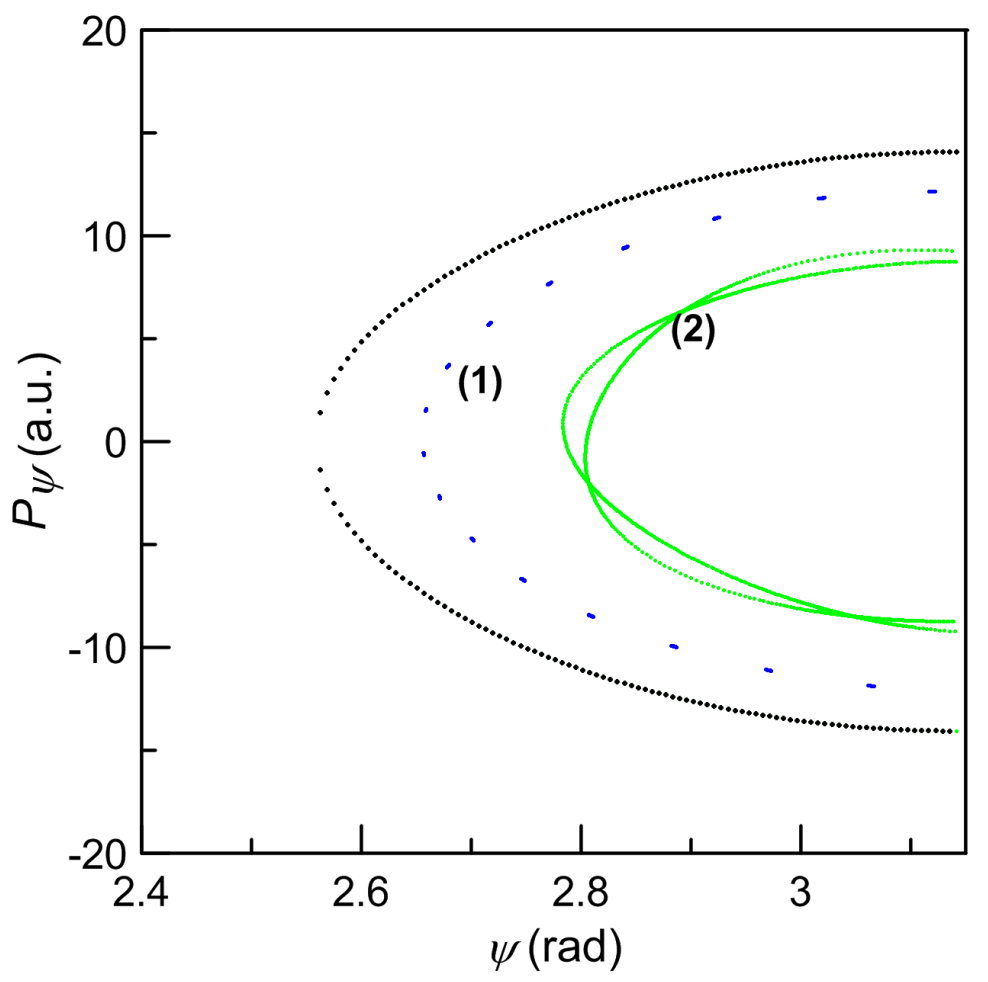

Figura 5.12: Secciones de Poincaré para las dos trayectorias representadas en la Fig. 5.11 a la energía $E=500 \mathrm{~cm}^{-1}$. La curva negra representa la frontera de la región accesible en el espacio de fases.

que la regular entra en contacto con punto no convexos. El comportamiento de la trayectoria caótica viene a apoyar la idea de que la fuente de caos es la resonancia paramétrica, pero la trayectoria regular motiva la idea de un mecanismo de estabilización adicional.

Cuando definimos la métrica de Jacobi $g_{i j}(R, \theta)$ es bastante frecuente interpretar que la energía potencial curva el espacio subyacente (con tensor de energía cinética $a_{i j}(R, \theta)$ como métrica), en el caso particular de un espacio subyacente de curvatura cero (usualmente $a_{i j}=\delta_{i j}$ ) el primer término del segundo miembro de (5.13) es cero y la curvatura está causada por la energía potencial.

En nuestro sistema molecular el tensor de energía cinética $a_{i j}(R, \theta)$ define un espacio subyacente curvado positivamente, por tanto aunque la energía potencial fuera eliminada el espacio sería igualmente curvado positivamente.

Teniendo en cuenta que la estabilidad está gobernada por la ecuación de JLC donde $\hat{R}$ incluye la curvatura del espacio subyacente $\tilde{R}$ podemos deducir el correspondiente efecto estabilizador de esta última curvatura en la dinámica del sistema. 

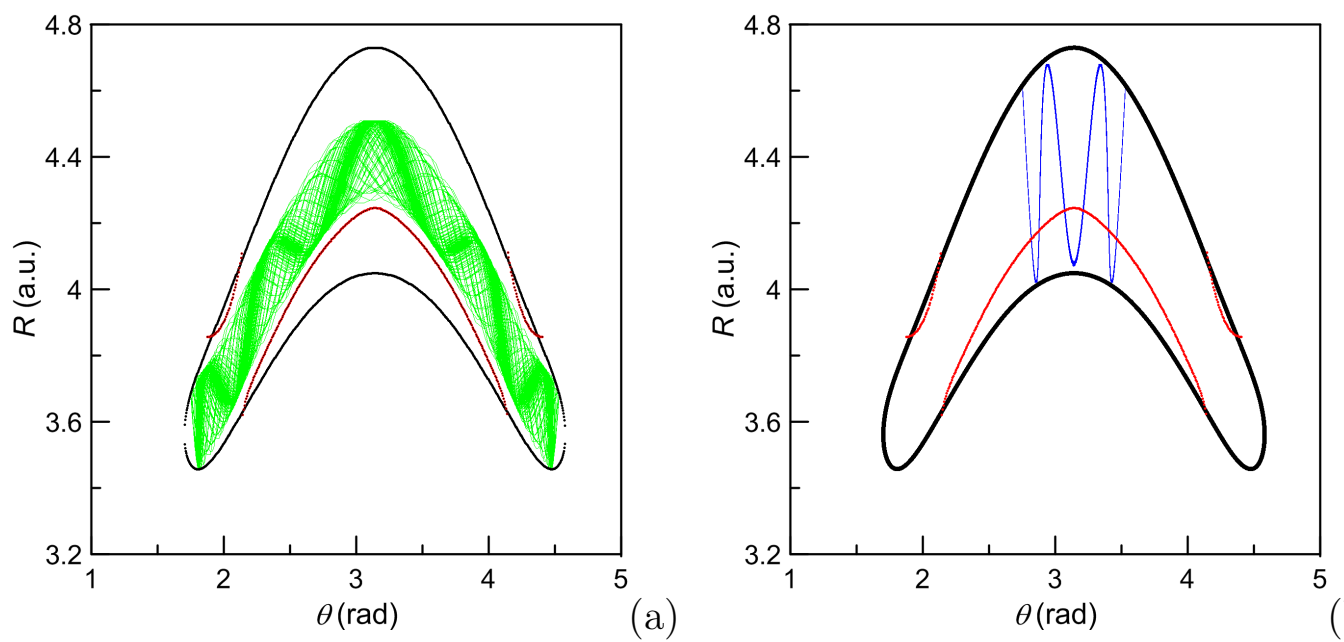

Figura 5.13: Una trayectoria caótica (a) restringida a puntos convexos de la frontera de la región de Hill y una trayectoria periódica estable (b) alcanzando la frontera en puntos no convexos a una energía de $E=1500,5 \mathrm{~cm}^{-1}$. Estas trayectorias se corresponden a (4) y (i) respectivamente en la Figura 5.10 .

\subsubsection{Conclusiones de la sección}

Hemos estudiado el sistema realista de isomerización LiNC/LiCN en dos grados de libertad desde una perspectiva geometrodinámica.

Hemos considerado la región de Hill como un conjunto suficiente de condiciones iniciales para caracterizar la dinámica del sistema.

Habieno definido previamente el indicador SGI hemos calculado su valor a lo largo de trayectorias durante un corto periodo de tiempo donde las condiciones iniciales han sido tomadas de la frontera de la región de Hill localizando órbitas periódicas estables como mínimos de este indicador, igualmente consideramos la anchura de dicho valle como una medida de su estabilidad.

Igualmente hemos localizado trayectorias caóticas como los máximos locales alrededor de los valles antes citados justo en los extremos de la correspondiente región de estabilidad.

Hemos estudiado la importancia de la curvatura de la región de Hill para la estabilidad de las trayectorias, observando que las trayectorias estables de 
nuestro sistema se encuentran localizadas principalmente para valores $\theta<2,16$ rad y $\theta>4,13 \mathrm{rad}$ para el intervalo $\theta \in[0,2 \pi]$ que se corresponde con las regiones de mayor convexidad de la frontera apoyando la idea de reducir nuestro sistema a un problema de billar clásico. Sin embargo encontramos una trayectoria periódica estable con puntos de retorno en punto no convexos de la región de Hill. Por otro lado también encontramos una trayectoria caótica dentro de la supuesta 'región de estabilidad' tocando únicamente en puntos convexos de la frontera.

Por tanto deducimos que el estudio de la estabilidad de trayectorias en nuestro sistema molecular no puede ser reducido a un problema clásico de billar a pesar de que la curvatura gaussiana es positiva en todos los puntos dentro de la región de Hill.

Este resultado vienen a apoyar la hipótesis de la resonancia paramétrica como fuente principal de aparición de caos en el caso de las trayectorias caóticas. Sin embargo en el caso de trayectorias regulares rebotando en puntos no convexos de la frontera entendemos que es la underlying curvatura $\tilde{R}$ la responsable de esta falta de comportamiento inestable.

Este mecanismo de estabilización a través de la curvatura escalar de la variedad subyacente sigue siendo estudiado en nuestro grupo. 


\section{Capítulo 6}

\section{Condición catenaria: indicador de caos}

\subsection{Fundamento teórico}

En esta sección proponemos un nuevo indicador de caos basado en el análisis de la fluctuación de un observable definido previamente en la variedad riemanniana dotada con la métrica de Jacobi (mechanical manifold (MM)) basado en la analogía existente entre Mecánica y Estática y analizaremos su aplicación en el caso particular del sistema hamiltoniano bidimensional de Hénon-Heiles Henon et Heiles, 1964 como ilustración, así como a otros sistemas hamiltonianos tridimensionales.

La aplicación sistemática a más de dos grados de libertad desde el punto de vista del cálculo numérico es mucho más costosa y menos novedosa, lo que nos ha llevado a investigar los sistemas hamiltonianos de tres grados de libertad desde la perspectiva de la integrabilidad, encontrando una condición de suficiencia para la integrabilidad de este tipo de sistemas.

La idea, ya planteada en el pasado por Johann Bernoulli Bernoulli, 1742, propone que el problema de encontrar la trayectoria de una partícula conectando dos puntos en el espacio de configuración pueda ser reformulado en términos de mecánica del equilibrio como un mínimo de la energía potencial de una cuerda flexible pero no elástica sometida a cierta tensión.

Es más, esta analogía justifica que el principio mecánico de acción estacionaria 
Tabla 6.1: Equivalencia entre integrales de acción para la Mecánica Clásica y la Estática.

\begin{tabular}{ll}
\hline \hline Partícula (Mecánica) & Cuerda no elástica (Estática) \\
\hline$\delta \int m v d \ell=0$ & $\delta \int T d \ell \underset{\substack{T-V=\text { const } \\
=}}{\delta} \delta V d \ell=0$ \\
\hline
\end{tabular}

para una particula sea equivalente al equilibrio estático en mecánica Static Mechanical Equilibrium(SME) para una una cuerda flexible y no elástica sometida a un campo gravitatorio uniforme, como se muestra con las correspondientes expresiones matemáticas en la siguiente tabla donde $m v$ es el momento lineal de la partícula, $T$ la tensión en la cuerda, $V$ la energía potencial y $d s$ un diferencial de longitud. Como se indica en la segunda columna de la tabla, el principio de acción estacionaria para el SME de la cuerda es equivalente al principio de trabajos virtuales Virtual Work Principle (VWP) para el SME con fuerzas derivadas de un potencial $V$, relacionado directamente con un equilibrio estable, pudiéndose deducir este principio a través del formalismo geometrodinámico.

Realmente, en el formalismo geométrico es posible escribir las ecuaciones para la cuerda colgante (curva catenaria) como las ecuaciones de las geodésicas en cierta variedad riemanniana obtenida por transformación conforme de la métrica euclídea, siendo la tensión $T$ el factor conforme correspondiente Meckler, 1976.

El objetivo de esta sección es estudiar la estabilidad de trayectorias en un sistema dinámico conservativo a través del análisis del equilibrio estático previo como hemos indicado en la segunda columna de la tabla ( refTable:III) para las correspondientes trayectorias, como si estas fueran cuerdas colgantes flexibles y no elásticas en la MM descrita previamente.

Este proceso lo llevaremos a cabo en tres pasos:

Primero, geometrizaremos la dinámica de nuestro sistema a través del formalismo geometrodinámico, construyendo una variedad riemanniana donde las trayectorias del sistema se corresponderán con geodésicas de esta variedad. Con este fin utilizaremos la MM derivada de la métrica Jacobi.

Segundo, en la MM previamente determinada, definiremos una nueva función potencial $V(q)$ en cada punto y entonces aplicaremos el VWP para SME en esta nueva MM. Mostramos que aplicar este principio es equivalente al cálculo de geodésicas en la variedad riemanniana obtenida como la transformación de Weyl para la métrica de Jacobi en MM, deduciendo un nuevo conjunto de relaciones métricas equivalentes a la aplicación del VWP. 
Observamos que en la MM inicial (antes de incluir la función potencial) no hay fuerzas reales si la energía es constante, el tiempo físico $t$ se reemplaza por la longitud de arco de las geodésicas $s$ y la velocidad es unitaria.

De acuerdo con esto, las únicas fuerzas son aquellas ficticias o inerciales (ya que vienen de los símbolos de Christoffel definidos por la variedad).

Las fuerzas ficticias pueden ser eliminadas localmente utilizando coordenadas normales, cancelando así los símbolos de Christoffel. En estas nuevas coordenadas, las ecuaciones geodésicas se corresponden con líneas rectas en $\mathbb{R}^{n}$.

Una vez que las fuerzas inerciales han sido eliminadas localmente y añadimos una función potencial, podemos pensar (localmente) acerca de las misma fuerzas derivando del gradiente de esta función potencial pero sin fuerzas de inercia.

Este planteamiento está basado en argumentos Quapp et Heidrich, 1984 similares a aquellos utilizados por Fukui [Fukui, 1970] en el contexto de la reactividad química cuando define las intrinsic reaction coordinates curves en la búsqueda por los caminos de mínima energía (MEP) conectando reactivos y productos en la teoría del estado de transición Eyring et Polanyi, 1931. Esta interpretación está conectada con el string method para el cálculo de MEPs Ren et Vanden-Eijnden, 2002, Weinan et al., 2007.

Tercero, finalmente el observable adecuado se define como el potencial acumulado $\int V d s$ a lo largo de las diferentes trayectorias $\gamma(s)$ con longitud constante (como en el caso de la curva catenaria) en la MM. A continuación analizamos sus fluctuaciones como un indicador para estabilidad y caos, en el sentido que mayor fluctuaciones indican más inestabilidad, mientras que menores fluctuaciones se corresponden con trayectorias estables.

En esta sección presentaremos el formalismo geometrodinámico y una generalización del VWP para la métrica Jacobi. A continuación definiremos el indicador de caos con aplicaciones al modelo Hénon-Heiles. Después conectaremos el concepto de simetría con Campos Vectoriales Killing y constantes de movimiento. Tras esto, generalizamos las ideas previas a sistemas tres dimensionales encontrando de manera novedosa una condición suficiente de integrabilidad. Comprobamos esta condición suficiente aplicńdola a sistemas integrables bien conocidos. 


\subsection{Principio de Trabajos Virtuales Geometrodinámico}

El VWP afirma que para un sistema mecánico en equilibrio estático el trabajo virtual llevado acabo por las fuerzas en un desplazamiento virtual compatible con las constraints es nulo.

Comenzando de coordenadas generalizadas $\left\{q^{i}\right\}$, si realizamos una transformación $\mathbf{r}=\mathbf{r}\left(q^{i}\right)$, la correspondiente velocidad se expresa como $\mathbf{v}=d \mathbf{r} / d t=$ $\left(\partial \mathbf{r} / \partial q^{i}\right) \dot{q}^{i}$ y los desplazamientos virtuales vienen dados por $\delta \mathbf{r}=\left(\partial \mathbf{r} / \partial q^{i}\right) \delta q^{i}$, tal que el trabajo virtual asociado con las fuerzas aplicadas $\mathbf{F}_{i}$ es descrito como

$$
\delta W=\mathbf{F} \cdot \delta \mathbf{r}=\mathbf{F} \cdot \frac{\partial \mathbf{r}}{\partial q^{i}} \delta q^{i}=\mathbf{Q}_{i} \delta q^{i}=0,
$$

siendo $\mathbf{Q}_{i}$ las fuerzas generalizadas.

Para fuerzas aplicadas derivadas de un potencial, es decir, $\mathbf{F}=-\nabla V, \mathrm{y}$ ligaduras holonómicas, el VWP se escribe como sigue

$$
\delta W=\mathbf{F} \cdot \delta \mathbf{r}=-\frac{\partial V}{\partial q^{i}} \delta q^{i}=-\delta V=0 .
$$

Como consecuencia una posición de equilibrio estable $x_{s}$ se corresponde con un mínimo para la función de energía potencial $V$. Este resultado se puede extender a espacios con un número infinito de dimensiones (espacios funcionales) utilizando métodos variacionales, llegando a la conclusión de que las curvas con las correspondientes ligaduras son aquellas donde la energía potencial total a lo largo de ellas es estacionaria, esto es,

$$
\delta \int V d \ell=0
$$

En la próxima sección ilustramos esta idea considerando el caso del arco más estable en arquitectura, ver Fig. (6.3) que resulta tener la misma forma que la curva catenaria invertida, y estudiaremos su estabilidad desde el punto de vista geometrodinámico.

\subsection{La curva catenaria: paradigma de estabilidad}

Consideramos ahora el problema de la curva catenaria, estudiada desde una perspectiva geometrodinámica. 


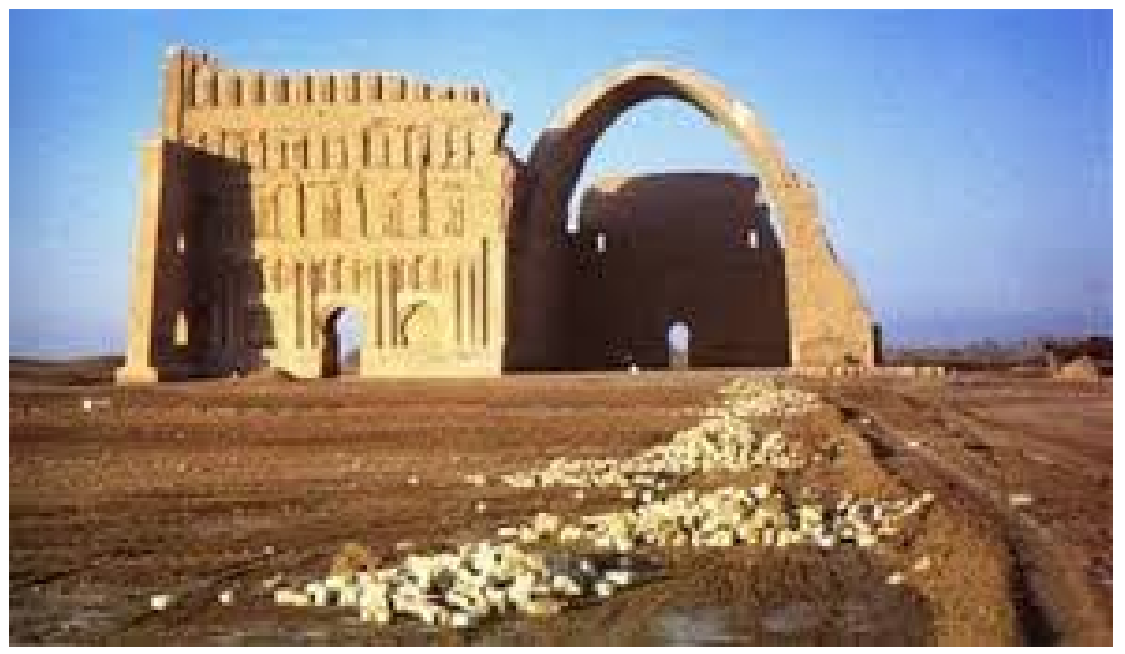

Figura 6.1: Palacio de Ctesifonte o arco de Tak Kisra en Baghdag, próximo al Tigris, construido III ac. El arco tiene $30 \mathrm{~m}$ de alto y $25 \mathrm{~m}$ ancho e ilustra el concepto de estabilidad, i.e. la forma del arco es una catenaria invertida.

Deduciremos las correspondientes ecuaciones para la curva y la condición VWP como una condición de estabilidad.

Las ecuaciones diferenciales que describen la curva para una cuerda flexible y no elástica en un campo de fuerzas genérico puede ser escrito en coordenadas generales $\left\{e_{i}\right\}$ como Rojo, 2005

$$
\frac{d T_{i}}{d s}+\frac{\partial \mathcal{G}^{j k}}{\partial q^{i}} \frac{T_{j} T_{k}}{2 T}=\frac{\partial T}{\partial q^{i}}
$$

donde $T(\mathbf{q})$ es la tensión de la cuerda, y

$$
T_{i}=\left\langle T \frac{d q^{j}}{d s} \mathbf{e}_{j}, \mathbf{e}_{i}\right\rangle=T \frac{d q^{j}}{d s}\left\langle\mathbf{e}_{j}, \mathbf{e}_{i}\right\rangle=T \frac{d q^{j}}{d s} \mathcal{G}_{j i}
$$

donde $\langle\cdot, \cdot\rangle$ es e producto escalar derivado dell tensor métrico $\mathcal{G}_{j i}, \mathrm{y} d q^{j} / d s$ es la $j$-ésima componente del vector tangente unitario a la curva.

En coordenadas cartesianas $\mathcal{G}_{j i}=\delta_{j i}$. Entonces la ecuación (6.4) se reduce a

$$
\frac{d}{d s}\left(T \frac{d\left(q^{j} \delta_{j i}\right)}{d s}\right)=\frac{\partial T}{\partial q^{i}}
$$

De la mecánica de Newton, sabemos que el segundo miembro de esta ecuación representa la componente de una fuerza por unidad de longitud. En el caso de un sistema bidimensional con $\mathbf{q}=(x, y)$ y una fuerza vertical le corresponde 
un equilibrio estático dado por

$$
\begin{aligned}
\frac{d}{d s}\left(T \frac{d x}{d s}\right) & =0 \quad \longrightarrow \frac{d}{d s}(T \cos \theta)=0 \\
\frac{d}{d s}\left(T \frac{d y}{d s}\right) & =-f_{y} \longrightarrow \frac{d}{d s}(T \sin \theta)=-f_{y}
\end{aligned}
$$

de la que podemos obtener las ecuaciones para la curva catenaria.

Equivalentemente, podemos obteber el mismo resultado aplicando el VWP al SME en $\mathbb{R}^{2}$

$$
\delta \int V d \ell=\delta \int_{x_{i}}^{x_{f}} y(x) \sqrt{1+\left(y^{\prime}(x)\right)^{2}} d x
$$

con la ligadura de longitud constante y con $V(y)=m g y$. Todos estos bien conocidos resultados se pueden obtener igualmente a partir de la versión geometrodinámica haciendo una transformación conforme a la métrica euclídea $\delta_{i j}$ obteniendo una variedad riemanniana con una nueva métrica que en coordenadas tiene la forma

$$
h_{i j}=T^{2} \delta_{i j}
$$

En este caso, la longitud de arco es $d \bar{s}=\sqrt{T^{2} \delta_{i j} d q^{i} d q^{j}}=T d \ell$, con $d \ell$ la longitud de arco euclídea. Como dijimos, trayectorias son geodesicas en esta nueva métrica, y las ecuaciones para esta última pueden ser derivadas del principio de acción estacionaria para la longitud de arco

$$
\delta \int d \bar{s}=0
$$

En coordenadas locales estas escuaciones son

$$
\begin{aligned}
& \frac{d^{2} q^{i}}{d \bar{s}^{2}}+\Gamma_{j k}^{i} \frac{d q^{j}}{d \bar{s}} \frac{d q^{k}}{d \bar{s}}= \\
& \frac{d^{2} q^{i}}{d \bar{s}^{2}}+\frac{1}{2} h^{i m}\left(\partial_{j} h_{m k}+\partial_{k} h_{m j}-\partial_{m} h_{h k}\right) \frac{d q^{j}}{d \bar{s}} \frac{d q^{k}}{d \bar{s}}=0,
\end{aligned}
$$

donde $\Gamma_{j k}^{i}$ son los símbolos de Christoffel para la métrica $h_{i j}$. Las ecuaciones de la curva son las ecuaciones geodésicas para la métrica $h_{i j}$, con una longitud de arco $d \bar{s}=\sqrt{h_{i j} d q^{i} d q^{j}}$, podemos aplicar la propiedad de extremo y obtener

$$
\begin{aligned}
\delta \int d \bar{s} & =\delta \int \sqrt{h_{i j} d q^{i} d q^{j}}=\delta \int T \sqrt{\delta_{i j} d q^{i} d q^{j}} \\
& =\delta \int T d \ell \underset{T-V=\text { const }}{=} \delta \int V d \ell=0,
\end{aligned}
$$

con la restricción de que la longitud de la cuerda sea constante.

La última expresión se refiere al VWP en $\mathbb{R}^{2}$ para una fuerza derivada de un 
potencial obtenida a partir de la versión geometrodinámica. Concluimos entonces que las ecuaciones de las geodésicas en la métrica $h_{i j}$ son equivalentes a las ecuaciones para las trayectorias estables del sistema. En esta versión geometrodinámica la propiedad de geodésica (6.10) se usa para obtener trayectorias cumpliendo VWP, lo que muestra el carácter fundamental del formalismo geometrodinámico.

\subsubsection{La hipótesis}

Consideramos las trayectorias para nuestros sistemas dinámicos como si fueran cuerdas de longitud constante en una MM. En la sección previa la métrica utilizada fue la euclídea $\delta_{i j}$. Ahora consideraremos la posibilidad de generalizr el VWP (GVWP)a métricas no euclídeas. Habíamos visto como las ecuaciones de la curva catenaria pueden obtenerse resolviendo la expresión $\delta \int V d \ell=0$, siendo $d \ell$ la longitud de arco para la métrica euclídea $\delta_{i j}$, y donde la única fuerza actuando se derivaba de un potencial, a saber, resolviendo un problema de Esática.

Ahora aplicaremos esta idea a sistemas dinámicos generales, donde en lugar de tener una métrica euclídea $\delta_{i j}$ usaremos la métrica de Jacobi $\mathcal{G}_{\mu \nu}(\mathbf{q})=$ $2(E-V(\mathbf{q})) a_{\mu \nu}(\mathbf{q})$ definida para un sistema dinámico particularcon la correspondiente longitud de arco $d s=2(E-V(\mathbf{q})) d t$. Entonces nuestra hipótesis es que la trayectoria más estable $\gamma(t)$ del sistema cumplirá la siguiente propiedad

$$
\delta \int_{\gamma(t)} V d s=0
$$

con la restricción de longitud constante $L$, es decir, $\int_{0}^{s} d s=2 \int_{0}^{t}(E-V(\mathbf{q}(t))) d t=$ $L$. Si éste es el caso, podríamos analizar la estructura del espacio de fases considerando el comportamiento del observable $\int_{\gamma(t)} V d s$ para trayectorias con diferentes condiciones iniciales para la misma energía $E$. Es importante recalcar que el tiempo de integración $t$ en ecuación (6.13) es diferente para cada trayectoria, dado que la longitud constante $L$ está previamente definida en la MM, y entonces cada trayectoria necesita un intervalo de tiempo físico diferente $t$ para alcanzar la longitud total $L$. 


\subsection{Geometrodinámica y generalización del Teorema de Trabajos Virtuales}

El objetivo de esta sección es desarrollar el GVWP para sistemas conservativos hamiltonianos en espacios euclídeos a espacios riemannianos utilizando la formulación geometrodinámica. Para esto, empezamos con la métrica de Jacobi previamente definida

$$
\mathcal{G}_{\mu \nu}(\mathbf{q})=2(E-V(\mathbf{q})) a_{\mu \nu}(\mathbf{q})
$$

donde $a_{\mu \nu}(\mathbf{q})$ is la matriz de masa, y las ecuaciones geodésicas para $\mathcal{G}_{\mu \nu}$ son

$$
\frac{d^{2} q^{\mu}}{d s^{2}}+\Gamma_{\lambda \nu}^{\mu} \frac{d q^{\lambda}}{d s} \frac{d q^{\lambda}}{d s}=0
$$

donde $\Gamma_{\lambda \nu}^{\mu}$ son los símbolos de Christoffel para la métrica $\mathcal{G}_{\mu \nu}$. Ahora llevamos a cabo la transformación conforme conocida como transformaciónN de Weyl,

$$
\overline{\mathcal{G}_{\mu \nu}}(\mathbf{q})=e^{2 \Omega(\mathbf{q})} \mathcal{G}_{\mu \nu}(\mathbf{q})
$$

con longitud de arco para la nueva métrica

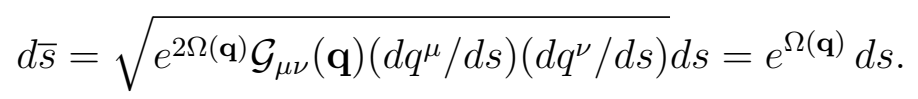

A continuación hacemos $\Omega(\mathbf{q})=\ln V(\mathbf{q})$, y tenemos el principio variacional como

$$
\delta \int d \bar{s}=\delta \int e^{\Omega(\mathbf{q})} d s=\delta \int e^{\ln V(\mathbf{q})} d s=\delta \int V(\mathbf{q}) d s=0 .
$$

Esto es, las geodésicas para la métrica $\overline{\mathcal{G}_{\mu \nu}}$ son aquellas trayectorias en la métrica de Jacobi que son extremos para la enrgía potencial a lo largo de ellas, equivalente al VWP para fuerzas derivadas de la función de energía potencial $V$ en la MM. Las trayectorias para la nueva métrica $\overline{\mathcal{G}_{\mu \nu}}(\mathbf{q})=e^{2 \Omega(\mathbf{q})} \mathcal{G}_{\mu \nu}(\mathbf{q})$ están definidas como

$$
\frac{d^{2} q^{\mu}}{d \bar{s}^{2}}+\overline{\Gamma_{\lambda \nu}^{\mu}} \frac{d q^{\lambda}}{d \bar{s}} \frac{d q^{\nu}}{d \bar{s}}=0
$$

donde $\overline{\Gamma_{\lambda \nu}^{\mu}}=\Gamma_{\lambda \nu}^{\mu}+\mathcal{G}^{\lambda \sigma}\left(\mathcal{G}_{\sigma \nu} \partial_{\mu} \Omega+\mathcal{G}_{\mu \sigma} \partial_{\nu} \Omega-\mathcal{G}_{\mu \nu} \partial_{\sigma} \Omega\right)$ representan los nuevos símbolos de Christoffel. Teniendo en cuenta que $d \bar{s}=e^{\Omega(\mathbf{q})} d s \rightarrow d s / d \bar{s}=$ $e^{-\Omega(\mathbf{q})}$ tal que $d q^{\mu} / d \bar{s}=\left(d q^{\mu} / d s\right)(d s / d \bar{s})=\left(d q^{\mu} / d s\right) e^{-\Omega(\mathbf{q})}$ y $d^{2} q^{\mu} / d \bar{s}^{2}=$ $(d / d \bar{s})\left(d q^{\mu} / d \bar{s}\right)=e^{-2 \Omega(\mathbf{q})}\left(\left(d^{2} q^{\mu} / d s^{2}\right)-\left(d q^{\mu} / d s\right)(d \Omega / d s)\right)$ ecuación 6.18 puede escribirse como

$$
\frac{d^{2} q^{\mu}}{d s^{2}}+\Gamma_{\lambda \nu}^{\mu} \frac{d q^{\lambda}}{d s} \frac{d q^{\nu}}{d s}+\frac{d q^{\mu}}{d s} \frac{\Omega}{d s}-\partial^{\mu} \Omega=0
$$




\subsection{GEOMETRODINÁMICA Y GENERALIZACIÓN DELTEOREMA DE TRABAJOS VIR}

Si consideramos ahora solamente trayectorias que son geodésicas para la métrica de Jacobi $\mathcal{G}_{\mu \nu}$, los dos primeros términos del primer miembro de ecuación (6.19) se anulan, quedando simplificada como

$$
\frac{d q^{\mu}}{d s} \frac{d \Omega}{d s}-\partial^{\mu} \Omega=\frac{d q^{\mu}}{d s}\left(\partial_{\alpha} \Omega \frac{d q^{\alpha}}{d s}\right)-\partial^{\mu} \Omega=0
$$

Finalmente, cambiando el parámetro de longitud $s$ a tiempo físico $t$ utilizando la relación $d s=2(E-V(\mathbf{q})) d t=2 W d t \rightarrow d t / d s=1 /(2 W)$, ecuación 6.20 se deduce que

$$
\frac{d q^{\mu}}{d t}\left(\partial_{\alpha} \Omega \frac{d q^{\alpha}}{d t}\right)=4 W^{2} \partial^{\mu} \Omega
$$

Para 2D, la expresión ( refeq:27) se puede escribir como

$$
\left\{\begin{array}{l}
\frac{d q^{1}}{d t}\left(\partial_{1} \Omega \frac{d q^{1}}{d t}+\partial_{2} \Omega \frac{d q^{2}}{d t}\right)=4 W^{2} \mathcal{G}^{11} \partial_{1} \Omega \\
\frac{d q^{2}}{d t}\left(\partial_{1} \Omega \frac{d q^{1}}{d t}+\partial_{2} \Omega \frac{d q^{2}}{d t}\right)=4 W^{2} \mathcal{G}^{22} \partial_{2} \Omega
\end{array}\right.
$$

La solución de estas ecuaciones viene dada implícitamente por $\mathcal{G}^{22} \partial_{2} \Omega\left(d q^{1} / d t\right)-$ $\mathcal{G}^{11} \partial_{1} \Omega\left(d q^{2} / d t\right)=0$ o, equivalentemente,

$$
a^{22} \partial_{2} \Omega \frac{d q^{1}}{d t}-a^{11} \partial_{1} \Omega \frac{d q^{2}}{d t}=0
$$

dado que la métrica de Jacobi $\mathcal{G}_{\mu \nu}(\mathbf{q})$ es una transformación conforme. En el caso de que $a_{i j}(\mathbf{x})=\delta_{i j}$, la expresión ( refeq:29) puede ser interpretada geométricamente como

$$
\left\langle\nabla \Omega, \gamma^{\perp}\right\rangle=0 \Rightarrow \nabla \Omega \| \gamma
$$

donde $\langle\cdot, \cdot\rangle$ el la métrica inicial en $\mathbb{R}^{2}$, es decir, $a_{\mu \nu}(\mathbf{q})$ en coordenadas, $\mathrm{y}$ $\gamma=\frac{d q^{1}}{d t} \mathbf{e}_{1}+\frac{d q^{2}}{d t} \mathbf{e}_{2}$. La misma condición de estabilidad se mantiene para el caso $\Omega(\mathbf{q})=\ln V(\mathbf{q})$ considerado anteriormente.

Las ecuaciones para un sistema 3D son

$$
\left\{\begin{array}{l}
\frac{d q^{1}}{d t}\left(\partial_{1} \Omega \frac{d q^{1}}{d t}+\partial_{2} \Omega \frac{d q^{2}}{d t}+\partial_{3} \Omega \frac{d q^{3}}{d t}\right)=4 W^{2} \mathcal{G}^{11} \partial_{1} \Omega \\
\frac{d q^{2}}{d t}\left(\partial_{1} \Omega \frac{d q^{1}}{d t}+\partial_{2} \Omega \frac{d q^{2}}{d t}+\partial_{3} \Omega \frac{d q^{3}}{d t}\right)=4 W^{2} \mathcal{G}^{22} \partial_{2} \Omega \\
\frac{d q^{3}}{d t}\left(\partial_{1} \Omega \frac{d q^{1}}{d t}+\partial_{2} \Omega \frac{d q^{2}}{d t}+\partial_{3} \Omega \frac{d q^{3}}{d t}\right)=4 W^{2} \mathcal{G}^{33} \partial_{3} \Omega
\end{array}\right.
$$


Su solución cumplirá

$$
\left\{\begin{array}{l}
\mathcal{G}^{11} \partial_{1} \Omega \frac{d q^{2}}{d t}-\mathcal{G}^{22} \partial_{2} \Omega \frac{d q^{1}}{d t}=0 \\
\mathcal{G}^{22} \partial_{2} \Omega \frac{d q^{3}}{d t}-\mathcal{G}^{33} \partial_{3} \Omega \frac{d q^{2}}{d t}=0 \\
\mathcal{G}^{11} \partial_{1} \Omega \frac{d q^{3}}{d t}-\mathcal{G}^{33} \partial_{3} \Omega \frac{d q^{1}}{d t}=0
\end{array}\right.
$$

o alternativamente las mismas expresiones cambiando $\mathcal{G}^{i j}$ por $a^{i j}$. Estas soluciones también puedes ser expresadas de una manera más compacta como

$$
\left|\begin{array}{ccc}
\mathbf{e}_{1} & \mathbf{e}_{2} & \mathbf{e}_{3} \\
a^{11} \partial_{1} \Omega & a^{22} \partial_{2} \Omega & a^{33} \partial_{3} \Omega \\
\frac{d q^{1}}{d t} & \frac{d q^{2}}{d t} & \frac{d q^{3}}{d t}
\end{array}\right|=0 .
$$

De nuevo, para $a_{i j}=\delta_{i j} \mathrm{y} / \mathrm{o} \Omega(\mathbf{q})=\ln V(\mathbf{q})$ obtenemos una interpretación geométrica de esta solución análoga a ecuación $(6.23)$, excepto que ahora el producto vectorial en $\mathbb{R}^{3}$ debe sustituir al producto escalar en $\mathbb{R}^{2}$ i.e. $\left|\nabla \Omega \wedge \gamma^{\perp}\right|=0$. Las soluciones no dependen de la métrica conforme, $\mathcal{G}_{i j}(\mathbf{q})$, pero sí de la métrica inicial, $a_{i j}(\mathbf{q})$.

Llamaremos a las ecuaciones (6.23) y 6.27) condición catenaria para trayectorias tanto en $2 \mathrm{D}$ como en $3 \mathrm{D}$.

\subsection{Aplicación al sistema Hénon-Heiles 2D}

En 1964 Hénon y Heiles [Weinan et al., 2007] definieron el siguiente sistema dinámico hamiltoniano para el estudio del movimiento de una estrella alrededor del centro de una galaxia, asumiendo que el movimiento podía restringirse al plano $(x, y)$

$$
\begin{aligned}
& H\left(x, y, P_{x}, P_{y}\right)=\mathcal{T}+V \\
& =\frac{1}{2}\left(P_{x}^{2}+P_{y}^{2}\right)+\frac{1}{2}\left(x^{2}+y^{2}\right)+x^{2} y-\frac{1}{3} y^{3} .
\end{aligned}
$$

La función potencial tiene simetría $C_{3 v}$ (triangular) y está acotada para energías hasta $E \leq 1 / 6$. Más allá de esta energía existen tres canales de escape, apuntando a los angulos polares $\pi / 2,7 \pi / 6$, and $11 \pi / 6$, atravesando los tres puntos de silla existentes de la función potencial localizados en $(x, y)=(0,1),( \pm \sqrt{3} / 2,-1 / 2)$.

Analizaremos la dinámica integrando las trayectorias numéricamente con condiciones iniciales elegidas aleatoriamente dentro del espacio de fases para energías 

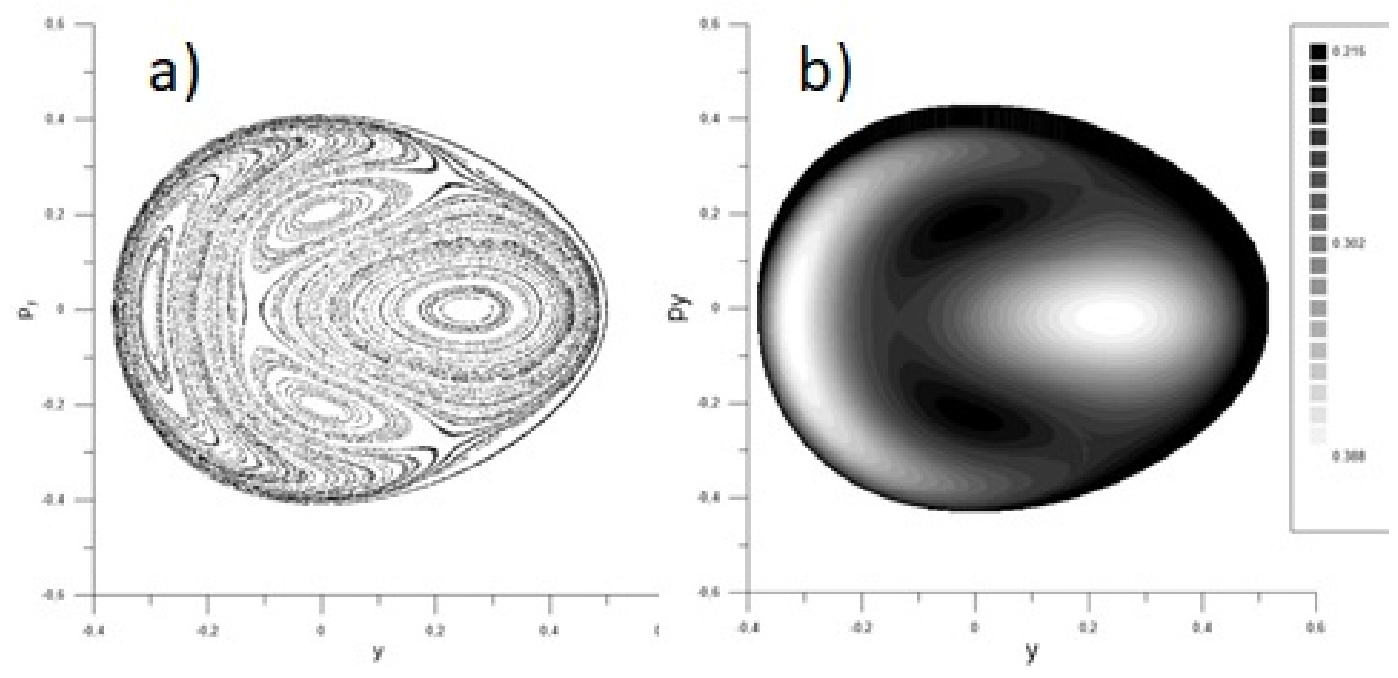

Figura 6.2: (a) PSS compuesta para $x=0$ and $P_{x}<0$, y (b) $I=\int_{\gamma} V(x, y) d s$ para el sistema Hénon-Heiles a $E=1 / 12$.

$E=1 / 12,1 / 8$, y $1 / 6$, extrayendo información dinámica calculando también las PSS ya definidas utilizando el plano de sección definido por $x=0$ and $P_{x}<0$. Esto nos permitirá distinguir las regiones del espacio de fases con movientos regulares de aquellas con movimientos caóticos. También las órbitas periódicas dejarán su huella en la PSS, pues marca un número entero de puntos fijos (que se corresponde con el orden de la resonancia) con islas alrededor de ellos y una banda estocástica entre ellas, como indica el conocido teorema de Poincaré-Birkhoff. Birkhoff, 1931.

\subsubsection{GVWP aplicado al sistema Hénon-Heiles 2D}

Primero, comprobaremos si la hipótesis lanzada en la sección previa 6.3.1 es correcta. Para ello, calcularemos los valores para la integral $I=\int_{\gamma} V(x, y) d s$ a lo largo de las mismas trayectorias de las que calculamos PSS, y comprobamos que las trayectorias más estables se corresponden a extremos de esta magnitud.

Obtenemos resultados para energía, $E=1 / 12$ como se muestra en Fig. 6.3. Como se ve en la PSS compuesta [panel (a)] todos los movimientos son regulares ya que aparecen como curvas cerradas, resultado de las intersecciones de los correspondientes toros invariante con el plano de sección. La presencia de con puntos fijos elípticos importantes localizados en $\left(y, P_{y}\right)=(0,0,2040)$, $(0,2545,0)$, respectivamente, con las correspondientes estructuras de islas al- 
rededor se ven claramente.

También, la posición de dos puntos fijos hiperbólicos se adivinan en $(-0,1200,0,0045),(0,205,-0,283)$, respectivamente, aunque la correspondiente banda estocástica es demasiado estrecha puede ser apreciada. Este resultado es la consecuencia de un valor muy bajo de la energía total E. Por otro lado, como se indica en la Fig. 6.2(b) la integral de acción $I$ muestra que las trayectorias más estables se corresponden con extremos, tanto mínimos como máximos de $I$.

Los valores de la integral $I$ a lo largo de trayectorias, aunque correcto atendiendo al carácter estacionario de las soluciones del problema variacional como se muestra en Fig. 6.2(b), no descrimina correctamente entre trayectorias estables e irregulares.

Con el fin de conseguir un indicador adecuado, proponemos discutir los resultados para las fluctuaciones de $I$ como hacemos en la siguiente sección.

\subsection{Definiendo el indicador de caos}

Asumimos que un indicador de caos debe depender de la dinámica del sistema y, por tanto, de la historia de la trayectoria. En esta línea, proponemos definir un observable que dependa de $I$ a lo largo de la trayectoria que determine el nivel de estabilidad de la misma. En nuestro caso, usaremos un observable derivado de la aplicación del VVWP, como indicamos previamente.

Estudiamos la fluctuación del escalar $\left\langle\nabla \Omega, \gamma^{\perp}\right\rangle$ para sistemas 2D, o $\left|\nabla \Omega \wedge \gamma^{\perp}\right|$ en el caso 3D con tensor métrico $a_{i j}(\mathbf{q})=\delta_{i j}$, y $\Omega(\mathbf{q})=\ln V(\mathbf{q})$. Para un sistema dinámico en general en dos o tres dimensiones con tensor de masa $a_{i j}(\mathbf{q})$ el escalar definido previamente se define igual pero sin la interpretación geométrica derivada del tensor unidad. Siguiendo la expresión Eq. (6.23), definimos el observable como

$$
\mathcal{O}(\mathbf{q}(t))=\frac{1}{|\nabla V||\dot{\gamma}(t)|}\left(a^{22} \partial_{2} V \frac{d q^{1}}{d t}-a^{11} \partial_{1} V \frac{d q^{2}}{d t}\right)
$$

y definimos el indicador de caos $\sigma_{\gamma}$ como la fluctuación de $\mathcal{O}$ a lo largo de la trayectoria $\gamma$ como

$$
\begin{gathered}
\sigma_{\gamma}(s)=\frac{\sqrt{\left\langle\left(\mathcal{O}-\langle\mathcal{O}\rangle_{t}\right)^{2}\right\rangle}}{t}, \text { con } \\
\langle\mathcal{O}\rangle_{\tau}=\frac{1}{\tau} \int_{0}^{\tau} \mathcal{O}(\xi) d \xi \\
\left\langle\left(\mathcal{O}-\langle\mathcal{O}\rangle_{t}\right)^{2}\right\rangle=\int_{0}^{t}\left(\mathcal{O}(\tau)-\langle\mathcal{O}\rangle_{\tau}\right)^{2} d \tau
\end{gathered}
$$



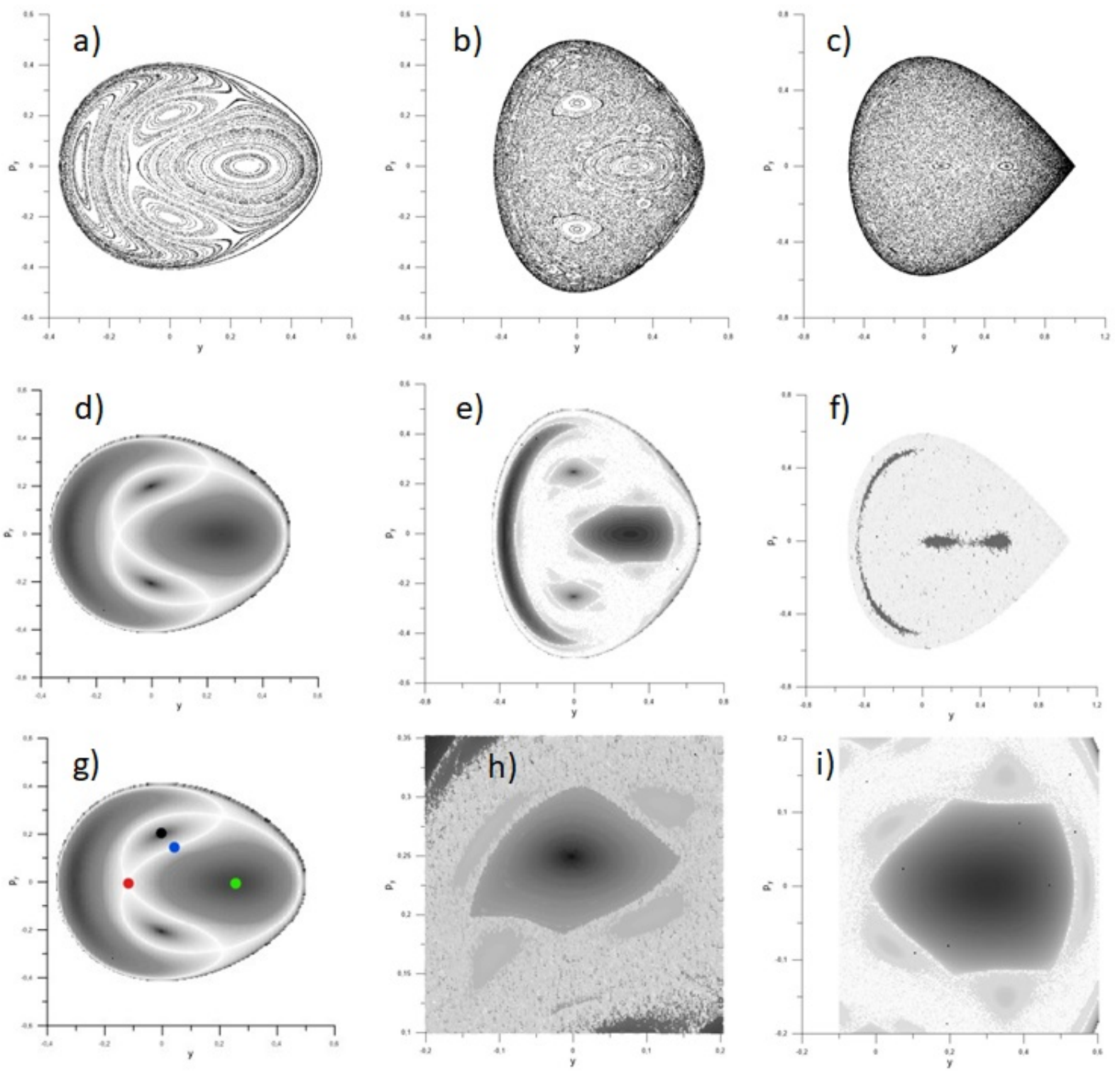

Figura 6.3: (a)-(c) Superficie de sección de Poincaré compuestas $x=0$ and $P_{x}<0, y$ (d)-(f) el indicador de caos $\sigma_{\gamma}$ definido en ecuación (6.30 y calculado para una longitud geodésica total de $s=100$ para el sistema de Henon-Heiles a $E=1 / 12$ (a) y (d), 1/8 (b) y (e), y $1 / 6$ (c) y (f). Las posiciones de cuatro trayectorias particulares para $E=1 / 12$, localizadas en $\left(y, P_{y}\right)=(0,0,2040)$ (negra), $(0,2545,0)$ (verde), $(-0,1200,0,0045)$ (roja), and $(0,1000,0,1850)$, que será analizada más tarde en Fig. 7.1. están indicadas con puntos coloreados. Expandimos $\sigma_{0}$ en dos zonas resonantes para $E=1 / 8$ son representadas en los paneles (g) y (h).

para un valor constante de la longitud de $\operatorname{arco} s=2 \int_{0}^{t}(E-V(\mathbf{q}(t))) d t=L$. En la ecuación 6.30$),\langle\ldots\rangle_{t}$ indica la media temporal en el intervalo $[0, t]$. Como se explica en la sección 6.3.1 cada trayectoria necesita un intervalo de tiempo físi- 
co diferente para alcanzar la longitud $L$. Como se indica en la ecuación (6.23) las trayectorias más estable se corresponden a valores nulos del observable $\mathcal{O}$ tal que analizando esta fluctuación podemos inferir el nivel de estabilidad para la trayectoria $\gamma$.

\subsubsection{Análisis de trayectorias}

Analizaremos cómo funciona nuestro indicador de caos cuando se aplica a trayectorias específicas. Para ello elegimos cuatro de ellas para energía $E=$ 1/12 correspondiéndose con los puntos coloreados indicados en la Fig. 6.3(g). Dos de ellas son órbitas periódicas estables, una tercera se corresponde con un punto fijo hiperbólico y la cuarta está situada en la correspondiente separatriz, donde el movimiento es caótico. Estas órbitas en el espacio figurativo $(x, y)$ se muestran en la Fig. 6.4 paneles (a)-(d), y la evolución temporal asociada de nuestro indicador de caos en el panel (e). Realizamos varios comentarios. En primer lugar, nuestro indicador de caos distingue correctamente las trayectorias estables/regulares de las inestables/caóticas. Observamos como el valor de $\sigma_{\gamma}$ es menor en el primer caso y mayor en el segundo. Segundo, asigna el mismo valor de indicador a ambas trayectorias irregulares, porque están en la misma banda estocástica.

Tercero, nuestro indicador asigna diferentes valores a las dos trayectorias estables consideradas, asignando diferentes grados de estabilidad a regiones regulares.

\subsection{Resultados numéricos}

Presentamos en la Fig. 6.3 nuestros resultados para la PSS y el indicador de caos $\sigma_{\gamma}$ definido en la ecuación (6.30) para diferentes valores de la energía, hemos tomado $E=1 / 12,1 / 8$, and $1 / 6 . \sigma_{\gamma}$ se representa utilizando diferentes escalas de grises como antes. Aquí colores más claros indican valores menores de $\sigma_{\gamma}$ y los más oscuros lo contrario, es decir, colores más oscuros indican la posición de trayectorias más regulares mientras que los más claros indican irregularidad y caos. Comenzando con el menor nivel de energía $E=1 / 12$, vemos el panel (d) que tenemos estas dos clases de colores, indicando la posición de las islas elípticas y bandas estocásticas respectivamente, coincidente con los resultados de las PSS en el panel (a), comentado anteriormente en relación a la Fig. 6.3 .

Otro interesante punto es que $\sigma_{\gamma}$ muestra un color más oscuro en los dos puntos fijos elípticos localizados en $y=0$ que para los otros dos localizados 

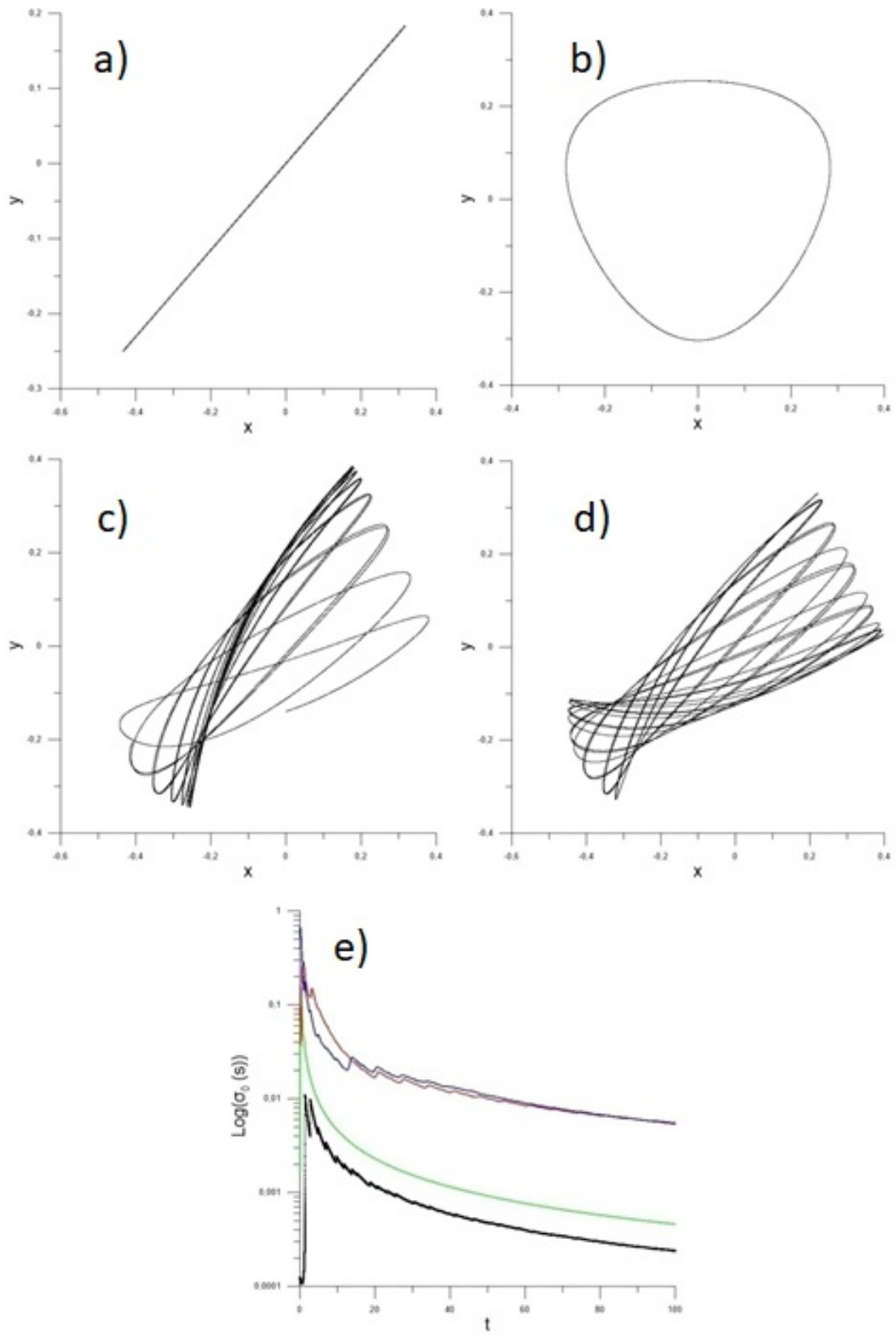

Figura 6.4: Trayectorias regulares (a)(verde),(b)(negro) y caóticas (c),(d) correspondiendo a las condiciones iniciales indicadas en Fig. 6.4(g). (e) Evolución correspondiente al indicador de caos $\sigma_{\gamma}$ utilizando el mismo código de colores.

en la línea $p_{y}=0$, indicando que nuestro indicador de caos puede detectar diferentes grados de estabilidad entre ellos.

Los resultados para el nivel de energía mayor $E=1 / 8$ se muestra en los 
paneles (b) y (e). En este caso, la PSS (ver dibujo en el panel (b)) muestra que tenemos esencialmente la misma estructura global que para el nivel de energía $E=1 / 12$, con los mismos puntos fijos tanto elípticos como hiperbólicos, aunque la figura ha sufrido cambios importantes. En particular, y como dice el teorema de Kolmogorov-Arnold-Moser Arnold, 1963, un gran número de toros invariantes han sido destruidos, lo que tiene como resultado un importante ensanchamiento de la región caótica a lo largo de la variedad que emana de los puntos hiperbólicos.

También, las islas alrededor de la estructura de islas se muestran con mayor claridad, y una resonancia importante 1:5 se hace visible alrededor del punto fijo en $\left(y=0, P_{y}=0,204\right)$. Nuestro indicador de caos $\sigma_{\gamma}$ muestra resultados similares, de nuevo mostrando a este nivel de energía la diferencia en estabilidad encontrada previamente a $E=1 / 12$. Este efecto es estudiado más profundamente en los paneles (h) y (i), el primero muestra la región alrededor de puntos fijos elípticos menos estables localizados en la linea $y=0$, y el último muestra otra más estable sobre $P_{y}=0$. Observamos que esto también explica, fácilmente, porqué la región de estabilidad alrededor del primer punto elíptico desaparece antes a medida que $E$ crece, que el segundo. Finalmente Fig. 6.3(c) y (f) muestran los resultados correspondientes al mayor nivel de energía considerado $E=1 / 6$. En este nivel de energía la mayoría de los toros invariantes se rompen, y la PSS está dominada por el caos, aunque algunas islas pequeñas de regularidad son visibles, especialmente dos localizadas sobre el eje $P_{y}=0$, con color más oscuro de nuestro indicador de caos. 


\section{Capítulo 7}

\section{Simetría e integrabilidad}

Mostraremos que cuando la condición catenaria se calcula a lo largo de una trayectoria, minimizando el indicador de caos previamente definido en la expresión (6.30), se puede obtener una cantidad conservada lineal en los momentos conjugados $\left(p_{i}\right)$ alrededor de dicha trayectoria.

El hecho anterior puede relacionarse directamente con la proposición de David Gregory en relación a arcos de la Mecánica Estructural que desde 1697 afirma: "Un arco de cualquier forma solamente puede estar en equilibrio si podemos dibujar una catenaria contenida completamente dentro del mismo considerando el caso de un contacto sin friccón entre las dovelas" como se ilustra en la Fig. (7.1).

Nosotros abstraemos esta idea reinterpretando 'Un arco de cualquier forma'

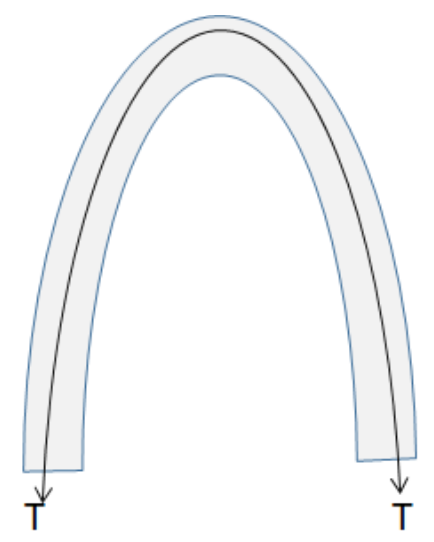

Figura 7.1: Curva catenaria incluida en un arco cualquiera 
como el conjunto completo de trayectorias próximas a la catenaria,es decir, con condiciones iniciales en regiones próximas a la catenaria. Podrían interpretarse las trayectorias como líneas tensionales. En la expresión para la trayectoria catenaria 6.23 o 6.27) podemos identificar la condición necesaria para la existencia de un campo vectorial Killing restringido a esta trayectoria como veremos más adelante, de tal modo que una cantidad conservada podría ser definida en una región próxima a la trayectoria con la consecuente estabilidad local.

\subsection{Simetría: vectores y campos Killing (KVF)}

Si $\gamma(s)$ es una curva geodésica para la métrica $g_{\mu \nu}$ y si existe un campo vectorial $X$ que cumple

$$
\frac{d}{d s}\left(X_{i} v^{i}\right)=\frac{1}{2} v^{j} v^{i}\left(\nabla_{i} X_{j}+\nabla_{j} X_{i}\right)=\nabla_{(i} X_{j)}=0
$$

con $\nabla_{i} X_{j}=\frac{\partial X_{j}}{\partial x^{i}}-\Gamma_{j i}^{k} X_{k}$ y $v^{i}=\frac{d q^{i}}{d s}$ el vector tangente a la geodésica, entonces el campo vectorial $X$ se denomina campo vectorial Killing, KVF, (Killing Vectorial Field) y la conservación de $X_{i} v^{i}$ es equivalente a $\nabla_{(i} X_{j)}=0$.

Considerando la métrica de Jacobi $g_{i j}=2(E-V(\mathbf{q})) a_{i j}$, entonces

$$
J(q, p)=X_{i}(q) \frac{d q^{i}}{d s}=\frac{1}{2(E-V(q))} X_{i}(q) a^{i l} p_{l}
$$

es una constante de movimiento a lo largo de la geodésica.

Las transformaciones de simetría para variedades Riemannianas son simetrías locales, esto es, aquellas $x^{\prime i}=x^{\prime i}\left(x^{m}\right)$ que mantienen el tensor métrico forminvariante. Es decir.se cumple

$$
g_{i j}^{\prime}(x)=g_{i j}(x)
$$

cuando se consideran transformaciones de coordenadas infinitesimales del tipo

$$
x^{\prime i}=x^{i}+\xi^{i}(x),
$$

con $\xi^{i}(x)$ campo vectorial infinitesimal $\delta_{L} g_{i j}=g_{i j}^{\prime}(x)-g_{i j}(x)=0$, donde $\delta_{L}$ se denomina derivada de Lie.

Si la derivada de Lie se anula, entonces $\delta_{L} g_{i j}=-\nabla_{i} \xi_{k}-\nabla_{k} \xi_{i}=0$, que se corresponde con la expresión anterior (7,1), es decir, $\nabla_{i} X_{j}+\nabla_{j} X_{i}=0$

Los campos KVF determinan las isometrías locales.

$$
\begin{aligned}
& X_{i} \frac{d q^{i}}{d s} \text { conservada } \Longleftrightarrow \\
& \nabla_{i} X_{j}+\nabla_{j} X_{i}=0 \Longleftrightarrow \\
& \delta_{L} g_{i j}=0 \text { isometría local } \equiv \text { simetría }
\end{aligned}
$$




$$
\nabla_{i} X_{j}+\nabla_{j} X_{i}=0
$$

y equivalen a la existencia de, al menos, una cantidad conservada $J$ lineal en los momentos conjugados $p_{i}$ tal que

$$
\{H(q, p), J(q, p)\}=\frac{\partial H}{\partial q^{i}} \frac{\partial J}{\partial p_{i}}-\frac{\partial H}{\partial p_{i}} \frac{\partial J}{\partial q^{i}}=0
$$

y

$$
J(q, p)=\sum_{i} C_{i}(q) p_{i}
$$

se conserva tal que podemos asociar un campo vectorial de componentes

$$
X_{k}=[E-V(q)] C_{k}(q)
$$

En coordenadas, el sistema de ecuaciones para $\nabla_{i} X_{j}+\nabla_{j} X_{i}=0$ se puede expresar como

$\underline{\text { Si } a_{i j}(q)=\delta_{i j}}$

$$
\begin{gathered}
{[E-V(q)]\left[\frac{\partial C_{i}(q)}{\partial q^{j}}+\frac{\partial C_{j}(q)}{\partial q^{i}}\right]-\delta_{i j} \sum_{k=1}^{N} \frac{\partial V}{\partial q^{k}} C_{k}(q)=0,} \\
E-V(q)=\frac{1}{2} \frac{d q^{k}}{d t} \frac{d q^{i}}{d t} \delta_{i k}=\frac{1}{2} \frac{d q^{k}}{d t} p_{k}=\frac{1}{2} p_{k} p_{l} \delta^{k l}=\frac{1}{2} \sum_{k=1}^{N} p_{k}^{2}, \\
\frac{1}{2} \sum_{k=1}^{N} p_{k}^{2}\left[\frac{\partial C_{i}(q)}{\partial q^{j}}+\frac{\partial C_{j}(q)}{\partial q^{i}}\right]-\delta_{i j} \sum_{k=1}^{N} \frac{\partial V}{\partial q^{k}} C_{k}(q)=0,
\end{gathered}
$$

generando el sistema de ecuaciones

$$
\begin{array}{cc}
\frac{\partial C_{i}(q)}{\partial q^{j}}+\frac{\partial C_{j}(q)}{\partial q^{i}}=0 & i \neq j, i, j=1, \cdots, N, \\
\frac{\partial C_{i}(q)}{\partial q^{i}}=0 & i=1, \cdots, N, \\
\sum_{k=1}^{N} \frac{\partial V}{\partial q^{k}} C_{k}(q)=0 &
\end{array}
$$

Si $a_{i j}(q)$ (general)

$$
\begin{gathered}
{[E-V(q)]\left[\frac{\partial C_{i}(q)}{\partial q^{j}}+\frac{\partial C_{j}(q)}{\partial q^{i}}\right]} \\
-a^{m l}\left\{\partial_{i} a_{j l}+\partial_{j} a_{l i}-\partial_{l} a_{i j}\right\} C_{m}(q)-\partial_{l} V a^{l m} a_{i j} C_{m}=0,
\end{gathered}
$$


donde $E-V(q)=\frac{1}{2} a_{k i} \frac{d q^{k}}{d t} \frac{d q^{i}}{d t}=\frac{1}{2} \frac{d q^{k}}{d t} p_{k}=\frac{1}{2} a^{k l} p_{k} p_{l}$, es decir

$$
\begin{gathered}
\frac{1}{2} a^{k l} p_{k} p_{l}\left[\frac{\partial C_{i}(q)}{\partial q^{j}}+\frac{\partial C_{j}(q)}{\partial q^{i}}\right] \\
-a^{m l}\left\{\partial_{i} a_{j l}+\partial_{j} a_{l i}-\partial_{l} a_{i j}\right\} C_{m}(q)-\partial_{l} V a^{l m} a_{i j} C_{m}=0 .
\end{gathered}
$$

Por tanto el sistema de ecuaciones es

$$
\left\{\begin{array}{l}
\frac{\partial C_{i}(q)}{\partial q^{j}}+\frac{\partial C_{j}(q)}{\partial q^{i}}-a^{m l}\left\{\partial_{i} a_{j l}+\right. \\
\left.\partial_{j} a_{l i}-\partial_{l} a_{i j}\right\} C_{m}(q)=0 \\
2\left[\frac{\partial C_{i}(q)}{\partial q^{i}}-a^{i i} \partial_{i} a_{i i} C_{i}(q)\right]+ \\
a^{m l} \partial_{l} a_{i i} C_{m}(q)=0 \\
\frac{\partial V}{\partial q^{l}} a^{l m} C_{m}(q)=0 .
\end{array} \quad i=1, \cdots, N,\right.
$$

La última ecuación en (7.14) se denomina ecuación de compatibilidad.

La integrabilidad es equivalente a encontrar un número de simetrías igual a la dimensión de la variedad mecánica.

\subsubsection{Problema de Kepler 2D en coordenadas polares}

En este caso, la métrica de Jacobi viene dada por

$$
g_{i j}=2(E-V(r)) a_{i j}(r, \theta)=2(E-V(r))\left(\begin{array}{cc}
1 & 0 \\
0 & r^{2}
\end{array}\right)
$$

y el campo KVF adopta la forma

$$
\begin{gathered}
\bar{X}(r, \theta)=X_{r}(r, \theta) \frac{\partial}{\partial r}+X_{\theta}(r, \theta) \frac{\partial}{\partial \theta} \\
\bar{X}(r, \theta)=[E-V(r)] C_{r}(r, \theta) \frac{\partial}{\partial r}+[E-V(r)] C_{\theta}(r, \theta) \frac{\partial}{\partial \theta} .
\end{gathered}
$$

Como $a_{i j} \neq \delta_{i j}$ entonces resolvemos el sistema de ecuaciones 7,14 con

índice $1 \longleftrightarrow$ variable $r$

índice $2 \longleftrightarrow$ variable $\theta$

$i=1, j=2$

$$
\frac{\partial C_{\theta}}{\partial r}+\frac{\partial C_{r}}{\partial \theta}-\frac{2}{r} C_{\theta}=0
$$

$i=j=1$

$$
\frac{\partial C_{r}}{\partial r}=0
$$


$i=j=2$

$$
\frac{\partial C_{\theta}}{\partial \theta}+r C_{r}=0
$$

Ecuación de compatibilidad

$$
\begin{gathered}
\partial_{r} V C_{r}=0, \\
\text { if } \frac{\partial V}{\partial r} \neq 0 \quad \Rightarrow C_{r}=0 .
\end{gathered}
$$

(Por ser central será independiente de la forma funcional de $V(r)$ ).

La solución para este sistema de ecuaciones será

$$
C_{r}(r, \theta)=0 \text { and } C_{\theta}(r, \theta)=k_{0} r^{2}, \quad k_{0}=\text { constante }
$$

por lo que el correspondiente $K V F$ viene dado por

$$
\bar{X}(r, \theta)=\underbrace{[E-V(r)] C_{r}(r, \theta)}_{X_{r}} \frac{\partial}{\partial r}+\underbrace{[E-V(r)] C_{\theta}(r, \theta)}_{X_{\theta}} \frac{\partial}{\partial \theta}=\underbrace{[E-V(r)] k_{0} r^{2}}_{X_{\theta}} \frac{\partial}{\partial \theta}
$$

y la constante de movimiento por

$$
\begin{gathered}
J\left(r, \theta, \frac{d r}{d t}, \frac{d \theta}{d t}\right)=X_{\theta}(r, \theta) \frac{d \theta}{d s}=\underbrace{[E-V(r)] k_{0} r^{2}}_{X_{\theta}} \underbrace{\frac{d \theta}{d s}}_{\frac{d \theta}{d t} \frac{d t}{d s}}= \\
{[E-V(r)] k_{0} r^{2} \frac{d \theta}{d t} \frac{1}{2[E-V(r)]} .}
\end{gathered}
$$

Es decir,

$$
J\left(r, \theta, \frac{d r}{d t}, \frac{d \theta}{d t}\right)=K_{0} \frac{r^{2}}{2} \frac{d \theta}{d t} \quad,
$$

donde $\frac{r^{2}}{2} \frac{d \theta}{d t}$ se llama velocidad areolar. Por tanto

$$
J\left(r, \theta, p_{r}, p_{\theta}\right)=\frac{K_{0}}{2} p_{\theta}
$$

\subsubsection{Problema de Kepler 2D en coordenadas cartesia- nas}

La función potencial viend definida como

$$
V(x, y)=\frac{-G M}{\sqrt{x^{2}+y^{2}}}
$$


La métrica Jacobi tendrá la forma

$$
g_{i j}=2(E-V(x, y)) \delta_{i j}=2(E-V(x, y))\left(\begin{array}{ll}
1 & 0 \\
0 & 1
\end{array}\right)
$$

y el KVF adopta la forma

$$
\bar{X}(x, y)=X_{x}(x, y) \frac{\partial}{\partial x}+X_{y}(x, y) \frac{\partial}{\partial y},
$$

es decir

$$
\bar{X}(x, y)=[E-V(x, y)] C_{x}(x, y) \frac{\partial}{\partial x}+[E-V(x, y)] C_{y}(x, y) \frac{\partial}{\partial y} .
$$

Como $a_{i j}=\delta_{i j}$ entonces resolvemos el sistema de ecuaciones 7.14 con

índice $1 \longleftrightarrow$ variable $x$

índice $2 \longleftrightarrow$ variable $y$

$i=1, j=2$

$$
\frac{\partial C_{y}}{\partial x}+\frac{\partial C_{x}}{\partial y}=0
$$

$i=j=1$

$$
\frac{\partial C_{x}}{\partial x}=0
$$

$i=j=2$

$$
\frac{\partial C_{y}}{\partial y}=0
$$

Ecuación de compatibilidad

$$
\frac{\partial}{\partial x} V(x, y) C_{x}+\frac{\partial}{\partial y} V(x, y) C_{y}=0
$$

La solución para este sistema de ecuaciones será

$$
C_{x}(x, y)=\alpha \text { and } C_{y}(x, y)=-\alpha x \quad \alpha=\text { constante }
$$

el correspondiente $K V F$

$$
\begin{gathered}
\bar{X}(x, y)=\underbrace{[E-V(x, y)] C_{x}}_{X_{x}} \frac{\partial}{\partial x}+\underbrace{[E-V(x, y)] C_{y}}_{X_{y}} \frac{\partial}{\partial y}= \\
\underbrace{[E-V(x, y)] \alpha y}_{X_{x}} \frac{\partial}{\partial x}-\underbrace{[E-V(x, y)] \alpha x}_{X_{y}} \frac{\partial}{\partial y}
\end{gathered}
$$


y la constante de movimiento

$$
J\left(x, y, p_{x}, p_{y}\right)=X_{k} \frac{d q^{k}}{d s}=\frac{\alpha}{2}\left[y p_{x}-x p_{y}\right],
$$

donde

$$
J\left(x, y, p_{x}, p_{y}\right)=\frac{\alpha}{2}\left[y p_{x}-x p_{y}\right]=\bar{L}=\bar{r} \times \bar{p}
$$

es el momento angular.

Ahora necesitamos definir otro tipo de simetrías llamadas simetrías ocultas que no son tan fácilmente reconocibles y que irán asociadas a ciertos campos tensoriales.

\subsection{Campos tensoriales Killing}

Generalizando la expresión para cantidades conservadas, en lugar de $J=$ $X_{i} v^{i}$ la nueva constante de movimiento es

$$
J=K_{j_{1} j_{2} \cdots j_{r}} v^{j_{1}} v^{j_{2}} \cdots v^{j_{r}}
$$

con la condición

$$
\frac{d}{d s}\left(K_{j_{1} j_{2} \cdots j_{r}} v^{j_{1}} v^{j_{2}} \cdots v^{j_{r}}\right)=v^{j} \nabla_{j}\left(K_{j_{1} j_{2} \cdots j_{r}} v^{j_{1}} v^{j_{2}} \cdots v^{j_{r}}\right)=0 .
$$

El tensor $K_{j_{1} j_{2}} \cdots j_{r}$ se denomina campo tensorial Killing, (KTF) y considerando (7.1)

$$
\left.\frac{d}{d s}\left(K_{j_{1} j_{2} \cdots j_{r}} v^{j_{1}} v^{j_{2}} \cdots v^{j_{r}}\right)=v^{j_{1}} v^{j_{2}} \cdots v^{j_{r}} v^{j} \nabla_{(j} K_{j_{1} j_{2} \cdots j_{r}}\right),
$$

donde

$$
\nabla_{(j} K_{\left.j_{1} j_{2} \cdots j_{r}\right)}=\nabla_{j} K_{j_{1} j_{2} \cdots j_{r}}+\nabla_{j_{1}} K_{j j_{2} \cdots j_{r}}+\cdots \nabla_{j_{r}} K_{j_{1} j_{2} \cdots j_{r-1} j}
$$

Por tanto el campo tensorial debe cumplir

$$
\nabla_{(j} K_{\left.j_{1} j_{2} \cdots j_{r}\right)}=0
$$

Entonces el escalar

$$
J(q, p) \equiv K_{j_{1} j_{2} \cdots j_{r}} \frac{d q^{j_{1}}}{d s} \frac{d q^{j_{2}}}{d s} \cdots \frac{d q^{j_{r}}}{d s}
$$


es una constante de movimiento para el flujo geodésico en la variedad mecánica., obteniendo la equivalencia

$$
\{H, J\}=0 \Longleftrightarrow \nabla_{(j} K_{\left.j_{1} j_{2} \cdots j_{r}\right)}=0 .
$$

Una variedad riemanniana con coeficientes de conexión $\Gamma_{j k}^{i}$ que se derivan de la métrica cumple

$$
\begin{gathered}
\nabla_{i} g_{j k}=0 \\
\nabla_{(j} g_{\left.j_{1} j_{2}\right)}=\nabla_{j} g_{j_{1} j_{2}}+\nabla_{j_{1}} g_{j j_{2}}+\nabla_{j_{2}} g_{j_{1} j}=0
\end{gathered}
$$

Por tanto $g_{i j}$ es un $K T F \frac{d q^{i}}{d s}=\frac{d q^{i}}{d t} \frac{d t}{d s}=\frac{d q^{i}}{d t} \frac{1}{2[E-V(\mathbf{q})]}$.

Así la siguiente cantidad consevada $J$ es

$$
\begin{gathered}
J\left(q^{i}, \frac{d q^{i}}{d s}\right) \equiv g_{i j} \frac{d q^{i}}{d s} \frac{d q^{j}}{d s}= \\
2[(E-V(\mathbf{q}))] a_{i j} \frac{d q^{i}}{d s} \frac{d q^{j}}{d s}= \\
2[E-V(\mathbf{q})] a_{i j} \frac{d q^{i}}{d t} \frac{d q^{j}}{d t} \frac{1}{(2[E-V(\mathbf{q})])^{2}}= \\
\underbrace{a_{i j} \frac{d q^{i}}{d t} \frac{d q^{j}}{d t}}_{2 T=2(E-V(\mathbf{q}))} \frac{1}{2[E-V(\mathbf{q})]}=1
\end{gathered}
$$

\subsubsection{Buscando campos vectoriales Killing}

Si un sistema dinámico bidimensional contiene una trayectoria cumpliendo la condición catenaria con matriz de masa $a_{i j}=\delta_{i j}$ y $\Omega=\ln V$, la Eq. 6.23 toma la forma

$$
\frac{\partial V}{\partial q^{2}} \frac{d q^{1}}{d t}-\frac{\partial V}{\partial q^{1}} \frac{d q^{2}}{d t}=0
$$

Considerando las ecuaciones de Hamilton

$$
\begin{aligned}
& \frac{\partial H}{\partial q^{k}}=-\frac{d p_{k}}{d t} \\
& \frac{\partial H}{\partial p_{k}}=\frac{d q^{k}}{d t} \quad k=1,2,
\end{aligned}
$$

7.48 se transforma en

$$
\dot{p}_{1} p_{2}-\dot{p}_{2} p_{1}=0
$$

Así $p_{2} \neq 0$, es equivalente a

$$
\left(\frac{\dot{p_{1}}}{p_{2}}\right)=0 \Rightarrow \frac{p_{1}}{p_{2}}=K .
$$


con $K=$ constante. Entonces la ecuación (7.51) establece una cantidad consevada lineal en los momentos conjugados a lo largo de la trayectoria catenaria pero no necesariamente un $K V F$ definido sobre todo el espacio. La relación (7.48) implica la existencia de una cantidad conservada a lo largo de esta trayectoria y una región regular alrededor suyo.

Definiendo $C_{1}=\frac{\partial V}{\partial q^{2}}$ y $C_{2}=-\frac{\partial V}{\partial q^{1}}$, si cumplen 7.11 obtenemos una constante de movimiento como en la ecuación (7.7).

Estas ideas previas abstraen la proposición de David Gregory en Mecánica Estructural para la estabilidad de arcos, con la que empezabamos este capítulo: "Si cualquier arco de forma diferente al arco catenario mantiene su estabilidad, ello es debido a que una catenaria puede ser contenida completamente en su interior"

En relación a sistemas dinámicos la trayectoria catenaria está siempre incluida en una región regular como la curva catenaria lo está en el interior de los arcos estables arbitrarios.

La expresión (7.50) es válida para sistemas dinámicos bidimensionales con matriz de masa $a_{i j}=\delta_{i j}$, ahora mostraremos su aplicación en varios ejemplos.

\section{Oscilador armónico isótropo 2D}

EL hamiltniano del oscilador armónico isótropo 2D viene dado por

$$
H=\frac{1}{2}\left(p_{1}^{2}+p_{2}^{2}+\left(q^{1}\right)^{2}+\left(q^{2}\right)^{2}\right)
$$

En este caso la expresîA 'ón 7.48 toma la forma

$$
. q^{2} p_{1}-q^{1} p_{2}=0
$$

que es la expresion de un $K V F$. Obtenemos una cantidad conservada $J\left(q^{i}, p_{i}\right)$ como hemos definido en (7.7), tomando

$$
\begin{aligned}
& C_{1}\left(q^{i}\right)=q^{2} \\
& C_{2}\left(q^{i}\right)=-q^{1} .
\end{aligned}
$$

Se puede comprobar que $C_{i}\left(q^{i}\right)$ cumple Eq. (7.11) por lo que hemos encontrado a $K V F$ y una cantidad conservada

$$
J(q, p)=\sum_{i} C_{i}(q) p_{i}=q^{2} p_{1}-q^{1} p_{2}=\text { constant } .
$$

que, evidentemente cumple la condición necesaria para cantidades conservadas

$$
\{H(q, p), J(q, p)\}=\frac{\partial H}{\partial q^{i}} \frac{\partial J}{\partial p_{i}}-\frac{\partial H}{\partial p_{i}} \frac{\partial J}{\partial q^{i}}=0
$$




\section{Sistemas dinámicos con potencial central $2 \mathrm{D}$}

En este caso, el hamiltoniano se puede expresar como

$$
H=\frac{1}{2}\left(p_{1}^{2}+p_{2}^{2}\right)+V(r),
$$

con $r=\sqrt{\left(q^{1}\right)^{2}+\left(q^{2}\right)^{2}}$. ENtonces se tiene que

$$
\frac{\partial V}{\partial q^{i}}=\frac{\partial V}{\partial r} \frac{\partial r}{\partial q^{i}}=\frac{\partial V}{\partial r} \frac{q^{i}}{r} .
$$

Ssustituyendo en Eq. (7.48), se obtiene

$$
q^{2} p_{1}-q^{1} p_{2}=0 .
$$

Como en el ejemplo previo, la cantidad conservada $J\left(q^{i}, p_{i}\right)$ es

$$
J\left(q^{i}, p_{i}\right)=q^{2} p_{1}-q^{1} p_{2}=\text { constant } .
$$

$J$ es una función lineal en los momentos

$$
J(q, p)=\sum_{i} C_{i}(q) p_{i}
$$

con $C_{1}(q)=q^{2}$ y $C_{2}(q)=-q^{1}$. Con estas cantidads y resolviendo la ecuación (7.11) podemos asociar un $K V F$ de componentes

$$
\vec{X}=[E-V(q)] q^{2} \frac{\partial}{\partial q^{1}}-[E-V(q)] q^{1} \frac{\partial}{\partial q^{2}} .
$$

\section{Molécula de Toda 3D}

Consideremos el Hamiltoniano

$$
\begin{aligned}
& H=\frac{1}{2}\left(p_{1}^{2}+p_{2}^{2}+p_{3}^{2}\right)+V\left(q^{1}, q^{2}, q^{3}\right) \\
& V\left(q^{1}, q^{2}, q^{3}\right)=e^{q^{1}-q^{2}}+e^{q^{2}-q^{3}}-e^{q^{3}-q^{1}} .
\end{aligned}
$$

Expresamos la condición catenaria como 6.27), que en este caso toma la forma

$$
\left(\frac{\partial V}{\partial q^{3}}-\frac{\partial V}{\partial q^{2}}\right) p_{1}+\left(\frac{\partial V}{\partial q^{1}}-\frac{\partial V}{\partial q^{3}}\right) p_{2}+\left(\frac{\partial V}{\partial q^{2}}-\frac{\partial V}{\partial q^{1}}\right) p_{3}=0
$$

Para encontrar un $K V F$ a lo largo de una curva podemos identificar esta expresión con la ecuación de compatibilidad que aparece como última ecuación del sistema Eq. (7.11), es decir,

$$
\begin{aligned}
& C_{1}=\frac{\partial V}{\partial q^{3}}-\frac{\partial V}{\partial q^{2}}, \\
& C_{2}=\frac{\partial V}{\partial q^{1}}-\frac{\partial V}{\partial q^{3}}, \\
& C_{3}=\frac{\partial V}{\partial q^{2}}-\frac{\partial V}{\partial q^{1}} .
\end{aligned}
$$


En este caso

$$
\begin{aligned}
& C_{1}=e^{q^{1}-q^{2}}-2 e^{q^{2}-q^{3}}-e^{q^{3}-q^{1}}, \\
& C_{2}=e^{q^{1}-q^{2}}+e^{q^{2}-q^{3}}+2 e^{q^{3}-q^{1}}, \\
& C_{3}=-2 e^{q^{1}-q^{2}}+e^{q^{2}-q^{3}}-e^{q^{3}-q^{1}} .
\end{aligned}
$$

La ecuación de compatibilidad se cumple es decir,

$$
\begin{aligned}
& \frac{\partial V}{\partial q^{1}}\left(\frac{\partial V}{\partial q^{3}}-\frac{\partial V}{\partial q^{2}}\right)+ \\
& \frac{\partial V}{\partial q^{2}}\left(\frac{\partial V}{\partial q^{1}}-\frac{\partial V}{\partial q^{3}}\right)+ \\
& \frac{\partial V}{\partial q^{3}}\left(\frac{\partial V}{\partial q^{2}}-\frac{\partial V}{\partial q^{1}}\right)=0,
\end{aligned}
$$

pero para encontrar un $K V F$ necesitamos resolver todas las ecuaciones del sistema 7.11

$$
\begin{array}{ll}
\frac{\partial C_{1}}{\partial q^{1}}=e^{q^{3}-q^{1}}+e^{q^{1}-q^{2}} & =0, \\
\frac{\partial C_{2}}{\partial q^{2}}=e^{q^{2}-q^{3}}-e^{q^{1}-q^{2}} & =0, \\
\frac{\partial C_{3}}{\partial q^{3}}=e^{q^{3}-q^{1}}+e^{q^{2}-q^{3}} & =0, \\
\frac{\partial C_{1}}{\partial q^{2}}+\frac{\partial C_{2}}{\partial q^{1}}=2 \frac{\partial C_{3}}{\partial q^{3}} & =0, \\
\frac{\partial C_{1}}{\partial q^{3}}+\frac{\partial C_{3}}{\partial q^{1}}=2 \frac{\partial C_{2}}{\partial q^{2}} & =0, \\
\frac{\partial C_{2}}{\partial q^{3}}+\frac{\partial C_{3}}{\partial q^{2}}=2 \frac{\partial C_{1}}{\partial q^{1}} & =0 .
\end{array}
$$

Debido a la forma del potencial de Toda las tres últimas ecuaciones son redundantes y el sistema no tienen solución si las relaciones son como se han indicado en (7.65).

Por tanto, la única solución del sistema es la trivial

$$
\begin{aligned}
& C_{1}\left(q^{i}\right)=k_{1}, \\
& C_{2}\left(q^{i}\right)=k_{2}, \\
& C_{3}\left(q^{i}\right)=k_{3},
\end{aligned}
$$

con $k_{1}, k_{2}, k_{3}=$ const.

Debido a que la solución no tiene la forma indicada en 7.65 debemos comprobar de nuevo que se cumple la ecuación de compatibilidad es decir,

$$
\sum_{k=1}^{3} \frac{\partial V}{\partial q^{k}} C_{k}(q)=0 .
$$

Sustituyendo 7.68

$$
\frac{\partial V}{\partial q^{1}} k_{1}+\frac{\partial V}{\partial q^{2}} k_{2}+\frac{\partial V}{\partial q^{3}} k_{3}=0
$$

es decir

$$
\left\{\begin{array}{l}
\left.e^{q^{1}-q^{2}}+e^{q^{3}-q^{1}}\right) k_{1}+ \\
\left.e^{q^{2}-q^{3}}-e^{q^{1}-q^{2}}\right) k_{2}+ \\
\left.-e^{q^{2}-q^{3}}-e^{q^{3}-q^{1}}\right) k_{3}=0
\end{array}\right.
$$


equivalente a

$$
\begin{aligned}
& e^{q^{1}-q^{2}}\left(k_{1}-k_{2}\right)+ \\
& e^{q^{3}-q^{1}}\left(k_{1}-k_{3}\right)+ \\
& e^{q^{2}-q^{3}}\left(k_{2}-k_{3}\right)=0 .
\end{aligned}
$$

Una posible solución es $k_{1}=k_{2}=k_{3}=k=$ const.. Por tanto la cantidad conservada $J\left(q^{i}, p_{i}\right)$ como en (7.7) es

$$
J(q, p)=\sum_{i} C_{i}(q) p_{i}=k\left(p_{1}+p_{2}+p_{3}\right) .
$$

Comprobamos que cumple la condición de cantidad conservada

$$
\{J(q, p), H(q, p)\}=0
$$

Es importante observar cómo estudiando el $K V F$ a lo largo de trayectorias cumpliendo la condición catenaria hemos encontrado una cantidad conservada lineal en los momentos conjugados para todo el espacio de fases.

\subsection{Condiciones de integrabilidad para siste- mas 3D}

Para que un sistema dinámico Hamiltoniano sea integrable debemos encontrar tres constantes de movimiento en involución.

Obviamente la primera integral $I_{1}(\mathbf{q}, \mathbf{p})=H$ es la energía total. Por otro lado, muchas veces en la práctica no es difícil encontrar una segunda integral de movimiento pero la tercera es mucho más difícil de encontrar.

En esta sección describimos un método alternativo para detectar cuando algunos sistemas dinámicos conservativos son integrables. En lugar de buscar integrales primeras, buscaremos un tipo de subvariedades donde la dinámica sea absolutamente regular.

Si una trayectoria satiface la condición catenaria en 3D expresada como 7.63 se puede calcular a lo largo de esta trayectoria una integral primera lineal en los momentos conjugados. Esta primera integral viene dada por

$$
\begin{gathered}
I=\left(\frac{\partial V}{\partial q^{3}}-\frac{\partial V}{\partial q^{2}}\right) p_{1}+ \\
\left(\frac{\partial V}{\partial q^{1}}-\frac{\partial V}{\partial q^{3}}\right) p_{2}+\left(\frac{\partial V}{\partial q^{2}}-\frac{\partial V}{\partial q^{1}}\right) p_{3}=0
\end{gathered}
$$

apareciendo una region regular localizada alrededor de dicha trayectoria como se indicó en secciones previas. 
Nuestro objetivo es ver si se puede generalizar este resultado previo a todo el espacio $\mathbb{R}^{3}$ encontrando subvariedades bidimensionales generadas por trayectorias que cumplen la condición catenaria (7.63) y foliando densamente $\mathbb{R}^{3}$ hasta determinar la dinámica regular general y su integrabilidad.

Geométricamente, 7.75 implica que podría existir una subvariedad inmersa en $\mathbb{R}^{3}$ definida como $U(\mathbf{q})=k$, que llamaremos subvariedad catenaria, donde $\frac{d \mathbf{q}}{d t} \equiv \mathbf{p}=\left(p_{1}, p_{2}, p_{3}\right) \in T_{q} U(\mathbf{q})$ y $\nabla U(\mathbf{q})=\left(\frac{\partial V}{\partial q^{3}}-\frac{\partial V}{\partial q^{2}}, \frac{\partial V}{\partial q^{1}}-\frac{\partial V}{\partial q^{3}}, \frac{\partial V}{\partial q^{2}}-\frac{\partial V}{\partial q^{1}}\right)$ son ortogonales en $\mathbf{q}$.

Así mismo se cumple la igualdad

$$
\nabla V(\mathbf{q}) \cdot \nabla U(\mathbf{q}) \equiv 0
$$

donde $\cdot$ el producto escalar.

Cuando las trayectorias están incluidas en dicha subvariedad para diferentes valores de $k$, debido a la identidad (7.76) la dinámica para las trayectorias catenarias es laminar y el espacio $\mathbb{R}^{3}$ está foliado por subvariedades catenarias localmente regulares.

Para compronar analíticamente si estas subvariedades $U(\mathbf{q})=k$ existen para un hamiltoniano en particular tenemos que resolver la ecuación diferencial de primer orden 7.75 con $p_{i}=\frac{d q^{i}}{d t}$, que es equivalente a resolver la siguiente ecuación Pfaffiana en en espacio Euclideo tridimensinal

$$
\omega \equiv P(\mathbf{q}) d q^{1}+Q(\mathbf{q}) d q^{2}+R(\mathbf{q}) d q^{3}=0,
$$

donde $\mathbf{q} \in D \subset \mathbb{R}^{3}$ y $\omega$ una 1-forma diferencial con $\mathbf{q}=\left(q^{1}, q^{2}, q^{3}\right)$ y $P(\mathbf{q}), Q(\mathbf{q}), R(\mathbf{q}) \in C^{1}(D)$.

ElTeorema de Frobenius afirma que la condición necesaria y suficiente para que la ecuación Pfaffiana sea completamente integrables es

$$
d \omega \wedge \omega \equiv 0
$$

Esta condición para el caso tres dimensional es equivalente a

$$
P\left(\frac{\partial Q}{\partial q^{3}}-\frac{\partial R}{\partial q^{2}}\right)+Q\left(\frac{\partial R}{\partial q^{1}}-\frac{\partial P}{\partial q^{3}}\right)+R\left(\frac{\partial P}{\partial q^{2}}-\frac{\partial Q}{\partial q^{1}}\right)=0,
$$

que, en lenguaje vectorial se puede exresar como $(\mathbf{F}, \mathbf{F})=0$ with $\mathbf{F}=(P, Q, R)$.

En este caso podremos encontrar una función $\mu(\mathbf{q}) \neq 0$ como factor integrante tal que exista una función diferenciable $U(\mathbf{q})$ y

$$
d U \equiv \mu\left(P d q^{1}+Q d q^{2}+R d q^{3}\right)=0 .
$$


En coordenadas $\left(q^{i}\right)$ la ecuación 7.80 se corresponde a

$$
\frac{d U\left(q^{i}(t)\right)}{d t}=\mu\left(P \frac{d q^{1}}{d t}+Q \frac{d q^{2}}{d t}+R \frac{d q^{3}}{d t}\right)=0
$$

y para las condiciones iniciales $\mathbf{q}_{0}=\left(q_{0}^{1}, q_{0}^{2}, q_{0}^{3}\right)$ solamente existe un valor real $k$ tal que $U\left(\mathbf{q}_{0}\right)=k$.

Si esta igualdad se cumple la trayectoria con condiciones iniciales $\left(q_{0}^{i}\right) \in$ $U(\mathbf{q})=k$ y $\frac{d q^{i}}{d t} \in T_{\mathbf{q}} U(\mathbf{q})$ está siempre confinada en la variedad $U(\mathbf{q})=k$ tal que podemos reducir la dinámica tres dimensional a otra dos dimensional.

Esta afirmación restringe la caoticidad del sistema porque cualquier trayectoria con condiciones iniciales citadas anteriormente no puede escapar de esta subvariedad catenaria haciendo imposible que alcance cualquier región del espacio de fases.

Mostraremos que cuando se cumple la condición 7.79 todo $\mathbb{R}^{3}$ está foliado densamente por subvariedades catenarias $U(\mathbf{q})=k$ localmente regulares y por tanto toda la dinámica del sistema será regular.

\subsubsection{Condición de integrabilidad}

Sea un sistema mecánico con función hamiltoniana expresada en coordenadas canónicas $(\mathbf{q}, \mathbf{p})=\left(q^{1}, q^{2}, q^{3}, p_{1}, p_{2}, p_{3}\right)$ como

$$
H(\mathbf{q}, \mathbf{p})=\frac{1}{2} \sum_{i=1}^{3}\left(p_{i}\right)^{2}+V(\mathbf{q})
$$

y consideremos el campo vectorial $P(\mathbf{q}) \partial_{q^{1}}+Q(\mathbf{q}) \partial_{q^{2}}+R(\mathbf{q}) \partial_{q^{3}}$ en $\mathbb{R}^{3}$ con

$$
\begin{aligned}
& P(\mathbf{q})=\frac{\partial V}{\partial q^{3}}-\frac{\partial V}{\partial q^{2}} \\
& Q(\mathbf{q})=\frac{\partial V}{\partial q^{1}}-\frac{\partial V}{\partial q^{3}} \\
& R(\mathbf{q})=\frac{\partial V}{\partial q^{2}}-\frac{\partial V}{\partial q^{1}}
\end{aligned}
$$

Si la condición

$$
P\left(\frac{\partial Q}{\partial q^{3}}-\frac{\partial R}{\partial q^{2}}\right)+Q\left(\frac{\partial R}{\partial q^{1}}-\frac{\partial P}{\partial q^{3}}\right)+R\left(\frac{\partial P}{\partial q^{2}}-\frac{\partial Q}{\partial q^{1}}\right) \equiv 0
$$

se cumple identicamente, entonces existen subvariedades catenarias localmente regulares foliando densamente $\mathbb{R}^{3}$ de tal manera que las trayectorias catenarias están confinadas totalmente en hojas del tipo $U(\mathbf{q})=k$, induciendo que la dinámica completa sea regular y el sistema Hamiltoniano 7.82 integrable. 


\subsection{Aplicaciones}

Aplicaremos la condición anterior a diferentes sistemas dinámicos Hamiltonianos con tres grados de libertad.

\section{Molécula de Toda 3D}

El hamiltoniano está descrito po la expresión (7.62) donde la condición catenaria es

$$
P(\mathbf{x}) p_{1}+Q(\mathbf{x}) p_{2}+R(\mathbf{x}) p_{3}=0
$$

con

$$
\begin{aligned}
& P(\mathbf{x})=\frac{\partial V}{\partial q^{3}}-\frac{\partial V}{\partial q^{2}}=e^{q^{1}-q^{2}}-2 e^{q^{2}-q^{3}}-e^{q^{3}-q^{1}} \\
& Q(\mathbf{x})=\frac{\partial V}{\partial q^{1}}-\frac{\partial V}{\partial q^{3}}=e^{q^{1}-q^{2}}+e^{q^{2}-q^{3}}+2 e^{q^{3}-q^{1}} \\
& R(\mathbf{x})=\frac{\partial V}{\partial q^{2}}-\frac{\partial V}{\partial q^{1}}=-2 e^{q^{1}-q^{2}}+e^{q^{2}-q^{3}}-e^{q^{3}-q^{1}} .
\end{aligned}
$$

Se puede comprobar fácilmente que se cumple la condición de integrabilidad (7.79) con $F=(P(\mathbf{x}), Q(\mathbf{x}), R(\mathbf{x}))$, por lo que podemos concluir que el sistema hamiltoniano correspondiente a la molécula de Toda descrito por 7.62 es un sistema dinámico integrable.

Se observa que

$$
\frac{\partial V}{\partial q^{1}}+\frac{\partial V}{\partial q^{2}}+\frac{\partial V}{\partial q^{3}}=0
$$

de tal manera que, como se indicó en la sección previa, existe una primera integral inducida de la simetría de un KVF dado por

$$
\bar{I}=p_{1}+p_{2}+p_{3},
$$

encontrando dos constantes de movimiento

$$
\begin{aligned}
& H(\mathbf{q}, \mathbf{p}) \\
& \bar{I}(\mathbf{p})=p_{1}+p_{2}+p_{3}
\end{aligned}
$$

en involución, es decir,

$$
\{\bar{I}(\mathbf{p}), H(\mathbf{q}, \mathbf{p})\}=0
$$

\section{La red de Toda $3 \mathrm{D}$}

El hamiltoniano de la red de Toda, que aparece en la modelización de cristales, tiene la siguente expresión

$$
H=\frac{1}{2} \sum_{j=1}^{N} p_{j}^{2}+\sum_{j=1}^{N-1} e^{\left(q^{j+1}-q^{j}\right)}
$$


y cuando $N=3$

$$
H=\frac{1}{2}\left(p_{1}^{2}+p_{2}^{2}+p_{3}^{3}\right)+e^{q^{2}-q^{1}}+e^{q^{3}-q^{2}} .
$$

Es un ejercicio analítico fácil comprobar que cumple la condición de integrabilidad y obteniendo una primera integral lineal en los momentos conjugados como en (7.87) porque, como para la molécula de Toda, se cumple la condición

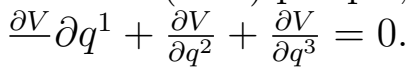

\section{Sistema Calogero-Moser general}

El sistema Calogeno-Moser describe el movimiento de $N$ partículas en una dimensión que se repelen con una fuerza proporcional al cubo de su distancia. EL hamiltoniano viene dado por

$$
H=\frac{1}{2} \sum_{j=1}^{N}\left(p_{n}^{2}+\omega^{2} q_{n}^{2}\right)+\epsilon^{2} \sum_{m, n=1 ; m \neq n}^{N}\left(q_{m}-q_{n}\right)^{-2} .
$$

Particularizando para $N=3$

$$
\begin{aligned}
& H=\frac{1}{2}\left(p_{1}^{2}+p_{2}^{2}+p_{3}^{2}\right)+ \\
& \frac{1}{2} \omega^{2}\left(q_{1}^{2}+q_{2}^{2}+q_{3}^{2}\right)+ \\
& \epsilon^{2}\left(\frac{1}{\left(q^{1}-q^{2}\right)^{2}}+\frac{1}{\left(q^{1}-q^{3}\right)^{2}}+\frac{1}{\left(q^{2}-q^{3}\right)^{2}}\right)
\end{aligned}
$$

La integrabilidad de este sistema es bien conocida y podemos comprobarla utilizando la condición de integrabilidad para cualquier valor de los parámetros $\omega, \epsilon$.

Además se cumple que

$$
\frac{\partial V}{\partial q_{1}}+\frac{\partial V}{\partial q_{2}}+\frac{\partial V}{\partial q_{3}}=\omega^{2}\left(q_{1}+q_{2}+q_{3}\right)
$$

por lo que para $\omega=0$ (el caso confinado) encontramos una integral primera lineal en los momenteos conjugados (7.87) como se describe en Rañada, 1999.

\section{Sistemas con potenciales centrales en 3D}

Vamos a analizar ahora una función hamiltoniana general con un potencial central del tipo

$$
H=\frac{1}{2}\left(p_{1}^{2}+p_{2}^{2}+p_{3}^{3}\right)+V\left(\left(q^{1}\right)^{2}+\left(q^{2}\right)^{2}+\left(q^{3}\right)^{2}\right) .
$$


Es fácil comprobar íticamente que estos hamiltonianos cumplen la condición de integrabilidad, ya que la relación

$$
\frac{\partial V}{\partial q^{1}}+\frac{\partial V}{\partial q^{2}}+\frac{\partial V}{\partial q^{3}}=\frac{\partial V}{\partial r} \frac{1}{r}\left(q^{1}+q^{2}+q^{3}\right)
$$

es análoga a (7.94), que corresponde al modelo anterior.

\section{Sistema de Choodnovzky 3D}

El modelo Choodnovzky viene descrito pr el siguiente hamiltoniano

$$
H=\frac{1}{2} \sum_{j=1}^{N} p_{j}^{2}+\frac{1}{2}\left(\sum_{j=1}^{N}\left(q^{i}\right)^{2}\right)^{2}-\sum_{i=1}^{N}\left(q^{i}\right)^{2} .
$$

Cuando $N=3$

$$
\begin{aligned}
H= & \frac{1}{2}\left(\left(p_{1}\right)^{2}+\left(p_{2}\right)^{2}+\left(p_{3}\right)^{2}\right)+ \\
& \frac{1}{2}\left(\left(q^{1}\right)^{2}+\left(q^{2}\right)^{2}+\left(q^{3}\right)^{2}\right)^{2}- \\
& \left(\left(q^{1}\right)^{2}+\left(q^{2}\right)^{2}+\left(q^{3}\right)^{2}\right)
\end{aligned}
$$

Es interesante comprobar que este sistema es integrable y cumple la condición de integrabilidad pero no la expresión (7.86).

\section{Generalización del sistema de Choodsnovzky 3D}

La generalizaciń de este sistema modeliza un conjunto de tres osciladores acoplados no lineales de cuarto grado, cuya función hamiltoniana es

$$
\begin{aligned}
& H=\frac{1}{2}\left(p_{1}^{2}+p_{2}^{2}+p_{3}^{3}\right)+\delta_{1}\left(q_{1}^{2}+q_{2}^{2}+q_{3}^{2}\right)+ \\
& 2 \alpha_{2}\left(\left(q_{2}-q_{1}\right)^{4}+\left(q_{3}-q_{1}\right)^{4}+\left(q_{3}-q_{2}\right)^{4}\right)+ \\
& \beta_{3}\left(q_{1}+q_{2}+q_{3}\right)^{4}
\end{aligned}
$$

Mediante la condición 7.79 podemos confirmar la integrabilidad para cualquier valor de sus parámetros reales $\delta_{1}, \alpha_{2}$ y $\beta_{3}$.

Igualmente podemos utilizar la condición de integrabilidad para sistemas no integrables a la hora de encontrar trayectorias confinadas en una variedad regular $U(\mathbf{q})=k$ como en el siguiente ejemplo

\section{Modelo de Hénon-Heiles 3D}

La generalización del modelo de Hénon-Heiles a tres grados de libertad viene dada por el suguiente hamiltoniano

$$
H=\frac{1}{2} \sum_{j=1}^{3} p_{j}^{2}+\frac{1}{2}\left(\sum_{j=1}^{3}\left(q^{i}\right)^{2}\right)+\left(\left(q^{1}\right)^{2}+\left(q^{2}\right)^{2}\right) q^{3}-\left(\frac{1}{3}\right)\left(q^{3}\right)^{3} .
$$


Este sistema posee un espacio de fases mixto, con grandes regiones de inestabilidad y caos, de forma que la condición de integrabilidad (7.79) no se cumple. Sin embargo hemos encontrado una condición más débil que si cumple estos sistema:

$$
-2\left(q^{1}-q^{2}\right) P\left(q^{i}\right)=0
$$

con $P\left(q^{i}\right)$ una expresión polinómica de segundo grado.

Esta condición se cumple si $q^{1}-q^{2}=0$ por lo que podemos encontrar una integral primera $I$ de la forma (7.75) definida en esta subvariedad

$$
\begin{array}{r}
I=\left(\left(q^{1}\right)^{2}+\left(q^{2}\right)^{2}-q^{2}-\left(q^{3}\right)^{2}+q^{3}-2 q^{2} q^{3}\right) p_{1} \\
-\left(\left(q^{1}\right)^{2}+\left(q^{2}\right)^{2}-q^{2}-\left(q^{3}\right)^{2}+q^{3}-2 q^{2} q^{3}\right) p_{2} \\
\left(q^{2}-q^{1}+2 q^{2} q^{3}-2 q^{1} q^{3}\right) p_{3}=0 .
\end{array}
$$

Considerando $q^{1}-q^{2}=0$ obtenemos la expresión

$$
I=\left(2\left(q^{1}\right)^{2}-q^{1}-\left(q^{3}\right)^{2}+q^{3}-2 q^{1} q^{3}\right)\left(p_{1}-p_{2}\right)=0 \Rightarrow p_{1}-p_{2}=0 .
$$

Con ello podemos encontrar una constante de movimiento lineal en los momentos conjugados de la forma dada en (7.11) con $C_{1}=1, C_{2}=-1$.

Analizando la condición de primera integral 7.75

$$
\begin{array}{r}
\{I(q, p), H(q, p)\}=\frac{\partial I}{\partial q^{i}} \frac{\partial H}{\partial p_{i}}-\frac{\partial I}{\partial p_{i}} \frac{\partial H}{\partial q^{i}}= \\
-q^{1}-2 q^{1} q^{3}+q^{2}+2 q^{2} q^{3} \underbrace{=}_{q^{1}=q^{2}} 0
\end{array}
$$

las ecuaciones de Hamilton vienen dadas por

$$
\left\{\begin{array}{l}
\frac{\partial H}{\partial q^{1}}=-\frac{d p_{1}}{d t}=q^{1}\left(1+2 q^{3}\right) \\
\frac{\partial H}{\partial q^{2}}=-\frac{d p_{2}}{d t}=q^{2}\left(1+2 q^{3}\right) \\
\frac{\partial H}{\partial q^{3}}=-\frac{d p_{3}}{d t}=\left(q^{1}\right)^{2}+\left(q^{2}\right)^{2}-\left(q^{3}\right)^{2} \\
\frac{\partial H}{\partial p_{k}}=\frac{d q^{k}}{d t}=p_{k} \quad k=1,2,3 .
\end{array}\right.
$$

Las trayectorias con condiciones iniciales en $U\left(q^{i}\right)=q_{0}^{1}-q_{0}^{2}=0$ con $p_{1}-p_{2}=0$ están confinadas en esta variedad $U(\mathbf{q})=0$ concluimos entonces que todas esas trayectorias no pueden alcanzar todas las regiones accesibles como se muestra en la Figura 7.2 .

\section{Método de estabilización para algunos sistemas Hamiltonianos}

Para terminar proponemos convertir la condición de integrabilidad (7.79) en una herramienta para estabilizar sistemas caóticos de la forma 7.82, para ello partiendo de un sistema hamiltoniano con dinámica caótica podríamos modificar el potencial como sigue

$$
V^{\prime}=V(\mathbf{q})+\sum_{i=1}^{n} a_{i} V_{i}(\mathbf{q})
$$



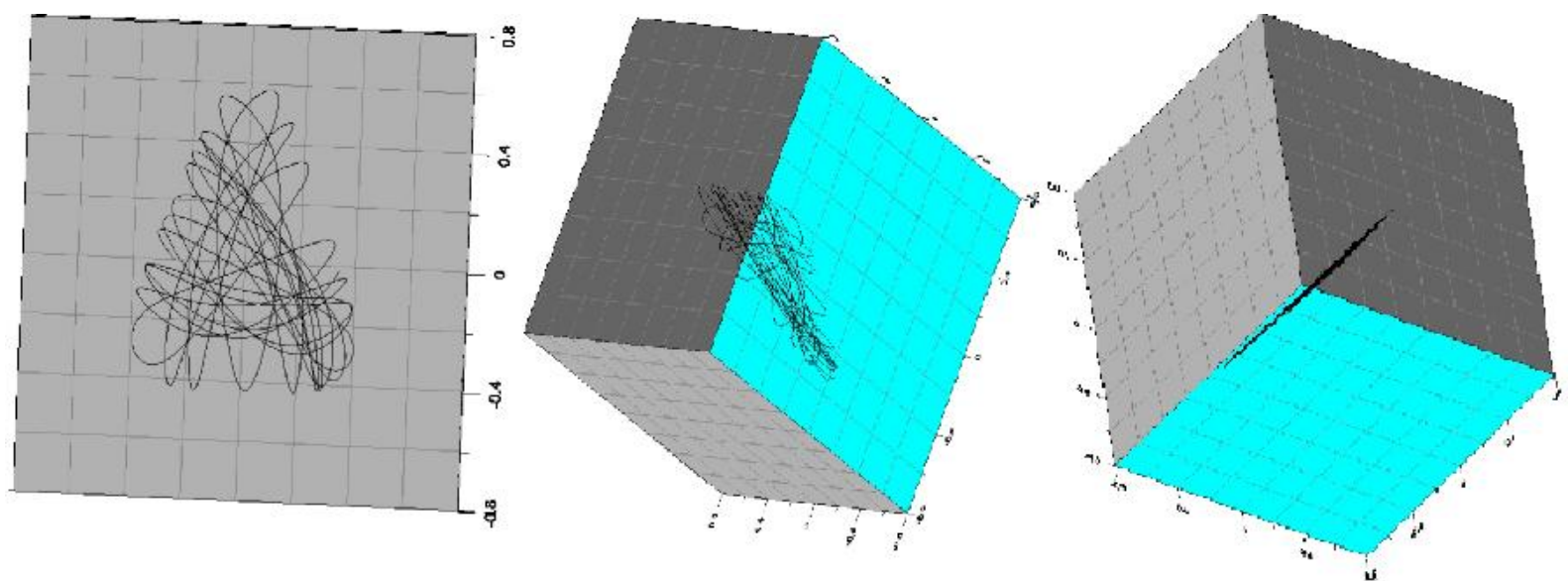

Figura 7.2: Diferentes perspectivas de una trayectoria caótica del sistema Hénon-Heiles 3D confinada en una subvariedad $U(\mathbf{q})=0$. Observese como desde la perspectiva de la figura derecha, la trayectoria parece regular

con $a_{i}$ parámetros arbitrarios, tal que imponiendo que el potencial $V^{\prime}$ cumpla las condición (7.79) obtendremos posibles valores de los parámetros $a_{i}$ para su integrabilidad y por tanto su estabilización. 



\section{Capítulo 8}

\section{Conclusiones}

En esta tesis hemos utilizado el formalismo geometrodinámico a través de la métrica de Jacobi, donde se describen los fenómenos físicos en términos de geometría diferencial, identificando las trayectorias con geodésicas de la variedad riemanniana definida con la métrica anterior.

A lo largo de la tesis hemos mostrado que este formalismo se puede utilizar para sistemas físicos de muy diferente escala, desde problemas cosmológicos, pasando por sistemas en los que rige la mecánica clásica, hasta problemas a escala cuántica donde, a partir de la reformulación geometrodinámica de la regla de cuantización de Einstein podemos obtener la ecuación de Schrödinger.

Nos hemos centrado en el estudio de sistemas conservativos hamiltonianos no lineales, mostrando cual es el origen del comportamiento caótico de estos sistemas desde la perspectiva del formalismo geometrodinámico y hemos definido nuevos indicadores geométricos de caos. Así mismo, hemos descrito un mecanismo novedoso de estabilización de trayectorias y encontrado un criterio suficiente de integrabilidad para sistemas tres dimensionales.

A continuación, se indican las conclusiones obtenidas en esta investigación:

1. Hemos definido un indicador de caos geométrico (SGI), que analiza la separación entre geodésicas en los sistemas hamiltonianos (análogo al exponente de Lyapunov en dinámica clásica, pero fijando la longitud de arco de las trayectorias, en vez del tiempo físico). Hemos mostrado que este indicador permite estudiar las zonas regulares y caóticas de sistemas hamiltonianos conservativos de dos grados de libertad.

2. Hemos implementado de forma exitosa la formulación geometrodinámica al sistema molecular LiNC-LiCN con dos grados de libertad, definido a través de coordenadas de Jacobi. A partir de su matriz de masa hemos 
determinado la métrica de Jacobi, caracterizando las geodésicas y encontrando la expresión exacta de la curvatura escalar en función de la energía cinética y de las derivadas del potencial

3. El indicador geométrico SGI nos ha permitido estudiar la estructura del espacio de fases del sistema molecular LiNC-LiCN con dos grados de libertad. Hemos comprobado cómo el valor del SGI en este sistema permanece constante para órbitas periódicas, crece linealmente para órbitas cuasiperiódicas, con una pendiente mayor cuanto más se aleja de la órbita periódica, y crece exponencialmente para trayectorias caóticas.

4. Así mismo, el indicador es suficientemente sensible como para detectar las estructuras subyacentes del espacio de fases, como es la presencia de un cantoro en la frontera entre la zona regular y caótica del espacio de fases en este sistema a energías intermedias.

5. La descripción geometrodinámica nos ha permitido analizar el efecto de la curvatura de la frontera del espacio accesible a una determinada energía (denominado región de Hill). Hemos encontrado trayectorias caóticas cuyos puntos de retorno son todos sobre la zona de curvatura positiva en la frontera y trayectorias regulares con puntos de retorno sobre zonas de curvatura negativa. Esto implica que el sistema molecular LiNC-LiCN con dos grados de libertad no puede reducirse a un problema de billar y que el origen del caos en este sistema está en la presencia de resonancias paramétricas, donde la curvatura escalar del espacio parece jugar un papel fundamental en la estabilización de las trayectorias.

6. Partiendo de la idea de que, en un campo de fuerzas, el arco más estable es el arco catenario (arco cuya tensión acumulada a lo largo de su longitud es mínima), hemos definido un nuevo indicador que estudia la distancia entre las geodésicas de un sistema a sus curvas catenarias. Este indicador es independiente de los grados de libertad del sistema, si bien es necesario que la matriz de masas sea la identidad en sistemas de más de dos grados de libertad, es decir que esté definido en coordenadas cartesianas.

7. Hemos estudiado la distancia a la catenaria en el sistema Hénon-Heiles, comprobando que el análisis de la fluctuación de la media móvil de la distancia normalizada a la geodésica es un indicador que permite obtener la estructura del espacio de fases de este sistema, detectando las zonas de regularidad y caos de forma muy precisa. El valor del indicador catenario es varios órdenes de magnitud mayor en el caso de órbitas caóticas que para órbitas regulares.

8. Esta analogía entre catenarias y órbitas estables nos ha permitido desarrollar un criterio de integrabilidad: si es posible foliar todo el espacio 
por superficies formadas por trayectorias catenarias, es decir estables, el sistema será integrable. En ocasiones la condición catenaria para una trayectoria implica la existencia de un campo vectorial Killing asociado a simetrías del sistema.

9. Hemos podido obtener de forma analítica un criterio suficiente de integrabilidad para sistemas de tres grados de libertad.

10. Hemos podido mostrar la integrabilidad de diferentes sistemas hamiltonanos de tres grados de libertad comprobando que cumplen el criterio propuesto.

Como trabajo futuro sería tremendamente interesante estudiar el mecanismo estabilizador de la curvatura del espacio subyacente para sistemas más generales, ampliar el criterio de integrabilidad estudiado a sistemas con matriz de masa no reducible a la identidad, estudiar el criterio anterior para sistemas de más de tres grados de libertad, estudiar el efecto de los puntos conjugados para determinar la regularidad o no de las trayectorias, etc.. 



\section{Bibliografía}

[Arnold, 1963] V. I. Arnold. Proof of a theorem of a. N. Kolmogorov on the invariance of quasi-periodic motions under small perturbations of the Hamiltonian. Rus. Math. Surv. 18 (5), 9-36 (1963). https://doi.org/10.1070/RM1963v018n05ABEH004130

[Arranz et al., 2000] F. J. Arranz, F. Borondo, and R. M. Benito. Transition from order to chaos in a floppy molecule: LiNC/LiCN. Chem. Phys. Lett. 317 (3-5), 451-457 (2000). https://doi.org/10.1016/S00092614(99)01426-8

[Arranz, 2013] F. J. Arranz, L. Seidel, C. G. Giralda, R. M. Benito, and F. Borondo. Onset of quantum chaos in molecular systems and the zeros of the Husimi function. Phys. Rev. E 87, 062901 (2013) https://doi.org/10.1103/PhysRevE.87.062901

[Bari et Cipriani, 1998] M. Bari and P. Cipriani. Geometry and Chaos on Riemann and Finsler Manifolds. Planetary and Space Science,46, 1543 (1998) http://dx.doi.org/10.1016/S0032-0633(98)00059-2

[Barrio, 2005] R. Barrio. Sensitivity Tools vs. Poincare Sections. Chaos, Solitons and Fractals 25, 711 (2005). http://dx.doi.org/10.1016/j.chaos.2004.11.092

[Barrow-Green, 1997] J. Barrow-Green. Poincaré and the Three Body Problem. American Mathematical Society, (1997). ISBN-13 : 9780821803677

[Beltrami] E. Beltrami. Teoria fondamentale degli spazii di curvatura costante. Annali di Matematica Pura ed Applicata, 2 (1), 232-255.(1868)

[Ben Zion et Horwitz, 2007-] Y. Ben Zion and L. Horwitz. Detecting order and chaos in three-dimensional Hamiltonian systems by geometrical methods. Phys. Rev. E 76, 046220 (2007). https://doi.org/10.1103/PhysRevE.76.046220; Controlling effect of geometrically defined local structural changes on chaotic Hamiltonian systems. 81, 046217 (2010). https://doi.org/10.1103/PhysRevE.81.046217 
[Ben Zion et Horwitz, 2008] Y. Ben Zion and L. Horwitz. Applications of geometrical criteria for transition to Hamiltonian chaos. 78, 036209 (2008). https://doi.org/10.1103/PhysRevE.78.036209

[Benítez et al., 2013] P. Benítez, J.C. Losada, R.M. Benito, and F. Borondo. Analysis of the Full Vibrational Dynamics of the LiNC/LiCN Molecular System. Progress and Challenges in Dynamical Systems 54, 77-88 (2013). http://dx.doi.org/10.1007/978-3-642-38830-9-6

[Benito et al., 1989] R. M. Benito, F. Borondo, J.-H Kim, B.G. Sumpter, and G.S. Ezra. Comparison of classical and quantum phase space structure of nonrigid molecules, LiCN. Chem. Phys. Lett. 161, 60 (1989). https://doi.org/10.1016/S0009-2614(89)87032-0

[Bernoulli, 1742] D. Bernoulli. Disquisitio Catoptico-Dioptrica. Opera Omnia (Geneve, sumptibus Marci-Michaelis Bousquet \& sociorum, Vol. I pp. 369-376 (1742).

[Berry, 1978] M. V. Berry. Regular and irregular motion. AIP Conf. Proc. 46, 16 (1978). https://doi.org/10.1063/1.31417

[Birkhoff, 1931] G. D. Birkhoff. Une généralisation à n dimensions du dernier thórème de gómétrie de Poincaré. Comptes Rendus Acad. Sci. 192, 196 (1931).

[Bolyai, 1832] J. Bolyai. Scientiam Spatii absolute veram exhibens (The absolute true science of space). Apéndice del libro Tentamen Juventutem studiosam en elementa Matheseos purae, elementaris sublimioris de corriente alterna, methodo intuitiva, evidentiaque Huic propria, introducendi. (An Attempt to Introduce Studious Youths to the Elements of Pure Mathematics). F. Bolyai. Typis Collegii Reformatorum (1832). https://library.si.edu/digital-library/book/tentamenjuventu2boly

[Borondo et al., 1995] F. Borondo, A. A. Zembekov and R. M. Benito. Quantum manifestations of saddle-node bifurcations. J. Chem. Phys. Lett. 246, 421 (1995). https://doi.org/10.1016/0009-2614(95)01147-X

[Borondo et al., 1996] F. Borondo, A. A. Zembekov, R. M. Benito. Saddleânode bifurcations in the $\mathrm{LiNC} / \mathrm{LiCN}$ molecular system: Classical aspects and quantum manifestations. J. Chem. Phys. 105; 5068 (1996). https://doi.org/10.1063/1.472351

[Borondo et al., 1997] F. Borondo, A. A. Zembekov and R. M. Benito. Semiclassical quantization of fragmented tori: Application to saddlenode states of LiNC/LiCN. J. Chem. Phys. 107; 7934 (1997). https://doi.org/10.1063/1.475147 
[Casetti et al., 1993] L. Casetti and M. Pettini. Analytic computation of the strong stochasticity threshold in Hamiltonian dynamics using Riemannian geometry. Phys. Rev. E 48, 4320 (1993). https://doi.org/10.1103/PhysRevE.48.4320

[Casetti et al., 1995] L. Casetti, R. Livi, and M. Pettini. Gaussian Model for Chaotic Instability of Hamiltonian Flows. Phys. Rev. Lett. 74, 375 (1995). https://doi.org/10.1103/PhysRevLett.74.375

[Casetti et al., 1996] L. Casetti, C. Clementi, and M. Pettini. Riemannian Theory of Hamiltonian Chaos and Lyapunov Exponents. hys. Rev. E 54, 5969 (1996). http://dx.doi.org/10.1103/PhysRevE.54.5969

[Casetti et al., 2000] L. Casetti, M. Pettini, and E.G.D Cohen. Geometric Approach to Hamiltonian Dynamics and Statistical Mechanics. Phys. Rep. 337, 237-241 (2000). http://dx.doi.org/10.1016/S0370-1573(00)00069-7

[Cerruti-Sola et Pettini, 1995] M. Cerruti-Sola and M. Pettini. Geometric Description of Chaos in Self-Gravitating Systems. Phys. Rev. E 51, 53 (1995). http://dx.doi.org/10.1103/PhysRevE.51.53

[Cerruti-Sola et Pettini, 1996] M. Cerruti-Sola and M. Pettini. Geometric Description of Chaos in Two-Degrees-ofFreedom Hamiltonian Systems. Phys. Rev. E 53, 179 (1996). http://dx.doi.org/10.1103/PhysRevE.53.179

[Cincotta et al., 2003] P. M. Cincotta, C. M. Giordano, and C. Simó. Phase space structure of multi-dimensional systems by means of the mean exponential growth factor of nearby orbits. Physica D, 182151 (2003). https://doi.org/10.1016/S0167-2789(03)00103-

[Cincotta et Simó, 2000] P. M. Cincotta and C. Simó. Simple tools to study global dynamics in non-axisymmetric galactic potentials â I. Astron. Astrophys. Suppl. Ser. 547, 205-228 (2000). https://doi.org/10.1051/aas:2000108

[Cipriani et Bari, 1998a] P. Cipriani, and M. Bari. Finsler Geometric Local Indicator of Chaos for Single Orbits in the Henon-Heiles Hamiltonian. Phys. Rev. Lett. 81, 5532 (1998). http://dx.doi.org/10.1103/PhysRevLett.81.5532

[Cipriani et Bari, 1998b] P. Cipriani and M. Bari. Geometry and chaos on Riemann and Finsler manifolds. Planet. Space Sci. 46, 1543 (1998). https://doi.org/10.1016/S0032-0633(98)00059-2 
[Dai et Field, 1995] H. L. Dai and R. W. Field (editors), Molecular Dynamics and Spectroscopy by SEP (World Scientific, Singapore, 1995).

[Di Bari et al., 1997 ] M. Di Bari, D. Boccaletti, P. Cipriani, and G. Pucacco. Dynamical behavior of Lagrangian systems on Finsler manifolds. Phys. Rev. E 55, 6448 (1997). https://doi.org/10.1103/PhysRevE.55.6448

[Di Cairano et al., 2019] L. Di Cairano, M. Gori and M. Pettini. Coherent Riemannian-geometric description of Hamiltonian order and chaos with Jacobi metric. Chaos 29, 123134 (2019). http://doi.org/10.1063/1.5119797

[do Carmo, 1976] M. P. Carmo, Differential Geometry of Curves and Surfaces (Prentice Hall, Englewood Cliffs, 1976).

[do Carmo, 1992] M. P. do Carmo, (1992) Riemannian Geometry (Birkhauser, Boston, 1992).

[Dumas et Laskar, 1993] H. S. Dumas and J. Laskar. Global Dynamics and Long-Time Stability in Hamiltonian Systems via Numerical Frequency Analysis. Phys. Rev. Lett. 70 2975, (1993). https://doi.org/10.1103/PhysRevLett.70.2975

[Einstein, 1905] A. Einstein. Uber einen die Erzeugung und Verwandlung des Lichtes betreffenden heuristischen Gesichtspunkt (On a Heuristic Point of View Concerning the Production and Transformation of Light). Annalen der Physik (ser. 4), 17, 132-148 (1905). http://myweb.rz.uni-augsburg.de/ eckern/adp/history/einstein-papers /1905_17_132-148.pdf; Para una versión en inglés, ver por ejemplo: https://www.pitt.edu/ jdnorton/lectures/Rotman_Summer_School_2013/ Einstein_1905_docs/Einstein_Light_Quantum_WikiSource.pdf

[Einstein, 1915-16] A. Einstein. Fundamental Ideas of the General Theory of Relativity and the Application of this Theory in Astronomy. Preussische Akademie der Wissenschaften, Sitzungsberichte, (parte 1) 315; (parte 2) 778-786, 799-801; (parte 3) 831-839; (parte 4) 844847. (1915); The Foundation of the General Theory of Relativity. Annalen der Physik (4), 49, 769-822 (1916). http://myweb.rz.uniaugsburg.de/ eckern/adp/history/einstein-papers/1916_49_769-822.pdf

[Eisenhart, 1929] Eisenhart, L.P. Dynamical Trajectories and Geodesics. Annals of Mathematics 30, 591 (1929). http://dx.doi.org/10.2307/1968307

[Esser et al., 1982] R. Esser, J. Tennyson and P. E. S. Wormer. An SCF potential energy surface for lithium cyanide. Chem. Phys. Lett. 89, 223 (1982). https://doi.org/10.1016/0009-2614(82)80046-8 
[Eyring et Polanyi, 1931] H. Eyring, and M. Polanyi. Z. Phys Chem. (Leipzig) B12, 279 (1931)

[Fukui, 1970] K. Fukui. Formulation of the reaction coordinate. J. Phys. Chem. 74, 4161 (1970). https://doi.org/10.1021/j100717a029

[Gauss, 1843] C.F. Gauss . Investigaciones sobre objetos de geodesia superior. Primera disertación., Disertaciones de la Sociedad Real de las Ciencias en Gotinga. Segundo tomo., pp. 3-46 (1843-33); Investigaciones sobre objetos de geodesia superior. Segunda disertación., Disertaciones de la Sociedad Real de las Ciencias en Gotinga. Tercer tomo. pp. 3-44.(184647)

[Geisel et al., 1986] T. Geisel, G. Radons, and J. Rubner. Kolmogorov-Arnol'd-Moser Barriers in the Quantum Dynamics of Chaotic Systems. Phys. Rev. Lett. 57, 2883 (1986). https://doi.org/10.1103/PhysRevLett.57.2883; Erratum Phys. Rev. Lett. 58, 2506 (1987). https://doi.org/10.1103/PhysRevLett.58.2506

[Henon et Heiles, 1964] M. Henon and C. Heiles. The applicability of the third integral of motion: Some numerical experiments. Astron. J. 69, 73 (1964). https://doi.org/10.1086/109234

[Horwitz et al., 2007] L. Horwitz, Y.B. Zion, M. Lewkowics, M. Schiffer, and J. Levitan. Geometry of Hamiltonian Chaos. Phys. Rev. Lett. 98, 234301 (2007). http://dx.doi.org/10.1103/PhysRevLett.98.234301

[Iraq tourists, 2013] https://www.thejournal.ie/iraq-tourists-arch-ofctesiphon-931394-Jun2013 (Figure 1)

[Joy et Sabir, 1993] M. P. Joy, M. Sabir. Chaos and curvature in a quartic Hamiltonian system. Pramana - J Phys 40, $17 \hat{a} 23$ (1993). https://doi.org/10.1007/BF02898037

[Kassel, 1928] L.S. Kassel. Studies in Homogeneous Gas Reactions I. The Journal of Physical Chemistry, 32 (2): 225â242, (1928). https://doi.org/10.1021/j150284a00

[Klein, 1872] F. Klein. Vergleichende Betrachtungen Ã $\frac{1}{4}$ ber neuere geometrische Forschungen. (A comparative review of recent researches in geometry). Programme on entering the philosophical faculty and the senate of the university of Erlangen in 1872. (1872). https://arxiv.org/pdf/0807.3161.pdf 
[Kosloff et Rice, 1981] R. Kosloff and S. A. Rice. Dynamical correlations and chaos in classical Hamiltonian systems. J. Chem. Phys. 74, 1947 (1981). https://doi.org/10.1063/1.441259

[Lega, 2003] E. Lega, M. Guzzo, and C. Froeschlé, Detection of Arnold Diffusion in Hamiltonian Systems. Physica D 182, 179 (2003). http://dx.doi.org/10.1016/S0167-2789(03)00121-0

[Lobachevski, 1829] N.I. Lobachevski. On the Principles of Geometry. Kasan Bulletin Vol. 1 pp 1-67. (1829-1830)

[Lorenzt, 1904] H. A. Lorenzt. Electromagnetic phenomena in a system moving with any velocity smaller than that of light, Proc. of the Royal Netherlands Academy of Arts and Sciences,6: $809 \hat{a} 83$ (1904). https://www.dwc.knaw.nl/DL/publications/PU00014148.pdf

[Losada et al., 1998] Losada, J.C., Estebaranz, J.M., Benito, R.M. and Borondo, F. Local Frequency Analysis and the Structure of Classical Phase Space of the LiNC/LiCN Molecular System. J. Chem. Phys. 108, 63 (1998). http://dx.doi.org/10.1063/1.475365

[Marcus, 1953] R. A. Marcus. Unimolecular Dissociations and Free Radical Recombination Reactions., J. Chem. Phys., 20 (3): 359â364 (1952) doi:10.1063/1.1700424

[Maupertuis, 1940-] P.L. Maupertuis. La Loi du repos des corps (La ley de los cuerpos en reposo). Mémoires de l'Académie Royale des Sciences Année 1740 pp. $170 a ̂ 176$ (1742); Accord de plusieurs lois naturelles qui avaient paru jusqu'ici incompatibles (Acuerdo de varias leyes naturales que hasta ahora parecÃan incompatibles). Mémoires de l'Académie Royale des Sciences Année 1744 pp. 417â426 (1747); Loix du mouvement et du repos (Leyes del movimiento y el descanso). Mémoires de Académie Royale des Sciences et des Belles Lettres de Berlin Année 1746, p. 267-294 (1748),

[Maxwell, 1873] J. C. Maxwell. A Treatise on Electricity and Magnetism. Oxford University Clarendon Press (1873). https://doi.org/10.1017/CBO9780511709340

[Meckler, 1976] A. Meckler. The hanging string as a model of metric distorsion. Am. J. Phys. 44, (1976). https://doi.org/10.1119/1.10135

[Michelson, 1881] A.Michelson. The relative motion of the Earth and the luminiferous ether. American Journal of Science, 22, p. 120-9, (1881). https://physics.ucf.edu/ rep/EDI/michelson.pdf 
[Minkowki, 1908] H. Minkowki. Space and Time. Discurso de inauguración de la 80 reunión de la Asamblea general alemana de científicos naturales y físicos. 21 de septiembre de 1908 (1908). https://www.minkowskiinstitute.org/mip/MinkowskiFreemiumMIP2012. pdf

[Newton, 1686] I.S. Newton. PhilosophiA $\tilde{A}_{1}^{\prime}$ naturalis principia mathematica. S. Pepys, Reg. Soc. Press (1686) http://cudl.lib.cam.ac.uk/view/PR-ADVB-00039-00001/

[Numerical Recipes, 1996] [Numerical Recipes]. Numerical Recipes in Fortran 90. The Art of Parallel Scientific Computing, 2nd Edition, 1996, ISBN 0-521-57439-0. http://numerical.recipes/

[Pettini, 1993] M. Pettini. Geometrical hints for a nonperturbative approach to Hamiltonian dynamics. Phys. Rev. E 47, 828 (1993). https://doi.org/10.1103/PhysRevE.47.828

[Planck, 1900] M. Planck. On the distribution law of energy in the normal spectrum. German Physical Society Meeting on October 19 and December 14, 1900, proceedings 2, p. 202 and p. 237, (1900); Annalen der Physik IV, Folge 4, 553-563 (1901). http://web.pdx.edu/ pmoeck/pdf/planckpaper.pdf

[Quapp et Heidrich, 1984] W. Quap, D. Heidrich. Analysis of the concept of minimun energy path on the potential energy surface of chemically reacting systems. Theoret. Chim. Acta 66, 245-260 (1984). https://doi.org/10.1007/BF00549673

[Rañada, 1999] M. F. Rañada. Superintegrability of the Clogero-Moser sistem: constants of motion, master symmetries, and time-dependent symmetries. Journal of Mathematical Physics 40, 236 (1999). https://doi.org/10.1063/1.532770

[Ren et Vanden-Eijnden, 2002] W. E, W. Ren, and E. Vanden-Eijnden. String method for the study of rare events. Phys. Rev. B 66, 052301 (2002). https://doi.org/10.1103/PhysRevB.66.052301

[Riemann] B. Riemann. Ueber die Hypothesen, Welche der Geometrie zu Grunde liegen (Sobre las hipótesis en que se funda la geometría). Gotinga (1854). Clase magistral impartida por Riemann en Gotinga en 1854 a petición de Gauss; Tratados de la Real Sociedad de Ciencias de Gottingen 13 (1868), pp. 133-150. 
[Rice and Ramsperger, 1927] O. K. Rice, H.C. Ramsperger. Theories of unimolecular gas reactions at low pressures. Journal of the American Chemical Society, 49 (7): $1617 \hat{1} 1629$ (1927). https://doi.org/10.1021/ja01406a001

[Ricci et al., 2016] E.Ricci, E. Sacco and M.D.Piccioni A method for the analysis of masonry arches (fig. 3). Conference Paper: 10th. International Conference on Structural Analysis of historical Constructions. SAHC. At. Leuven, Belgio. September 2016

[Rojo, 2005] A. G. Rojo, Hamiltonâs principle: Why is the integrated difference of the kinetic and potential energy minimized? Am. J. Phys. 73, 831 (2005) . https://doi.org/10.1119/1.1930887

[Saa, 2004] A. Saa. On the Viability of Local Criteria for Chaos. Annals of Physics, 314 508-516 (2004). http://dx.doi.org/10.1016/j.aop.2004.08.008

[Schinke, 1992] R. Schinke, Photodissociation Dynamics (Cambridge University Press, Cambridge, 1992).

[Simó, 1999] C. Simó (ed.), Hamiltonian Systems with Three or More Degrees of Freedom NATO ASI Series C, vol. 533 (Kluwer, Dordrecht, 1999); S. Ibáñez et. al. (eds.), Progress and Challenges in Dynamical Systems Springer Proceedings in Mathematics \& Statistics vol. 54 (SpringerVerlag, Berlin, 2013).

[Skokos et al., 2004] Ch. Skokos, Ch. Antonopoulos, T.C. Bountis, and M.N. Vrahatis, Detecting Order and Chaos in Hamiltonian Systems by the SALI Method. J.Phys. A 37, 6269 (2004). http://dx.doi.org/10.1088/0305$4470 / 37 / 24 / 006$

[Skokos, 2010] Ch. Skokos, The Lyapunov Characteristic Exponents and Their Computation. Lecture Notes in Physics 790, 63 (2010). http://dx.doi.org/10.1007/978-3-642-04458-8_2

[Toda, 1974] M. Toda. Instability of trajectories of the lattice with cubic nonlinearity. Phys. Lett. A 48, 335 (1974). https://doi.org/10.1016/03759601(74)90454-X

[Vergel et al., 2014a] A. Vergel, J. C. Losada, R. M. Benito, F. Borondo. Geometrodynamical Analysis to Characterize the Dynamics and Stability of a Molecular System through the Boundary of the Hillâs Region. Applied Mathematics 5, 49878 (2014). http://dx.doi.org/10.4236/am.2014.516251 
[Vergel et al., 2014b] A. Vergel, R. M. Benito, J. C. Losada, F. Borondo. Geometrical Analysis of the LICN Vibrational Dynamics. A Stability Geometrical Indicator. Phys. Rev. E 89, 022901 (2014). http://dx.doi.org/10.1103/PhysRevE.89.022901

[Weinan et al., 2007] E. Weinan, R. Weiqing and E. Vanden-Eijnden. Simplified and improved string method for computing the minimum energy paths in barrier-crossing events. J. Chem. Phys. 126, 164103 (2007). http://dx.doi.org/10.1063/1.2720838

[Wisniacki et al., 2011] D. A. Wisniacki, M. Saraceno, F. J. Arranz, R. M. Benito, and F. Borondo. PoincarÃ (C)-Birkhoff theorem in quantum mechanics. Phys. Rev. E 84, 026206 (2011). https://doi.org/10.1103/PhysRevE.84.026206

[Zewail, 1992] A. Zewail. (ed.), The Chemical Bond: Structure and Dynamics (Academic Press, San Diego, 1992).

[Zhu et al., 2019] X. Zhu, K. C, Thompson, and T. J. Martínez. Geodesic interpolation for reactive pathways. J. Chem. Phys. 150, 164103 (2019). https://doi.org/10.1063/1.5090303

[Zion et Horwitz, 2008] Y. B. Zion and L. Horwitz. Applications of Geometrical Criteria for Transition to Hamiltonian Chaos. Phys. Rev. E, 78, 036209 (2008). https://doi.org/10.1103/PhysRevE.78.036209 

APÉNDICES 



\section{Apéndice A}

\section{Otras aplicaciones: cuantización}

\section{A.1. Métrica de Jacobi y regla de cuantización de Einstein}

En 1917 A. Einstein introduce una regla de cuantización que permite establecer la conexión entre la teoría cuántica y las ondas de materia de De Broglie. Nosotros emplearemos dicha regla para mostrar cómo la geometría riemanniana, en particular, la generada a partir de la métrica Jacobi surge de forma natural y es fuente de nuevas intuiciones dentro de este contexto geométrico. Además, observamos que las funciones de onda obtenidas de la resolución de la ecuación de Schrödinger pueden reproducirse al resolver la ecuación de JLC en la variedad mecánica (métrica Jacobi).

La Regla de Einstein se muestra como una formulación de la condición de cuantización invariante ante cambios de coordenadas.

Según De Broglie, la regla dice

$$
\oint p_{i} d q^{i}=n h
$$

realizando la integral a lo largo de cualquier curva cerrada en el espacio de configuración del sistema y con $n \in \mathbb{Z}$.

El resultado de A.1 será el mismo para todas las curvas cerradas que puedan ser deformadas continuamente entre ellas.

Para las curvas que, mediante transformación continua, puedan reducirse a un punto, el valor de la integral será cero. 
La anterior condición debe cumplir

$$
\delta \oint p_{i} d q^{i}=0
$$

que equivale a la aplicación del Principio de Maupertuis sobre trayectorias isoenergéticas del sistema dinámico, de ahí que de Broglie afirme que la integral de acción desempeñe un papel importante tanto en mecánica clásica (Principio de Maupertuis) como en teoría cuántica ( regla de cuantización de Einstein).

La conexión entre las teorías anteriores vía integral de acción puede verse fácilmente de la siguiente manera

$$
\begin{aligned}
& n h=\oint p_{i} d q^{i}=\oint_{\gamma(t)} \frac{\partial L}{\partial \dot{q}^{i}} \dot{q}^{i} d t= \\
& \oint_{\gamma(t)} 2 W d t=\oint_{\gamma(t)} \sqrt{2(E-V(\mathbf{q})) a_{i j}(\mathbf{q}) \dot{q}^{i} \dot{q}^{j}} d t=\oint_{\gamma(s)} d s=L,
\end{aligned}
$$

con $L=$ longitud de la curva cerrada $\gamma(t)$ medida con la métrica de Jacobi y $n \in \mathbb{Z}$. Se tiene entonces que

$$
\begin{gathered}
n h=\oint_{\gamma(s)} d s=L \text { con } h=2 \pi \hbar(\hbar \equiv \text { constante Planck reducida }) \\
n h=L \text { con } h=2 \pi \hbar
\end{gathered}
$$

\section{A.1.1. Cuantización del momento angular}

$$
n h=\oint 2 W d t=\oint \sqrt{2 W} \sqrt{2 W} d t=\oint v \sqrt{a_{i j}(\mathbf{q}) \dot{q}^{i} \dot{q}^{j}} d t=\oint v d l
$$

$d l \equiv$ diferencial de longitud medida con métrica de vacío $a_{i j}(\mathbf{q})$.

Si el espacio sin campo físico representado por la matriz $a_{i j}(\mathbf{q})$ es euclídeo entonces se cumple

$$
n \hbar=v r
$$

\section{A.2. Aplicación: el átomo hidrogenoide}

Tomemos el potencial en coordenadas polares de la forma

$$
V(r)=\frac{-K}{r} .
$$


La métrica Jacobi toma la forma

$$
g_{i j}=2(E-V(\mathbf{q})) a_{i j}(\mathbf{q})=2\left(E+\frac{k}{r}\right)\left(\begin{array}{cc}
1 & 0 \\
0 & r^{2}
\end{array}\right) .
$$

Calculamos la curvatura escalar de la variedad mecánica

$$
\mathcal{R}=\frac{-E K}{2(r E+K)^{3}} .
$$

Podemos observar que la curvatura escalar es no singular para $r=0$, esto es, $\mathcal{R}(0)=\frac{-E}{2 K^{2}}$. Por tanto, y dado que la curvatura escalar es un invariante geométrico y no depende del sistema coordenado podemos deducir que el origen de coordenadas no es un punto singular del sistema aunque no esté definido cuando utilizamos coordenadas polares. En cambio, la singularidad aparece en $r E+K=0$, esto es, en los puntos de retorno del sistema. Desde el punto de vista geométrico solamente hay un punto de retorno ya que si $V(r) \approx E+\epsilon$ entonces como $g_{i j}=2(E-V(r)) a_{i j} \approx \epsilon a_{i j}$ y la distancia entre los diferentes puntos de retorno sería del orden de $\epsilon$.

Utilizaremos el Principio de cuantización/conservación topológica: para toda trayectoria cerrada, se cumple

$$
\int_{R} \frac{\mathcal{R}}{2} d \sigma=-K \int_{0}^{2 \pi} \int_{\epsilon}^{r=c t e}(r E+K)^{-2} E d r d \theta=2 \pi \chi(R)=2 \pi n, \text { con } n \in \mathbb{Z},
$$

siendo $d \sigma=\sqrt{|g|} d r d \theta=2\left(E+\frac{K}{r}\right) r d r d \theta=2(E r+K) d r d \theta$ el diferencial de área y haciendo $\epsilon \longrightarrow 0$ queda

$$
\frac{-E r}{E r+K}=n \Rightarrow r=\frac{-K n}{E(1+n)}, \text { con } n \in \mathbb{Z}
$$

Por tanto, energía y distancia son magnitudes inversas, esto es $E r=-\frac{k n}{1+n}$. Luego en el caso del átomo hidrogenoide, las órbitas más próximas al núcleo son las más energéticas.

Por sustitución directa en las ecuaciones geodésicas, encontramos que la única geodésica de radio constante $r=$ constante tiene la forma $r=-\frac{K}{2 E}$. Por tanto, igualando ambas expresiones podemos deducir la característica de Euler de la región $R$ limitada por la curva $R=\frac{-K}{2 E}$

$$
\frac{-K n}{E(1+n)}=-\frac{K}{2 E} \Rightarrow n=1=\chi(R) .
$$

Podemos encontrar entonces la topología de la región $R$ limitada por la curva $r=\frac{-K}{2 E}$. 
Para ello, sustituimos en la expresión A.10 para $r=\frac{-k}{2 E}$ y obtenemos

$$
\mathcal{R}=\frac{-2 E}{K^{2}}
$$

Aplicando la regla de cuantización de Einstein A.7 tomamos la curva cerrada $\gamma(t)=\left(-\frac{K}{2 E}, t\right) \Rightarrow \dot{\gamma}(t)=(0,1)$ y calculamos su longitud $L$

$$
\begin{gathered}
\|\dot{\gamma}\|^{2}=\left(\begin{array}{ll}
0 & 1
\end{array}\right)\left(\begin{array}{cc}
2\left(E+\frac{k}{r}\right) & 0 \\
0 & 2\left(E+\frac{k}{r}\right) r^{2}
\end{array}\right)\left(\begin{array}{l}
0 \\
1
\end{array}\right)= \\
\left(\begin{array}{ll}
0 & 1
\end{array}\right)\left(\begin{array}{cc}
-2 E & 0 \\
0 & -\frac{K^{2}}{2 E}
\end{array}\right)\left(\begin{array}{l}
0 \\
1
\end{array}\right) \Rightarrow \\
\|\dot{\gamma}\|=\sqrt{-\frac{K^{2}}{2 E}}
\end{gathered}
$$

Por tanto, la longitud total será

$$
L=\oint\|\dot{\gamma}(t)\| d t=\oint \sqrt{-\frac{K^{2}}{2 E}} d t=\sqrt{-\frac{K^{2}}{2 E}} 2 \pi,
$$

por lo que la regla de cuantización dice

$$
\sqrt{-\frac{K^{2}}{2 E}} 2 \pi=n h, \quad \text { con } \quad h=2 \pi \hbar .
$$

Despejando la energía $E$, se tiene

$$
E_{n}=\frac{-K^{2}}{2 n^{2} \hbar^{2}}
$$

Para el caso particular de un átomo hidrogenoide

$$
V(r)=-\frac{K}{r}=-\frac{Z e^{2}}{4 \pi \epsilon_{0}} \frac{1}{r} \Rightarrow K=\frac{Z e^{2}}{4 \pi \epsilon_{0}},
$$

luego las energías permitidas serán

$$
E_{n}=-\left(\frac{Z^{2} e^{2}}{4 \pi \epsilon_{0}}\right)^{2} \frac{1}{2 n^{2} \hbar^{2}}=-\frac{Z^{2} e^{4}}{\left(4 \pi \epsilon_{0}\right)^{2} 2 n^{2} \hbar^{2}} .
$$

Ahora podemos calcular los radios orbitales como

$$
r_{n}=\frac{-K}{2 E_{n}} \Rightarrow r_{n}=\frac{n^{2} \hbar^{2}}{K}=\frac{n^{2}}{Z} a_{0}, \quad \text { con } a_{0}=\frac{\epsilon_{0} h}{m_{e} e^{2} \pi} \equiv \quad \text { Radio de Bohr. }
$$




\section{A.2.1. Estudio de estabilidad. Estado fundamental}

Estudiemos la ecuación de Jacobi-Levi-Civita

$$
\frac{d^{2} \psi}{d s^{2}}+\frac{\mathcal{R}}{2} \psi=0, \quad \text { con } \mathcal{R} \equiv \text { curvatura escalar. }
$$

En el caso del átomo hidrogenoide y $E=\frac{V(\mathbf{q})}{2}=-\frac{K}{2 r}$

$$
\frac{d^{2} \psi}{d s^{2}}-\frac{2 E}{K^{2}} \psi=0
$$

Esta ecuación diferencial ordinaria de segundo orden tiene por solución

$$
\psi(s)=C \sin \sqrt{-\frac{2 E}{K^{2}}} s .
$$

Imponiendo que $\psi(0)=\psi(L)=0$, por tanto debe cumplirse que

$$
\sqrt{-\frac{2 E}{K^{2}}} L=n \pi \text { ó sabiendo que } \quad L=\sqrt{-\frac{K^{2}}{2 E}} 2 \pi
$$

se obtiene

$$
\sqrt{-\frac{2 E}{K^{2}}} \sqrt{-\frac{K^{2}}{2 E}} 2 \pi=n \pi \Rightarrow n=n_{f}=2
$$

Ell nivel de energía más estable o nivel fundamental será $E_{2}=E_{f}$, cuyo valor es

$$
E_{f}=-\frac{Z^{2} e^{4}}{\left(4 \pi \epsilon_{0}\right)^{2} 2 \hbar^{2}}
$$

Supongamos que tenemos un átomo hidrogenoide excitado en un estado inicial de energía $E_{i}$

$$
E_{i}=-\frac{Z^{2} e^{4}}{\left(4 \pi \epsilon_{0}\right)^{2} 2 n_{i}^{2} \hbar^{2}} .
$$

la diferencia de energías entre el estado inicial y el final será

$$
E_{i}-E_{f}=\frac{Z^{2}}{2}\left(\frac{1}{n_{f}^{2}}-\frac{1}{n_{i}^{2}}\right) \frac{e^{4}}{\left(4 \pi \epsilon_{0}\right)^{2} \hbar^{2}}
$$

si consideramos al estado final como el fundamental $\left(n_{f}=2\right)$ tendremos

$$
\nu=\frac{E_{i}-E_{f}}{h}=\frac{Z^{2}}{2}\left(\frac{1}{4}-\frac{1}{n_{i}^{2}}\right) \frac{e^{4}}{\left(4 \pi \epsilon_{0}\right)^{2} \hbar^{2} h}
$$

reescribiendo la expresión anterior en términos del radio de Bohr

$$
a_{0}=\frac{\epsilon_{0} h^{2}}{\pi m_{e} e^{2}},
$$


obtenemos

$$
\nu=\frac{Z^{2}}{2}\left(\frac{1}{4}-\frac{1}{n_{i}^{2}}\right) \frac{e^{4}}{4 \pi \epsilon_{0} a_{0} h}, \quad \text { con } \quad n_{i} \in \mathbb{Z}, n_{i} \geq 3
$$

que coincide con las frecuencias emitidas por el átomo de Hidrógeno $(Z=1)$ $\nu=\left(\frac{1}{4}-\frac{1}{n^{2}}\right) 3,29 \times 10^{15} \mathrm{~Hz}$ (John J. Balmer).

\section{A.3. Cuantización topológica: aplicación al os- cilador armónico}

Planteamos el problema de cuantización en dos dimensiones

$$
L=\frac{1}{2} m\left[(\dot{x})^{2}+(\dot{y})^{2}\right]+\frac{1}{2}\left[k_{1} x^{2}+k_{2} y^{2}\right] .
$$

Geometrizamos el problema definiendo la métrica Jacobi como

$$
g_{i j}=2(E-V(x, y))\left(\begin{array}{ll}
1 & 0 \\
0 & 1
\end{array}\right)=2\left[E-\frac{1}{2}\left[k_{1} x^{2}+k_{2} y^{2}\right]\right]\left(\begin{array}{ll}
1 & 0 \\
0 & 1
\end{array}\right) .
$$

A continuación, calculamos la curvatura escalar, obteniendo

$$
\mathcal{R}=\frac{2\left(2 E\left(k_{1}+k_{2}\right)+k_{1}^{2} x^{2}+k_{2}^{2}+y^{2}-k_{1} k_{2}\left(x^{2}+y^{2}\right)\right)}{\left(2 E-k_{1} x^{2}-k_{2} y^{2}\right)^{3}} .
$$

Haciendo ahora $k_{2}=0$, tenemos

$$
\mathcal{R}=\frac{2 k_{1}\left(2 E+k_{1} x^{2}\right)}{\left(2 E-k_{1} x^{2}\right)^{3}} .
$$

El Teorema de Gauss-Bonnet/Cuantización

$$
\int_{R} \frac{\mathcal{R}}{2} d \sigma=2 \pi n, \quad \text { con } \quad n \in \mathbb{Z} \quad \text { y } d \sigma=|g| d x d y=\left(2 E-k_{1} x^{2}\right) d x d y .
$$

el intervalo de integración para $x$ será $\left[-x_{0}, x_{0}\right]$. Así’i

$$
\begin{gathered}
\pi \int_{-x_{0}}^{x_{0}} \frac{k_{1}\left(2 E+k_{1} x^{2}\right)}{\left(2 E-k_{1} x^{2}\right)^{2}} d x=2 \pi n \Rightarrow \\
\frac{k_{1} x_{0}}{k_{1} x_{0}^{2}-2 E}=n, \quad n \in \mathbb{Z} .
\end{gathered}
$$


Haciendo los cambios (referencia al artículo)

$$
x_{0}=\frac{1}{C}-\sqrt{\frac{1}{C^{2}}+\frac{2 E}{k}}, C=2\left(\frac{E}{\hbar \omega}-\frac{1}{2}\right) \quad \text { y } \quad \omega=\sqrt{\frac{k}{m}},
$$

se obtiene

$$
\left(-\frac{1}{2}+\frac{E}{\hbar \omega}\right)=n \Rightarrow E_{n}=\hbar \omega\left(n+\frac{1}{2}\right) .
$$

\section{A.4. Ecuación de Schrödinger y métrica Jacobi}

Tomemos la función de onda de una partícula como

$$
\psi(x, t)=e^{i(k x-\omega t)} .
$$

La función ( A.43) debe ser solución de la ecuación de estado de la partícula. Por tanto, es fácil comprobar que podemos definir los siguientes operadores:

operador momento:

$$
\hat{p}=-i \hbar \frac{\partial}{\partial x}
$$

operador energía:

$$
\hat{E}=i \hbar \frac{\partial}{\partial t}
$$

Sustituimos estos operadores en la expresión clásica para la energía $\hat{E}=\frac{\hat{p}^{2}}{2 m}+$ $\hat{V}$, obteniendo la Ecuación de Schrödinger

$$
-\frac{\hbar^{2}}{2 m} \frac{\partial^{2} \psi(x, t)}{\partial x^{2}}+\hat{V}(x, t) \psi(x, t)=i \hbar \frac{\partial \psi(x, t)}{\partial t} .
$$

Llamamos

$$
\hat{H}=-\frac{\hbar^{2}}{2 m} \frac{\partial^{2}}{\partial x^{2}}+\hat{V} \equiv \quad \text { operador hamiltoniano }
$$

con la ecuación de valores propios del operador hamiltoniano,

$$
H \psi(x, t)=E \psi(x, t) .
$$

Si tenemos una partícula de masa $m$ con energía total $E$ en una caja unidimensional de dimensiones $0 \leq x \leq L$, la correspondiente ecuación de Schrodinger tendrá la forma

$$
\frac{d^{2} \psi(x)}{d x^{2}}=\frac{2 m}{\hbar^{2}}[\hat{V}(x)-E] \psi(x) .
$$


Si $V(x)=0$ tenemos

$$
\frac{d^{2} \psi(x)}{d x^{2}}+\frac{2 m E}{\hbar^{2}} \psi(x)=0 .
$$

La ecuación ( A.50) diferencial ordinaria de segundo orden tiene por solución

$$
\psi(x)=C \sin \sqrt{\frac{2 m E}{\hbar^{2}}} x
$$

y debe cumplirse la condición de contorno de Dirichlet, esto es, $\psi(0)=\psi(L)=$ 0. Por tanto,

$$
E_{n}=\frac{n^{2} \pi^{2} \hbar^{2}}{2 m L^{2}}
$$

resolviendo la ecuación A.48 para esta energía $E_{n}$ obtenemos

$$
\psi_{n}(x)=C \sin \sqrt{\frac{2 m E_{n}}{\hbar^{2}}} x=C \sin \left(\frac{n \pi x}{L}\right) .
$$

Interpretando $\psi_{n}^{2}(x) d x$ como la probabilidad de encontrar una partícula en el estado $n$ en un intervalo $d x$ centrado en $x$, entonces habrá de cumplirse

$$
C^{2} \int_{0}^{L} \sin \left(\frac{n \pi x}{L}\right)=1 \Rightarrow C=\sqrt{\frac{2}{L}}
$$

Así, las funciones de onda toman la forma

$$
\psi_{n}(x)=\sqrt{\frac{2}{L}} \sin \left(\frac{n \pi x}{L}\right)
$$

Esta cuantización presupone una partícula libre $V=0$ de masa $m$ sobre una geometría euclídea, es decir, de tensor métrico en coordenadas cartesianas de la forma $a_{i j}(\mathbf{q})=\delta_{i j}$.

Si en un espacio euclídeo las geodésicas son las rectas habituales, en una geometría elíptica, en particular esférica con la métrica Jacobi son circunferencias.

La dependencia encontrada anteriormente entre la longitud de la caja $L$ y la energía de la partícula $E$ (que, en principio parecen independientes), surge de una manera mucho más natural cuando se buscan las geodésicas análogas a las rectas euclídeas pero en la variedad mecánica, pues en ésta, la única geodésica de radio constante es $r=-\frac{K}{2 E}$.

La longitud de esta circunferencia sería

$$
L=\sqrt{\frac{-K^{2}}{2 E}} 2 \pi
$$


expresión que relaciona directamente la longitud $L$ con la energía de la ondapartícula E.

En el caso euclídeo, la geometría subyacente (con tensor métrico $\delta_{i j}$ ) y la energía $E$ de la partícula son independientes. Esto implica que se puede tener una experiencia física de cualquier escala de energías y los datos experimentales me indicarán que la geometría del espacio es euclídea (sabemos que a altas energías, entorno a velocidades próximas a $c$, se observa que la naturaleza del espacio no es euclídea sino pseudoeuclídea); en cambio en la variedad mecánica la energía está incluida ya dentro de la geometría espacio-temporal sobre la que se desarrolla el hecho físico.

En este último caso, la energía se invierte en curvar el espacio de configuración, de ahí que toda partícula en esta mecánica sea libre, esto es, le hemos quitado la energía a la partícula y se la hemos dado al espacio.

Así es, que no es necesario plantear la ecuación de Schrödinger para concluir la cuantización de la energía, ésta puede reproducirse si sustituimos la geometría euclídea subyacente por otra elíptica representada con la métrica de Jacobi $g_{i j}=2(E-V(\mathbf{q})) a_{i j}(\mathbf{q})$ de potencial central $V(r)$ y resolviendo la ecuación de Jacobi-Levi-Civita sobre una trayectoria periódica.

Esto es, debemos generar curvatura con alguna función potencial, ahora bien, sabemos por el Teorema de Bertrand que los únicos potenciales que generan órbitas cerradas estables tienen la forma

$$
V(r)=-K r^{-1} \text { y } V(r)=K r .
$$

Para reproducir el estudio de la ecuación de Shrödinger en el espacio euclídeo basta con curvar el espacio utilizando el potencial $V(r)=-K r^{-1}$ y estudiar la ecuación de JLC en dicha variedad mecánica.

Por simplicidad elegimos una circunferencia de radio $r=$ constante y aplicando la cuantización/conservación topológica podemos encontrar que $r=-\frac{K}{2 E}$ es la única trayectoria (geodésica) con $r=$ constante.

Así la curvatura escalar sobre esta curva será

$$
\frac{d^{2} \psi(s)}{d s^{2}}=-\frac{\mathcal{R}(s)}{2} \psi(s)=-\frac{2 E}{K^{2}} \psi(s)
$$

como se procedió en el caso anterior podemos determinar la cuantización de la energía como

$$
E_{n}=-\frac{n^{2} \pi^{2} K^{2}}{2 L^{2}}
$$


con $L \equiv$ longitud circunferencia con métrica Jacobi. Por tanto, resolviendo la ecuación de JLC para estos valores de energía tenemos

$$
\frac{d^{2} \psi_{n}}{d s^{2}}=\frac{2 E_{n}}{K^{2}} \psi_{n}=\frac{n^{2} \pi^{2}}{L^{2}} \psi_{n}
$$

con solución

$$
\psi_{n}(s)=C \sin \left(\frac{n \pi}{L} s\right) .
$$

Calculando el valor de $C$ realizado previamente obtenemos la expresión para las funciones de onda

$$
\psi_{n}(s)=\sqrt{\frac{2}{L}} \sin \left(\frac{n \pi s}{L}\right)
$$

con $s \equiv$ longitud de arco de la curva, coincidente con funciones de onda para ecuación de Schrödinger A.55). 


\section{Apéndice B}

\section{Geometría diferencial. Tensores}

Descartes inventa la Geometría analítica, esto es, una aritmetización de la Geometría, traduce a relaciones numéricas (literal, ecuación, solución, etc...) lo que eran relaciones geométricas (punto, recta, paralelismo, coincidencia, perpendicularidad,etc...) aritmetizando así la Geometría, las relaciones de extensión al dominio de los números.

Podríamos definir objeto geométrico como aquello que puede ser descrito sin hacer referencia a ningún sistema coordenado especial, esto es, aquello definible independientemente del sistema coordenado utilizado, sin una previa aritmetización, sin previa identificación numérica.

En primer lugar generalizamos el concepto de espacio a través de las variedades diferenciables.

El concepto de variedad generaliza la idea de superficie regular, estudia las propiedades geométricas sin la necesidad de que dicho objeto geométrico esté incluido en $\mathbb{R}^{3}$. Además, tendrá estructura diferenciable, esto es, la diferenciabilidad significa que podemos definir 1-formas y vectores, o dicho de otra forma, vectores covariantes y vectores contravariantes, respectivamente.

Además de tener puntos podemos hacer análisis, ya que permite definir o, más bien, servir de dominio de un campo escalar $\phi$ que permite además diferenciarlo en cualquier punto de este espacio.

Las variedades son localmente trozos de $\mathbb{R}^{n}$, pero globalmente pueden curvarse adoptando diferentes formas, entendiendo por forma una manera particular de ocupar el espacio.

La geometría diferencial a través del concepto de variedad diferenciable pretende independizarse del concepto de forma, en la medida de que la manera en que una superficie u objeto geométrico ocupa el espacio no describe nada fundamental de ella, sino más bien, es una representación particular de otras 
magnitudes geométricas intrínsecas y, por tanto, mucho más fundamentales que esta primera.

Toda representación mental como tal es una comparación.

Una Topología sobre un conjunto $M$ es una clase $\mathcal{T}$ de subconjuntos de $M$ que cumplen:

1.- La intersección de un número finito de conjuntos de $\mathcal{T}$ es otro conjunto en $\mathcal{T}$.

2.- La unión de cualquier número de conjuntos de $\mathcal{T}$ es otro conjunto en $\mathcal{T}$.

3.- El cunjunto vacío $\mathcal{O}$ y el total $M$ están en $\mathcal{T}$.

El par $(M, \mathcal{T})$ es un espacio topológico y los conjuntos de $\mathcal{T}$ se llaman conjuntos abiertos de este espacio.

Un espacio topológico se llama espacio Hausdorff si para todo par de puntos distintos $p \in M$ y $q \in M$, existen conjuntos abiertos $\mathcal{A}$ y $\mathcal{B}$ tal que $p \in \mathcal{A}$ y $q \in \mathcal{B}$ y $\mathcal{A} \cap \mathcal{B}=\emptyset$

Cualquier conjunto abierto de $\mathcal{T}$ que contenga a $p$ se llamará entorno abierto de $p$.

Sea $M$ un espacio topológico, y $U$ un entorno abierto del punto $p \in M$. Si existe un homeomorfismo $h: U \rightarrow \mathbb{R}^{n}$ sobre un conjunto abierto de $\mathbb{R}^{n}$, al par $(U, h)$ se le llama carta de dimension $\mathbf{n}$ con entorno coordenado $\mathrm{U}$.

Un atlas en $M$ es una colección de cartas $\left\{U_{\alpha}, h_{\alpha}\right\}$ tal que los conjuntos $U_{\alpha}$ constituyen un recubrimiento abierto de $M$.

Una variedad topológica es un espacio separable, Hausdorff que puede ser cubierto por un atlas.

Consideremos un par de cartas $\left(U_{1}, h_{1}\right)$ y $\left(U_{2}, h_{2}\right)$, tal que $W=U_{1} \cap U_{2} \neq \emptyset$, y consideremos el punto $p \in W$. Así, $h_{1}(p)=\left(u^{1}, u^{2}, \cdots, u^{n}\right)$ y $h_{2}(p)=$ $\left(\bar{u}^{1}, \bar{u}^{2}, \cdots, \bar{u}^{n}\right)$, lo que define el homeomorfismo

$$
\begin{gathered}
h_{1} \circ h_{2}^{-1}: \mathbb{R}^{n} \longrightarrow \mathbb{R}^{n}, \\
h_{1} \circ h_{2}^{-1}: h_{1}(W) \longrightarrow h_{2}(W) .
\end{gathered}
$$


Si las funciones $(\mathrm{B} .2)$ son de clase $C^{k}$, las cartas $\left(U_{1}, h_{1}\right)$ y $\left(U_{2}, h_{2}\right)$ se llaman $C^{k}$-compatibles.

Un $C^{\infty}$-atlas en $M$ es una colección de cartas $C^{\infty}-$ compatibles cuyos dominios cubren todo $\mathrm{M}$.

Un $C^{\infty}$-atlas se llama maximal si contiene todas las cartas $C^{\infty}$-compatibles con cualquiera de sus cartas se dice que este $C^{\infty}$-atlas define una estructura diferenciable en $\mathrm{M}$.

Una variedad $C^{\infty}$-diferenciable es una variedad topológica $M$ junto con una estructura diferenciable.

La dimensión de $M$ es la dimensión de sus cartas.

Con la estructura precedente de variedad diferenciable podemos definir el conjunto de todas las funciones $C^{\infty} U \longrightarrow \mathbb{R}$ definidas en un entorno $U$, de un punto $p \in M$, que denotaremos como $C_{p}^{\infty}$. Así, para cualquier función $f \in C_{p}^{\infty}$ la función $g=f \circ h^{-1}: \mathbb{R}^{n} \longrightarrow \mathbb{R}$ definimos

$$
\partial_{j} f=\frac{\partial g}{\partial u^{j}} \circ h=\frac{\partial f \circ h^{-1}}{\partial u^{j}} \circ h,
$$

es decir, $\partial_{j} f$ es la derivada respecto a $u^{j}$ de la función real $f \circ h^{-1}$ en el punto $h(p)$ de $\mathbb{R}^{n}$.

Ahora, sean $f, g \in C_{p}^{\infty}$ y $a, b \in \mathbb{R}$ se cumple,

$$
\begin{aligned}
\left.\partial_{j}(a f+b g)\right) & =a \partial_{j} f+b \partial_{j} g, \\
\partial_{j}(f g) & =f \partial_{j}(g)+g \partial_{j}(f) .
\end{aligned}
$$

Introducimos espacio tangente $T_{p}(M)$ en un punto $p$ de una variedad diferenciable $M$ como

El conjunto de todas las aplicaciones $X: C_{p}^{\infty} \longrightarrow \mathbb{R}$ que cumplen las siguientes condiciones para $f, g \in C_{p}^{\infty}$ y $a, b \in \mathbb{R}$

$$
\begin{aligned}
X(a f+b g)) & =a(X f)+b(X g), \\
X(f g) & =X(f) g(p)+f(p) X(g), \\
(X+Y) f & =X f+Y f \\
(a X) f & =a(X f) .
\end{aligned}
$$


Los elementos $X \in T_{p}(M)$ se llaman vectores tangentes de $M$ en $p$.

Este espacio vectorial de derivaciones existe porque se puede demostrar que los $n$ vectores coordenados $\partial_{j}$ previamente definidos (que existen, por la estructura diferenciable de la variedad) son una base del espacio tangente n-dimensional $T_{p}(M)$ en cada punto $p \in M$.

Por tanto $T_{p}(M)$ existe para cada $p \in M$.

En segunco lugar, generalizamos la idea de objeto geométrico:

Denominamos campo de un ente geométrico o simplemente ente geométrico de $P$-ésimo orden definido en un espacio $n$-dimensional (o en cierta región del mismo) a un sistema ordenado de funciones $\psi^{a}\left(x^{1}, x^{2}, \cdots, x^{n}\right)$ de las coordenadas de dicho espacio.

Tras cualquier transformación de sistemas de coordenadas $x^{\prime} i=\phi\left(x^{\prime 1}, x^{\prime 2}, \cdots, x^{\prime n}\right)$ varía según la ley

$$
\psi^{\prime a}\left(x^{\prime}\right)=Y^{a}\left(x^{\prime}, \frac{\partial x^{\prime i}}{\partial x^{k_{1}}}, \frac{\partial^{2} x^{\prime i}}{\partial x^{k_{1}} x^{k_{2}}}, \cdots, \frac{\partial^{p} x^{\prime i}}{\partial x^{k_{1}} \partial x^{k_{2}} \cdots \partial x^{k_{p}}}, \psi^{b}\left(x\left(x^{\prime}\right)\right)\right),
$$

$\operatorname{con}(a, b=1,2, \cdots, m)$

La clasificación de los entes geométricos depende del tipo de transformación (B.6).

Existen, por lo menos, dos entes geométricos de primer orden con un índice.

Un sistema de funciones que se transforma como una diferencial se denomina vector contravariante.

Un vector contravariante $a^{i}(x)$ al pasar a otro sistema de coordenadas se transforma según

$$
a^{\prime i}\left(x^{\prime}\right)=\frac{\partial x^{\prime i}}{\partial x^{k}} a^{k}\left(x\left(x^{\prime}\right)\right) .
$$

Un vector covariante $a_{i}(x)$ al pasar a otro sistema de coordenadas, se transforma según

$$
a_{i}^{\prime}\left(x^{\prime}\right)=\frac{\partial x^{k}}{\partial x^{\prime i}} a_{k}\left(x\left(x^{\prime}\right)\right)
$$

La generalización del vector (ente geométrico de primer orden) es el tensor. 
Llamaremos tensor $k$ veces covariante y $l$ veces contravariante $T_{i_{1} i_{2} \cdots i_{k}}^{j_{1} j_{2} \cdots j_{l}}(x)$ a un ente geométrico que al pasar a otro sistema de coordenadas se transforma según

$$
T_{i_{1} i_{2} \cdots i_{k}}^{j_{1} j_{2} \cdots j_{l}}\left(x^{\prime}\right)=\frac{\partial x^{a_{1}}}{\partial x^{\prime i_{1}}} \frac{\partial x^{a_{2}}}{\partial x^{\prime i_{2}}} \cdots \frac{\partial x^{a_{k}}}{\partial x^{\prime i_{k}}} \times \begin{aligned}
& \times \frac{\partial x^{\prime} j_{1}}{\partial x^{b_{1}}} \frac{\partial x^{\prime} j_{2}}{\partial x^{b_{2}}} \cdots \frac{\partial x^{\prime} j_{l}}{\partial x^{b_{l}}} T_{a_{1} a_{2} \cdots a_{k}}^{b_{1} b_{2} \cdots b_{l}}\left(x\left(x^{\prime}\right) \mathrm{B}\right.
\end{aligned}
$$

Un sistema de funciones que se transforma como un gradiente se denomina vector contravariante.

El campo escalar $\psi^{\prime}\left(x^{\prime}\right)=\psi\left(x\left(x^{\prime}\right)\right)$ sería el caso particular para $k=0, l=1$

(B.7) sería el caso particular para $k=0, l=1$

(B.8) sería el caso particular para $k=1, l=0$

Aclaremos algo más el concepto de ente geométrico y de tensor utilizando un lenguaje más cordial.

En principio llamamos vector a un segmento orientado uniendo dos puntos (origen y extremo), y llamaremos componentes al conjunto de los números que se obtienen como las diferencias coordenadas entre el punto origen y el extremo.

Convenio de sumación de Einstein: Cada vez que en una expresión indicial aparezca un índice somo subíndice y el mismo como superíndice se debe sobreentender que se realiza una suma de ambos índices con todos sus posibles valores, así

$$
\begin{aligned}
& A_{\alpha} B^{\alpha}=\sum_{\alpha=1}^{N} A_{\alpha} B^{\alpha}=A_{1} B^{1}+A_{2} B^{2}+\ldots+A_{N} B^{N}, \\
& A_{\alpha \beta} B^{\gamma \beta \nu}=A_{\alpha 1} B^{\gamma 1 \nu}+A_{\alpha 2} B^{\gamma 2 \nu}+\ldots+A_{\alpha N} B^{\gamma N \nu} .
\end{aligned}
$$

En un cierto sistema de coordenado $O$, el vector se expresará como $\mathbf{V}=V^{\alpha} \mathbf{e}_{(\alpha)}$.

Si expresamos este mismo vector en otro sistema coordenado $\bar{O}$ como $\mathbf{V}=$ $V^{\bar{\alpha}} \mathbf{e}_{(\bar{\alpha})}$

Esto se denomina transformación pasiva, ya que, cambiamos las coordenadas, pero no el vector.

Las coordenadas del vector se han transformado como

$$
V^{\bar{\alpha}}=\Lambda_{\beta}^{\bar{\alpha}} V^{\beta}
$$


$\operatorname{con} \Lambda_{\beta}^{\bar{\alpha}}=$ la matriz de transformación.

Si abstraemos la idea previa de vector, entonces podemos redefinir a éste como una colección de números (sus componentes en el sistema coordenado $O$ ) $V^{\alpha}$ junto con una regla de cambio de otro sistema coordenado, digamos $O$ definida por

$$
V^{\bar{\alpha}}=\Lambda_{\beta}^{\bar{\alpha}} V^{\beta}
$$

\section{B.1. Álgebra vectorial}

Podemos definir un operación interna $(+)$ llamada Suma de vectores y una operación externa (.) con elementos de un cuerpo $(\mathbb{R})$ llamada Producto de escalar por vector que dota al conjunto de vectores de estructura de espacio vectorial.

En cualquier sistema coordenado podemos definir unos vectores especiales, llamados, base del espacio vectorial $\mathbf{e}_{(\alpha)}$ donde

$$
\left(\mathbf{e}_{(\alpha)}\right)^{\beta}=\delta_{\alpha}^{\beta}=\left\{\begin{array}{ccc}
1 & \text { si } & \alpha=\beta \\
0 & \text { si } & \alpha \neq \beta
\end{array}\right.
$$

Cualquier vector puede ser expresado como combinación lineal de estos elementos de la base.

$$
\boldsymbol{V}=V^{\alpha} \mathbf{e}_{(\alpha)}
$$

\section{Transformación de los vectores de la base}

Supongamos que tenemos otro sistema coordenado con diferente base $\left\{\mathbf{e}_{(\bar{\alpha})}\right\}$ cualquier vector, por ser un ente geométrico, cumplirá

$$
V^{\alpha} \mathbf{e}_{(\alpha)}=V^{\bar{\alpha}} \mathbf{e}_{(\bar{\alpha})}=\underbrace{\Lambda_{\beta}^{\bar{\alpha}}}_{(\overline{B, 13}} V^{\beta} \mathbf{e}_{(\bar{\alpha})} .
$$

Cambiando $\alpha \leftrightarrow \beta$, obtenemos la igualdad

$$
V^{\alpha} \mathbf{e}_{(\alpha)}=V^{\alpha} \Lambda_{\alpha}^{\bar{\beta}} \mathbf{e}_{(\bar{\beta})} .
$$

Como la igualdad ( $\mathrm{B} .16)$ debe ser válida para cualquier vector $\boldsymbol{V}$ ha de serlo para cualquier conjunto de valores de $\left\{V^{\alpha}\right\}$. Por ello, se cumple que los elementos de la base se transforman de la siguiente manera

$$
\mathbf{e}_{(\alpha)}=\Lambda_{\alpha}^{\bar{\beta}} \mathbf{e}_{(\bar{\beta})}
$$


Comparando (B.13) y (B.18) vemos que, cuando hacemos un cambio de coordenadas, los vectores de la base y las componentes de los vectores cambian de manera diferente, esto es,

$$
\begin{gathered}
V^{\bar{\alpha}}=\Lambda_{\beta}^{\bar{\alpha}} V^{\beta} \quad \text { Cambio de componentes de vector } \boldsymbol{V} \text { de sist. coord. } O \text { a } \bar{O}, \\
V^{\alpha}=\Lambda_{\bar{\beta}}^{\alpha} V^{\bar{\beta}} \quad \text { Cambio de componentes de vector } \boldsymbol{V} \text { de sist. coord. } \bar{O} \text { a } O, \\
\mathbf{e}_{(\alpha)}=\Lambda_{\alpha}^{\bar{\nu}} \mathbf{e}_{(\bar{\nu})} \quad \text { Cambio de vectores de la base de sist. coord. } O \text { a } \bar{O}, \\
\mathbf{e}_{(\bar{\alpha})}=\Lambda_{\bar{\alpha}}^{\nu} \mathbf{e}_{(\nu)} \quad \text { Cambio de vectores de la base de sist. coord. } \bar{O} \text { a } O .
\end{gathered}
$$

Teniendo en cuenta que $\Lambda_{\alpha}^{\beta} \Lambda_{\beta}^{\nu}=\delta_{\alpha}^{\nu}$, es decir, que $\Lambda_{\alpha}^{\beta}$ y $\Lambda_{\beta}^{\nu}$ son matrices inversas y $\delta_{\alpha}^{\nu}$ la matriz identidad entonces

$$
\boldsymbol{V}=V^{\alpha} \mathbf{e}_{(\alpha)}=\underbrace{\Lambda_{\bar{\beta}}^{\alpha} V^{\bar{\beta}}}_{V^{\alpha}} \underbrace{\Lambda_{\alpha}^{\bar{\nu}} \mathbf{e}_{(\bar{\nu})}}_{\mathbf{e}_{(\alpha)}}=\underbrace{\Lambda_{\bar{\beta}}^{\alpha} \Lambda_{\alpha}^{\bar{\nu}}}_{\delta_{\overline{\bar{\beta}}}^{\bar{\nu}}} V^{\bar{\beta}} \mathbf{e}_{(\bar{\nu})}=V^{\bar{\nu}} \mathbf{e}_{(\bar{\nu})}=\boldsymbol{V}
$$

\section{Tensores}

Un tensor de tipo $\left(\begin{array}{c}0 \\ N\end{array}\right)$ es una función, lineal en cada uno de sus $N$ argumentos, que asigna a $N$ vectores un número real.

$$
T: \mathbb{V} \times \mathbb{V} \times \ldots \mathbb{V} \longrightarrow \mathbb{R}
$$

Importante: Un tensor debe devolver el mismo número real independientemente del sistema coordenado utilizado para expresar los vectores.

\section{Componentes de un tensor}

Las componentes de un tensor de tipo $\left(\begin{array}{c}0 \\ N\end{array}\right)$ en un determinado sistema coordenado $\mathbb{O}$, son los valores obtenidos cuando en los argumentos del tensor sustituimos los elementos de una base $\left\{\mathbf{e}_{\alpha}\right\}$ de $\mathbb{O}$.

\section{Tensores de tipo $\left(\begin{array}{l}0 \\ 1\end{array}\right)$}

Estos tensores también son llamados 1-formas, covectores o vectores covariantes.

$$
\tilde{w}: \mathbb{V} \longrightarrow \mathbb{R}
$$

El espacio de 1-formas se denomina también espacio vectorial dual $\mathbb{V}^{*}$.

Las componentes de las 1-formas son 


$$
w_{\alpha}=w\left(\mathbf{e}_{\alpha}\right)
$$

Convenio: componentes escritas con subíndices serán componente de una 1forma, en cambio, si están escritas con superíndices lo serán pero de un vector.

Así

$$
\tilde{w}(\boldsymbol{V})=\tilde{w}\left(V^{\alpha} \mathbf{e}_{\alpha}\right)=V^{\alpha} \tilde{w}\left(\mathbf{e}_{\alpha}\right)=V^{\alpha} w_{\alpha}
$$

Esta operación se denomina contracción.

Las componentes de $\tilde{w}$ en otra base $\left\{\mathbf{e}_{\bar{\alpha}}\right\}$ será

$$
\begin{gathered}
w_{\bar{\alpha}}=\tilde{w}\left(\mathbf{e}_{\bar{\alpha}}\right)=\tilde{w}\left(\Lambda_{\bar{\beta}}^{\alpha} \mathbf{e}_{\bar{\alpha}}\right)=\Lambda_{\bar{\beta}}^{\alpha} \bar{w}\left(\mathbf{e}_{\alpha}\right)=\Lambda_{\bar{\beta}}^{\alpha} w_{\alpha} \\
w_{\bar{\alpha}}=\Lambda_{\bar{\beta}}^{\alpha} w_{\alpha}
\end{gathered}
$$

luego las componentes de una 1-forma se transforman igual que los vectores de la base e inversamente a las componentes de un vector.

$$
V^{\bar{\alpha}} w_{\bar{\alpha}}=\Lambda_{\beta}^{\bar{\alpha}} V^{\beta} \Lambda_{\bar{\alpha}}^{\nu} w_{\nu}=\Lambda_{\beta}^{\bar{\alpha}} \Lambda_{\bar{\alpha}}^{\nu} V^{\beta} w_{\nu}=\delta_{\beta}^{\nu} V^{\beta} w_{\nu}=V^{\beta} w_{\beta}
$$

Por tanto la expresión $V^{\alpha} w_{\alpha}$ es invariante, esto es, no depende del sistema coordenado utilizado.

Ahora se justifican los términos covariante y contravariante:

covariante: Se transforma de la misma forma que lo hacen los vectores de la base.

contravariante : Se transforma de forma inversa a como lo hacen los vectores de la base.

Bases de las 1-formas

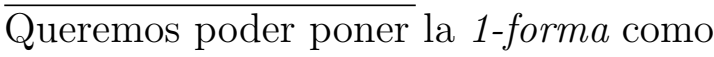

$$
\tilde{w}=w_{\alpha} \tilde{\mathbf{e}}^{(\alpha)}
$$

debe cumplirse

$$
\tilde{w}(\boldsymbol{V})=w_{\alpha} \tilde{\mathbf{e}}^{(\alpha)}(\boldsymbol{V})=w_{\alpha} \mathbf{e}^{\alpha}\left(V^{\nu} \mathbf{e}_{\nu}\right)=w_{\alpha} V^{\nu} \mathbf{e}^{\alpha}\left(\mathbf{e}_{\nu}\right)=w_{\alpha} V^{\alpha}
$$

por tanto

$$
\tilde{\mathbf{e}}^{\alpha}\left(\mathbf{e}_{(\beta)}\right)=\delta_{\beta}
$$

Observaciones:

1.-La definición de la base $\left\{\tilde{\mathbf{e}}^{\alpha}\right\}$ es en términos de $\left\{\tilde{\mathbf{e}}_{\alpha}\right\}$, esto es, una vez fijada la base $\left\{\mathbf{e}_{\alpha}\right\}$ del espacio vectorial $\mathbb{V}$ queda definida, de forma natural, una base 
$\left\{\tilde{\mathbf{e}}^{\alpha}\right\}$ en el espacio dual $\mathbb{V}^{*}$.

En otras palabras, cada base $\left\{\mathbf{e}_{\alpha}\right\}$ tiene su propia base dual $\left\{\tilde{\mathbf{e}}^{\alpha}\right\}$.

2.- Vectores y 1-formas aunque representados en sus respectivas bases por $N$ valores, tienen un significado geométrico diferente.

Se puede demostrar que ante un cambio de base $\left\{\mathbf{e}_{\alpha}\right\} \longrightarrow\left\{\mathbf{e}_{\bar{\alpha}}\right\}$, Los elementos de la base dual $\left\{\tilde{\mathbf{e}}^{\alpha}\right\}$ cambian como

$$
\tilde{\mathbf{e}}^{\bar{\alpha}}=\Lambda_{\beta}^{\bar{\alpha}} \tilde{e}^{\beta}
$$

Se observa que se transforman como las componentes de un vector, e inversamente a las componentes de una 1-forma.

$\underline{\text { Imagen geométrica de una 1-forma }}$

De igual manera que un vector suele imaginarse como un segmento orientado, una 1-forma la imaginaremos como un conjunto de superficies.

La magnitud de la 1-forma tendrá que ver con el espaciado de estas superficies, de tal manera que, cuanto mayor sea la magnitud de la 1-forma menor será el espaciado entre las diferentes superficies.

Así pues, cuando la 1-forma se contrae con un vector, en ocasiones se dice que el tensor (en este caso la 1-form) se satura con un vector, el número resultante de esta operación puede interpretarse como el número de superficies atravesadas (pinchadas) por dicho vector.

Puede observarse que en este conjunto de superficies, podemos considerar un vector especial entre todos los que pueden atravesar dichas superficies y es aquel que atraviesa más superficies por unidad de longitud, este vector será el vector gradiente, lo que motiva asociar a una 1-forma un único vector, esta correspondencia unívoca la veremos más adelante.

\section{$\underline{\text { Ejemplo }}$}

Podemos escribir el potencial gravitatorio en un punto del espacio $\left\{x^{\alpha}\right\}$ como $V\left(x^{\alpha}\right)$

El gradiente de $V$ es un 1-forma, esto es, $\left\{\frac{\partial V\left(x^{\alpha}\right)}{\partial x^{\alpha}}\right\}$ representan las componentes de una 1-forma.

Por tanto podré expresar el gradiente como combinación lineal de elemen- 
tos de la base dual $\tilde{\mathbf{e}}^{\alpha}$ como $d V=\frac{\partial V\left(x^{\alpha}\right)}{\partial x^{\alpha}} \tilde{\mathbf{e}}^{\alpha}$

¿ Cómo puedo entender $d V$ como una 1 -forma $\tilde{d} V: \mathbb{V} \longrightarrow \mathbb{R}$ ?

Tomando un vector $\boldsymbol{A}$ en un punto

$$
\tilde{d V}(\boldsymbol{A})=\frac{\partial V(\boldsymbol{x})}{\partial x^{\alpha}} \tilde{\mathbf{e}}^{\alpha}\left(A^{\beta} \mathbf{e}_{\beta}\right)=\frac{\partial V(\boldsymbol{x})}{\partial x^{\alpha}} A^{\beta} \delta_{\beta}^{\alpha}=\frac{\partial V(\boldsymbol{x})}{\partial x^{\alpha}} A^{\alpha}
$$

Si entendemos que el vector $\boldsymbol{A}$ representa el vector tangente a una cuva $\gamma(\tau)$ que en el sistema coordenado se escribe $\dot{\gamma}(\tau)=\frac{\partial x^{\alpha}}{\partial \tau} \mathbf{e}_{\alpha}$ en un punto $P$, entonces

$$
A^{\alpha}=\frac{\partial x^{\alpha}}{\partial \tau}
$$

entonces

$$
\tilde{d V}(\boldsymbol{A})=\frac{\partial V(\boldsymbol{x})}{\partial x^{\alpha}} \tilde{\mathbf{e}}^{\alpha}\left(\frac{\partial x^{\alpha}}{\partial \tau} \mathbf{e}_{\alpha}\right)=\frac{\partial V(\boldsymbol{x})}{\partial x^{\alpha}} \frac{\partial x^{\alpha}}{\partial \tau}=\frac{d V(\boldsymbol{x}(\tau))}{d \tau}
$$

que representa la variación del campo $V(\boldsymbol{x})$ a lo largo de la curva $\gamma(\tau)$ en el punto $P$.

Así

$$
\begin{aligned}
\tilde{d} V: \mathbb{V} & \longrightarrow \mathbb{R} \\
A^{\alpha}=\frac{\partial x^{\alpha}}{d \tau} & \longrightarrow \frac{d V(\boldsymbol{x})}{d \tau} \in \mathbb{R}
\end{aligned}
$$

donde $\frac{d V(\boldsymbol{x})}{d \tau}$ representa la variación del escalar $V(\boldsymbol{x})$ en la dirección del vector tangente a la curva $\gamma(\tau)$ en el punto $P$.

\section{1-formas normales}

Una 1-forma es normal a una superficie si su valor sobre todo vector del espacio tangente a la superficie es cero.

Esta definición no necesita del concepto de perpendicularidad, por tanto, no necesita ninguna estructura métrica.

Tensores de tipo $\left(\begin{array}{l}0 \\ 2\end{array}\right)$

Son aquellos tensores que para cada pareja de vectores devuelven un número real. 


$$
\mathbf{T}: \mathbb{V} \times \mathbb{V} \longrightarrow \mathbb{R}
$$

Componentes

$$
T_{\alpha \beta}=\mathbf{T}\left(\mathbf{e}_{\alpha}, \mathbf{e}_{\beta}\right)
$$

Para vectores arbitrarios $\boldsymbol{A}=A^{\alpha} \mathbf{e}_{\alpha}$ y $\boldsymbol{A}=A^{\beta} \mathbf{e}_{\beta}$ tenemos

$$
\mathbf{T}\left(A^{\alpha} \mathbf{e}_{\alpha}, A^{\beta} \mathbf{e}_{\beta}\right)=A^{\alpha} B^{\beta} \mathbf{T}\left(\mathbf{e}_{\alpha}, \mathbf{e}_{\beta}\right)=A^{\alpha} B^{\beta} T_{\alpha \beta}
$$

Qué base $\left\{\tilde{\mathbf{e}}^{\alpha \beta}\right\}$ tendrán estos tensores?

$$
\begin{gathered}
\mathbf{T}=T_{\alpha \beta} \tilde{\mathbf{e}}^{\alpha \beta} \\
T_{\mu \nu}=\mathbf{T}\left(\mathbf{e}_{\mu}, \mathbf{e}_{\nu}\right)=T_{\alpha \beta} \tilde{\mathbf{e}}^{\alpha \beta}\left(\mathbf{e}_{\mu}, \mathbf{e}_{\nu}\right) \Rightarrow \tilde{\mathbf{e}}^{\alpha \beta}\left(\mathbf{e}_{\mu}, \mathbf{e}_{\nu}\right)=\delta^{\alpha}{ }_{\mu} \delta^{\beta}{ }_{\nu}
\end{gathered}
$$

como vimos anteriormente $\tilde{\mathbf{e}}^{\alpha}\left(\mathbf{e}_{\mu}\right)=\delta^{\alpha}{ }_{\mu}$

por tanto

$$
\tilde{\mathbf{e}}^{\alpha \beta}\left(\mathbf{e}_{\mu}, \mathbf{e}_{\nu}\right)=\delta^{\alpha}{ }_{\mu} \delta^{\beta}{ }_{\nu}=\tilde{\mathbf{e}}^{\alpha}\left(\mathbf{e}_{\mu}\right) \tilde{\mathbf{e}}^{\beta}\left(\mathbf{e}_{\nu}\right)
$$

por tanto $\tilde{\mathbf{e}}^{\alpha \beta}=\tilde{\mathbf{e}}^{\alpha} \otimes \tilde{\mathbf{e}}^{\beta}$ con $\otimes \equiv$ producto tensorial, definido como $\left(\tilde{\mathbf{v}}^{\alpha} \otimes\right.$ $\left.\tilde{\mathbf{w}}^{\beta}\right)\left(\mathbf{e}_{\mu}, \mathbf{e}_{\nu}\right)=\tilde{\mathbf{v}}^{\alpha}\left(\mathbf{e}_{\mu}\right) \tilde{\mathbf{w}}^{\beta}\left(\mathbf{e}_{\nu}\right)$

Por tanto, $\tilde{\mathbf{e}}^{\alpha \beta}=\tilde{\mathbf{e}}^{a l p h a} \otimes \tilde{\mathbf{e}}^{b e t a}$ son los elementos de la base así podemos expresar cualquier tensor $\left(\begin{array}{l}0 \\ 2\end{array}\right)$ como

$$
\mathbf{T}=T_{\alpha \beta} \tilde{\mathbf{e}}^{\alpha} \otimes \tilde{\mathbf{e}}^{\beta}
$$

\section{Simetrías}

Diremos que un tensor $\mathbf{T}$ es simétrico si cumple

$$
\mathbf{T}(\boldsymbol{A}, \boldsymbol{B})=\mathbf{T}(\boldsymbol{B}, \boldsymbol{A}), \text { en coordenadas, } T_{\alpha \beta}=T_{\beta \alpha}
$$

La parte simétrica de un tensor se denota por $T_{(\alpha \beta)}$ y se define como

$$
T_{(\alpha \beta)}=\frac{1}{2}\left(T_{\alpha \beta}+T_{\beta \alpha}\right)
$$

Diremos que un tensor $\mathbf{T}$ es antisimétrico si cumple

$$
\mathbf{T}(\boldsymbol{A}, \boldsymbol{B})=-\mathbf{T}(\boldsymbol{B}, \boldsymbol{A}) \text {, en coordenadas, } T_{\alpha \beta}=-T_{\beta \alpha}
$$


La parte antisimétrica de un tensor se denota por $T_{[\alpha \beta]}$ y se define como

$$
T_{[\alpha \beta]}=\frac{1}{2}\left(T_{\alpha \beta}-T_{\beta \alpha}\right)
$$

Podemos, por tanto, descomponer todo tensor de forma única en su parte simétrica y en su parte antisimétrica de la forma

$$
T_{\alpha \beta}=T_{(\alpha \beta)}+T_{[\alpha \beta]}
$$

\section{$\underline{\text { Tensor métrico }}$}

Decimos que una variedad tiene estructura métrica si tiene definido en todos sus puntos un tensor $\left(\begin{array}{l}0 \\ 2\end{array}\right)$ simétrico (puede ser o no definido positivo).

Cuando hay definida una métrica en el espacio, desaparece la diferencia entre los previamente llamados covariante y contravariante, o equivalentemente entre covectores y vectores ya que podremos hacer la siguiente identificación via métrica

$$
\begin{aligned}
& \mathbf{g}(\boldsymbol{V}, \cdot) \equiv \tilde{V}(\cdot) \\
& g: \mathbb{V} \longrightarrow \mathbb{V}^{*} \\
& \boldsymbol{V} \longrightarrow \mathbf{g}(\boldsymbol{V}, \cdot)
\end{aligned}
$$

Sea el vector $\boldsymbol{V}=V^{\nu} \mathbf{e}_{\nu}$, ¿Qué componentes tendrá $\tilde{V}$ ?

$$
V_{\alpha}=V\left(\mathbf{e}_{\alpha}\right)=\mathbf{g}\left(\boldsymbol{V}, \mathbf{e}_{\alpha}\right)=\mathbf{g}\left(V^{\nu} \mathbf{e}_{\nu}, \mathbf{e}_{\alpha}\right)=V^{\nu} \mathbf{g}\left(\mathbf{e}_{\nu}, \mathbf{e}_{\alpha}\right)=V^{\nu} g_{\nu \alpha}
$$

por tanto las componentes de $\tilde{V}$ serán

$$
V_{\alpha}=V^{\nu} g_{\nu \alpha}
$$

Sea el covector $\tilde{V}=V_{\nu} \mathbf{e}^{\nu}$, ¿Qué componentes tendrá $\boldsymbol{V}$ ?

$$
V_{\alpha}=g_{\nu \alpha} V^{\nu}
$$

multiplicando ambos miembros de la igualdad por $g^{\nu \alpha}$, esto es, la matriz inversa de $g_{\nu \alpha}$ obtenemos

$$
g^{\beta \alpha} V_{\alpha}=g^{\beta \alpha} g_{\nu \alpha} V^{\nu}=\delta^{\beta}{ }_{\nu} V^{\nu}=V^{\beta}
$$

por tanto las componentes de $\boldsymbol{V}$ serán

$$
V^{\alpha}=V_{\nu} g^{\nu \alpha}
$$


Por tanto, en primer lugar, sabemos diferenciar las coordenadas de un vector de las de un covector sin más que observar si sus índices están arriba o abajo. En segundo lugar, si tenemos un espacio métrico podemos realizar la identificación entre vectores y covectores.

Si la anterior identificación existe, entonces ¿podemos hablar de la longitud/magnitud de una 1-forma?

La respuesta es afirmativa, ya que la 1-forma $\tilde{v}$ por definición debe tener la misma magnitud que su vector asociado $\boldsymbol{v}$, así

$$
\tilde{V}^{2}=g_{\alpha \beta} V^{\alpha} V^{\beta}
$$

de esta forma

$$
\tilde{V}^{2}=g_{\alpha \beta}\left(g^{\alpha \nu} V_{\nu}\right)\left(g^{\beta \mu} V_{\mu}\right)
$$

dado que $g_{\alpha \beta} g^{\alpha \nu}=\delta_{\beta}^{\nu}$ entonces

$$
\tilde{V}^{2}=g^{\mu \nu} V_{\nu} V_{\mu}
$$

Podemos definir un producto escalar entre 1-formas?

Efectivamente definimos dicho producto escalar como

$$
\tilde{V} \cdot \tilde{W}=\frac{1}{2}\left[\left(\tilde{V}^{2}+\tilde{W}^{2}\right)^{2}-\tilde{V}^{2}-\tilde{W}^{2}\right]
$$

Tensores de tipo $\left(\begin{array}{c}M \\ 0\end{array}\right)$

Serán funciones lineales de $M$ 1-formas en los números reales.

$$
T: \mathbb{V}^{*} \times \mathbb{V}^{*} \longrightarrow \mathbb{R}
$$

Una base de este tipo de tensores es $\mathbf{e}_{\alpha} \otimes \mathbf{e}_{\beta}$, así

$$
\begin{aligned}
& \quad T^{\alpha \beta}=\mathbf{T}\left(\tilde{\mathbf{e}}^{\alpha}, \tilde{\mathbf{e}}^{\beta}\right)=T^{\mu \nu}\left(\mathbf{e}_{\mu} \otimes \mathbf{e}_{\nu}\right)\left(\tilde{\mathbf{e}}^{\alpha}, \tilde{\mathbf{e}}^{\beta}\right)=T^{\mu \nu} \mathbf{e}_{\mu}\left(\tilde{\mathbf{e}}^{\alpha}\right) \cdot \mathbf{e}_{\nu}\left(\tilde{\mathbf{e}}^{\beta}\right)=T^{\mu \nu} \delta_{\mu}{ }^{\alpha} \delta_{\nu}{ }^{\beta} \\
& \text { siendo } \mathbf{e}_{\mu}\left(\tilde{\mathbf{e}}^{\alpha}\right)=\tilde{\mathbf{e}}^{\mu}\left(\mathbf{e}_{\alpha}\right)
\end{aligned}
$$

$$
\boldsymbol{V}(\tilde{p})=\tilde{p}(\boldsymbol{V})=p_{\text {alpha }} V^{\alpha}
$$

Tensores de tipo $\left(\begin{array}{c}M \\ N\end{array}\right)$ 
Serán funciones lineales de $M$ 1-formas y $N$ vectores en los números reales.

$$
T: \mathbb{V}^{*} \times \mathbb{V}^{*} \cdots \mathbb{V}^{*} \times \mathbb{V} \times \mathbb{V} \cdots \mathbb{V} \longrightarrow \mathbb{R}
$$

Una base para este espacio será

$$
\left\{\mathbf{e}_{\alpha} \otimes \mathbf{e}_{\beta} \otimes \cdots \mathbf{e}_{\gamma} \otimes \tilde{\mathbf{e}}^{\mu} \otimes \tilde{\mathbf{e}}^{\nu} \otimes \cdots \mathbf{e}^{\lambda}\right\}
$$

Un ejemplo de este tipo de tensores es la curvatura, que en un punto mide cuánto difiere la variedad Riemanniana de ser Euclidea.

Especial mención merece el tensor de curvatura de Riemann-Christoffel $R_{k l m}^{i}$ donde

$$
R_{i k l m}=\frac{1}{2}\left(\frac{\partial^{2} g_{m i}}{\partial x^{l} \partial x^{k}}+\frac{\partial^{2} g_{k l}}{\partial x^{i} \partial x^{m}}-\frac{\partial^{2} g_{i l}}{\partial x^{k} \partial x^{m}}-\frac{\partial^{2} g_{k m}}{\partial x^{i} \partial x^{l}}\right)+g_{q p}\left(\Gamma_{k l}^{p} \Gamma_{m i}^{q}-\Gamma_{k m}^{p} \Gamma_{i l}^{q}\right)
$$

Se puede definir como la correspondencia que asocia cada par $\mathbf{X}, \mathbf{Y} \in \chi(M)$ una aplicación

$R(\mathbf{X}, \mathbf{Y}): \chi(M) \longrightarrow \chi(M)$ dada por

$$
R(\mathbf{X}, \mathbf{Y}) \mathbf{Z}=\nabla_{\mathbf{Y}} \nabla_{\mathbf{X}} \mathbf{Y}-\nabla_{\mathbf{X}} \nabla_{\mathbf{Y}} \mathbf{X}+\nabla_{[\mathbf{X}, \mathbf{Y}]} \mathbf{Z} \quad \mathbf{Z} \in \chi(M)(\mathrm{B} .65)
$$

donde $\nabla$ es la conexión de Riemann en $M$.

En particular si el tensor de curvatura del espacio de Riemann es cero $R_{k l m}^{i}=0$ entonces dicho espacio se denomina espacio de Riemann plano.

Este tensor cumple varias identidades importantes:

$\underline{\text { Identidad de Ricci }}$

La alternización respecto cualesquiera tres índices es nula

$$
R_{[i k l] m}=\frac{1}{3 !}\left(R_{i k l m}+R_{l i k m}+R_{k l i m}\right)=0
$$

Identidad de Bianchi-Pádov

$$
R_{k l m ; p}^{i}+R_{k p l ; m}^{i}+R_{k m p ; l}^{i}=0
$$

El tensor de curvatura B.64 tiene

$$
N=\frac{n^{2}\left(n^{2}-1\right)}{12}
$$


componentes independientes.

$$
R_{k l m}^{i}=\frac{\partial \Gamma_{k m}^{i}}{\partial x^{l}}-\frac{\partial \Gamma_{k l}^{i}}{\partial x^{m}}+\Gamma_{l s}^{i} \Gamma_{k m}^{s}-\Gamma_{m s}^{i} \Gamma_{k l}^{s}
$$

Contraemos la expresión B.69 respecto a $i$ y a $l$ y encontramos así el tensor de Ricci

$$
R_{k m}=\frac{\partial \Gamma_{k m}^{i}}{\partial x^{i}}-\frac{\partial \Gamma_{k i}^{i}}{\partial x^{m}}+\Gamma_{i s}^{i} \Gamma_{k m}^{s}-\Gamma_{m s}^{i} \Gamma_{k i}^{s}
$$

Subiendo uno de los índices y contrayendo los índices del tensor obtenido podemos definir la curvatura escalar como

$$
R=R_{i}^{i}=g^{i m} R_{i m}
$$

La curvatura seccional en el punto $P$ generaliza la curvatura Gaussiana en variedades diferenciables inmersas en $\mathcal{R}^{3}$ a variedades de cualquier dimensión sin la necesidad de la existencia de un espacio ambiente y se define como sigue:

Dado un punto $p \in M$ y un subespacio dos dimensional $\sigma \subset T_{p} M$, el número real

$$
K(\mathbf{X}, \mathbf{Y})=K(\sigma)=\frac{<R(\mathbf{X}, \mathbf{Y}) \mathbf{X}, \mathbf{Y}>}{|\mathbf{X} \times \mathbf{Y}|^{2}}
$$

donde $\{\mathbf{X}, \mathbf{Y}\}$ es cualquier base de $\sigma$ and $|\mathbf{X} \times \mathbf{Y}|^{2}=|\mathbf{X}|^{2}|\mathbf{Y}|^{2}-<\mathbf{X}, \mathbf{Y}>^{2}$

Tensor nulo: Es aquel que tiene todas sus componentes iguales a cero en algún sistema de coordenadas.

Importante: Si un tensor es igual a cero en un sistema de coordenadas, entonces también lo será en cualquier otro.

\section{Álgebra tensorial}

En principio tenemos cuatro operaciones invariantes que podemos efectuar con vectores:

Adición : se aplica a tensores que tienen los mismos índices covariantes y contravariantes.

Se suman algebraicamente las componentes correspondientes de los tensores en cada punto del espacio.

$$
A_{i j}{ }^{k}(x)=B_{i j}{ }^{k}(x)+C_{i j}{ }^{k}(x)
$$


Multiplicación : se puede aplicar a tensores con diferente número de índices covariantes y contravariantes

$$
C_{i j l}{ }^{k m}=A_{i j}{ }^{k}(x) \cdot B_{l}{ }^{m}(x)
$$

Contracción : solo aplicable a tensores que tienen índices covariantes y contravariantes

La contracción del tensor $A_{\alpha_{1}, \alpha_{2}, \cdots, \alpha_{q}, \cdots, \alpha_{k}}^{\beta_{1}, \beta_{2}, \cdots \beta_{q}, \cdots, \beta_{k}}$ respecto a los índices p-ésimo contravariante y q-ésimo covariante se realiza multiplicando dicho tensor por el símbolo de Kronecker ${ }_{\beta_{q}}^{\alpha_{q}}$.

La contracción convierte un tensor de tipo $\left(\begin{array}{c}M \\ N\end{array}\right)$ en otro de tipo $\left(\begin{array}{c}M-1 \\ N-1\end{array}\right)$ Por ejemplo, el tensor $\mathbf{T}$ de tipo $\left(\begin{array}{l}2 \\ 2\end{array}\right)$

$$
\mathbf{T}: \mathbb{V}^{*} \times \mathbb{V}^{*} \times \mathbb{V} \times \mathbb{V} \longrightarrow \mathbb{R}
$$

cuya expresión como combinación lineal de elementos de la base es

$$
\mathbf{T}=T_{k l}^{i j} \mathbf{e}_{i} \otimes \mathbf{e}_{j} \otimes \tilde{\mathbf{e}}^{k} \otimes \tilde{\mathbf{e}}^{l}
$$

puede ser contraído, por ejemplo, respecto al primer índice contravariante y el segundo índice covariante como

$$
\begin{aligned}
& T^{i j}{ }_{k l} \mathbf{e}_{i} \otimes \mathbf{e}_{j} \otimes \tilde{\mathbf{e}}^{k} \otimes \tilde{\mathbf{e}}^{l}\left(\tilde{\mathbf{e}}^{m}, \cdot, \cdot, \mathbf{e}_{m}\right)= \\
& T^{i j}{ }_{k l} \delta_{i}^{m} \delta_{m}^{l} \tilde{\mathbf{e}}^{j} \otimes \mathbf{e}_{k}=T_{k m}^{m j} \mathbf{e}_{j} \otimes \tilde{\mathbf{e}}^{k}=\left(T_{k 1}^{1 j}+T_{k 2}^{2 j}+\cdots+T_{k n}^{n j}\right) \mathbf{e}_{j} \otimes \tilde{\mathbf{e}}^{k}
\end{aligned}
$$

o, por ejemplo, respecto al segundo índice contravariante y el segundo índice covariante como

$$
\begin{aligned}
& T^{i j}{ }_{k l} \mathbf{e}_{i} \otimes \mathbf{e}_{j} \otimes \tilde{\mathbf{e}}^{k} \otimes \tilde{\mathbf{e}}^{l}\left(\cdot, \tilde{\mathbf{e}}^{m}, \cdot, \mathbf{e}_{m}\right)= \\
& T^{i j}{ }_{k l} \delta_{j}^{m} \delta_{m}^{l} \mathbf{e}_{i} \otimes \tilde{\mathbf{e}}^{k}=T_{k m}^{i m} \mathbf{e}_{i} \otimes \tilde{\mathbf{e}}^{k}=\left(T_{k 1}^{i 1}+T_{k 2}^{i 2}+\cdots+T_{k n}^{i n}\right) \mathbf{e}_{i} \otimes \tilde{\mathbf{e}}^{k}
\end{aligned}
$$

en ambos casos la operación de contracción nos devuelve un tensor de tipo $\left(\begin{array}{l}1 \\ 1\end{array}\right)$

En el caso particular de un tensor $\mathbf{T}$ de tipo $\left(\begin{array}{l}1 \\ 1\end{array}\right)$

la operación de contracción devuelve un escalar, esto es,

$$
\mathbf{T}: \mathbb{V}^{*} \times \mathbb{V} \longrightarrow \mathbb{R}
$$

cuya expresión como combinación lineal de elementos de la base es

$$
\mathbf{T}=T_{j}^{i} \mathbf{e}_{i} \otimes \tilde{\mathbf{e}}^{j}
$$


la contracción sería

$$
T_{k}^{i} \mathbf{e}_{i} \otimes \tilde{\mathbf{e}}^{k}\left(\tilde{\mathbf{e}}^{m}, \mathbf{e}_{m}\right)=T_{k}^{i} \delta_{i}^{m} \delta_{m}^{k}=T_{m}^{m}=T_{1}^{1}+T_{2}^{2}+\cdots+T_{n}^{n} \in \mathbb{R}
$$

la operación de contracción nos devuelve un tensor de tipo $\left(\begin{array}{l}0 \\ 0\end{array}\right)$,es decir, una función escalar.

\section{Permutación de índices}

Consiste en el cambio de orden de dos o más índices de la expresión del tensor inicial.

El tensor obtenido diferirá del tensor inicial.

Ejemplo:

Dado el tensor $A^{i_{1} i_{2}} j_{1} j_{2}(x)$ de tipo $\left(\begin{array}{l}2 \\ 2\end{array}\right)$ la permutación de índices puede generar otros tensores como por ejemplo

$$
\begin{aligned}
& B_{j_{1} j_{2}}^{i_{1} i_{2}}(x)=A^{i_{2} i_{1}} j_{1} j_{2}(x) \\
& C_{j_{1} j_{2}}^{i_{1} i_{2}}(x)=A^{i_{1} i_{2}} j_{2} j_{1}(x)
\end{aligned}
$$

Derivada de la operación permutación de índices podemos definir las siguientes operaciones:

\section{Simetrización}

Esta operación se realiza respecto a cualesquiera $N$ índices de un mismo tipo de un tensor dado. En el caso de simetrización respecto a dos índices ésta se define como sigue

$$
\begin{aligned}
& A_{i_{1} i_{2} \cdots i_{k}}^{\left(j_{1} j_{2}\right) \cdots j_{l}}(x)=\frac{1}{2 !}\left[A_{i_{1} i_{2} \cdots i_{k}}^{j_{1} j_{2} \cdots j_{l}}(x)+A_{i_{1} i_{2} \cdots i_{k}}^{j_{2} j_{1} \cdots j_{l}}(x)\right] \\
& A_{\left(i_{1} i_{2}\right) \cdots i_{k}}^{j_{1} j_{j} \cdots j_{l}}(x)=\frac{1}{2 !}\left[A_{i_{1} i_{2} \cdots i_{k}}^{j_{1} j_{2} \cdots j_{l}}(x)+A_{i_{2} i_{1} \cdots i_{k}}^{j_{1} j_{2} \cdots j_{l}}(x)\right]
\end{aligned}
$$

Alternización

Esta operación se realiza respecto a cualesquiera $N$ índices de un mismo tipo de un tensor dado. En el caso de alternización respecto a dos índices ésta se define como sigue

$$
A_{i_{1} i_{2} \cdots i_{k}}^{\left[j_{1} j_{2}\right] \cdots j_{l}}(x)=\frac{1}{2 !}\left[A_{i_{1} i_{2} \cdots i_{k}}^{j_{1} j_{2} \cdots j_{l}}(x)-A_{i_{1} i_{2} \cdots i_{k}}^{j_{2} j_{1} \cdots j_{l}}(x)\right]
$$




$$
A_{\left[i_{1} i_{2}\right] \cdots i_{k}}^{j_{1} j_{2} \cdots j_{l}}(x)=\frac{1}{2 !}\left[A_{i_{1} i_{2} \cdots i_{k}}^{j_{1} j_{2} \cdots j_{l}}(x)-A_{i_{2} i_{1} \cdots i_{k}}^{j_{1} j_{2} \cdots j_{l}}(x)\right]
$$

El símbolo de kronecker $\delta_{k}^{i}$ constituye un tensor de componentes

$$
\delta_{k}^{i}=\left\{\begin{array}{lll}
1 & \text { si } & i=k \\
0 & \text { si } & i \neq k
\end{array}\right.
$$

en cualquier sistema coordenado.

\section{Derivada covariante}

Importante: Queremos trabajar intrínsecamente, esto es, que ninguno de las magnitudes que estudiemos hagan referencia a nada que no esté dentro de la propia variedad diferenciable.

Cuando se derivan campos vectoriales (definidos en el espacio tangente a la variedad) el resultado de esta derivación no tiene porqué ser otro campo, ya que, puede que el resultado de dicha derivación no se pueda expresar como combinación lineal de elementos de la base del espacio tangente. Esto se ve claramente cuando se trabaja con superficies regulares (inmersas en $\mathbb{R}^{3}$ ), si entendemos los vectores tangentes como velocidades entonces su derivada convencional (heredada de $\mathbb{R}^{3}$ ), esto es, su aceleración será un vector que se sale del plano tangente, por lo que dicha aceleración no puede ser un campo definido en la variedad.

De ahí que haya que redefinir el concepto de derivada de un campo vectorial. Y a partir de ahí extender dicha derivación al resto del conjunto de entes geométricos, en particular a los tensores.

Podemos intentar derivar un campo de vectores contravariante $\boldsymbol{V}$ cualquiera de componentes $V^{i}$

$$
\begin{gathered}
\frac{\partial \boldsymbol{V}}{\partial x^{\alpha}}=\frac{\partial}{\partial x^{\alpha}}\left(V^{i} \mathbf{e}_{i}\right)=\frac{\partial V^{i}}{\partial x^{\alpha}} \mathbf{e}_{i}+V^{i} \frac{\partial \mathbf{e}_{i}}{\partial x^{\alpha}} \\
\frac{\partial \mathbf{e}_{\alpha}}{\partial x^{\beta}}=\Gamma_{\alpha \beta}^{\mu} \mathbf{e}_{\mu} \\
\frac{\partial \boldsymbol{V}}{\partial x^{\beta}}=\left(\frac{\partial V^{\alpha}}{\partial x^{\beta}}+V^{\mu} \Gamma_{\mu \beta}^{\alpha}\right) \mathbf{e}_{\alpha}
\end{gathered}
$$


Por ejemplo, en coordenadas polares

$$
\begin{aligned}
\Gamma_{r \theta}^{\theta} & =\Gamma_{\theta r}^{\theta}=\frac{1}{r} \\
\Gamma_{\theta \theta}^{\theta} & =0 \\
\Gamma_{r r}^{\theta} & =\Gamma_{r r}^{r}=0 \\
\Gamma_{\theta r}^{r} & =\Gamma_{r \theta}^{r}=0 \\
\Gamma_{\theta \theta}^{r} & =-r
\end{aligned}
$$

esto es

$$
\begin{array}{cc}
\frac{\partial \mathbf{e}_{r}}{\partial r}=0 & \frac{\partial \mathbf{e}_{r}}{\partial \theta}=\frac{1}{r} \mathbf{e}_{\theta} \\
\frac{\partial \mathbf{e}_{\theta}}{\partial r}=\frac{1}{r} \mathbf{e}_{\theta} & \frac{\partial \mathbf{e}_{\theta}}{\partial \theta}=-r \mathbf{e}_{r}
\end{array}
$$

Importante: Los Símbolos de Christoffel posibilitan expresar las derivadas de los vectores de la base sin utilizar ningún otro sistema coordenado. Esto es, nos posibilita hacer los cálculos de forma intrínseca.

La derivada covariante del vector contravariante $V^{i}$ será

$$
\nabla_{l} A^{i}=A_{; l}^{i}=\frac{\partial A^{i}}{\partial x^{l}}+\Gamma_{k l}^{i} A^{k}
$$

podemos construir la derivada covariante de un covector (vector covariante) $B_{i}$ como

$$
\nabla_{l} B_{i}=\frac{\partial B_{i}}{\partial x^{l}}-\Gamma_{i l}^{k} B_{k}
$$

El Teorema Egregio de Gauss muestra cómo toda información de interés geométrico puede expresarse en términos de la métrica, esto es, de la primera forma fundamental, en otras palabras, no necesitamos recurrir a ningún espacio exterior a la variedad para definir nuestros conceptos geométricos.

Así podemos construir una geometría intrínseca a partir de los coeficientes de la conexión o Símbolos de Christoffel

Por tanto no podemos definir la curvatura de una curva, superficie, etc... por la manera en que dicha superficie ocupa el espacio.

Por eso deberemos renunciar por ejemplo al concepto de curvatura media pero no al de curvatura gaussiana

Los símbolos de Christoffel $\Gamma_{k l}^{i}$ (conexiones del espacio de Riemann) se calculan como

$$
\Gamma_{k l}^{i}=\frac{1}{2} g^{i m}\left(\frac{\partial g_{m l}}{\partial x^{k}}+\frac{\partial g_{m k}}{\partial x^{l}}-\frac{\partial g_{k l}}{\partial x^{m}}\right)
$$


Los Símbolos de Christoffel no son tensores, en cambio son entes geométricos lineales no homogéneos de segundo orden, pues

$$
\Gamma_{k l}^{\prime i}\left(x^{\prime}\right)=\Gamma_{m s}^{p}\left(x\left(x^{\prime}\right)\right) \frac{\partial x^{\prime i}}{\partial x^{p}} \frac{\partial x^{m}}{\partial x^{\prime k}} \frac{\partial x^{s}}{\partial x^{\prime l}}+\frac{\partial x^{\prime} i}{\partial x^{p}} \frac{\partial^{2} x^{p}}{\partial x^{\prime k}}
$$

Por ejemplo, en coordenadas polares

$$
g_{r r}=1, g_{\theta \theta}=r^{2}
$$

sustituyendo en B.95 obtenemos los coeficientes no nulos

$$
\begin{aligned}
& \Gamma_{r \theta}^{\theta}=\Gamma_{\theta r}^{\theta}=\frac{1}{r} \\
& \Gamma_{\theta \theta}^{r}=-r
\end{aligned}
$$

Aquí se ve claro que los símbolos de Christoffel no son componentes de un tensor, ya que en el espacio euclídeo en coordenadas cartesianas sabemos que $\Gamma_{j k}^{i}$ se anulan ideénticamente y en coordenadas polares no es así.

Consideremos una superficie regular $M$, esto es, una variedad diferenciable inmersa en $\mathbb{R}^{3}$

Si tomamos un campo $Y(x)$ tangente a $M$ y lo restringimos a la curva parametrizada $\alpha(\tau) \in M$, podremos asociar a este campo otro en $\mathbb{R}^{3}$ donde estudiar su variación a lo largo de la curva $\alpha(\tau)$ a través de la derivada convencional $\frac{d Y}{d t} \in \mathbb{R}^{3}$. Como este nuevo campo puede tener componentes que hagan que los vectores asociados a cada punto de la curva se salgan de la propia superficie nos quedaremo únicamente con la parte tangencial a esta aplicando una proyección que denotaremos por $\frac{\nabla Y}{d t}$ que llamaremos derivada intrínseca.

Así si tomamos una curva $\alpha(\tau) \in M$ tal que $\alpha(0)=p$ y $\dot{\boldsymbol{\alpha}}(0)=\boldsymbol{U}$, podemos definir derivada covariante $\nabla_{U} Y$ de un campo vectorial $Y$ en la dirección del vector $U$ como la derivada intrínseca $\nabla_{U} Y:=\frac{\nabla Y}{d t}$.

El desplazamiento paralelo de un vector $Y$ a lo largo de la curva se define como $\nabla_{U} Y=0$.

Sean los campos vectoriales $X$ e $Y$ definidos en un entorno de un punto $p$ de la variedad diferenciable $M$ y los vectores $U$ y $V$ definidos en $p \in M$. Definimos derivada covariante como un operador $\nabla$ en $p$, tal que a cada $X$ y $U$ le asigna un vector $\nabla_{U} X$ y cumplen las siguientes propiedades 


$$
\begin{aligned}
\nabla_{U}(a X+b Y) & =a \nabla_{U} X+b \nabla_{U} Y \\
\nabla_{a U+b V} X & =a \nabla_{U} X+b \nabla_{V} X \\
\nabla_{U}(f(x) X) & =f(x) \nabla_{U} X+(U f) X
\end{aligned}
$$

con $f(x)$ función diferenciable, y $a, b \in \mathbb{R}$ además $U(f)=d f(U)$

Tomemos los vectores de la base del espacio tangente en el punto $p\left\{\frac{\partial}{\partial x^{\alpha}}\right\}$, así los campos $X$ e $Y$ pueden expresarse como $X=X^{i} \frac{\partial}{\partial x^{i}}$ e $Y=Y^{j} \frac{\partial}{\partial x^{j}}$ y aplicando las propiedades anteriores obtenemos

$$
\begin{aligned}
\nabla_{X} Y=\nabla_{X^{i} \frac{\partial}{\partial x^{i}}}\left(Y^{j} \frac{\partial}{\partial x^{j}}\right) & =X^{i} \nabla_{\frac{\partial}{\partial x^{i}}}\left(Y^{j} \frac{\partial}{\partial x^{j}}\right)=\left(X^{i} \partial_{x^{i}} Y^{j}\right) \partial_{x^{j}}+X^{i} Y^{j} \nabla_{\frac{\partial}{\partial x^{i}}} \frac{\partial}{\partial x^{j}} \\
\nabla_{\frac{\partial}{\partial x^{i}}} \frac{\partial}{\partial x^{j}} & =\Gamma_{i j}^{k} \partial_{x}^{k} \quad \text { Simbolos de Christoffel }
\end{aligned}
$$

por tanto, las componentes del campo $\nabla_{X} Y$ serán

$$
\left(\nabla_{X} Y\right)^{i}=X^{j} \frac{\partial Y^{i}}{\partial x^{j}}+\left(\Gamma_{j k}^{i} X^{j} Y^{k}\right) X^{j}
$$

podemos definir la conexión como la 1-forma

$$
\nabla Y^{i}=d Y^{i}+\Gamma_{j k}^{i} Y^{k} d x^{j}
$$

La operación $\nabla_{X} Y$ también se denomina conexión afín.

En una variedad podemos definir un sistema coordenado afín como $n$ campos vectoriales $\mathbf{e}_{\alpha}=\frac{\partial}{\partial x^{\alpha}}(\alpha=1,2, \cdots, n)$ linealmente independientes que forman una base del espacio tangente en cada punto. Así los Símbolos de Christoffel serán los coeficientes de la conexión afín.

Sean los campos vectoriales $X, Y$ y $Z$, existe un única conexión $\nabla$ en una variedad Riemanniana $M$ llamada conexión de Levi-Civita que cumple ser libre de torsión

$$
\nabla_{X} Y-\nabla_{Y} X=[X, Y]
$$

y compatible con la métrica

$$
X<Y, Z>=<\nabla_{X} Y, Z>+<Y, \nabla_{X} Z>
$$

La torsión libre requiere $\Gamma_{j k}^{i}=\Gamma_{k j}^{i}$

\section{Derivada covariante a lo largo de una curva}


Sea una curva $\alpha(\tau)$ definida sobre la variedad $M$ y pasando por el punto $p$ con vector tangente $\dot{\alpha}(\tau)=\frac{d \alpha}{d \tau}=T$ y sea un campo vectorial $X$ definido sobre la curva $\alpha(\tau)$, dada una carta la derivada covariante $\nabla_{T} X$ puede escribirse como

$$
\nabla_{T} X=\frac{\nabla X}{d \tau} \equiv \frac{D X}{d \tau}=\left[\frac{d}{d t} X^{i}+\Gamma_{j k}^{i} \frac{d x^{j}}{d \tau} Y^{j}\right] \frac{\partial}{\partial x^{i}}
$$

Podemos definir el transporte paralelo de un vector $Y$ a lo largo de una curva $\alpha(\tau)$ si se cumple

$$
\frac{\nabla Y}{d \tau} \equiv \frac{D Y}{d \tau}=\nabla_{T} Y=0
$$

Si dos vectores $U, V$ son trasladados paralelamente a lo largo de la curva $\alpha(\tau)$, se cumple

$$
<X, Y>=\text { constante }
$$

siendo $X$ e $Y$ los trasladados paralelamente de los vectores $U$ y $V$. 


\section{Apéndice $\mathrm{C}$}

\section{Principio de mínima curvatura de Hertz}

El Principio de mínima curvatura de Hertz afirma que todo sistema dinámico evolucionará siguiendo la trayectoria de mínima curvatura.

Dada la identificación entre trayectorias físicas y geodésicas de la variedad mecánica, demostraremos este principio utilizando la métrica Jacobi.

Consideramos que nos movemos a lo largo de una trayectoria del sistema, esto es, sobre una geodésica de nuestra variedad mecánica, de tal manera que el vector $\mathbf{w}$ se corresponderá con el tangente a esta curva, esto es, $\mathbf{w}=\dot{\gamma}$.

Definimos la aplicación autoadjunta $\mathcal{A}$ como

$$
\begin{aligned}
\mathcal{A}: & \mathbb{V} \longrightarrow \mathbb{V} \\
& \mathbf{v} \longmapsto R(\mathbf{w}, \mathbf{v}) \mathbf{w}=R(\dot{\gamma}, \mathbf{v}) \dot{\gamma} .
\end{aligned}
$$

Buscamos la matriz asociada a esta aplicación lineal. Para ello, en dimensión dos, utilizaremos una base cualquiera del espacio vectorial $\left\{\mathbf{e}_{1}, \mathbf{e}_{2}\right\}$. Entonces

$$
\begin{aligned}
& \dot{\gamma}=\dot{\gamma}^{i} \mathbf{e}_{i} \equiv \dot{\gamma}^{1} \mathbf{e}_{1}+\dot{\gamma}^{2} \mathbf{e}_{2}, \\
& \mathbf{v}=v^{i} \mathbf{e}_{i} \equiv v^{1} \mathbf{e}_{1}+v^{2} \mathbf{e}_{2} .
\end{aligned}
$$

Entonces, teniendo en cuenta que

$$
\begin{gathered}
R\left(\mathbf{e}_{i}, \mathbf{e}_{j}\right) \mathbf{e}_{k}=R_{i j k}^{l} \mathbf{e}_{l}, \\
R(\dot{\gamma}, \mathbf{v}) \dot{\gamma}=-v^{i} \dot{\gamma}^{l} \dot{\gamma}^{k} R_{l i k}^{m} \mathbf{e}_{m} .
\end{gathered}
$$

Asociaremos a la aplicación autoadjunta A la matriz $K_{i j}$ aplicando los elementos de la base $\left\{\mathbf{e}_{i}\right\}$ y proyectando la imagen sobre estos mismos vectores de la base, esto es

$$
\left.K_{i j}=<R\left(\dot{\gamma}, \mathbf{e}_{i}\right) \dot{\gamma}, \mathbf{e}_{j}\right)>\Rightarrow
$$




$$
\begin{aligned}
& \left.K_{11}=<R\left(\dot{\gamma}, \mathbf{e}_{1}\right) \dot{\gamma}, \mathbf{e}_{1}\right)>=<\left(\dot{\gamma}^{1}\right)^{2} R_{212}^{m} \mathbf{e}_{m}, \mathbf{e}_{1}>=\left(\dot{\gamma}^{2}\right)^{2} g_{m 1} R_{212}^{m}=\left(\dot{\gamma}^{2}\right)^{2} R_{1212} \\
& \left.K_{12}=<R\left(\dot{\gamma}, \mathbf{e}_{1}\right) \dot{\gamma}, \mathbf{e}_{2}\right)>=<\dot{\gamma}^{2} \dot{\gamma}^{1} R_{211}^{m} \mathbf{e}_{m}, \mathbf{e}_{2}>=\dot{\gamma}^{2} \dot{\gamma}^{1} g_{m 2} R_{211}^{m}=\dot{\gamma}^{2} \dot{\gamma}^{1} R_{2112}= \\
& -\dot{\gamma}^{2} \dot{\gamma}^{1} R_{1212} \\
& \left.K_{21}=<R\left(\dot{\gamma}, \mathbf{e}_{2}\right) \dot{\gamma}, \mathbf{e}_{1}\right)>=<\dot{\gamma}^{1} \dot{\gamma}^{2} R_{212}^{m} \mathbf{e}_{m}, \mathbf{e}_{1}>=\dot{\gamma}^{2} \dot{\gamma}^{1} g_{m 1} R_{212}^{m}=\dot{\gamma}^{2} \dot{\gamma}^{1} R_{2112}= \\
& -\dot{\gamma}^{2} \dot{\gamma}^{1} R_{1212} \\
& \left.K_{22}=<R\left(\dot{\gamma}, \mathbf{e}_{2}\right) \dot{\gamma}, \mathbf{e}_{2}\right)>=<\left(\dot{\gamma}^{1}\right)^{2} R_{212}^{m} \mathbf{e}_{m}, \mathbf{e}_{1}>=\left(\dot{\gamma}^{1}\right)^{2} g_{m 2} R_{211}^{m}=\left(\dot{\gamma}^{1}\right)^{2} R_{1212}
\end{aligned}
$$

$$
K_{a b}=\left(\begin{array}{cc}
\left(\dot{\gamma}^{2}\right)^{2} R_{1212} & -\dot{\gamma}^{2} \dot{\gamma}^{1} R_{1212} \\
-\dot{\gamma}^{2} \dot{\gamma}^{1} R_{1212} & \left(\dot{\gamma}^{1}\right)^{2} R_{1212}
\end{array}\right)
$$

por tanto la matriz $K_{a b}$ es simétrica y la aplicación lineal asociada es autoadjunta, ésta puede expresarse tensorialmente como

$$
\mathcal{A}=K_{i j} \mathbf{e}^{i} \otimes \mathbf{e}^{j} .
$$

Ahora bien, la matriz A representa una aplicación $\mathbb{V} \longrightarrow \mathbb{V}^{*}$ por lo que para obtener una aplicación $\mathbb{V} \longrightarrow \mathbb{V}$ tendremos que subirle un índice a las componentes de este tensor utilizando la métrica $g_{i j}$. Entonces

$$
K_{j}^{i}=K_{i l} g^{l j} .
$$

De esta forma

$$
\mathcal{A}=R(\dot{\gamma}, \bullet) \dot{\gamma}=\underbrace{\dot{\gamma}^{l} \dot{\gamma}^{m} R_{l i m}^{k}}_{\mathcal{K}_{i}^{k}} \mathbf{e}^{i} \otimes \mathbf{e}_{k},
$$

donde $\mathcal{K}_{i}^{k} \equiv$ tensor de estabilidad será

$$
\mathcal{K}_{b}^{a}=\left(\begin{array}{cc}
\left(\dot{\gamma}^{2}\right)^{2} R_{212}^{1} & -\dot{\gamma}^{2} \dot{\gamma}^{1} R_{212}^{1} \\
-\dot{\gamma}^{2} \dot{\gamma}^{1} R_{212}^{1} & \left(\dot{\gamma}^{1}\right)^{2} R_{212}^{1}
\end{array}\right)
$$

Diagonalizando la matriz obtenemos el polinomio característico

$$
\left|\begin{array}{cc}
\left(\dot{\gamma}^{2}\right)^{2}-\lambda & -\dot{\gamma}^{2} \dot{\gamma}^{1} \\
-\dot{\gamma}^{2} \dot{\gamma}^{1} & \left(\dot{\gamma}^{1}\right)^{2}-\lambda
\end{array}\right|=0 \Rightarrow \lambda\left(\lambda-\left(\left(\dot{\gamma}^{1}\right)^{2}+\left(\dot{\gamma}^{2}\right)^{2}\right)\right)=0
$$

cuyos autovalores serán $\lambda_{1}=0$ y $\lambda_{2}=R_{212}^{1}\left(\left(\dot{\gamma}^{1}\right)^{2}+\left(\dot{\gamma}^{2}\right)^{2}\right)$.

Se deduce que $\mathcal{A}\left(\mathbf{e}_{(1)}\right)=\lambda_{1} \mathbf{e}_{(1)}=0$, por lo que

$\lambda_{1}=\operatorname{mín}<\mathcal{A}\left(\mathbf{e}_{(1)}\right), \mathbf{e}_{(1)}>=\operatorname{mín}<R\left(\dot{\gamma}, \mathbf{e}_{(1)}\right) \dot{\gamma}, \mathbf{e}_{(1)}>$

Por tanto, tomando $\mathbf{e}_{(1)}$ como el vector tangente a la trayectoria, obtendremos la dirección de mínima curvatura de la variedad mecánica.

Geométricamente, los autovectores serán: 
- Para $\lambda_{1}=0$ el autovector correspondiente $\mathbf{v}=v^{i} \mathbf{e}_{i}$ cumplirá $\dot{\gamma}^{2} v^{1}-$ $\dot{\gamma}^{1} v^{2}=0$, por lo que con métrica euclídea $\left\langle\dot{\gamma}^{\perp}, \mathbf{v}\right\rangle=0$ de forma que $\dot{\gamma} / / \mathbf{v}$.

- Para $\lambda_{2}=R_{212}^{1}\left(\left(\dot{\gamma}^{1}\right)^{2}+\left(\dot{\gamma}^{2}\right)^{2}\right)$ el autovector correspondiente cumplirá $\dot{\gamma}^{1} v^{1}+\dot{\gamma}^{2} v^{2}=0$, por lo que, $\langle\dot{\gamma}, \mathbf{v}>=0$ de manera que, con métrica euclídea, deben cun como $\lambda_{1}=\underbrace{\text { mín. }}_{\|\mathbf{u}\|=1}<\mathcal{A}(\mathbf{u}), \mathbf{u}>\quad$ y $\quad \lambda_{2}=\underbrace{\operatorname{máx}}_{\|\mathbf{u}\|=1}<\mathcal{A}(\mathbf{u}), \mathbf{u}>$

De lo anterior se concluye que la dirección tangente a la trayectoria es la de mínima curvatura. 



\section{Apéndice D}

\section{Teorema de Gauss-Bonnet}

Consideramos el Teorema de Gauss-Bonnet local de geometría diferencial, que dice

$$
\sum_{i=1}^{p} \int_{C_{i}} k_{g}(s) d s+\iint_{R} \frac{\mathcal{R}}{2} d \sigma+\sum_{i=1}^{p} \theta_{i}=2 \pi \chi(R)
$$

con $\mathcal{R} \equiv$ curvatura gaussiana de la variedad riemanniana $k_{g} \equiv$ curvatura geodésica de las curvas frontera de la región $R$ $\chi(R)=$ característica de Euler-Poincaré de la región $R(\in \mathbb{Z})$ $d \sigma=$ diferencial de Área $\left(d \sigma=|g| d x^{1} d x^{2} \ldots d x^{n}\right)$

$\theta_{i} \equiv$ ángulos formados por curvas frontera de la región $R$

Si la curva es una trayectoria del sistema dinámico entonces será geodésica de la variedad mecánica, por tanto, $K_{g}(s)=0$, si además es cerrada $\theta_{i}=0$, entonces para toda trayectoria cerrada, se cumple

$$
\iint_{R} \frac{\mathcal{R}}{2} d \sigma=2 \pi \chi(R)
$$

